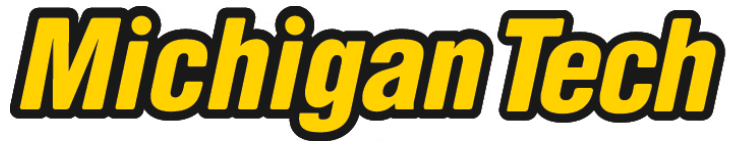 \\ Michigan Technological University Create the Future Digital Commons @ Michigan Tech
}

2013

\section{AMPLITUDE-VERSUS-ANGLE ANALYSIS AND WIDE-ANGLE- INVERSION OF CROSSWELL SEISMIC DATA IN A CARBONATE RESERVOIR}

Mohamed S. Ibrahim

Michigan Technological University

Follow this and additional works at: https://digitalcommons.mtu.edu/etds

Part of the Geophysics and Seismology Commons

Copyright 2013 Mohamed S. Ibrahim

Recommended Citation

Ibrahim, Mohamed S., "AMPLITUDE-VERSUS-ANGLE ANALYSIS AND WIDE-ANGLE-INVERSION OF CROSSWELL SEISMIC DATA IN A CARBONATE RESERVOIR", Dissertation, Michigan Technological University, 2013.

https://doi.org/10.37099/mtu.dc.etds/627

Follow this and additional works at: https://digitalcommons.mtu.edu/etds

Part of the Geophysics and Seismology Commons 


\section{AMPLITUDE-VERSUS-ANGLE ANALYSIS AND WIDE-ANGLE-INVERSION OF CROSSWELL SEISMIC DATA IN A CARBONATE RESERVOIR}

\section{By}

Mohamed S. Ibrahim

\section{A DISSERTATION}

Submitted in partial fulfillment of the requirements for the degree of DOCTOR OF PHILOSOPHY

In Geophysics

MICHIGAN TECHNOLOGICAL UNIVERSITY 
This dissertation has been approved in partial fulfillment of the requirements for the Degree of DOCTOR OF PHILOSOPHY in Geophysics.

Department of Geological and Mining Engineering and Sciences

Dissertation Advisor: Dr. Wayne D. Pennington

Committee Member: Dr. Roger M. Turpening

Committee Member: $\quad$ Dr. Gregory P. Waite

Committee Member: Dr. Tamara R. Olson

Department Chair: Dr. Wayne D Pennington 


\section{Table of Contents}

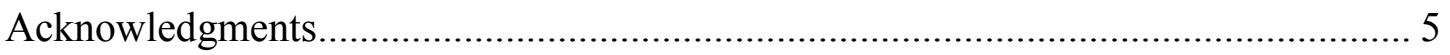

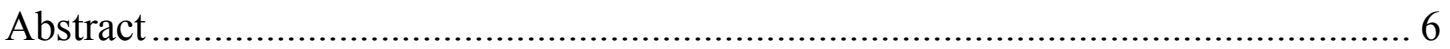

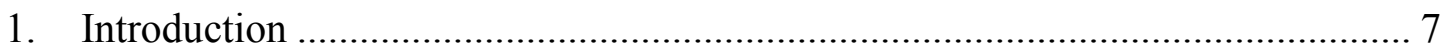

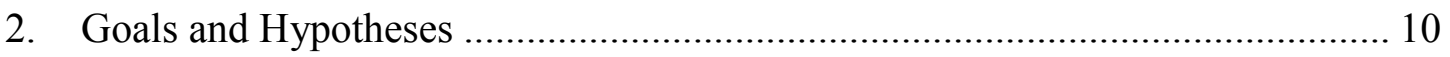

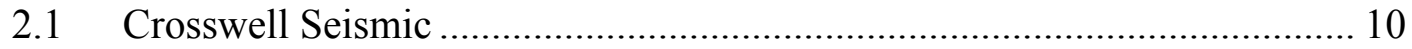

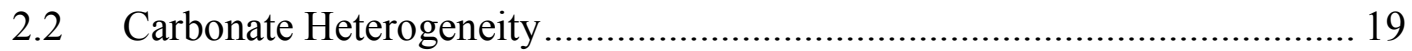

2.3 Reflections from above versus from beneath ......................................... 22

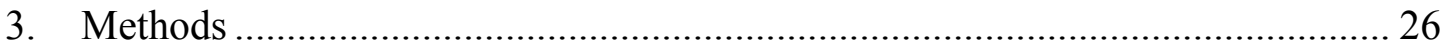

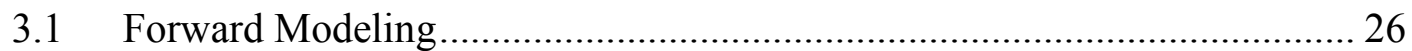

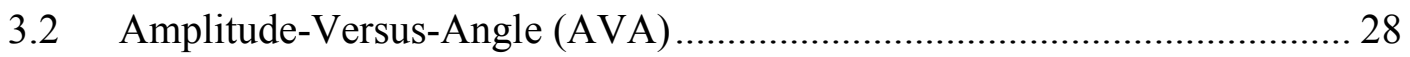

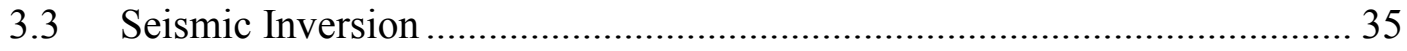

4. Crosswell Seismic Imaging of Acoustic and Shear Impedance in a Michigan

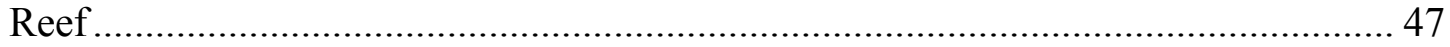

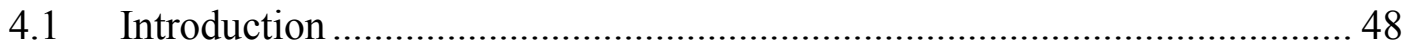

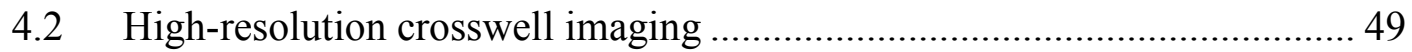

4.3 "From above" versus "from beneath" CSSI inversion................................ 54

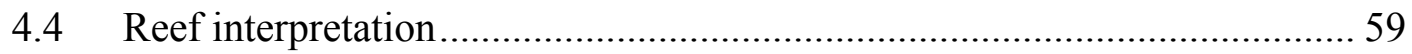

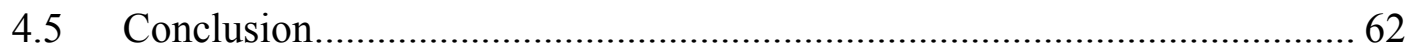

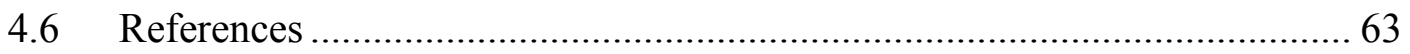

5. AVA Analysis and Inversion of Crosswell Seismic Images taken from Above

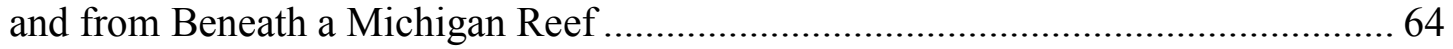

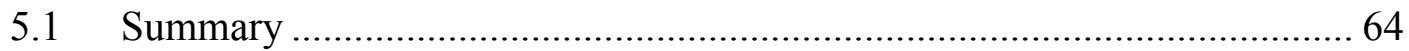

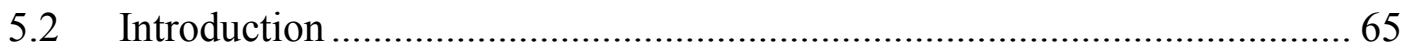




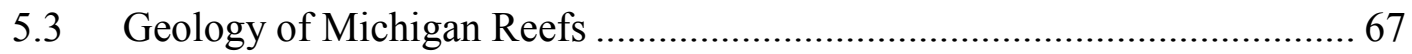

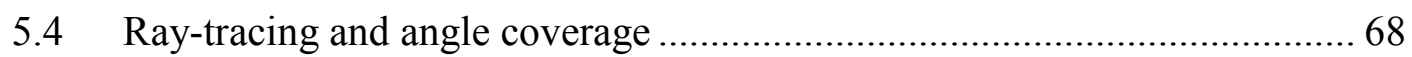

5.5 AVA analysis and ideal Zoeppritz equations .......................................... 72

5.6 Combined model for inversion from above and from beneath ................. 80

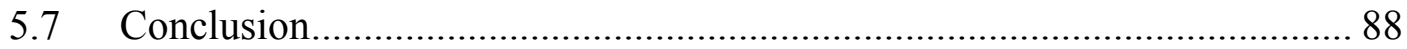

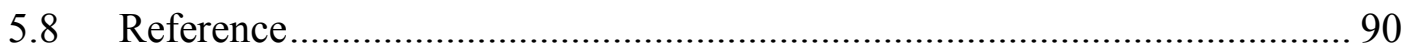

6. Geostatistical Inversion of Crosswell Seismic data over Michigan Reefs .......... 94

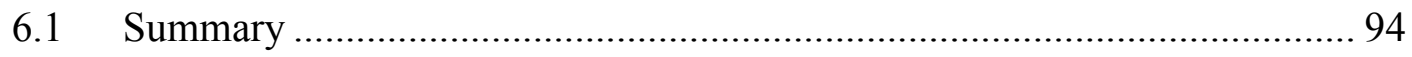

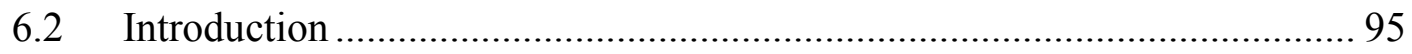

6.3 Deterministic and Geostatisitical inversion at Coldspring ....................... 99

6.4 Geostatistical inversion at Springdale ................................................ 108

6.5 Conclusion.................................................................................... 117

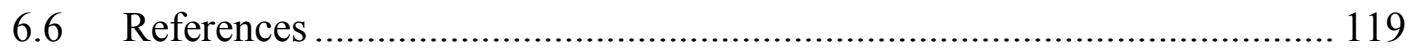

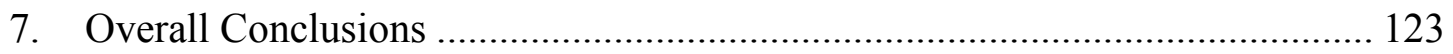

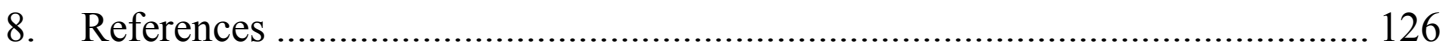




\section{Acknowledgments}

First of all, I am grateful to The Almighty Allah for helping me to complete this dissertation.

I wish to express my sincere thanks and grateful to my principle supervisor, Dr. Wayne Pennington. This dissertation would not have been possible without his help, support and patience, not to mention his advice and abundant knowledge in seismic petrophysics.

I wish to express my sincere thanks for my second supervisor, Dr. Roger Turpening for his great advice, support and invaluable discussions. Also, I would like to appreciate his 'warm' hospitality during the SPOT lab meetings!

Also, I would like to thank the dissertation committee: Dr. Tamara Olson and Dr. Gregory Waite for their encouragement and insightful comments.

Many thanks to the US Department of Energy, National Energy Technology Laboratory (Tulsa) for supporting this work under contract DE-FC26-04NT15508.

We are grateful to Z-Seis, Inc. (now Schlumberger DeepLook-CS), for acquisition and processing of the crosswell seismic data.

Special gratitude goes to Jason (a CGG company) for their technical and software support.

Also, special gratitude goes Merit Energy for providing data for the producing well in the reef.

Above all, I would like to thank my wife Eman for her personal support and great patience at all times. My father, mother and parents-in-low have given me their complete support throughout, as always, for which my mere expression of thanks likewise does not suffice. 


\section{Abstract}

Crosswell data set contains a range of angles limited only by the geometry of the source and receiver configuration, the separation of the boreholes and the depth to the target. However, the wide angles reflections present in crosswell imaging result in amplitude-versus-angle (AVA) features not usually observed in surface data. These features include reflections from angles that are near critical and beyond critical for many of the interfaces; some of these reflections are visible only for a small range of angles, presumably near their critical angle.

High-resolution crosswell seismic surveys were conducted over a Silurian (Niagaran) reef at two fields in northern Michigan, Springdale and Coldspring. The Springdale wells extended to much greater depths than the reef, and imaging was conducted from above and from beneath the reef. Combining the results from images obtained from above with those from beneath provides additional information, by exhibiting ranges of angles that are different for the two images, especially for reflectors at shallow depths, and second, by providing additional constraints on the solutions for Zoeppritz equations.

Inversion of seismic data for impedance has become a standard part of the workflow for quantitative reservoir characterization. Inversion of crosswell data using either deterministic or geostatistical methods can lead to poor results with phase change beyond the critical angle, however, the simultaneous pre-stack inversion of partial angle stacks may be best conducted with restrictions to angles less than critical.

Deterministic inversion is designed to yield only a single model of elastic properties (best-fit), while the geostatistical inversion produces multiple models (realizations) of elastic properties, lithology and reservoir properties. Geostatistical inversion produces results with far more detail than deterministic inversion. The magnitude of difference in details between both types of inversion becomes increasingly pronounced for thinner reservoirs, particularly those beyond the vertical resolution of the seismic.

For any interface imaged from above and from beneath, the results AVA characters must result from identical contrasts in elastic properties in the two sets of images, albeit in reverse order. An inversion approach to handle both datasets simultaneously, at pre-critical angles, is demonstrated in this work.

The main exploration problem for carbonate reefs is determining the porosity distribution. Images of elastic properties, obtained from deterministic and geostatistical simultaneous inversion of a high-resolution crosswell seismic survey were used to obtain the internal structure and reservoir properties (porosity) of Niagaran Michigan reef. The images obtained are the best of any Niagaran pinnacle reef to date. 


\section{Introduction}

Surface-seismic methods have led to a large set of technologies and observations that provide robust evaluation of reservoir properties, but this technology is limited in resolution by frequency contents.

The details of the interior of oil and gas reservoirs are often unknown. For instance, the detection of reefs from surface seismic data is usually restricted to the presence of the reef alone, and internal architecture of that reef remains invisible to the seismic interpreter. In most reservoirs, the internal heterogeneity controls their productivity, and if that heterogeneity is not well-characterized, the reservoir engineer is unable to produce the field optimally.

Crosswell seismic data can be used to fill the gap in resolution and significantly improve the imaging of internal reservoirs heterogeneity. This includes both vertical and lateral heterogeneity of carbonate reservoirs of interest in this study (e.g., Lazaratos, et al., 1991; Lazaratos, 1993; Rector et al., 1995; Ibrahim, et al., 2009, 2010) due to the high signal frequencies and the closer location of sources and receivers to the reflectors. But the wide ranges of angles present in crosswell seismic data have not generally been exploited in quantitative analysis for elastic properties.

This dissertation demonstrates the advantage of crosswell imaging and the information contained in its wide-angle reflections. These reflections should provide additional information beyond that available in typical surface seismic acquisition; in crosswell imaging, this range often includes the critical angle. In many cases, a small acoustic-impedance contrast may mean that the only reflections observed are those from near-critical angles, and inclusion of these angles in a final stacked image can be beneficial (Trisch, 2006; Pennington et al., 2008; Ibrahim et al., 2010). Beyond the critical angle, severe phase rotation of the wavelet occurs, and the stack can be degraded; therefore, for most detailed amplitude variation with angle (AVA) studies, a restriction to narrow range of angles may be prudent (Ibrahim et al., 2009, 2010).

Springdale (Manistee County) and Coldspring (Kalkaska County) are two fields, in northern Michigan, where the crosswell seismic data were acquired and processed in 2005-2006 over pinnacle reefs for the purpose of this project. Both reservoirs have been produced for many years, and although they originally contained exclusively oil (and connate water), they are now also strongly gas-saturated, and produce gas and water in addition to oil. Both reservoirs are essentially volumetric drive, with minimal water encroachment, if any. Thus, they have been drawn down to very low pressures: $25-50 \mathrm{psi}(172-345 \mathrm{kPa})$ at Springdale, and about $1000 \mathrm{psia}(7000 \mathrm{kPa})$ at Coldspring. 
At these two fields, in essence, we conducted three tests. The Springdale site included source and receiver wells outside of the reef. These wells penetrated to depths much greater than the reef depth, so two experiments were conducted at once: imaging "from above" in which the seismic source and receiver locations were above the reflectors, and imaging "from beneath" with tool locations beneath the reflectors. The Coldspring site included source and receiver wells within the reef, providing much greater control over the seismic ties to well logs, in addition to gaining closer access to the target formation for imaging. Imaging the crosswell data in this site was only form above (no imaging was acquired from beneath).

The most valuable aspect of the reservoir characterization in this study has been the inversion of data. Inversion is the process that converts the reflected amplitudes of seismic data into volumes of rock properties (P-impedance, S-impedance or $\mathrm{Vp} / \mathrm{Vs}$ and Density). These properties provide a significant assistance in reservoirs interpretation either quantitatively or qualitatively. Upon the objectives and the data available, inversion can be run using pre- or post-stack seismic data. The inversion workflows that common for oil and gas industry are running using deterministic or geostatiscial approaches.

This dissertation contains the introduction and 5 chapters. The second chapter is the goals and the hypotheses of this work. We will discusse the advantages and pitfalls of using crosswell seismic data over the conventional seismic surveys. Also, we will discuss the carbonate heterogeneity and the geological reef models. The crosswell data from above and the novality importance of the data from beneath will be introduced. In the third chapter, we are going to discusse the methods used to achieve this work. Ray tracing, Amplitude-Versus-Angle and two types of inversion algorithm; deterministic and geostatistical methods are discussed. Also, the difference between pre- and post-stack inversion is included.

In chapter four, we will show our initial effort to invert the crosswell data of Springdale field to generate acoustic and shear impedances. We compare the inversion results using full stack with the partial stacks inversion. Also, the inversion results of the data from beneath and the reason of inverting it individually will be discussed. The procedures to achieve inversion results for data from beneath using the packaged inversion will be discussed as well. At the end of this chapter we introduce an interpretation for the inversion results based on the geological models.

In chapter 5, we extend the inversion approach to invert both the data from above and from beneath combined. Also, we will discuss the importance of full Zoeppritz solution (not the approximation) to crosswell seismic. We will use the seismic gathers of Springdale data in detailed investigation of the amplitude variations with angle of incidence, identifying indicators of contrasts of physical properties at very wide angles from above and from beneath. At the end of this chapter, the benefits of 
acquiring data from beneath to the reservoir characterization studies will be discussed as well.

In chapter 6, we will show how the crosswell seismic inversion is a suitable method of directly measuring interwell changes of reservoir facies and how the variations in elastic properties can be related to reservoir properties; porosity. The data from the two fields; Coldspring and Springdale are used. Also, we will show the differences between the deterministic and gestatisitical inversion in terms of details and uncertainty. Studying the possibility of using geostatisitics in analogous reservoirs is discussed in this chapter as well. The results of ten realizations of elastic properties, lithology probability of porous carbonate and the porosity distribution for both fields are generated, interpreted and the uncertainty was assessed.

Finally, the crosswell seismic images obtained, and interpretations of them, as assisted by inversion approaches and Amplitude-versus-Angle analysis, provide additional insight into the internal geometry of these two reefs and provide data that should be useful for reservoir management. 


\section{Goals and Hypotheses}

\subsection{Crosswell Seismic}

Crosswell seismic data are acquired by placing seismic sources and receivers inside two wellbores close to zone of interest. Two benefits of this type of positioning; first is avoiding signal propagation through attenuative near-surface layers (e.g., glacial till or weathered soils) and the second is, by placing the source and receivers close to the zone of interest, one can obtain a better image for the reservoir zone not possible by the conventional seismic survey.

In crosswell seismic, two types of information are acquired and processed: first, the direct arrivals between source and receivers, and second, reflection information from horizons above and below the source and receiver positions. In addition, there are many other modes and attributes of the crosswell seismic data field that can be captured and processed to yield specific information about the subsurface, such as guided waves and converted waves.

Crosswell seismic surveys were conducted over two sites in Michigan to accomplish two tasks: (1) imaging the carbonate reservoir (Niagaran reefs) between two monitor boreholes in crisp detail; and (2) providing as wide a range as possible of angles of incidence for reflections of seismic waves from within the reef and at the reef's upper surface. The surveys were designed to collect transmitted waves in order to obtain a detailed velocity field through transmission tomography, and to collect reflected waves for high resolution imaging and AVA studies.

The crosswell data in Springdale field, were collected for the main survey using source and receiver locations, every $10 \mathrm{ft}$, from about $2600 \mathrm{ft}$ depth to about $6000 \mathrm{ft}$ depth (figure 2.1). A $0.35 \mathrm{~s}$ sweep from $100 \mathrm{~Hz}$ to $3000 \mathrm{~Hz}$ was stacked 8 times at each depth. This geometry yielded coverage of the reef zone with reflection fold of over 200 and angles of incidence ranging from about $30^{\circ}$ to near $85^{\circ}$. A summary figure of data acquisition is shown in Figure 2.2, providing additional details.

The source was suspended in the Burch well, while the receivers were in the Stech well. The Burch well is cased only down to $2944 \mathrm{ft}$, and is open below that. For each "fan" of shooting, the receiver string is held at a constant depth (using 10 receiver groups at 10-ft spacing for a total span of $100 \mathrm{ft}$ ) while the source is moved up the borehole, firing as it is moved. 8 shots, all within a 10-ft interval, are stacked for each source point. When the source has reached its shallowest point, it is returned to depth, and the receiver string repositioned for the next "fan" of shooting. 


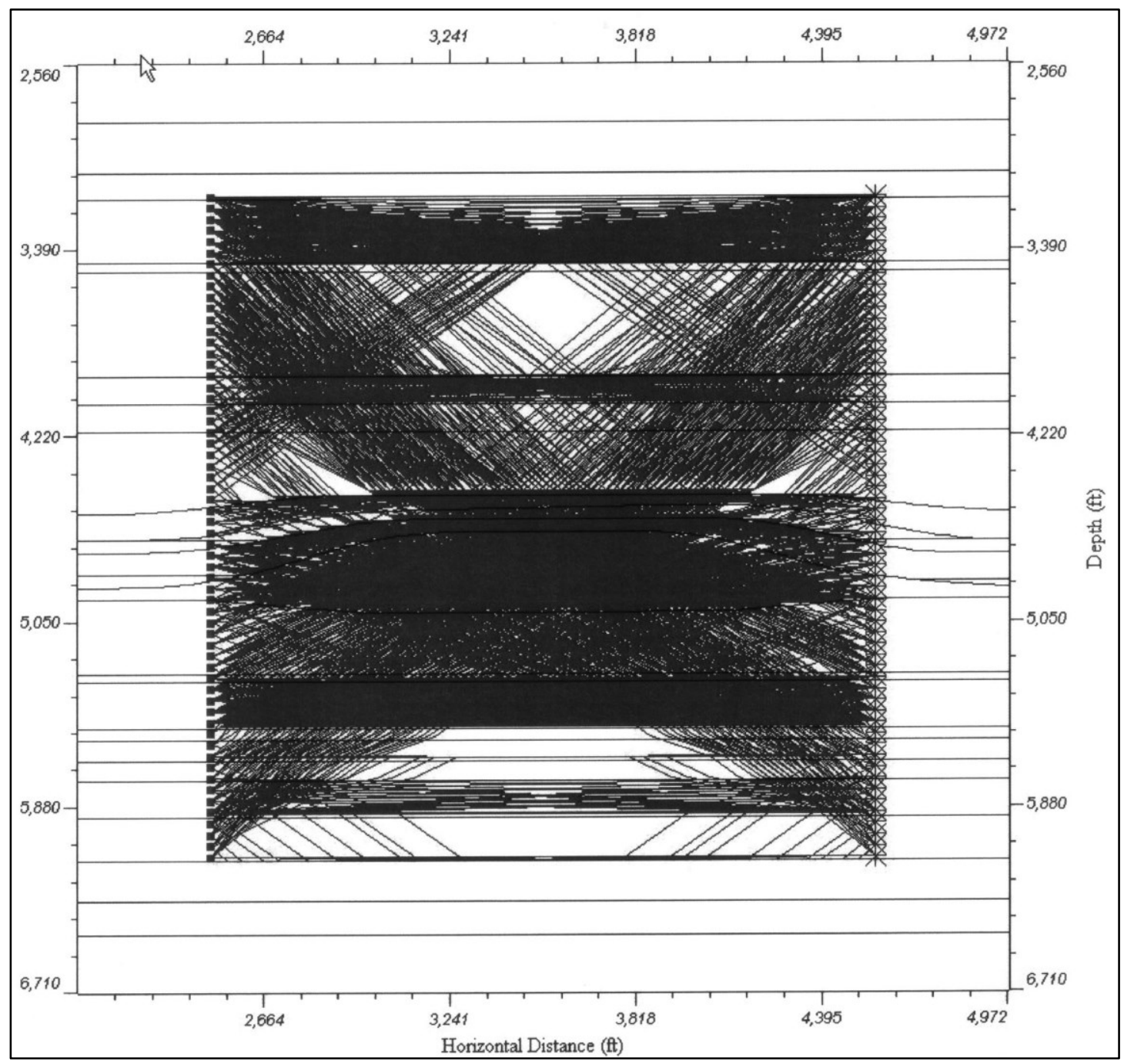

Figure 2.1: Main survey geometry in Springdale field, using (for visualization purposes only) a source and receiver spacing of $50 \mathrm{ft}$. The top and base of the reef is $4650 \mathrm{ft}$ and $4950 \mathrm{ft}$, respectively. This figure is not 1:1 scale, although nearly so. This figure demonstrates that the coverage over the reef and overlying beds are excellent.

One example of a common-receiver gather is shown in Figure 2.3, with a power spectrum for the entire gather.

During the survey, the data contained a typical form of crosswell source-generated noise; tube-wave. This tube-wave noise appeared to be caused by electromagnetic propagation of the source pulse through the earth and conversion to seismic energy at or near the receiver well. The tube-wave noise was easily removed from the data by standard array techniques such as median filtering. 


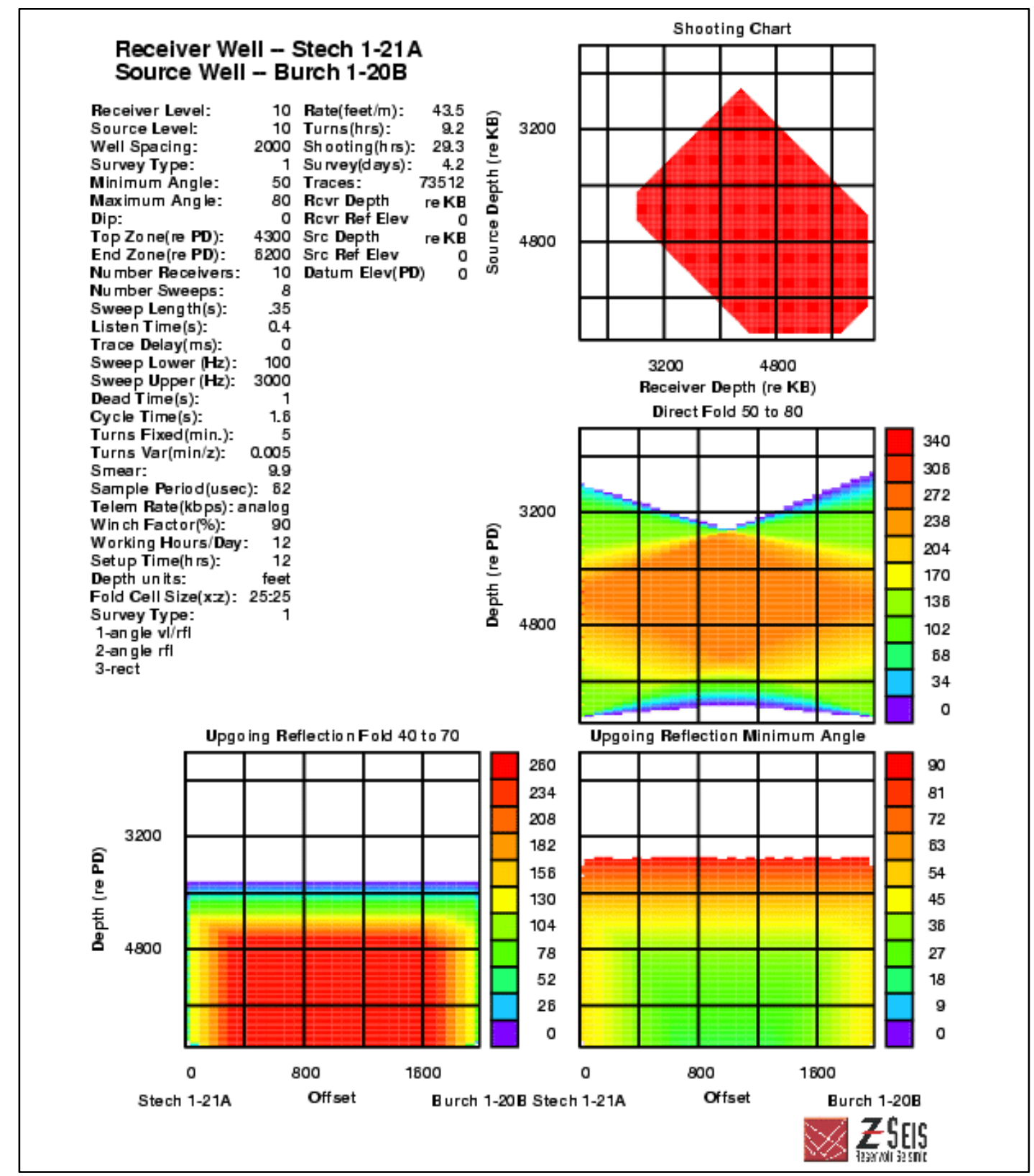

Figure 2.2: Summary of data acquisition in Springdale field (courtesy Deep Look CS previously, Z-Seis) 


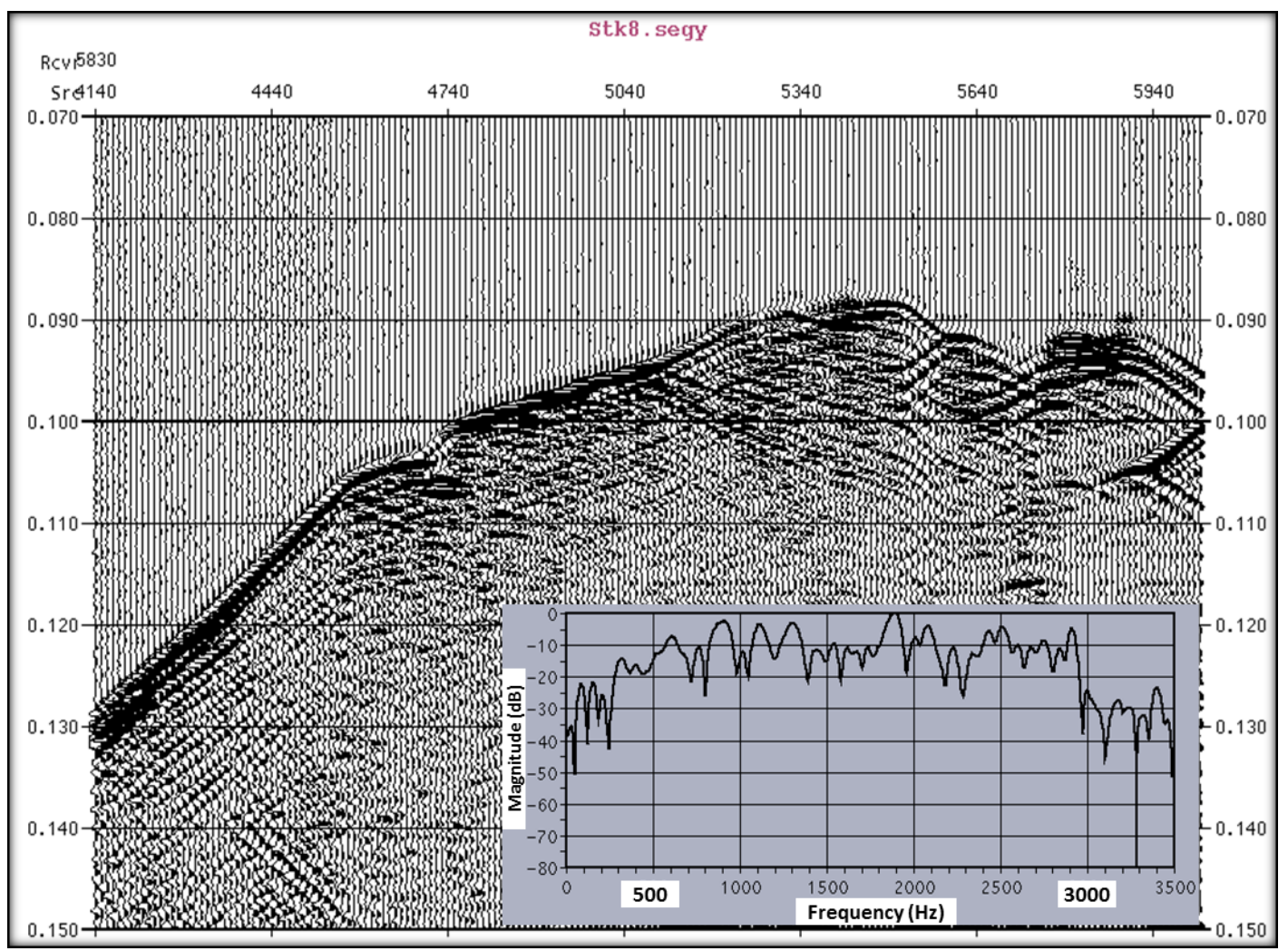

Figure 2.3: Common-receiver gather and spectrum for a receiver at $5830 \mathrm{ft}$ depth, and sources ranging from $4140 \mathrm{ft}$ (at left edge) to $6000 \mathrm{ft}$ (at right edge). The arrivals are clear and distinct even in this gather, prior to stacking.

For Coldspring field, the survey was designed similar to Springdale data. The differences; both wells (Coldspring 4-29 and Coldspring 1-29) were within the reef itself, although they did not penetrate deep beneath the reef as for the first data set. Also, this reef is at higher pressure, but still below bubble point. It also contains a significant amount of carbon dioxide that had been injected, along with flue gases, for EOR purposes. A summary figure of data acquisition is shown in Figure 2.4, providing additional details.

The presence of tube-wave noise generated by very low flow rates through the perforations after the wells had been "killed" caused the data to be of somewhat lower quality than the first data set (Springdale), but it is still excellent. Also, the data in Coldspring was acquired with a different sweep of 100 to $2000 \mathrm{~Hz}$. One example of a common-receiver gather is shown in Figure 2.5. 


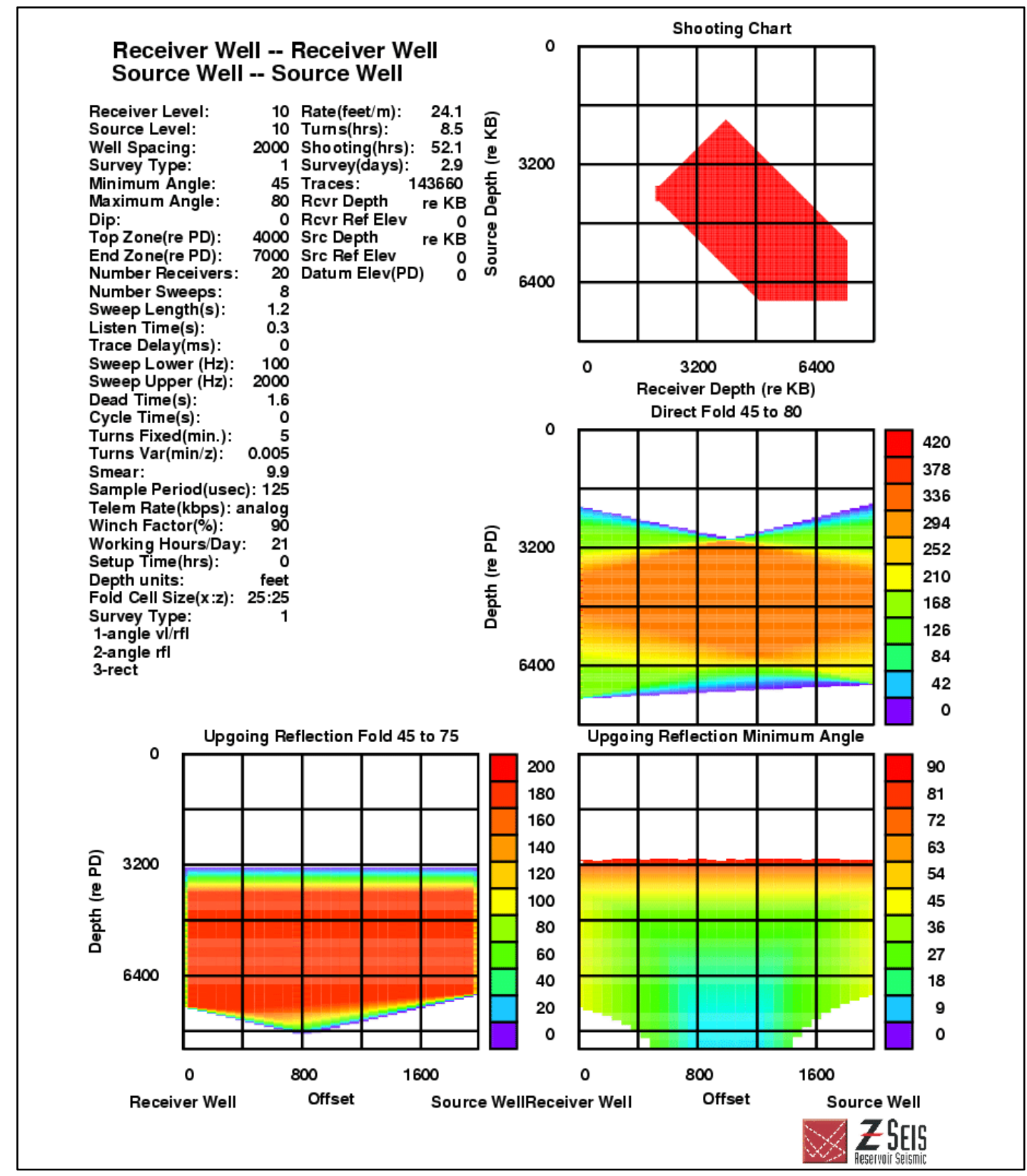

Figure 2.4: Summary of data acquisition in Coldspring field (courtesy Deep Look CS previously, Z-Seis). 


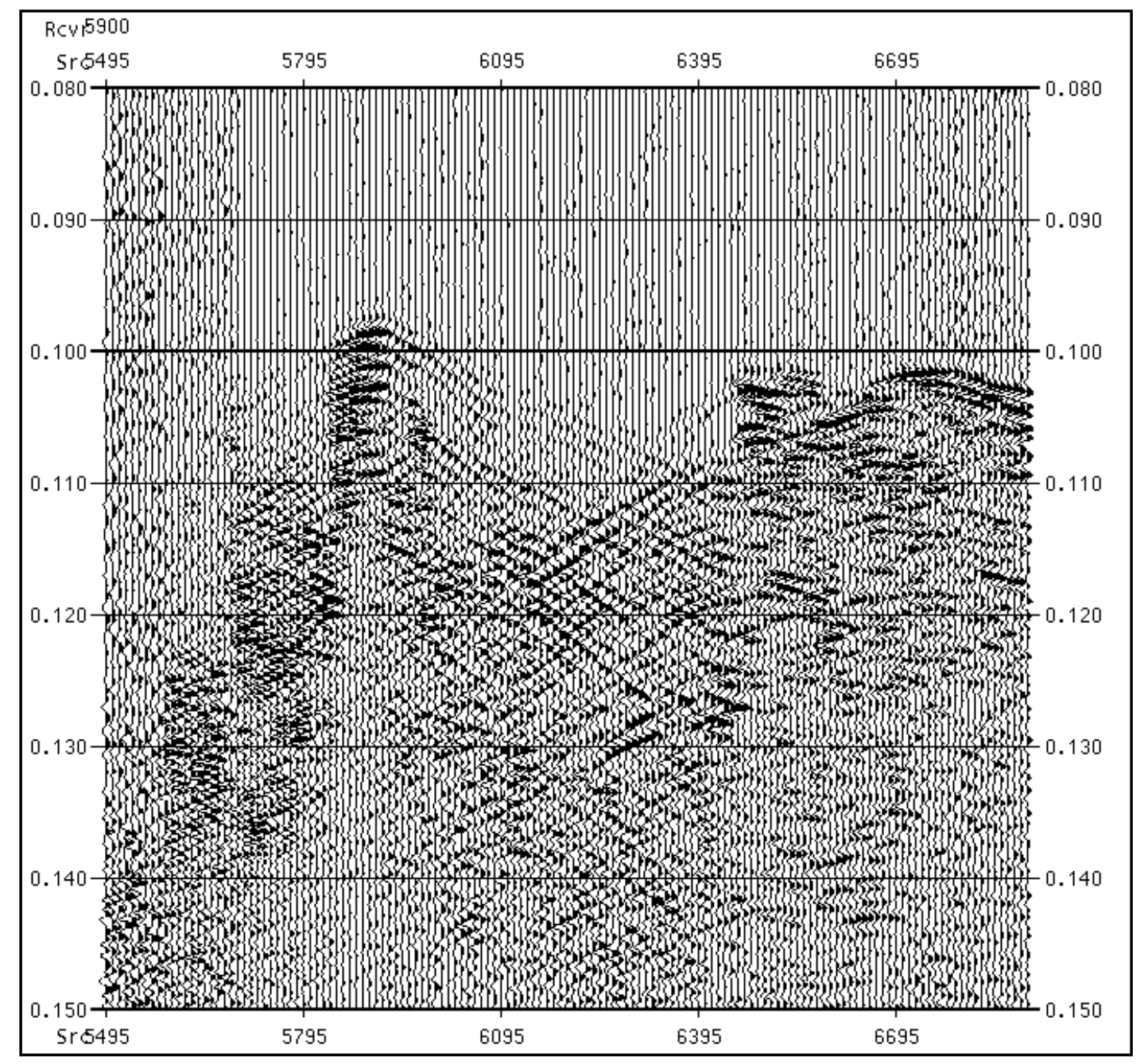

Figure 2.5: Common-receiver gather in Coldspring field for a receiver at $5900 \mathrm{ft}$ depth, and sources ranging from $5495 \mathrm{ft}$ (at left edge) to $7000 \mathrm{ft}$ (at right edge). The arrivals are clear and distinct even in this gather, prior to stacking.

For both fields, Springdale and Coldspring, the stacked images (figures 2.6 and 2.7) obtained are extremely high quality, and the prestack data can be used to examine and demonstrate the usefulness of such data for determination of lithology, fluid content, and pressure for compartments within the producing field.

Although crosswell seismic data can be used significantly to fill the gap in resolution over the conventional seismic methods to improve the vertical and lateral heterogeneity of the reservoirs but number of practical issues associated with crosswell reflection imaging can be observed such as; small angles of incidence are not possible, mapping issues, vertical and lateral stretch and velocity sensitivity. 


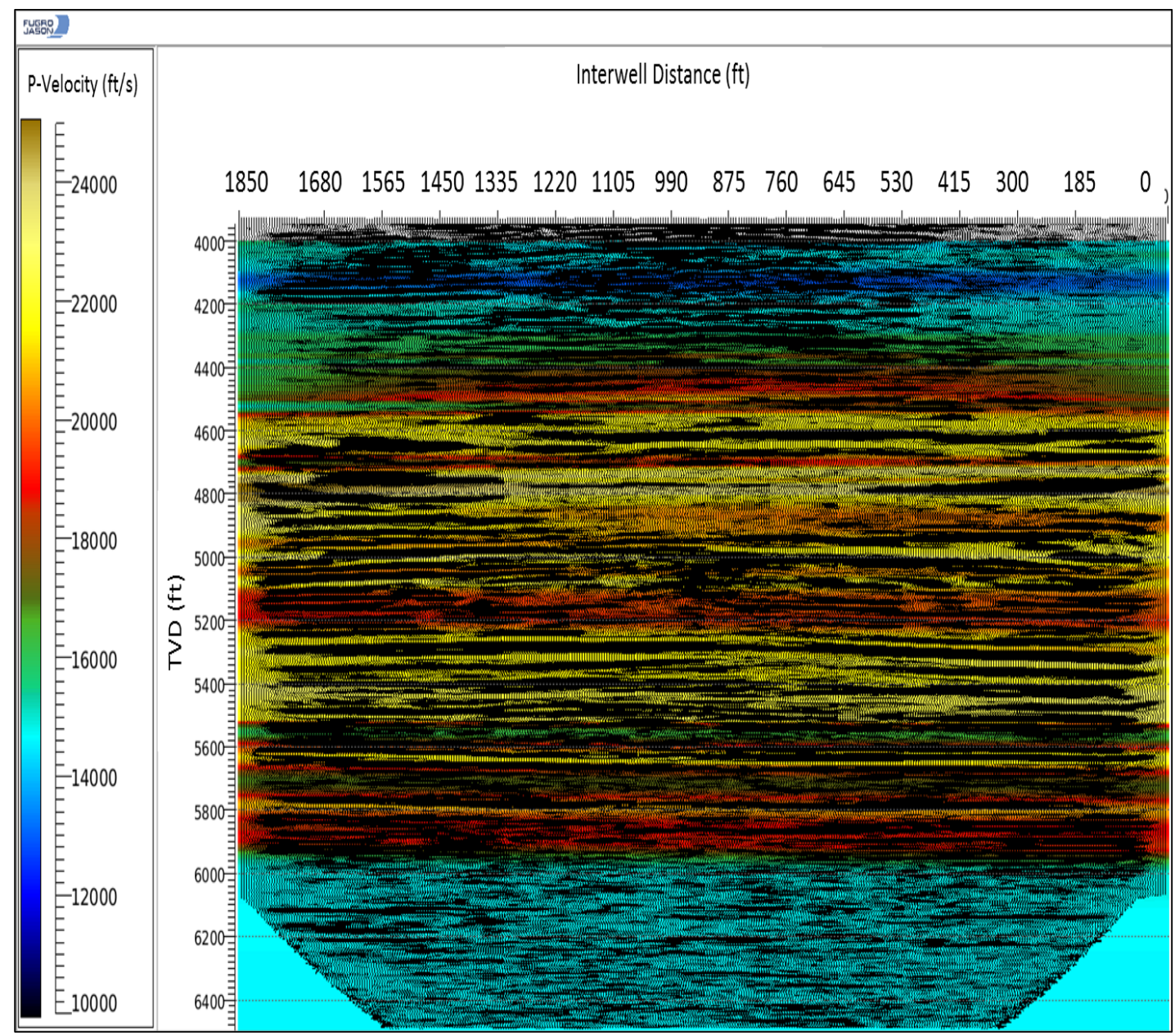

Figure 2.6: Migrated stacked image of Springdale crosswell data, with amplitudes held at constant gain across the entire image. Colors indicate the tomographically derived seismic velocities. The reef is readily visible as the low amplitude region at depths of about 4700 to $5000 \mathrm{ft}$ and extends from surface point 425 to $1335 \mathrm{ft}$. 


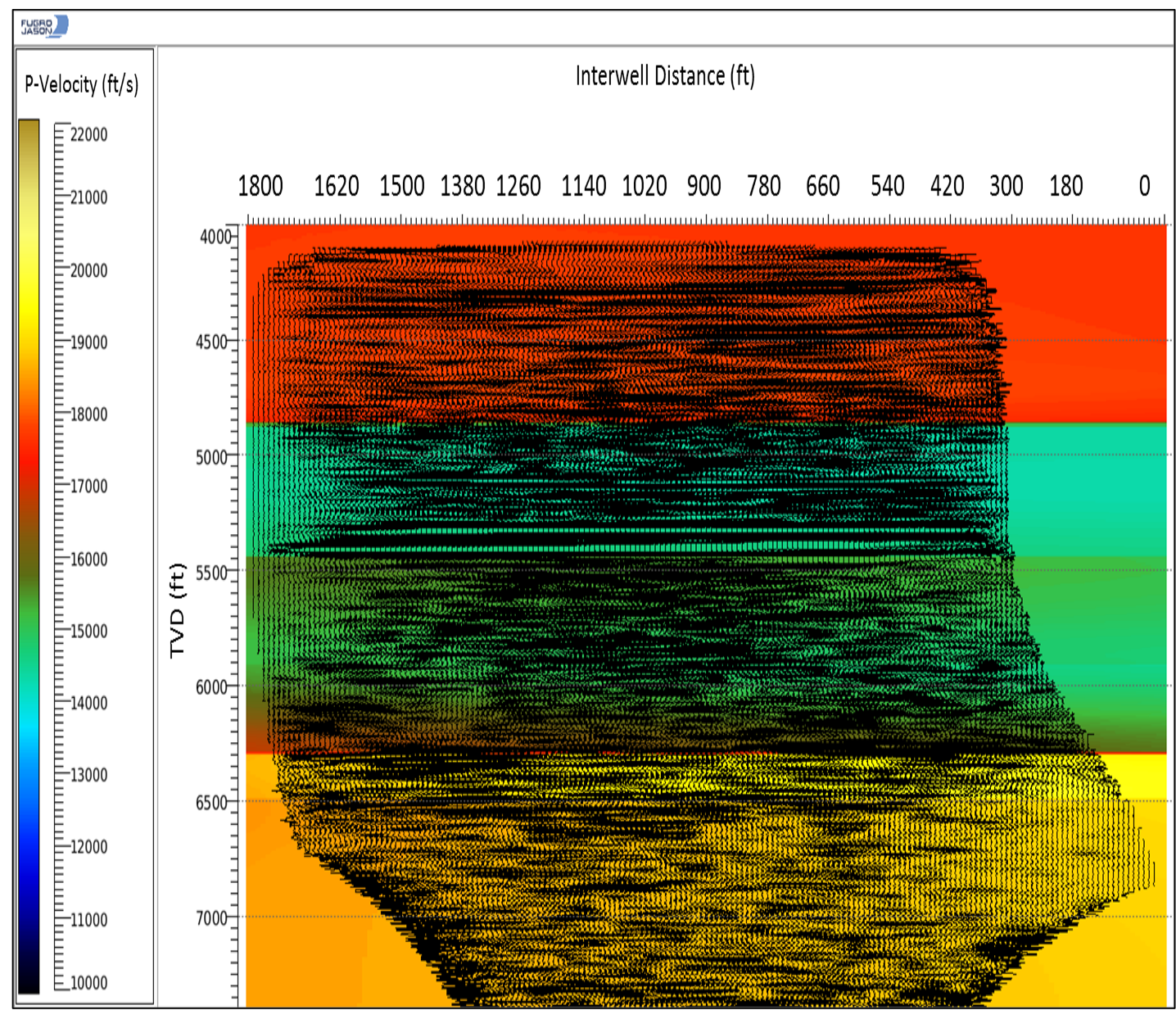

Figure 2.7: Migrated stacked image of Coldspring crosswell data (black wiggle traces), with amplitudes held at constant gain across the entire image. Colors indicate the tomographically derived seismic velocities. The reef is at depths of about 6700 to $7000 \mathrm{ft}$ and extends across the entire image.

In crosswell data, the stack image over the entire offset/angle range cannot be considered "zero-offset" or "full-stack" image as it's usually called in conventional seismic processing workflow. The fixed offset or separation of the source and receiver make small angles of incidence, in crosswell seismic, something are not possible. Also, the minimum observed angles in the data is always limited by the depth of the borehole as well.

The ray-theoretic imaging algorithm, the VSP-CDP (Vertical Seismic Profile to Common Depth Point) was first introduced by Wyatt and Wyatt (1981) and it has since been a very popular method of imaging in both VSP and crosswell data. The VSP-CDP mapping algorithm maps traces to curvilinear trajectories. Since the trajectories are not vertical, it introduces distortions to the signal's waveform similar 
to the stretch associated with the normal moveouts (NMO) correction. This distortion (stretch) is in both direction; vertical and horizontal stretch. Both types of stretches increase with angle of incidence. Meanwhile, the vertical stretch is independent of the location of the reflection point but the lateral stretch is largest close to the wells and decreases toward the middle (Lazaratos, 1993).

Although the mapping algorithm is accurate only for horizontal reflectors, it remains approximately valid for reflectors with small dip as shown by Lazaratos (1993). The mispositioning of reflection point due to dip scales with the interwell distance and does not depend on velocity. The lateral mispositioning is larger than the vertical mispositioning.

The mispositoning of reflection points due to velocity inaccuracies depends on the percentage of velocity error, not on its absolute value. The vertical mispositioning is smallest for intermediate angles and increases for small or large angles however the lateral mispositioning is largest for reflection points close to the wells and decreases to zero for reflection points located halfway between wells (Lazaratos, 1993).

The crosswell seismic data collected over carbonate reefs in Michigan to study the internal lithologic framework and fluid-phase distribution from the variation of seismic amplitude with angle of incidence. The challenge this approach faces is the strong dependence that both amplitude and phase have on the wide angles used in the crosswell environment. The benefits are derived from the extremely high resolution possible and the potential to image subtle variations using those very wide angles. 


\subsection{Carbonate Heterogeneity}

The target reef is belonging Silurian-Niagaran reefs located in the Northern Michigan reef and has a general trend of southwest to northeast line for approximately 170 miles and is 10-27 miles wide. These reefs have been important hydrocarbon producers in the State with a cumulative oil production of $475 \mathrm{MMBO}$ and $2.8 \mathrm{TCF}$ of gas (Wylie and Wood, 2005).

Reefs as oil and/or gas reservoirs can be extremely heterogeneous. Geologists model reefs with many internal layers and zones, and these can provide internal barriers to flow and isolate compartments from each other. Figure 2.8 shows one geological interpretation of internal reef structure while figure 2.9 shows a typical stratigraphic column for Michigan reef.

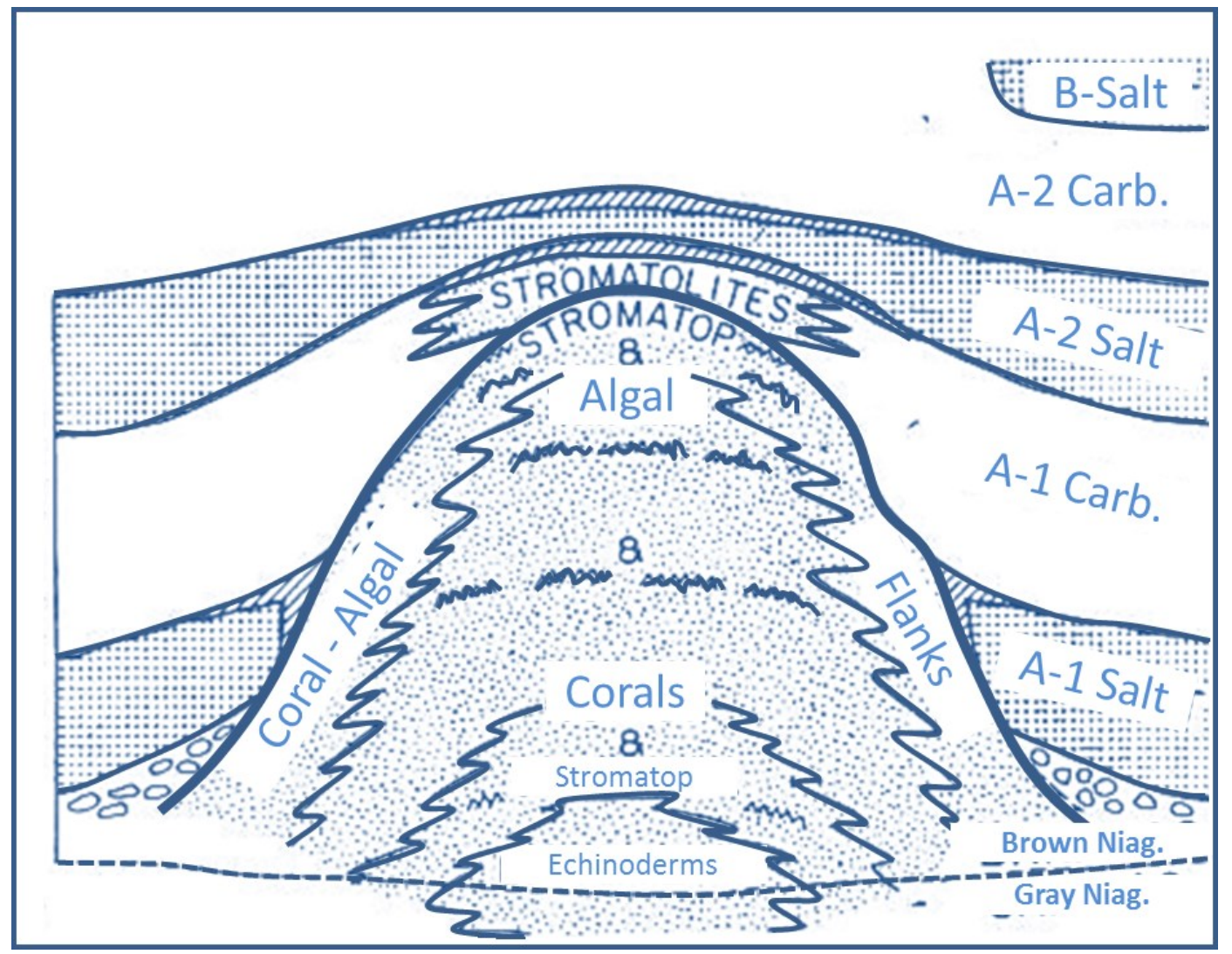

Figure 2.8: A typical geologic representation of a reef and its internal characteristics. Many of the facies identified are not likely to be productive in a hydrocarbon reservoir, and identification of theses facies will improve the recovery of oil significantly (modified from Droste and Shaver, 1985). 


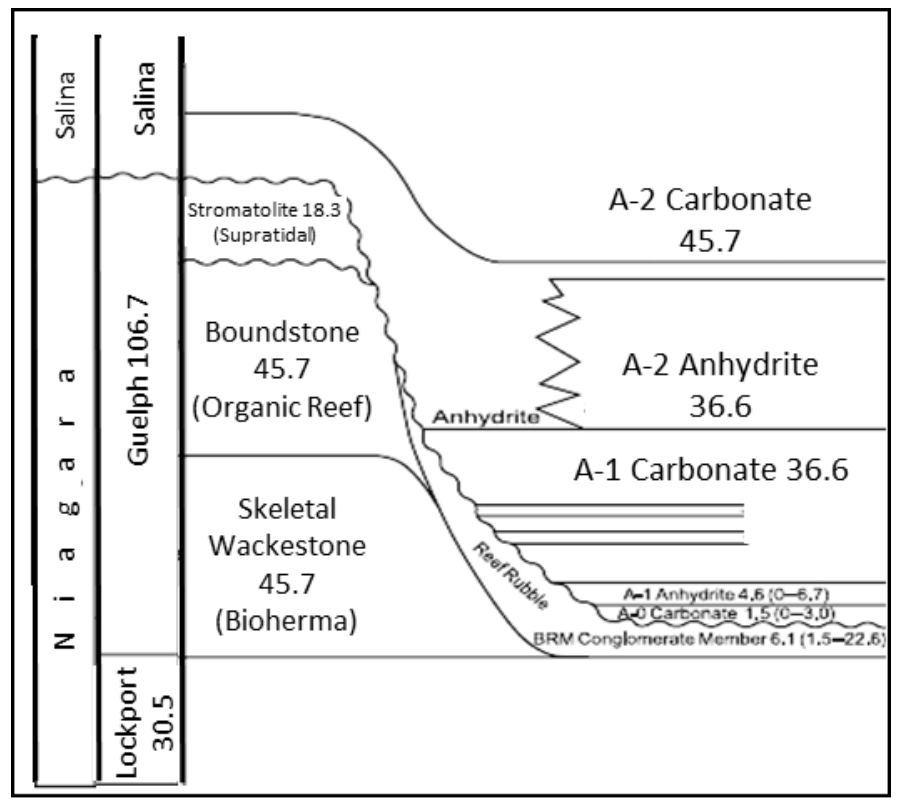

Figure 2.9: A typical Stratigraphic column showing subsurface nomenclature and correlations in the vicinity of the Belle River Mills, Michigan (modified from Wylie and Wood, 2005), with modifications. Average rock unit thicknesses and thickness ranges are shown in meters.

The literature is rich in geological studies of Michigan Basin reefs. The search for hydrocarbons in Michigan's southern and northern reef trends has produced an extensive library of literature (e.g. Sharma, 1966; Jodry, 1969; Huh, 1973; Mesolella, et. al. 1974, Gill, 1977; Shafer, 1974, Caughlin, et. al., 1976). Even the literature on reef terminology itself is voluminous (e.g. Cumings, 1932; Lowenstam, 1950; Pray, 1958; Nelson et. al. 1962).

Fortunately, nearly every author divides a pinnacle reef into a few vertically organized zones denoted by fauna. They all start with a carbonate mound or bioherm at the base upon which the reef grew. Mesolella uses "crinoidal" to describe that basal mound, then stepping up vertically he uses, "coral-algal", "algal", and "stromatoporoidal" to describe the remaining zones in a pinnacle reef.

Gill 1977, introduced a reef model that is divided into three stages or rock types: the top is supratidal (stromatolite), the middle is organic reef (boundstone), and the bottom is biohermal stage (wackestone). The organic reef stage as it divides into three zones: reef core, reef detritus, and vadose zone. The biohermal stage is divides into three zones: bioherm core, crinoid-bryozoa bioherm, and bioclastic detritus zone (figure 2.10).

Seismic imaging typically provides indications of contrasts in rock and fluid properties at a scale, is depending on the wavelength of the seismic signal. For typical 
surface seismic data in Michigan, the highest frequencies obtainable are generally less than $50 \mathrm{~Hz}$, and the seismic compressional-wave velocity for many of the carbonate layers is greater than $20,000 \mathrm{ft} / \mathrm{s}(6 \mathrm{~km} / \mathrm{s})$, resulting in wavelengths of $400 \mathrm{ft}(120 \mathrm{~m})$.

On the other hand, seismic frequencies from piezoelectric sources located within the earth, beneath the highly attenuating surface layer (glacial till in Michigan), as the case in crosswell surveys can result in the propagation of much higher frequencies, and therefore much smaller wavelengths. The resulting images from crosswell seismic should provide a better view of the reefs compared to surface seismic acquired any time. Figures 2.6 and 2.7 showed that the tops of the reservoirs can be clearly distinguished, and their lateral extent can be observed along the profile.

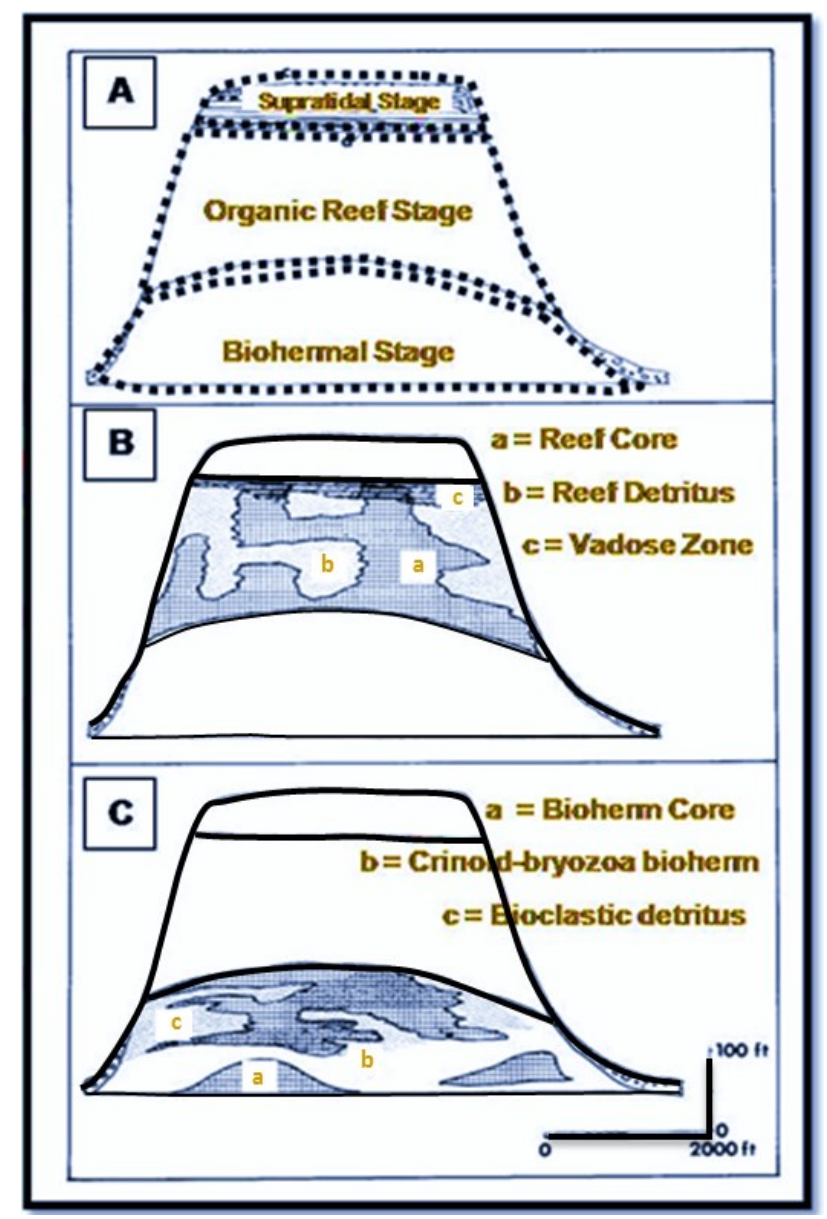

Figure 2.10: Lithofacies distribution pattern of Northern Michigan Silurian reef (modified from Gill, 1977). The reef as shown in " $A$ " is divided into three stages; Supratidal (top), Organic Reef (middle) and the Biohermal stage at the base. " $B$ " shows the Organic Reef and " $C$ " shows the Biohermal Stage. 


\subsection{Reflections from above versus from beneath}

In Springdale site two experiments were conducted at once: imaging "from above" in which the seismic source and receiver locations were above the reflectors, and imaging "from beneath" with tool locations beneath the reflectors.

It's worth mention that the reflections from beneath the interface does not contain angles as small as those from above the interface at the reef level, because the survey was not extend to much greater depths below the reef than necessary to image it (figure 2.11).

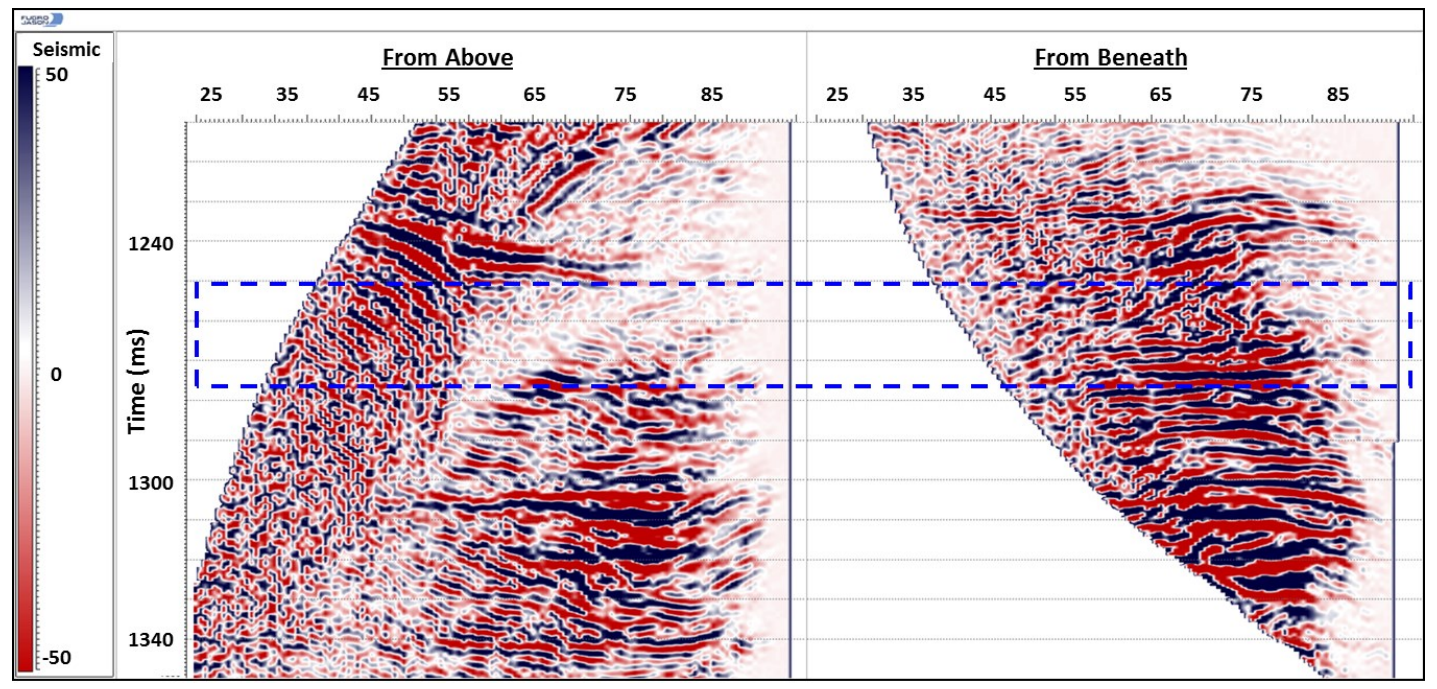

Figure 2.11: Angle gathers for data from above (left) and from beneath (right) in Springdale field. Notice the angle range for the data from beneath does not contain angles as small as those from above the interface at the reef level. The reef level is bounded by the blue dashed box.

Figures 2.12 and 2.13 are showing the stacked seismic and the amplitude spectra for the data sets from above and from beneath. It is very clear that the amplitude reflections from within the reef are low for both images. However, the one can notice the higher details within the reef in the stacked data from beneath compared to the one from above. Also, notice the attenuation zone that appeared below the reef depth and only recognized for the data from above (not recognized for the data from beneath). In addition, the amplitude spectra show that the image "from above" is not as high-resolution as the one "from beneath".

Generally, the high-frequency components of the seismic signal are attenuated more rapidly than the low-frequency components as waves propagate. Also, amplitudes are easily contaminated by many factors such as scattering, geometric spreading, source and receiver coupling, radiation patterns, and transmission/reflection effects. 


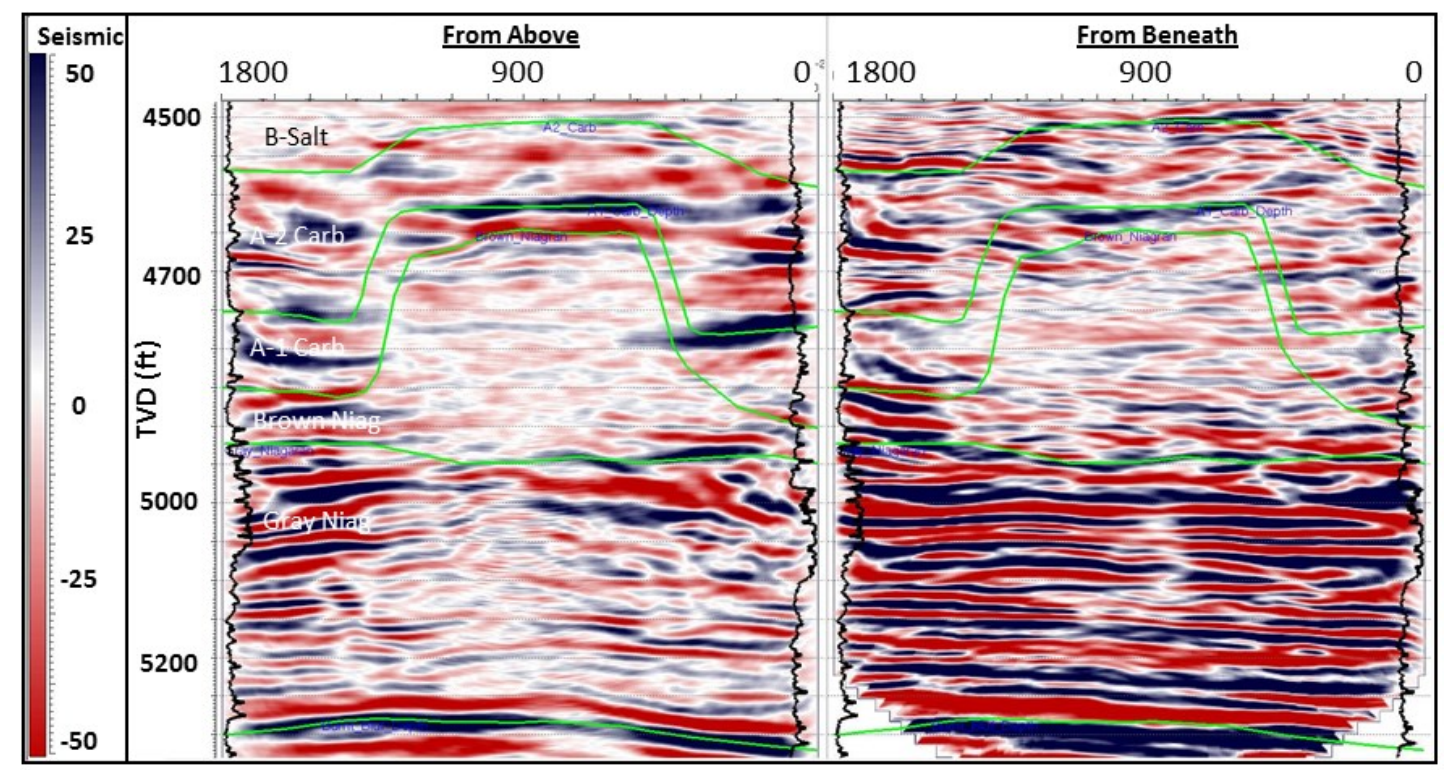

Figure 2.12: Seismic (stacked) section for Springdale from above (left) and from beneath (right). The image on the left shows the stack when sources and receivers are above the image, and the one on the right shows the stack when they are beneath the image. Notice the higher resolution within the reef in the stacked one from beneath (right). Seismic horizons are in green.

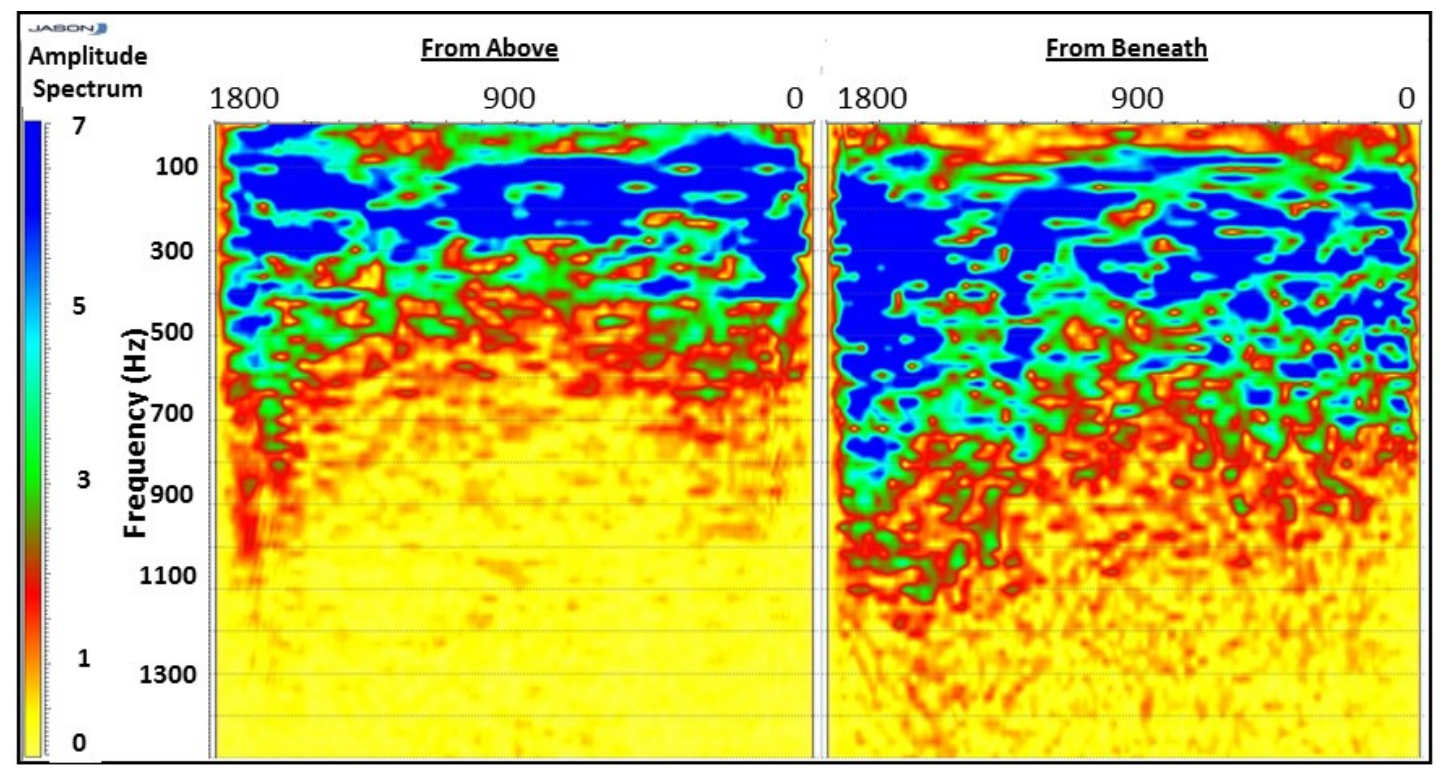

Figure 2.13: Spectra of seismic data from above (left) and from beneath (right). The time gate used to create this figure is within the reef only. This shows that the data recorded when sources and receivers are beneath the reef contains higher frequencies, allowing for improved imaging. 
However, since the reef appears to be an attenuating zone in both cases (from above and from beneath); this supports the notion that it is a property of the reef that causes the attenuation, whether it be fluid content/ low pressure causing intrinsic attenuation, or resulting high-density of scatterers.

The images created from source and receiver locations beneath the reef contain higher-frequency data within the reef, and appear to exhibit reflections that are more continuous than those imaged from above. We attribute this to attenuation and scattering of signal as it passes through the complex upper part of the reef, where gas saturation likely varies locally, reservoir pressure is extremely low, and structural complexity of the reef is evident. The image from beneath does not pass through this complexity and only experiences the gas saturation and low pressures at the upper edge of the image, allowing most of the reef image to be higher quality.

In addition, for most interfaces, the compressional-wave velocity increases with depth, and the data from above therefore encounter critical angles and associated post-critical phase rotations more often than the data from beneath (figure 2.14). Geometric constraints imposed by the borehole locations also result in angle-range differences in the images from above and from beneath.

The main exploration problem for carbonate reef reservoirs is determining the porosity distribution and evaluates the heterogeneity signature of the reservoir. Such an evaluation would improve the reservoir characterization and perform a number of reservoir development options.

This project is designed to provide the technique that makes the best-possible image of the interiors of two different Niagaran reef reservoir, using crosswell seismology, Amplitude-Versus-Angle (AVA) analysis and Inversion approaches. Since the AVA characters must result from identical contrasts in elastic properties in the two sets of images, albeit in reverse order, this project will provide an inversion approach to use the data from above and from below simultaneously at Springdale. 

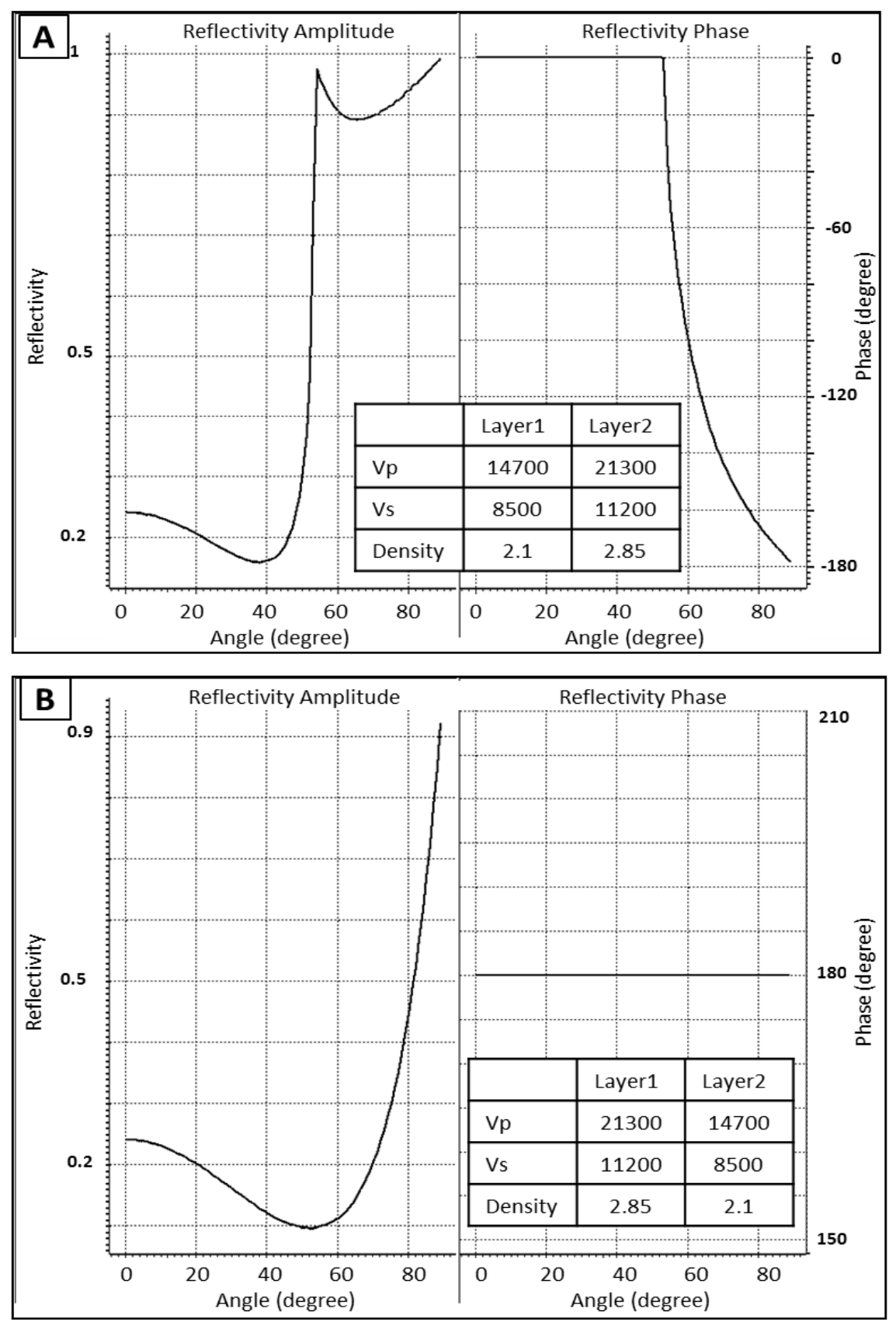

Figure 2.14: Zoeppritz solutions for a two layers model from above (a) and from beneath (b). P-and S-velocity values are in $\mathrm{ft} / \mathrm{s}$ while density is in $\mathrm{g} / \mathrm{cc}$. Notice the reflections observed from above encounter critical angles and associated post-critical phase rotations. For the reflectivity from beneath and since the second layer has lower impedance contrast, the reflections from beneath encountered no critical angles and most of the energy is transmitted into the second layer. This should be a quite often scenario for both data sets as the velocity tends to increase with depth. 


\section{Methods}

\subsection{Forward Modeling}

Forward modeling techniques in seismology begin with the numerical solution of the equation of motion for seismic waves. In other word, we solve for the numerical computation of theoretical or synthetic seismograms, for a given geological model of the subsurface. The objective is to compare the synthetic traces with the real seismic data acquired. If both agree to within acceptable level of accuracy, the initial geological model can be considered a reasonably accurate model of the subsurface. Usually, the term 'residuals' which is the difference between the synthetic and seismic traces are used to measure degree of the match between them. If the agreement is not good, the geological model is altered, and new synthetic traces are computed and compared with the data. This process continues iteratively until a satisfactory match is obtained between the synthetics and the real data.

There are numbers of computational methods that are commonly used to compute the synthetic seismic traces, such as ray theory, the finite difference, and finite element method. All the methods have their individual advantages and disadvantages, and an in-depth modeling effort for a given data set can involve the use of several methods.

Ray-tracing as a forward modeling technique is widely used in seismology for decades, for calculating seismic travel times, ray-paths, and synthetics. The raytracing algorithm is an iterative process that builds the ray path step by step using linear segment increments through a model where the velocity changes. Bending the rays at the layer boundaries is according to Snell's law, thus the ray path is a set of discretized points with appropriate information of incidence angles. For crosswell survey, the ray-tracing technique with a reasonable velocity model can calculate the angle coverage (min and max) of any reflectors between the source and reviver wells.

In figure 3.1 the ray paths over the velocity model at Springdale site are shown. The black ray path lines are reflected over a CDP point, a $900 \mathrm{ft}$ off the left well, over a reflector located at depth around $5300 \mathrm{ft}$. At any reflection point along the interwell space, the minimum and maximum angles of incidences are shown in figure 3.2.

Analyzing the nature of some AVA responses of crosswell seismic is difficult to do for couple of reasons; the non-uniqueness of AVA character itself and in part because of the crosswell data covers different, limited ranges of angles at different locations within the interwell space. Because different interwell locations contain different ranges of angles, ray tracing will be provide a good tool to calculate the angle ranges at each location. This would include the minimum and maximum angles expected at this particular location. Of course, the same idea can be extended to compare the 
angle ranges for the data from above versus the data from beneath at any location within the interwell space.

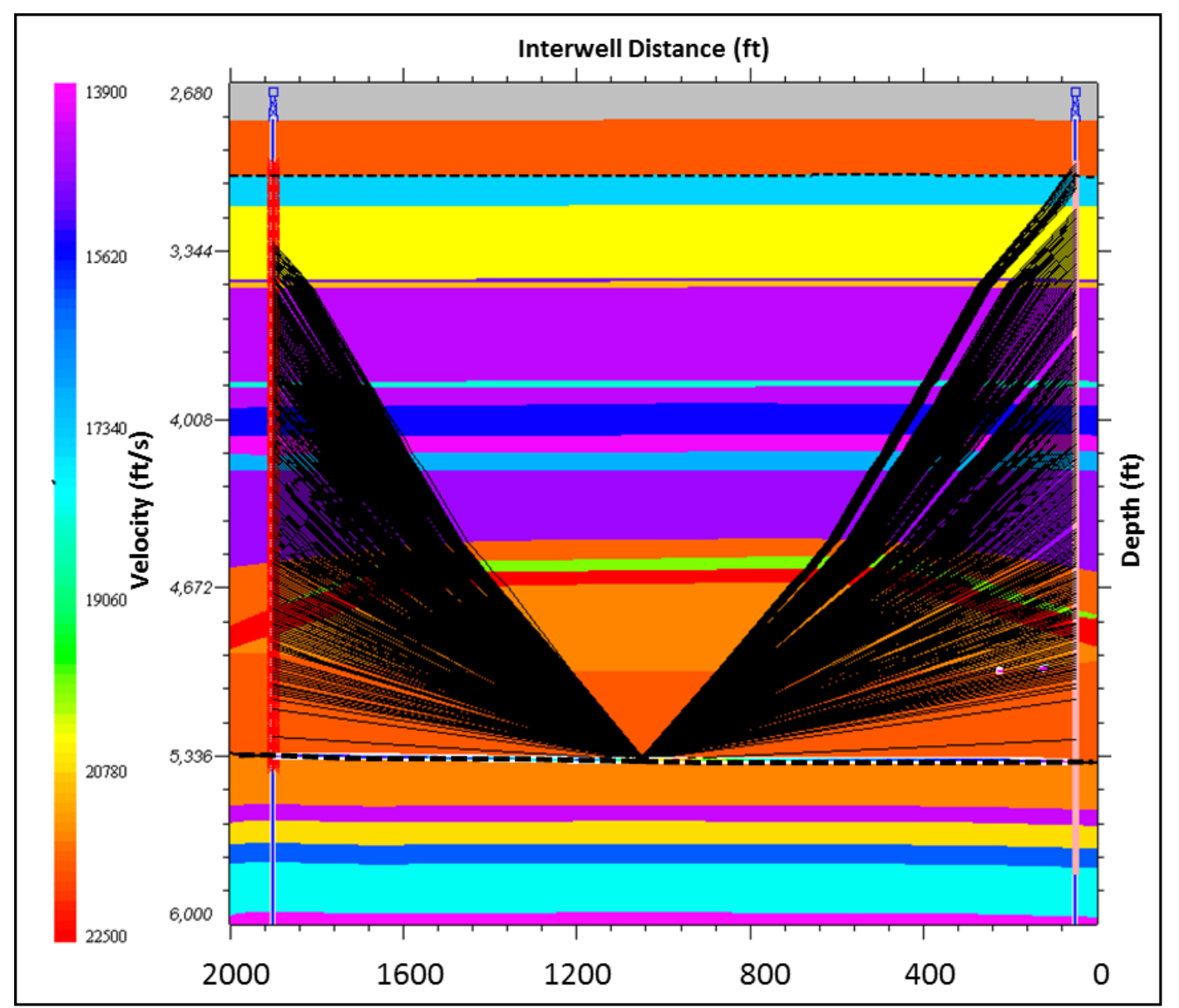

Figure 3.1: Velocity model (background colors) and ray paths (black lines) between two wells; one for sources (left) and one for receivers (right). The reflection point demonstrated here is $900 \mathrm{ft}$ away from source well. The $x$-axis is the interwell distance while the $y$-axis is depth in $f t$. 


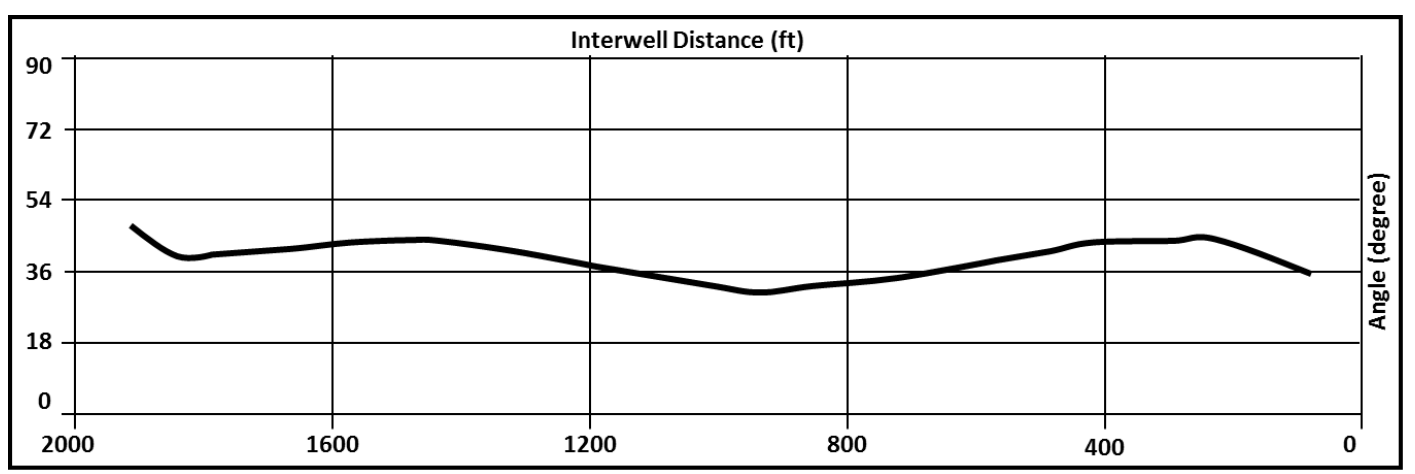

Figure 3.2: Minimum angle ranges as a function in the interwell distance. The figure is based on ray tracing for the reflector shown in figure 3.1 at depth $5300 \mathrm{ft}$.

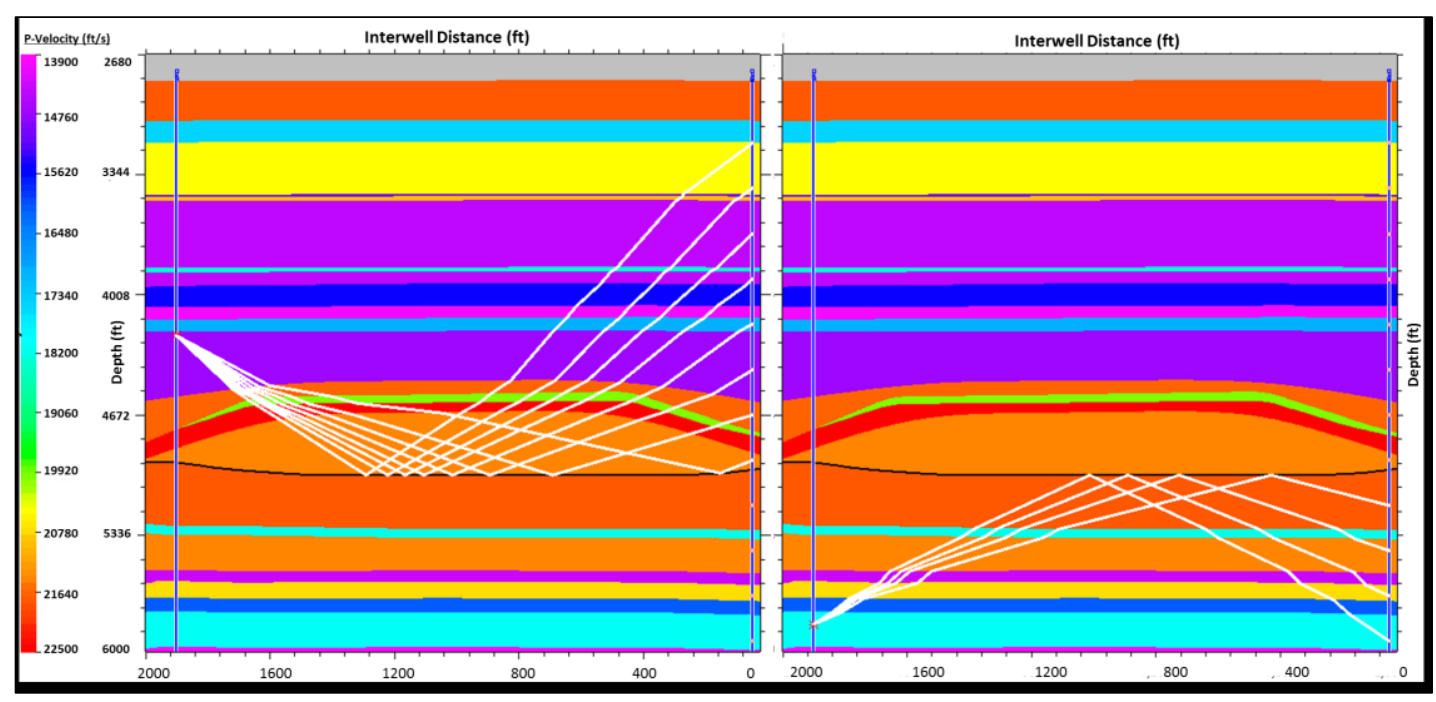

Figure 3.3: Ray tracing from source and receivers above (left) and beneath (right) the reflector. The reflector is at the base of the reef.

\subsection{Amplitude-Versus-Angle (AVA)}

Whereas most surface seismic data (and all standard analysis techniques) are used over reflection angles of $30^{\circ}$ or less (at most $45^{\circ}$, almost all of the data within a crosswell seismic set is at angles of $40^{\circ}$ or greater. It has been the experience that the processing that yields optimal images for crosswell data consists of narrow-angle gathers -as close to $40^{\circ}$ as practical; this is probably due to the wavelet distortion that occurs at wide angles, and selecting too broad an angle range will tend to stack wavelets of different phases as well as including multiple reflections and refractions, which are difficult to separate at these angles (e.g., Smith, 1993). 
Zoeppritz in 1919 derived a relationship that determines the amplitudes of the reflected and refracted waves at a plane interface for an incident P-wave (Sheriff and Geldart, 1995). This relationship describes the reflection and transmission coefficients as a function of angle of incidence and three parameters (Vp, Vs and Density) on each side of the reflecting interface Solving Zoeppritz equations involves solving a matrix of four equations.

Various linearized approximations to the Zoeppritz equations can be found in; Aki and Richards (1980), Shuey (1985), Smith and Gidlow (1987), Fatti et al. (1994) and others; these always assume small contrasts in elastic parameters and small angles of incidence (up to $30^{\circ}-35^{\circ}$ ), and they fail as the critical angle is approached. These limitations do not exist for the exact Zoeppritz solution although the exact solution itself is a plan wave approximation. While the critical angle is exclusively dependent on the P-wave velocity ratio across the interface, the post-critical reflections include reductions in P-wave reflection amplitude (due to shear-wave mode conversion), phase shifts, and appearance of critically refracted or head waves.

For crosswell seismic, using the approximations to Zoeppritz solution always is not sufficient because of the wide angles involved $\left(30^{\circ}\right.$ to near $\left.90^{\circ}\right)$. For a seismic wave impinging on an interface across which the P-velocity increases, a "critical angle" exists, beyond which P-wave reflection is nearly total (the reflection coefficient has an amplitude approaching 1.0) and no refracted (transmitted) $\mathrm{P}$ wave exists. However, the phase of the reflected seismic signal is distorted, in a way that can be predicted. Figure 3.4 shows the amplitude and phase of the reflected signal for such a seismic wave, from the complete (not approximated) solution to Zoepprittz equations. 


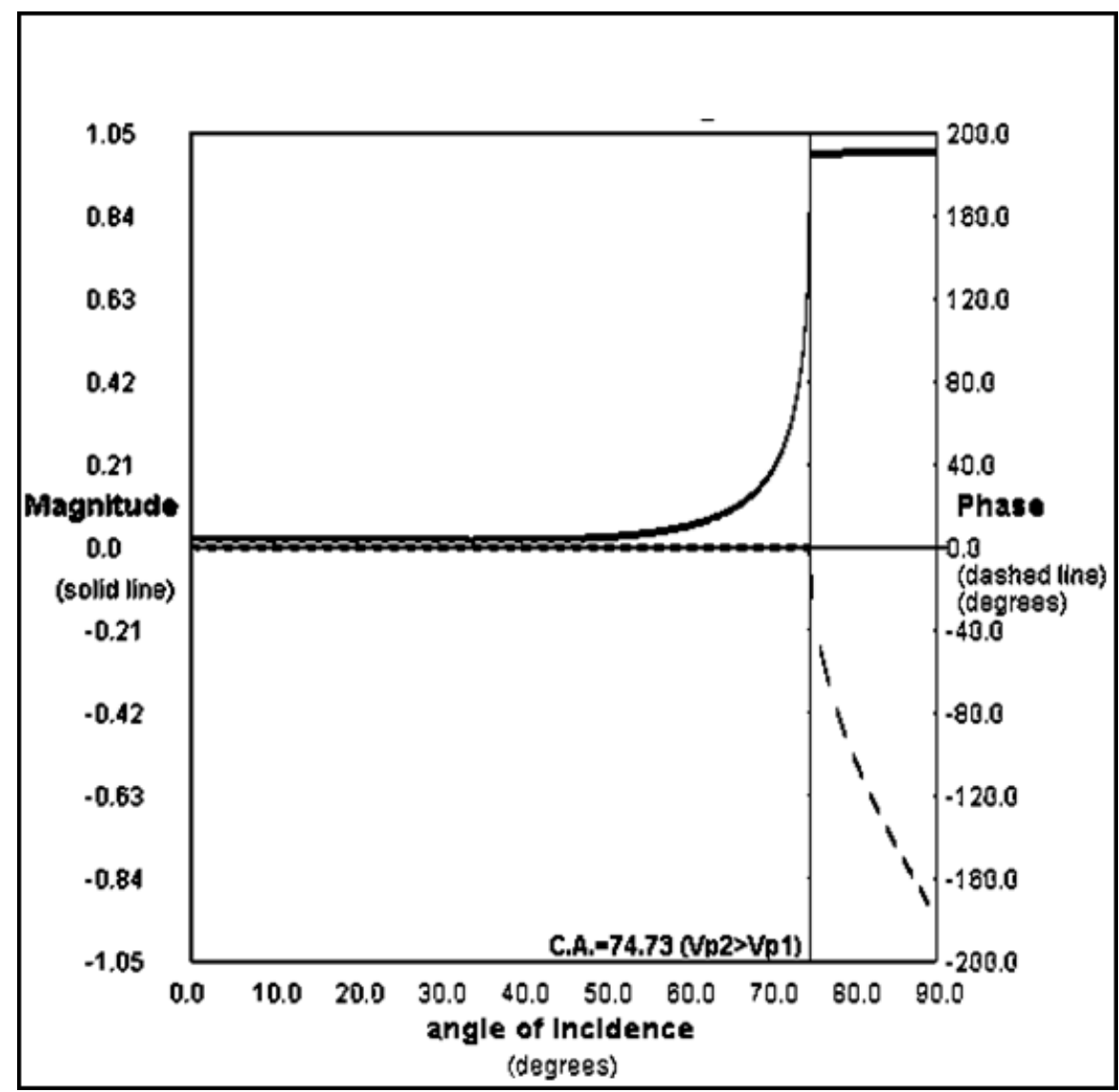

Figure 3.4: Amplitude (solid line) and phase (dashed line) responses of a typical full Zoepprittz solution (complete not approximation) for the reflector between the Brown and Gray Niagaran (This and other similar figures are adapted from the "Zoeppritz Explorer" applet available on www.crewes.org.). Notice the wide angle involved in crosswell data. In this example, the critical angle is seen at about $74^{\circ}$. Zoeppritz approximations that are limited to small angle of incidence will not be sufficient for crosswell data. Notice the amplitude and phase changing near and beyond the critical angle.

The amplitude of a reflection associated with the compressional $(\mathrm{P})$ wave incident at an interface varies as function of the angle with which it strikes that interface. The variation is determined by the compressional and shear wave velocities and the densities of the two formations creating the interface. Porosity, lithology, cementation, and saturation of the rock in turn affect these three properties. The variation in seismic energy with change in angles reflects differences in lithology and fluid content in rocks. AVA analysis (Amplitude-versus-Angle, also commonly called AVO or Amplitude-versus-Offset) is a technique by which geophysicists attempt to determine thickness, porosity, density, velocity, and fluid content of rocks. 
In some environments, it is possible to observe direct indications of hydrocarbon content from AVA analysis. Usually, this is accomplished by noticing changes in the AVA and/or zero-offset reflection character along a given horizon - it may be observed to exhibit certain characteristics when the interface is underlain by a watersaturated formation, and different characteristics when the underlying formation contains light hydrocarbons. In order for this technique to work, the interface must separate rock units which are themselves relatively homogeneous, or vary in a reasonably predictable manner. In addition, the contribution that the fluid content of the pores makes to the seismic velocity of the rock is important - in general, less compressible (faster) rocks, show less dependence of velocity on fluid content.

Most of AVA studies is to measure the change in the $\mathrm{Vp} / \mathrm{Vs}$ ratio, $\mathrm{P}$-wave velocity $(\mathrm{Vp})$ to S-wave velocity (Vs), and this is usually dominated by changes in the $\mathrm{Vp}$ term. $\mathrm{Vp}$ is only loosely dependent on fluid content in carbonates, where the rock frame comprises a larger proportion of the system's compressibility than in poorly consolidated clastic rocks. However, in the carbonate environments in which we conducted this study, the reefs are poorly suited for direct detection of hydrocarbons because the limestone and dolomite comprising the formation are highly incompressible and their velocities are very weakly dependent on fluid content. In addition, they are quite low porosity - a few percent at most - and the amount of hydrocarbon that may be present is therefore a tiny fraction of the total formation volume.

A complete analysis of AVA data often includes both detailed study of individual reflectors in pre-stack data and inversion of partial stacks, each of which cover a range of angles. This study is to be among the first to attempt to resolve lithologic and/or fluid information from such wide angles at such high resolution. Figures 3.4 and 3.4 show the AVA gathers at three different locations (CMP $=500,1000$ and $1500)$. Notice the angle ranges, of the data under investigation, from about $30^{\circ}$ to near $90^{\circ}$.

The reflections of beds within the reef between $1245 \mathrm{~ms}$ and $1272 \mathrm{~ms}$ (figures 3.5 and 3.6) are incoherent, especially for the images obtained from above. We attribute this to attenuation and scattering of signal as it passes through the complex upper part of the reef, where gas saturation likely varies locally, reservoir pressure is extremely low, and structural complexity of the reef is evident. The data from beneath does not pass through this complexity and only experiences the gas saturation and low pressures at the upper edge of the image, allowing the reflections to have higher degree of coherence. However, the reflections of beds below the reef (figures 3.5 and 3.6) are coherent. It appears that reflections whose ray paths pass through the reef itself are low-amplitude and somewhat incoherent, while those reflections whose ray paths pass outside the main body of the reef are more coherent. 


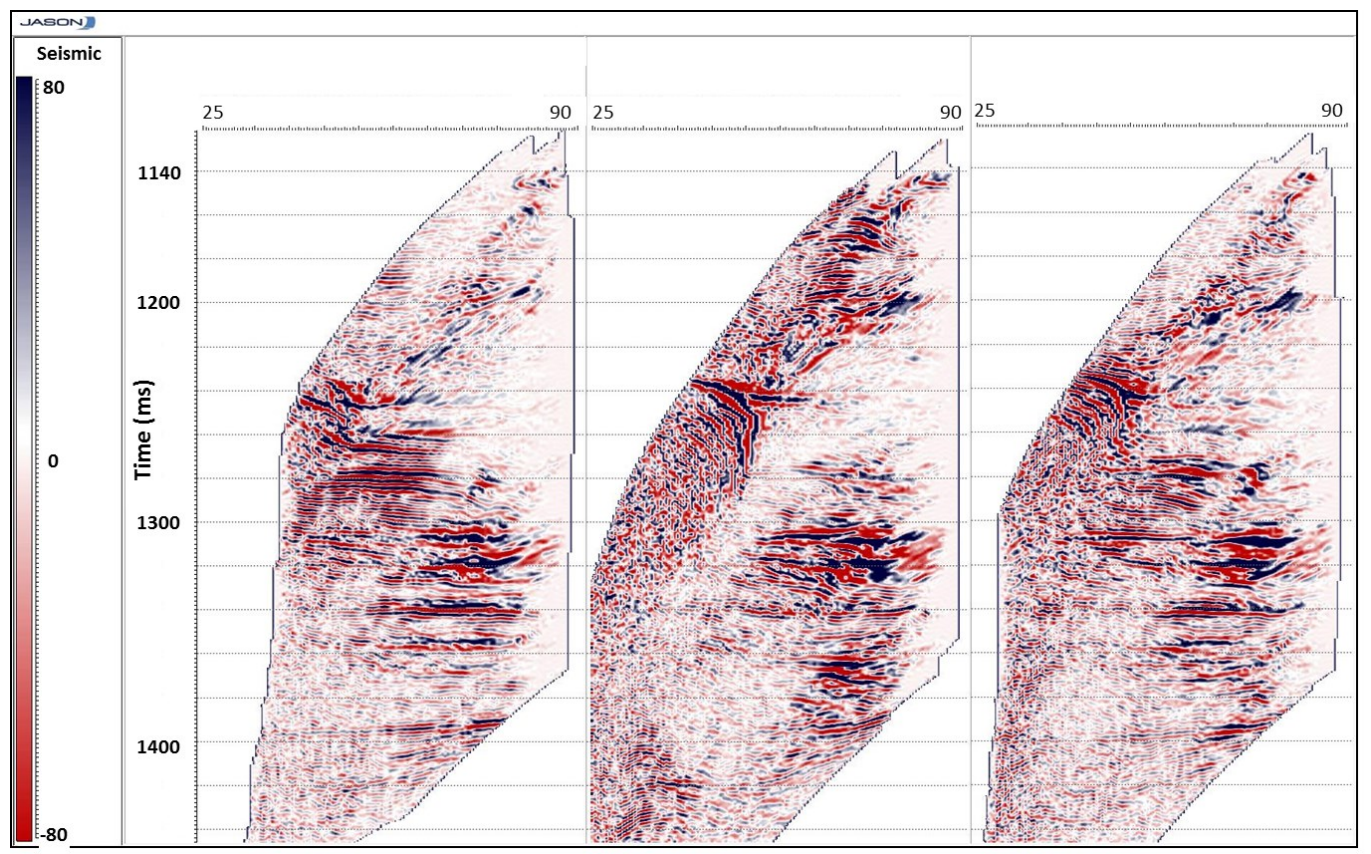

Figure 3.5: Angle gathers from above in Springdale data at three different CMPs; $C M P=500$ (right), 1000 (mid) and 1500 (left). The angles are $25^{\circ}-90^{\circ}$ from left to right on the horizontal axis. The reef area is from 1245 to $1272 \mathrm{~ms}$. Vertical axis is in time. 


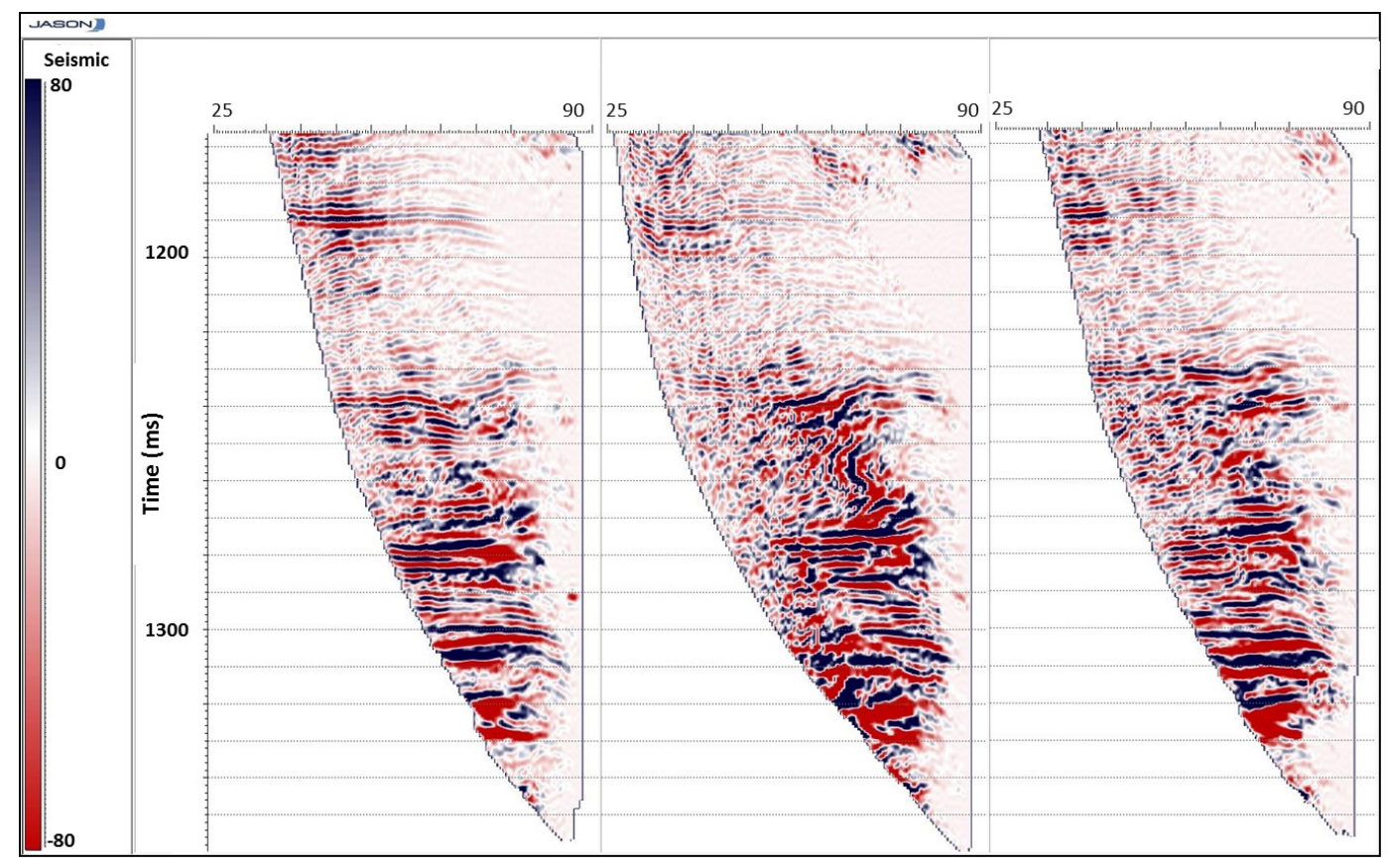

Figure 3.6: Angle gathers from beneath in Springdale at three different CMPs; CMP $=500$ (right), 1000 (mid) and 1500 (left). The angles are $25^{\circ}-90^{\circ}$ from left to right on the horizontal axis. The reef area is from 1245 to $1272 \mathrm{~ms}$. Vertical axis is in time.

We use the seismic gathers in detailed investigation of the amplitude variations with angle of incidence, identifying indicators of contrasts of physical properties at very wide angles. Examining the reflection character at different interfaces above and beneath the reef is one of the objectives. Then compare the AVA characters using the reflections obtained from sources and receivers above the reef with the same reflections obtained from sources and receivers beneath the reef. Also, studying the relation between the location of the reflectors whether beneath or above the reef and the direction of the ray path that go through the reef. Trisch (2006) studied the AVA character for three interwell distances beneath the reef for the same Springdale dataset and concluded that the angle ranges available at different locations varies greatly and limiting the range available for comparison at the different interwell locations.

For chosen reflectors, we wil compare the modeled solutions of full Zoeppritz equation with those from real data to examine the dependence of amplitude with angle of incidence and its character (figure 3.7). 

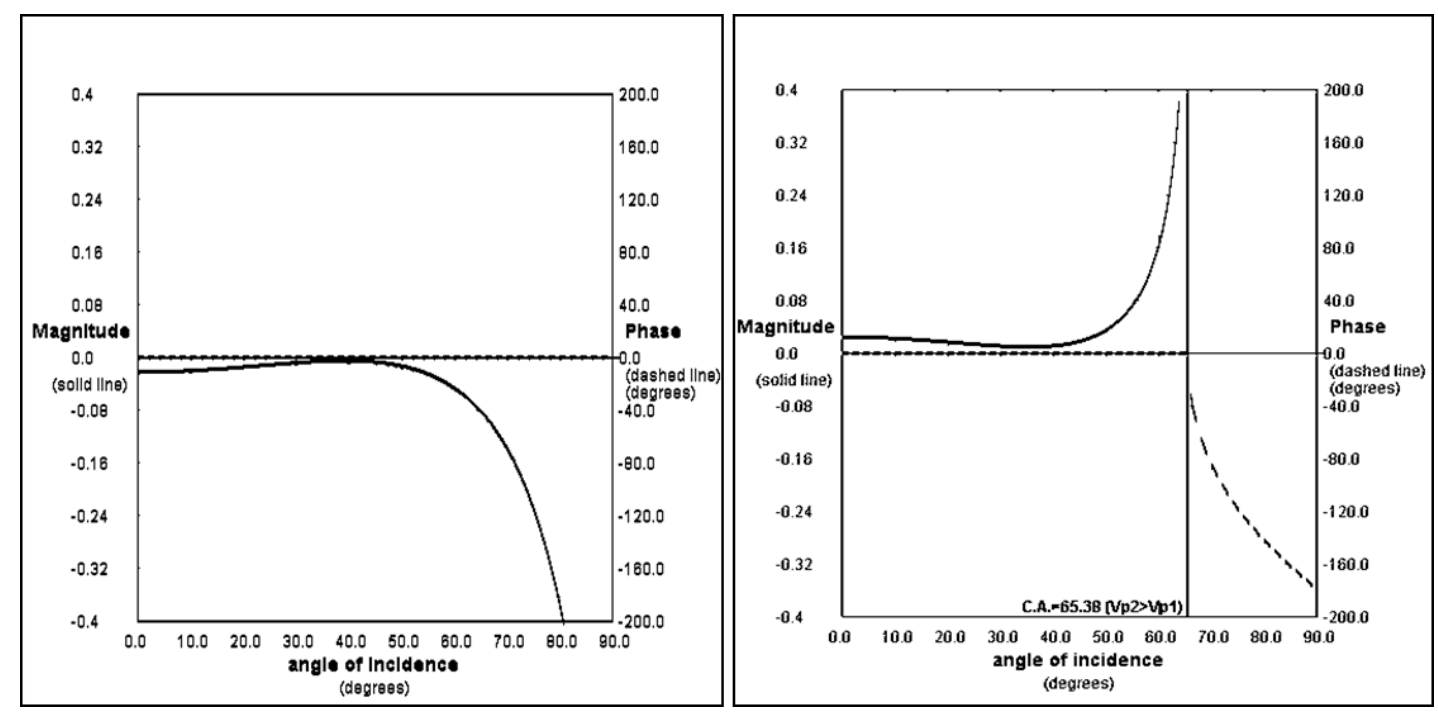

Figure 3.7: Two full Zoeppritz solutions (complete not approximation solution) for the same reflector (Brown Niagara plugged with Anhydrite, located inside the reef) from above (left) and from beneath (right) (modified from www.crewes.org.). The solid black lines are the amplitudes while the dashed line is the phase response. Notice the difference in AVA character, particularly beyond $40^{\circ}$.

Despite the fact that the crosswell seismic is high-frequency data that can provide extremely high-quality images of reservoirs, the interpretation of corsswell data itself sometime is difficult due to some issues introduced during the crosswell imaging steps. Among these issues are; the VSP-CDP (Vertical Seismic Profile to Common Depth Point) mapping that introduce stretching distortions to the data, the performing of mapping in the presence of dips, the migration artifacts introduced with the nonoptimum aperture as well as the sensitivity to inaccurate velocities. For these reasons, comparing the amplitude-versus-angle (AVA) gathers of actual crosswell data with exact solution of Zoeppritz equations will provide a better understanding for the crosswell data itself and will help in most detailed AVA studies required for reservoir characterization and reservoir management. In addition and since our study at Springdale includes the data "from Beneath" this will add another dimension of advantage to our AVA analysis and consequently to the interpretation of crosswell data. 


\subsection{Seismic Inversion}

Seismic samples contain two types of information - the time of each event and its amplitude. While the time of a given seismic event provides an estimate of the time or depth of a reflector, the changes in the amplitude provide an estimate of the lithologic variations in the sedimentary layers and (in certain situations) the presence of hydrocarbons. The changes in amplitude of reflections are caused by the impedance contrast that the seismic waves encounter across rock interfaces. Acoustic impedance is the product of density and P-wave velocity. The transformation of seismic data into impedance is commonly referred to as seismic inversion.

Inversion or "inverse modeling" is the opposite of forward modeling and it's the procedure of attempting to reconstruct subsurface model from a given set of geophysical measurements (figure 3.8). This involves removing the bandpass filter "wavelet" imposed by seismic acquisition and processing.

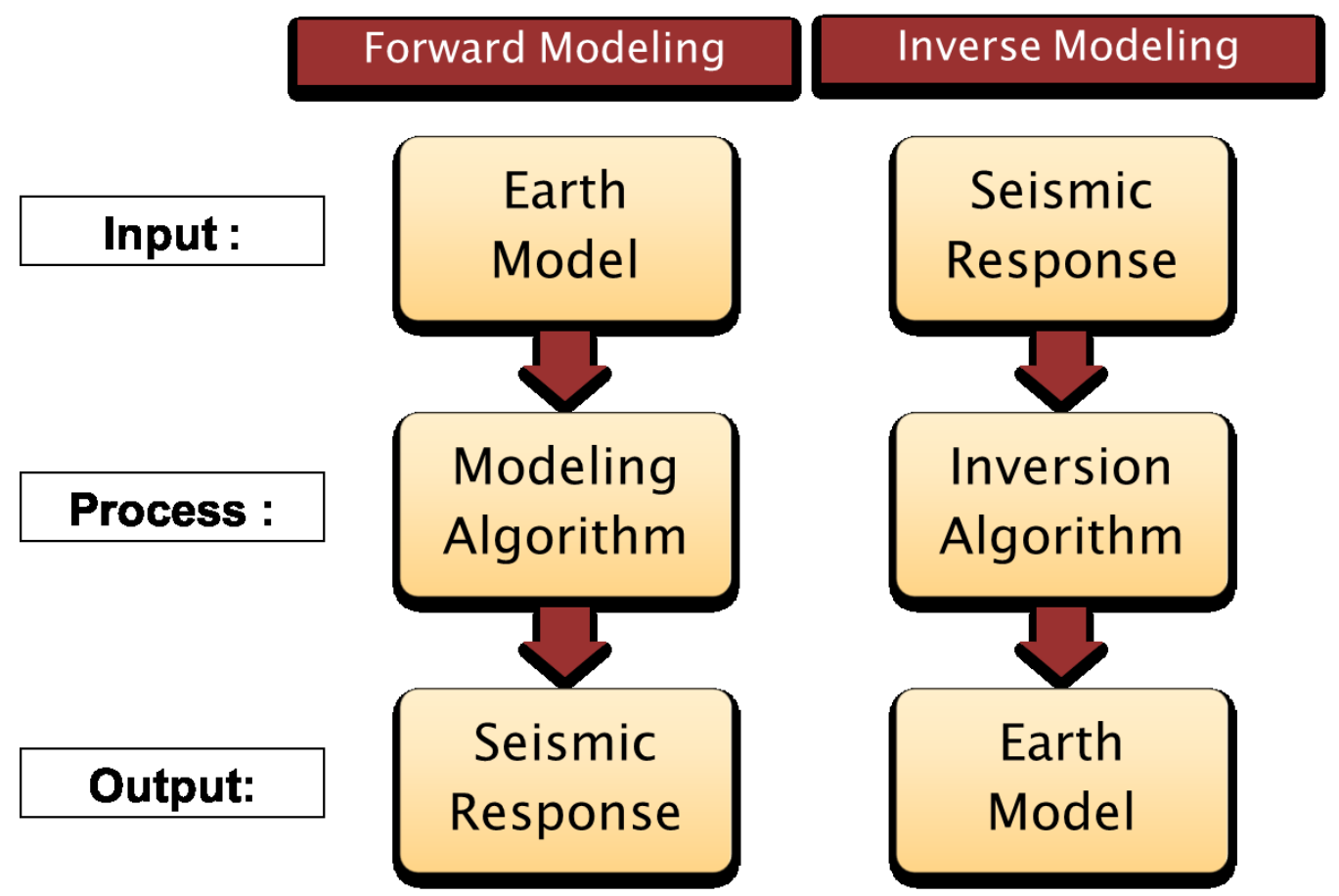

Figure 3.8: A schematic shows the forward and inverse modeling workflow. 
For forward modeling, we assume that we know the wavelet source and the reflectivity driven from the impedances of the vertical section and generate the synthetics seismic trace. We use a mathematical relationship such as the wave equation or asymptotic ray theory to synthesize the seismograms. Mathematically, the Green's function, maps changes in the impedance to the seismic trace through the use of the seismic source wavelet.

For inversion, we have the seismic trace as a measurement and we assume we know the wavelet that impinged into earth and then estimate the impedances (figure 3.9). In other word, the inversion always refers to the reverse process whereby we attempt to retrieve a geological model that adequately describes the seismic data.

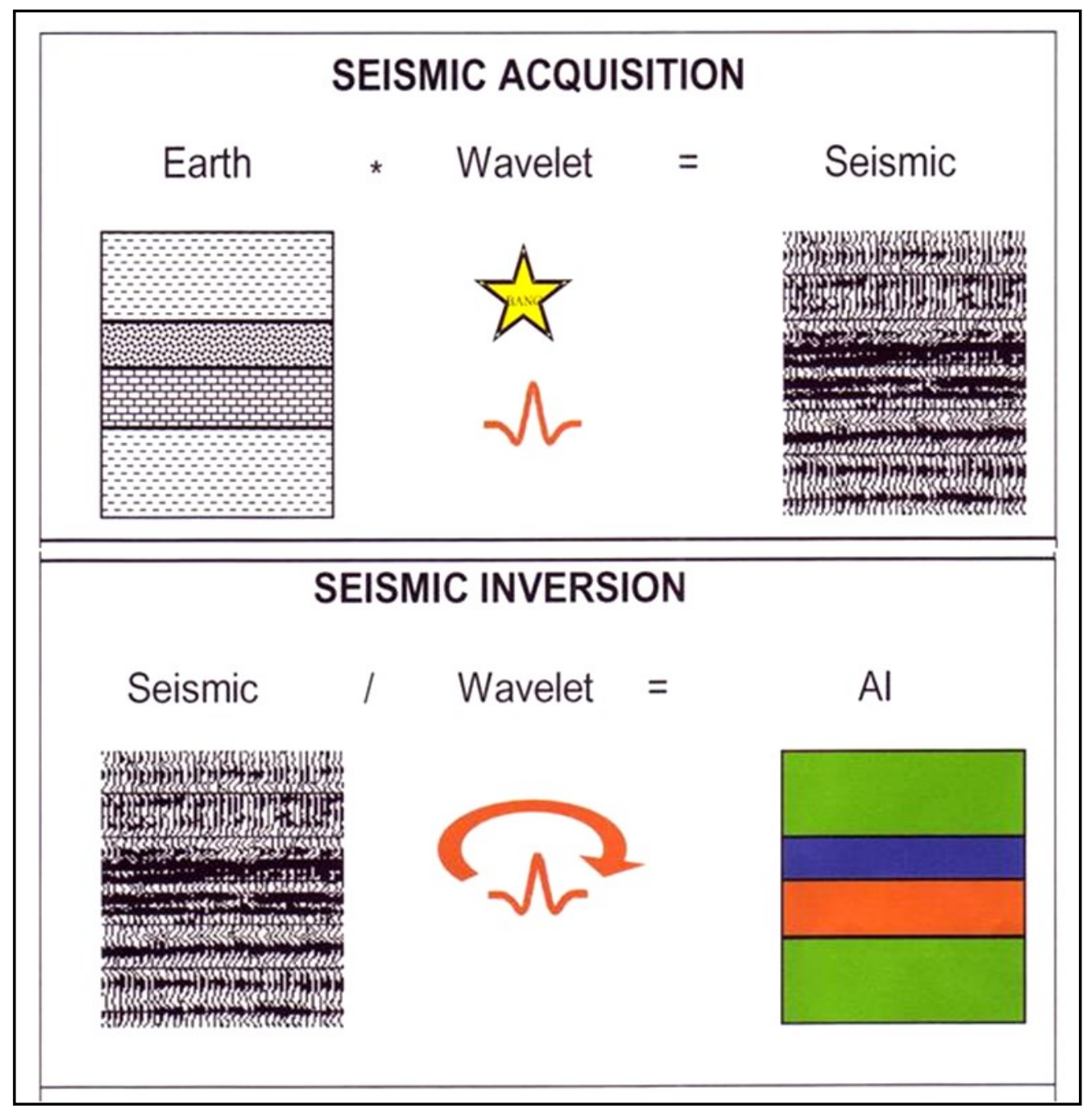

Figure 3.9: A schematic shows the difference between seismic acquisition and inversion. 
Seismic inversion for impedance is widely practiced in the industry (Latimer et al, 2000; Contreras et al, 2006; Pendrel, 2006; Pendrel et al. 2000). Normal incidence reflectivity $\left(\mathrm{R}_{0}\right)$ of compressional plane waves depends only on contrasts in acoustic impedance.

$$
\mathrm{R}_{0}=\left(\mathrm{Z}_{\mathrm{P} 2}-\mathrm{Z}_{\mathrm{P} 1}\right) /\left(\mathrm{Z}_{\mathrm{P} 2}+\mathrm{Z}_{\mathrm{P} 1}\right)
$$

Where acoustic impedance (Zp) is the product of density $(\rho)$ and seismic P-velocity $(\mathrm{Vp})$. The different rock layers are indicated by subscripts 1 and 2 across the interface.

$$
\begin{aligned}
& Z_{\mathrm{P} 1}=\rho_{1} V_{\mathrm{P} 1} \\
& Z_{\mathrm{P} 2}=\rho_{2} V_{\mathrm{P} 2}
\end{aligned}
$$

The compressional velocity (P-wave) is a function of three parameters: the bulk modulus $K$, the rigidity $\mu$ and the bulk density $\rho$. The shear velocity ( $\mathrm{S}$-wave) is a function only in the rigidity $\mu$ and the bulk density. We can write the following;

$$
\mathrm{V}_{\mathrm{P}}=((\mathrm{K}+4 / 3 \mu) / \rho)^{1 / 2}
$$

$$
\mathrm{V}_{\mathrm{S}}=(\mu / \rho)^{1 / 2}
$$

From equation 4 we see that the velocity of elastic waves represents a trade-off between the elastic firmness ( $\mathrm{K}$ or $\mu$ ) and density; typically, however, as a given rock type increases in $\mathrm{K}$ or $\mu$, density also increases.

In non-normal incidence where the data is affected by the concurrent mode of conversion from compressional to shear waves. The reflection coefficient will be defined as a ratio of amplitudes dependent on other parameters, such as the shear velocities and densities, and is described as a function of incident angle by the Zoeppritz equations. 
Elastic impedance (EI) is defined as the property of rock which produces reflections at non-zero incident angles, and it is an approximation derived from a linearization of the Zoeppritz equations. Connolly (1999) suggests an alternative expression for the reflection coefficients of $\mathrm{P}$ waves at non-normal incidence in the following form,

$$
\mathrm{R}_{\mathrm{pp}}(\theta)=\left(\mathrm{EI}_{2}-\mathrm{EI}_{1}\right) /\left(\mathrm{EI}_{2}+\mathrm{EI}_{1}\right)
$$

The subscripts 1 and 2 denote to the upper and lower layer. Connolly (1999) defined the EI is an angle-dependent weighted product of P-wave velocity, S-wave velocity, density:

$$
\mathrm{EI}(\theta)=\mathrm{V}_{\mathrm{P}}{ }^{(1+\tan 2 \theta)} \mathrm{V}_{\mathrm{S}}{ }^{(-8 \mathrm{~K} \sin 2 \theta)} \rho^{(1-4 \mathrm{~K} \sin 2 \theta)}
$$

Here $\mathrm{K}$ is used to represent the average $\mathrm{V}_{\mathrm{s}}{ }_{\mathrm{S}} / \mathrm{V}^{2}{ }_{\mathrm{p}}$ value over the two media above and below the reflector (and not the bulk modulus, as we used $\mathrm{K}$ in equation 3). Examination of equation 5 shows that the EI at zero degrees is the same as the Acoustic Impedance, as it should be: $\mathrm{AI}=\mathrm{EI}(0)$. Thus the Constrained Sparse Spike Inversion (CSSI) developed for AI inversion can be extended to be used also for EI inversion ( $\mathrm{Lu}$ and McMechan, 2004), using partial-stack volumes representing different angle ranges.

In the last two decades, inversion has proven extremely successful and became a standard part of the workflow for reservoir delineation and quantitative reservoir characterization. Many reasons for that; first, it reduces the tuning and interference effect of seismic wavelets and increases the bandwidth of the seismic data by adding the low frequency bands from well logs. Second, it transforms the seismic data from the amplitudes interface properties to layer properties that are absolute values for the rocks themselves. This transformation provides a quantitative measurement of the rock properties in the subsurface. Also, the constrained inversion suppresses noise. Hill (2005) compared thicknesses estimated from seismic amplitude and seismic impedance inversion and showed that impedance data extends thickness measurements below the classical tuning limits. Figure 3.10 shows the difference between the interpretation using the seismic amplitude section and the impedance layer property section. 


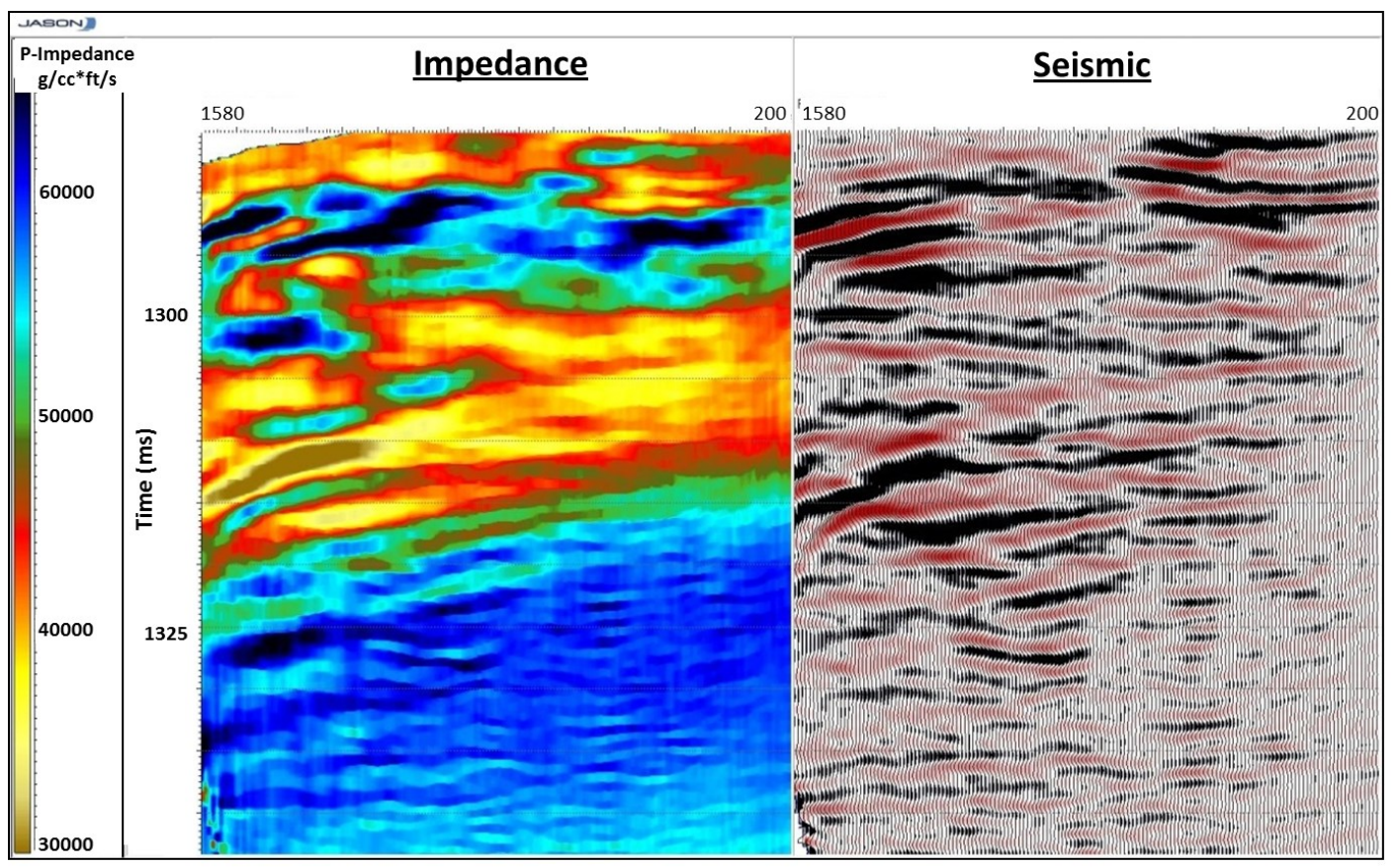

Figure 3.10: Acoustic impedance (left) and stacked seismic (right) for the same data set. Notice how nicely the layer boundaries are defined on the impedance section compared with the seismic section. This section is a close-up of a portion taken from Coldspring data.

Seismic reflection amplitudes are dominantly, but not exclusively, generated by contrasts in acoustic impedance. The standard practice of calibrating stacked seismic data to an acoustic impedance log ignores AVA effects by assuming zero-offset properties, thereby risking failure and missing an opportunity to better constrain the interpretation. Normal incidence reflection of compressional plane waves depends only on contrasts in acoustic impedance. In non-normal incidence the data is also affected by the conversion of the incident compressional wave to reflected and/or transmitted (refracted) shear waves. Thus the amplitude variation with angle (AVA) exhibited by seismic reflection events depends on the shear waves as well as the compressional waves and density. Consequently, AVA is related to variations in both p-impedance and s-impedance. S-impedance is not affected by pore fluids to the same degree as p-impedance, as the fluids do not contribute to rigidity. Pre-stack inversion makes use of this variation in reflected P-wave amplitude to extract additional information about the subsurface.

The input seismic data to inversion process can be of two types; full-stack (often assumed to be zero-offset, or "acoustic") or based on angle or offset stacks (non-zero offset, or "elastic"). The workflows to run the inversion are based on deterministic algorithms or geostatistical (probabilistic) algorithm; in this work we will use both types of inversion algorithm. 
The deterministic inversion of seismic amplitude data used in this work is based on constrained sparse spike inversion (CSSI) algorithm used in software provided by Fugro-Jason (Debeye and van Riel, 1990; Pendrel and van Riel, 1997). As mentioned earlier, this inversion can be of two types; acoustic (full-stack, considering this to be equivalent to zero-offset) or elastic (making use of non-zero offsets). Particularly in our crosswell data, the full-stack inversion is, of course, not acoustic because of the very-wide angles involved, so the resulting image (Figure 3.11) is referred to here as showing "apparent" impedance. As long as the angles included in the full stack do not include many critical-angle reflections, the resulting apparent impedance can be used as an indicator of porosity in these cases of carbonate reefs.

The deterministic simultaneous AVO inversion for partial stacks (non-zero offsets) used in this work is also based on the constrained sparse spike inversion (CSSI) algorithm but extended to AVO domain.

The CSSI inversion creates a set of elastic property volumes from multiple seismic partial (angle or offset) stacks. At each CMP the seismic is modeled as the convolution of a set of reflection coefficients with the wavelet. The reflection coefficients are derived from elastic parameters using a full solution for KnottZoeppritz equation. The inversion process is controlled by a set of constraints based on the prior information from the known geology and from well logs. These constraints effectively limit the range of potential solution to those that have geophysical and geological significance. The lateral variations in the low frequencies are incorporated through the use of elastic parameters volumes (from well logs and/ or from scaled seismic velocity) as the trend. The inversion process begins with estimating the elastic parameters contrast that create synthetic simultaneously honor all the input partial stacks. Then these elastic parameter contrasts are integrated to create elastic parameter volumes. The inversion process is accomplished when the elastic parameters are optimized by modifying the low frequency trends and enforcing compliance with any additional constraints. 


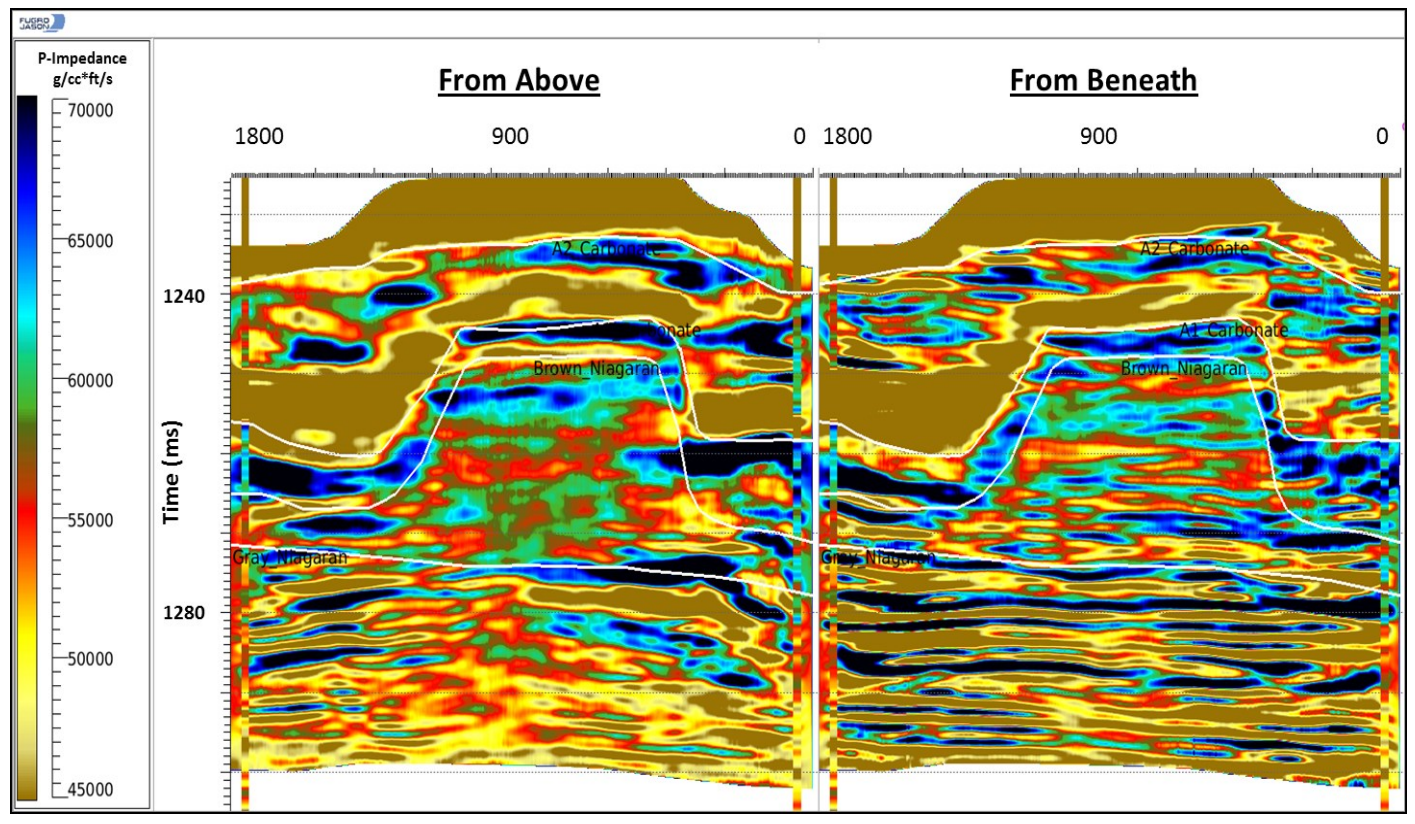

Figure 3.11: "Apparent" acoustic impedance from inverted Springdale seismic data from above (left) and from beneath (right). The white lines indicate horizons used in guiding the inversion, and show the rough outline of the reef itself. Warmer colors (red, yellow) indicate apparent low impedance, while cooler colors (green, blue) indicate apparent high impedance. Because the data are not normal incidence, the correlation to impedance is not precise, and may have significant errors in places; however, the variations are real. From the inverted data, we can readily identify the reef and observe several interior layers that are fairly continuous across the reef.

The optimal elastic parameters are estimated by minimizing an objective functions which contains multiple terms of misfit functions. These user-control misfit functions are designed to provide a solution that honors the seismic data, keeps the sparsity criterion and constrains the results to known geology (Debeye and van Riel, 1990; Pendrel and Van Riel, 1997; Sam and Saussus, 2008). In general, the misfit functions are of the form of an Lp norm. Each term is normalized by the standard deviation of the samples so they can be used together in one objective function. These misfits are related to each other and tend to act under tension where one term becomes smaller the other terms tend to become larger. The objective function is evaluated after every update of the elastic parameters until either a minimum is reached or the maximum number of iterations is reached.

Simultaneous AVO inversion, in practice, uses multiple volumes of seismic data, well logs, seismic velocities (optionally) and interpreted horizons to generate the volumes of elastic properties (P-impedance, S-impedance or $\mathrm{Vp} / \mathrm{Vs}$ and density). The well control is used to calibrate the scaling of the wavelet from seismic to well log units, and also to restore the low frequency (long-wavelength) component of the profile. 
Thus, one can recover an absolute measurement of impedance throughout the seismic data set. As the name refers, simultaneous inversion attempts to find the elastic properties that fit all partial stacks and obey all the constraints.

The simultaneous inversion from a series of partial stacks can be conducted over the full range of acquisition, or they could be restricted to limited ranges in an effort to avoid complexity from phase rotations beyond critical angles. The simultaneous inversion used in this work is not approximation but solves multiple equations for compressional and shear velocities and density using the Knott-Zoeppritz reflectivity equations. S-impedance as an output of deterministic simultaneous AVO inversion is shown in figure 3.12.

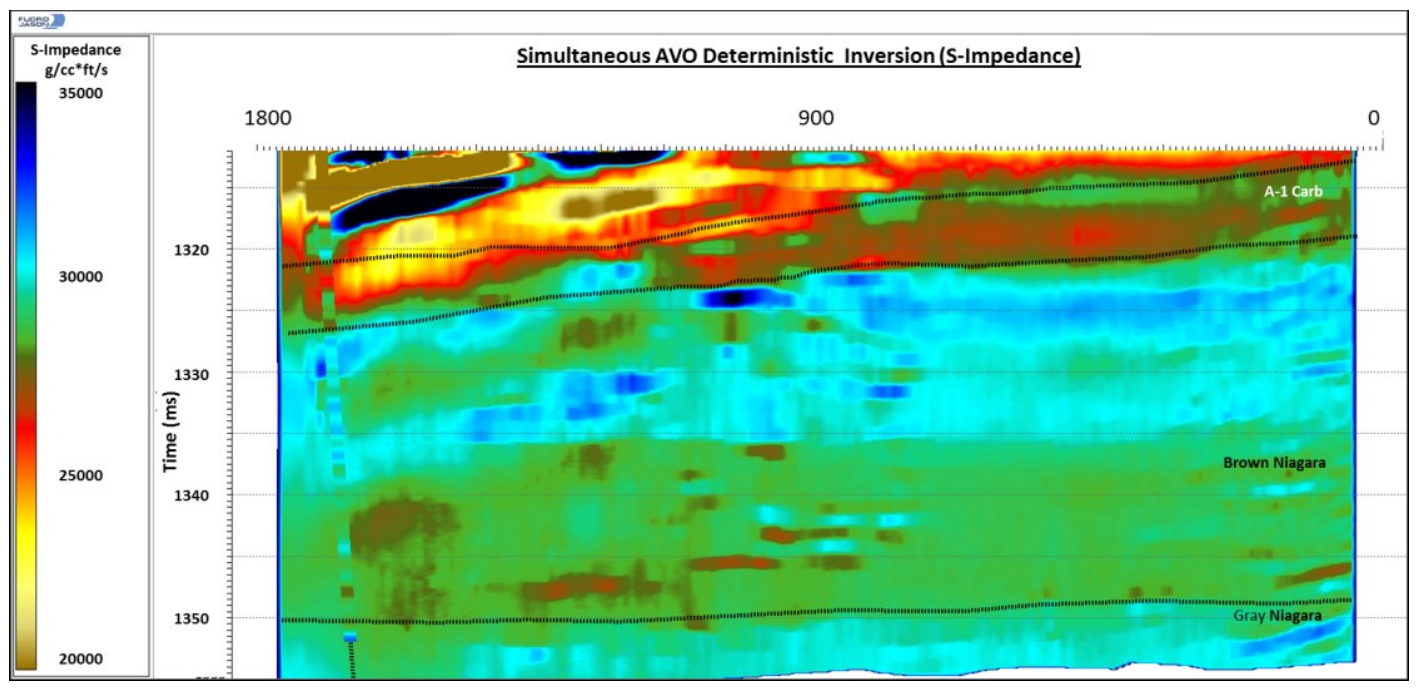

Figure 3.12: S-impedance results from simultaneous AVO inversion for Coldspring. The result was obtained using three angle stacks (total range $30^{\circ}-72^{\circ}$ ).

The goal of reservoir characterization is to draw inferences about the petrophysical properties of the reservoir (porosity, saturation, permeability, etc.) on the basis of geological assumptions of the subsurface, together with evidence from seismic and well $\log$ data. The objective is to capture as much information from each of these various sources as possible, and to leverage synergies between them.

This means interpreting the seismic (which is present everywhere, but provides only indirect and limited-resolution evidence for the petrophysical properties) in the light of a geologically and petrophysically inspired model of the general character to be expected of the subsurface. Since some uncertainties will incorporated about what's down there, the one needs to ensure that there is a good idea of the kinds of answers that are and are not consistent with all the data, and how likely they are. Geostatistical inversion was developed specifically to tackle such issues. 
Geostatistical simultaneous AVO inversion brings together aspects of Bayesian inference, geostatistics and classic AVO inversion within a Markov Chain Monte Carlo (MCMC) framework. The method can incorporate well information formally or run in a blind-to-the-wells mode. Another fundamental feature of the geostatistical method is the possibility of imbedding facies or lithotype (facies) estimation formally within the inversion procedure. The output attributes of each voxel are then, Pimpedance, S-impedance (or $\mathrm{Vp} / \mathrm{Vs}$ ), density and facies. In addition, any other reservoir properties (eg. Porosity, Permeability, Sw), can be estimated simultaneously within the inversion procedure or cosimulated in a follow-up runs.

The geostatistical method recognizes no single answer but rather a set of solutions, all belonging to a joint probability density function (pdf) determined by the method of Bayesian Inference. Bayesian Inference is a probabilistic inference method for systematically incorporating various sources of uncertain information (beliefs) together in an unbiased and consistent manner. These beliefs can take a variety of forms including variogram ranges, lithotype probabilities, multivariate probability density functions (pdfs), wavelets and seismic angle stacks. Taken individually these beliefs do not provide enough information to generate highly detailed and realistic model of the reservoir (Jarvis et al., 2008), but together can provide constraint on the rock properties of interest.

The Markov Chain Monte Carlo (MCMC) technique samples the joint pdfs (usually relations between velocities and density to each other and to and other rock properties) to find a fair set of possible outcomes, each consistent with the input seismic data. From the variability of these outcomes (simulations or realizations) uncertainties and probabilities of the interpretations can be inferred. Because the algorithm works on a (multi-channel) voxel-by-voxel basis rather than trace-by-trace, remarkably few realizations are required to estimate probabilities. Usually, 10 to 20 are sufficient.

The benefits of using MCMC are gained by deriving analytical model that accurately depicts the character of the subsurface. The model accounts both for geostatistical continuity, and for lithology with the consequent discontinuities and multimodalities. With MCMC this analytical model is combined directly with a forward model for the seismic. Given the nonlinearity of these two models, the search for solutions compatible with the resulting posterior PDF must be performed in an iterative manner, using gradual updates to home in on a good realization. More specifically, Markov Chain Monte Carlo will give a statistically fair sample of realizations. 
In practice, the first step in geostatistical inversion using Markov Chain Monte Carlo is by creating a 3D grid that follows the interpreted stratigraphy of the structural model. Then, we discretize the continuous elastic and petrophysical properties of interest on this 3D grid. On the same 3D grid, we also define a discrete property representing lithology logs. We create this lithology logs (or lithotypes) from well logs by assigning appropriate cutoffs that is suitable for the reservoir and the objectives. We express all available evidence and assumptions as a series of probability density functions (PDFs) defined over the property volumes. These evidence and assumptions include; variograms, multivariate joint PDFs, seismic, well logs and any other available information such as fluid contacts information and fluid substitution effects. We combine the PDFs arising from the various data sources described above to yield a Bayesian posterior distribution over the $3 \mathrm{D}$ reservoir property and lithology volumes. Combining these PDFs is a simple matter of applying Bayesian inference principles. The advantage of having this global or ensemble posterior PDF is that it is a true multivariate PDF and thus fully describes the statistical dependencies of all scales - from the micro scale on a few micro layers to the macro scale on a group of traces. We then use a customized Markov Chain Monte Carlo algorithm to obtain a fair sample of realizations from the PDF. A realization consists of a $3 \mathrm{D}$ image of each reservoir property, and of lithology, discretized on a high resolution stratigraphic grid. No single realization is guaranteed to be a perfect match to what we are trying model, but between them they give a good, unbiased idea of what is known from the combined data, as well as what isn't known.

Geostatistical inversion produces results with far more detail than deterministic inversion (Figure 3.13); at well locations, they are highly accurate, and decrease in reliability away from wells. Geostatistical inversion models the lithology and the elastic properties jointly, instead of consecutively as in deterministic inversion, thus leveraging synergies. The magnitude of difference in details between both types of inversion becomes increasingly pronounced for thinner reservoirs, particularly those beyond the vertical resolution of the seismic (Sams and Saussus, 2008). 


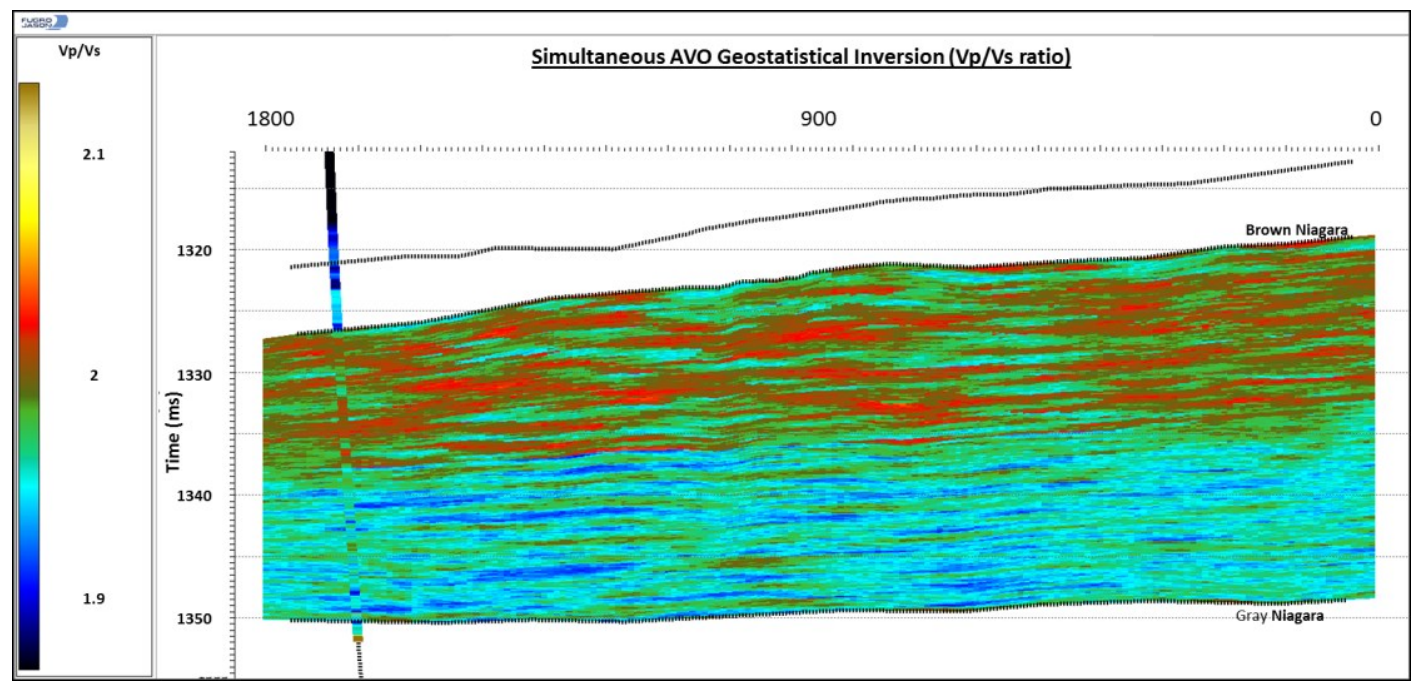

Figure 3.13: Vp/Vs ratio geostatistical inversion result for Coldspring. The result shows higher detail inside the reef compared to the deterministic inversion result.

In the case of carbonate reservoirs under production, there are two major features that we should try to image: porosity and free secondary or injected gas. Both of these will be indicated more strongly in the density term; $\mathrm{Vp}$ is determined by porosity and, to a lesser extent, fluid content; whereas Vs will be almost exclusively determined by porosity rather than fluid content. Seismic inversion should be able to provide stable and good results for $\mathrm{Vp}$ and $\mathrm{Vs}$ even with using a minimum number of independent angle datasets needed to run the inversion (3 partial stacks). In order to obtain density, the recommended number of partial stacks is based on a compromise of stability of inversion parameters, signal-to-noise ratio, and wavelets. If the density term is an objective of the inversion, we would like to use as many partial stacks as possible; however, the number of stack that can be created out of the gathers is dependent on the signal-to-noise ratio and the quality of the seismic data itself. It is often very difficult to obtain a good, reliable density result, even with enough angle range, because of limitations due to the quality of data.

P-impedance is most tightly coupled to porosity (figure 3.14), and is a key to reservoir characterization. The conventional approach for generating porosity distribution from impedance is by setting up a correlation between the porosity and pimpedance from well logs. The geostatistical inversion should give a better porosity compared to the results driven from deterministic inversion. 


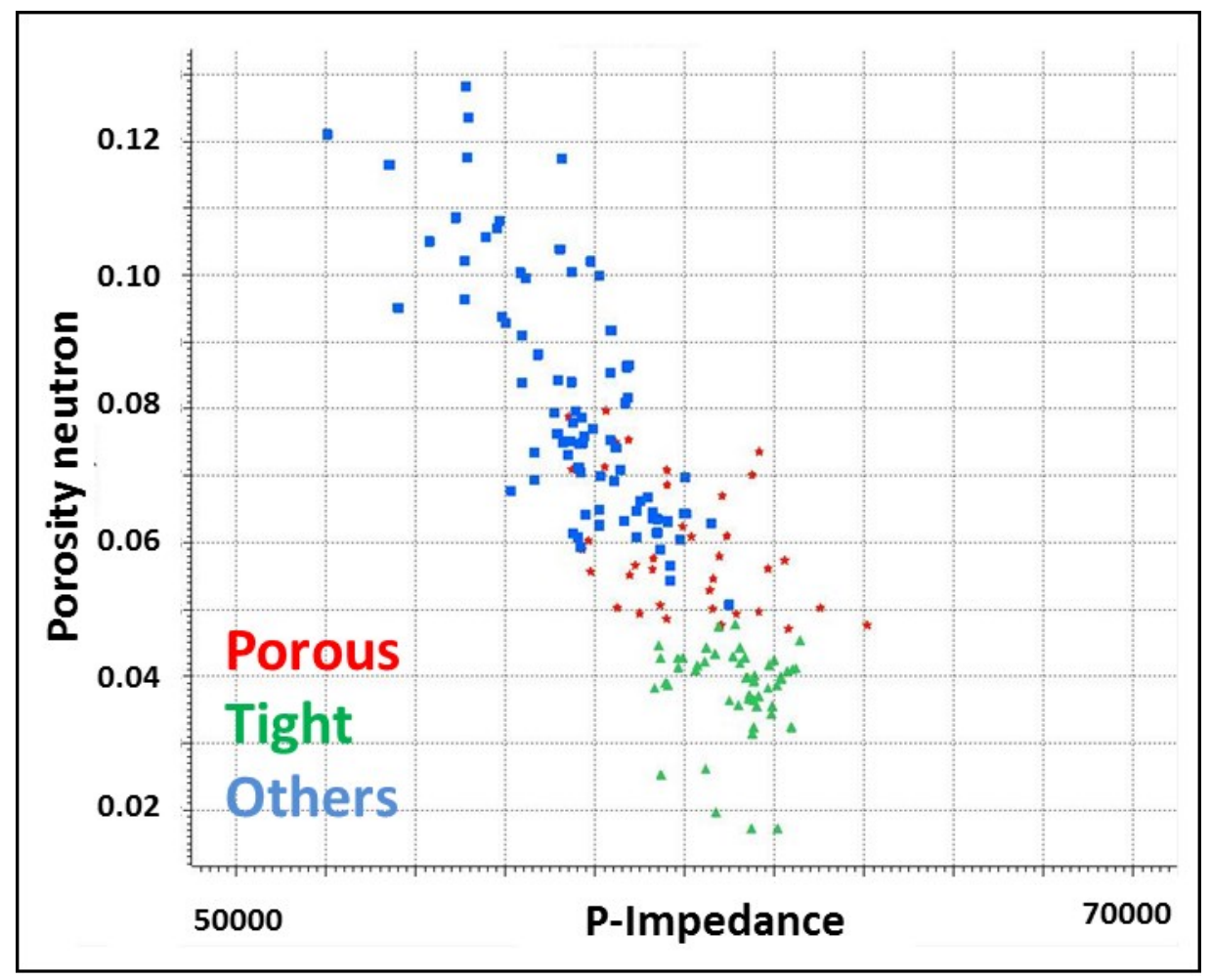

Figure 3.14: A well log cross plot shows the linear relation between the P-impedance and porosity. Lithology type is indicated by color (porous, tight and others). 


\section{Crosswell Seismic Imaging of Acoustic and Shear Impedance in a Michigan Reef}

Acoustic and Shear Impedance images, obtained from deterministic simultaneous inversion of a high-resolution crosswell seismic survey was used to obtain the internal structure of Niagaran reef in Michigan. The crosswell seismic survey was conducted using two monitor wells located external to the reef. These wells extended depths greater than the reef, and imaging was conducted using reflections both from above and from beneath the reef resulting in the best seismic images of any Niagaran pinnacle reef obtained to date. The top of the reservoir can be clearly distinguished, as well as its lateral extent or dipping edges. Reflection events internal to the reef are evident; some of them are fairly continuous across the reef and others are discontinuous.

The wide angles used in crosswell imaging result in amplitude-versus-offset character that is not usually observed in surface data. The data include reflections from angles that range beyond critical for many of the interfaces, and some reflections are visible only for a small range of angles, presumably near their critical angle. Inversion of the seismic data increased the details of the internal structure of the reef and showed a distribution of internal layers that is reasonable for this reef. The Shear-Impedance results were more sensitive in this carbonate environment and seem to correspond to variations in porosity within the reef structure, as well as anhydrite plugging. Signal attenuation appears to be strong when seismic ray paths pass through the upper part of the reservoir; this may be due to intrinsic attenuation and/or scattering of events due to the locally strongly varying gas saturation and extremely low fluid pressures. The seismic images obtained, and interpretations of them, as assisted by inversion, provide insight into the internal geometry of this reef and match the geologic interpretation of the reef model quite well. The results provide data that should be useful for reservoir management. 


\subsection{Introduction}

Surface seismic observations have developed a large set of auxiliary observations that provide a robust evaluation of reservoir properties but this technology still limited in resolution by many factors. Crosswell seismic data can be used to improve significantly resolution of both the vertical and lateral heterogeneity of the carbonate reservoirs, as pointed out by several investigators (Lazaratos, et al., 1991; Lazaratos, 1993; Rector et al., 1995) due to the closer location of sources and receivers to the reflectors, and to the higher frequencies that this allows.

The wide angles present in crosswell imaging provide additional information not present in typical surface-seismic acquisition, and often includes the critical angle. In some instances, the critical angle may provide the only range in which a reflection can be observed above background noise, and inclusion of these angles in a final stacked image can be very beneficial (Trisch, 2006; Pennington et al., 2008). Beyond the critical angle, severe phase rotation of the wavelet occurs, and the stack can be degraded. Therefore, for most detailed amplitude-versus-angle (AVA) studies, a restriction to narrow angles may be prudent.

Inversion involves converting the seismic data into formation properties by integrating the reflection coefficients that are in turn derived from the reflection amplitudes (Tarantola, 1984 \& 1986; Debeye and van Riel, 1990; Pendrel and Van Riel, 1997). Through inversion, one can generate an image of acoustic impedance for normal-incidence reflections. In addition, this technique can be extended, through the use of elastic impedance, to non-normal-incidence seismic data. One can create partial stacks from different angle ranges and invert them, solving simultaneously for an earth model in which the three elastic properties (compressional and shear impedances plus density) are consistent across all angle ranges. Because the crosswell data have angles that are far from normal incidence, the results will present a case not previously encountered in the literature on inversion and interpretation.

The target reef, in Springdale field, is part of the Niagaran (Silurian) reef trend located in the northern part of the Michigan basin. These reefs have been important hydrocarbon producers with a cumulative production of 475 MMBO (76 x $106 \mathrm{~m} 3)$ of oil and 2.8 TCF (79 x $109 \mathrm{~m} 3$ ) of gas (Wylie and Wood, 2005). The crosswell data set was recorded from dedicated monitor wells located outside of the reef at $2000 \mathrm{ft}$ $(600 \mathrm{~m})$ interwell distance. The survey was designed to have shots and receivers at depths ranging from much shallower to much deeper than the reef, located at $4700 \mathrm{ft}$ (1425m). A 100-3000 Hz sweep was used, and final stacked images contained 1000

$\mathrm{Hz}$ data. The fluid pressure inside the reef is $25-50 \mathrm{psi}$. Details of the acquisition and initial processing were described by Trisch (2006) and Pennington et al. (2008). 
Oil or gas reservoirs in reefs can be extremely heterogeneous. Geologists model reefs with many internal layers and zones which can provide internal barriers to flow and isolate compartments. The identification of these facies will improve the recovery of oil significantly. Surface seismic typically cannot image these internal facies, but the application of crosswell seismic and inversion techniques will improve the image by $1-2$ orders of magnitude, as seen in the following sections.

\subsection{High-resolution crosswell imaging}

Figure 4.1 shows the raw data from a common-receiver gather and spectrum for a receiver at $5830 \mathrm{ft}$ depth, with source locations ranging (every ten feet) from $4140 \mathrm{ft}$ to $6000 \mathrm{ft}$. The arrivals are clear and distinct, and reflections and converted phases are apparent even in this gather, prior to stacking.

The initial processed image provided by Z-Seis Inc. is shown in Figure 4.2. The acquisition survey allowed reflection imaging in the usual configuration in which sources and receivers are at depths shallower than the reflector, providing an image "from above". The data that were collected, however, also allow imaging "from beneath" (sources and receivers at depths below the reflector). Angles range from about $30^{\circ}$ to near $90^{\circ}$ (Figure 4.3).

Figure 4.4 shows seismic stacks and spectra for the crosswell data from above and beneath. The full stacked image for crosswell seismic data includes angles that are beyond critical for many of the interfaces, and some reflections are visible only for a small range of angles, presumably near their critical angle, but stack in nonetheless. In fact, stacking these angles in provides an opportunity for these events to be seen on the stacked image, where otherwise they would have been unrecognized. Even though amplitudes may remain large beyond the critical angle, phase rotation (refer to figure 3.4) of the wavelet results in largely incoherent stacking, and these post-critical angles may not contribute to (nor damage) the stacked image significantly. Interfaces for which a critical angle exists in one direction (e.g., "from above") are those for which there is no critical angle in the other direction ("from below"), and vice-versa. 


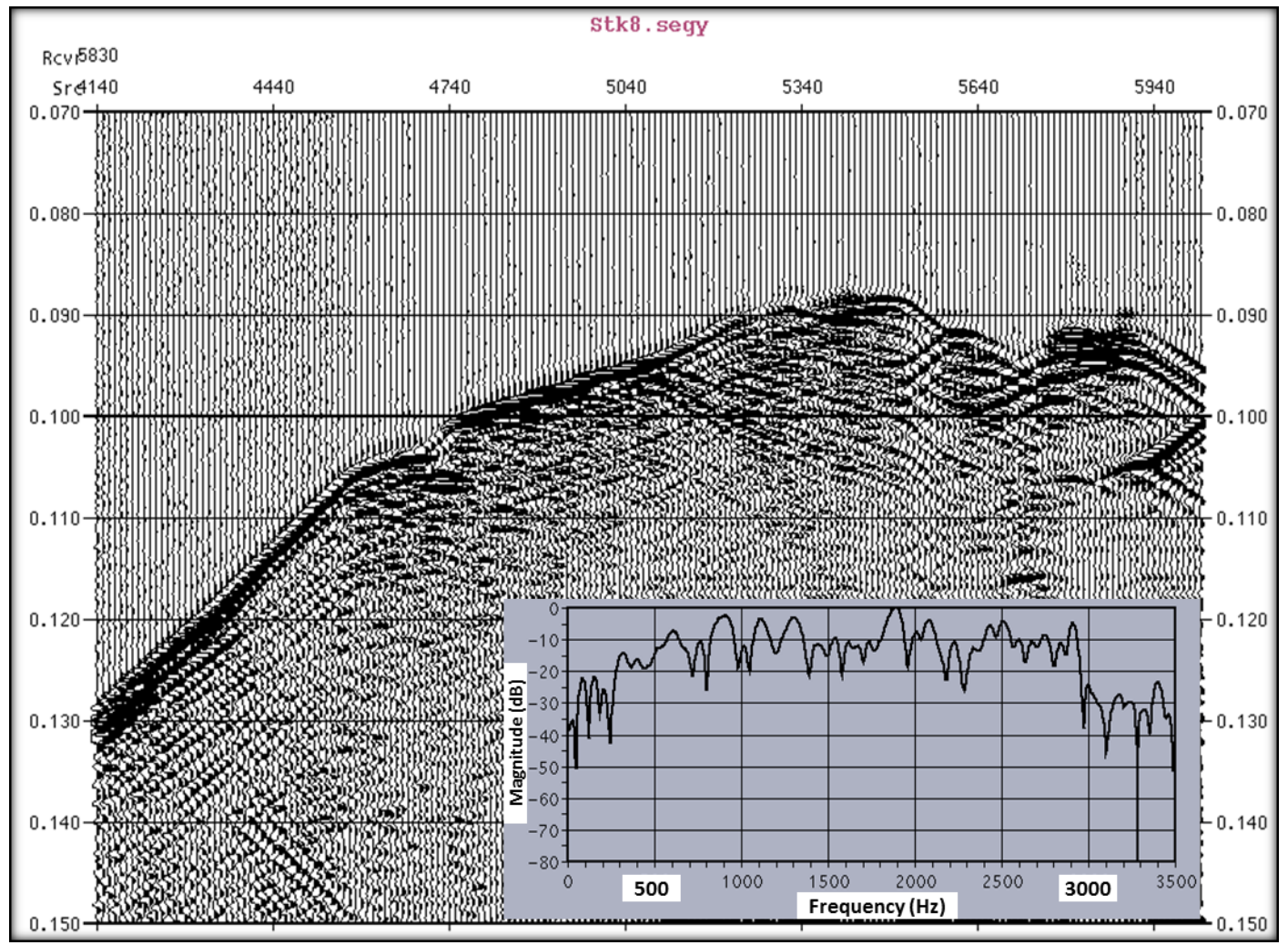

Figure 4.1: A common-receiver gather and its spectrum (courtesy Deep Look CS previously, Z-Seis). 


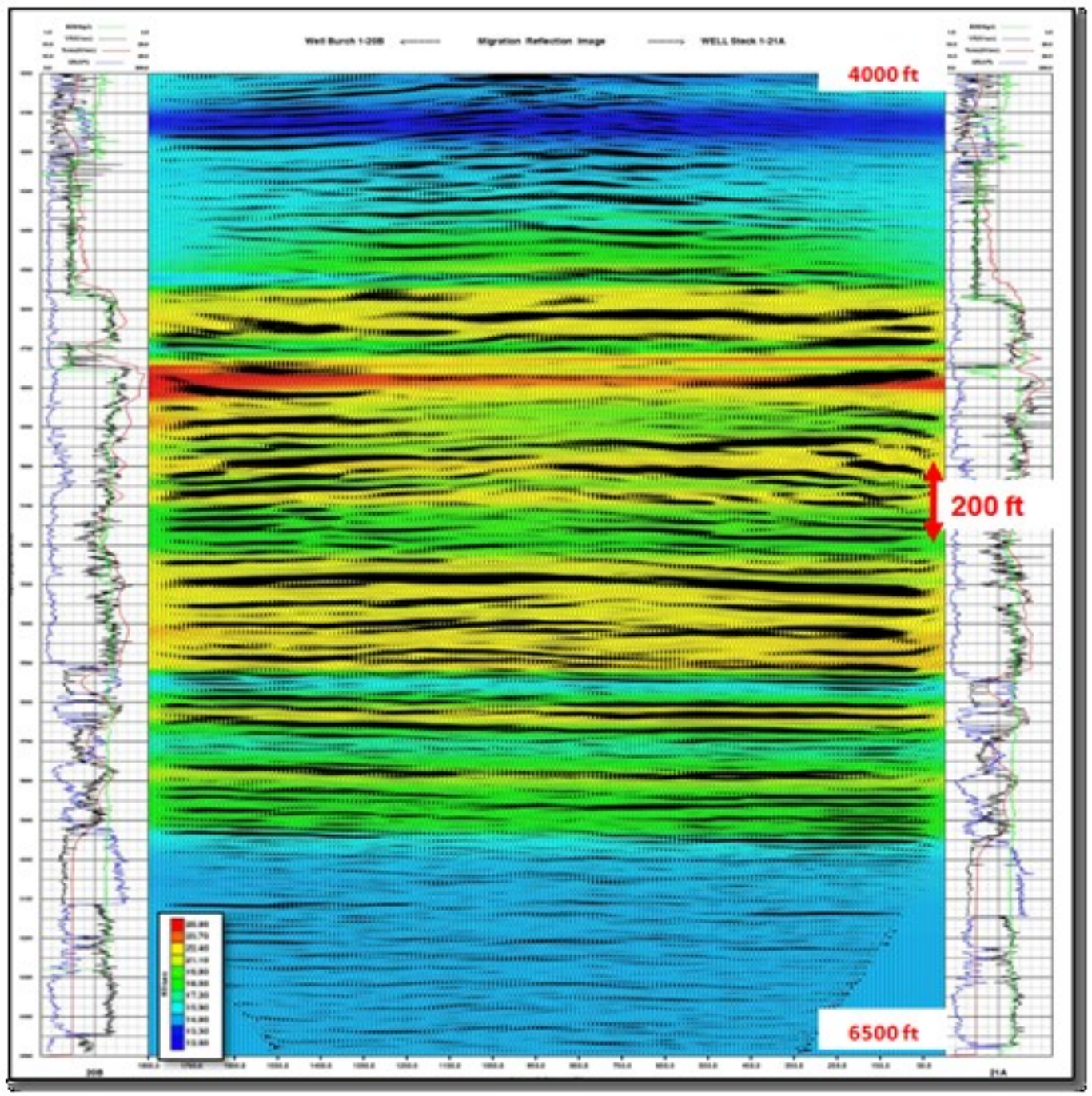

Figure 4.2: Processed seismic crosswell image provided by Z-Seis. Color background represents velocity model (in $\mathrm{kft} / \mathrm{s}$ ) determined by crosswell transmission tomography (errors can be large near the edges of the image). Wiggle traces are the migrated stacked seismic data from crosswell reflection imaging (from above). Various logs are displayed along the edges, at the locations of the Burch (left) and Stech (right) boreholes. Vertical axis is in depth, ranging from 4000 to $6500 \mathrm{ft}$ (1210 to 1970m). Warmer colors (red, yellow) indicate high velocity values. 


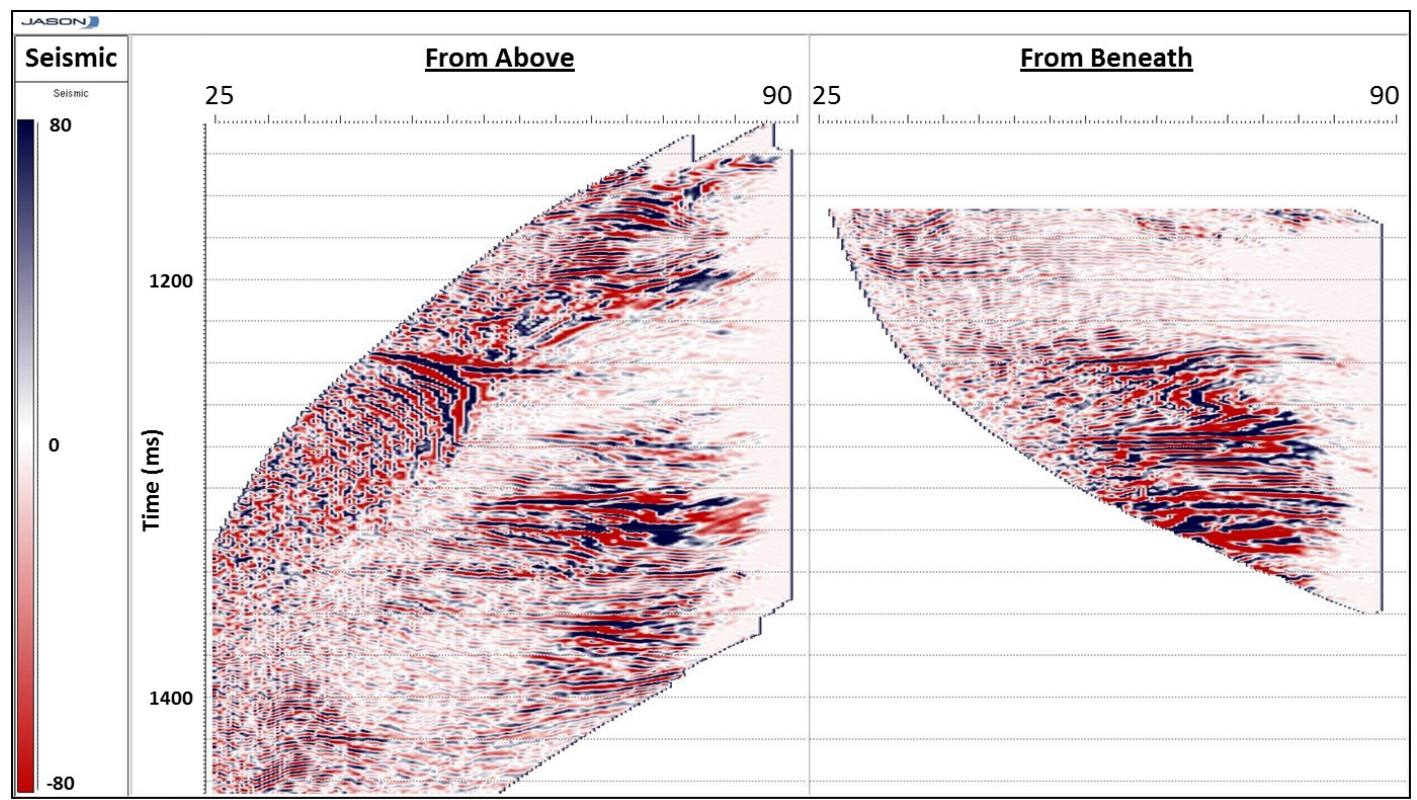

Figure 4.3: Angle gathers at CMP 1000, midway between the wells, from above (left) and from beneath (right). The angles are $25^{\circ}-90^{\circ}$ (from left to right on the horizontal axis). Vertical axis is in time.

The amplitudes of reflectors from within the reef itself are low, especially for the image obtained from above the reflectors. We attribute this to attenuation and scattering of signal as it passes through the complex upper part of the reef, where gas saturation likely varies locally, reservoir pressure is extremely low, and structural complexity of the reef is evident. The image from beneath does not pass through this complexity and only experiences the gas saturation and low pressures at the upper edge of the image, allowing most of the reef image to be higher quality. Thus, in complex environments within hydrocarbon reservoirs, the ability to image from beneath may often be a great advantage. Of course, this involves having deep wells, deeper than the reservoir, in which to locate the sources and receivers. 

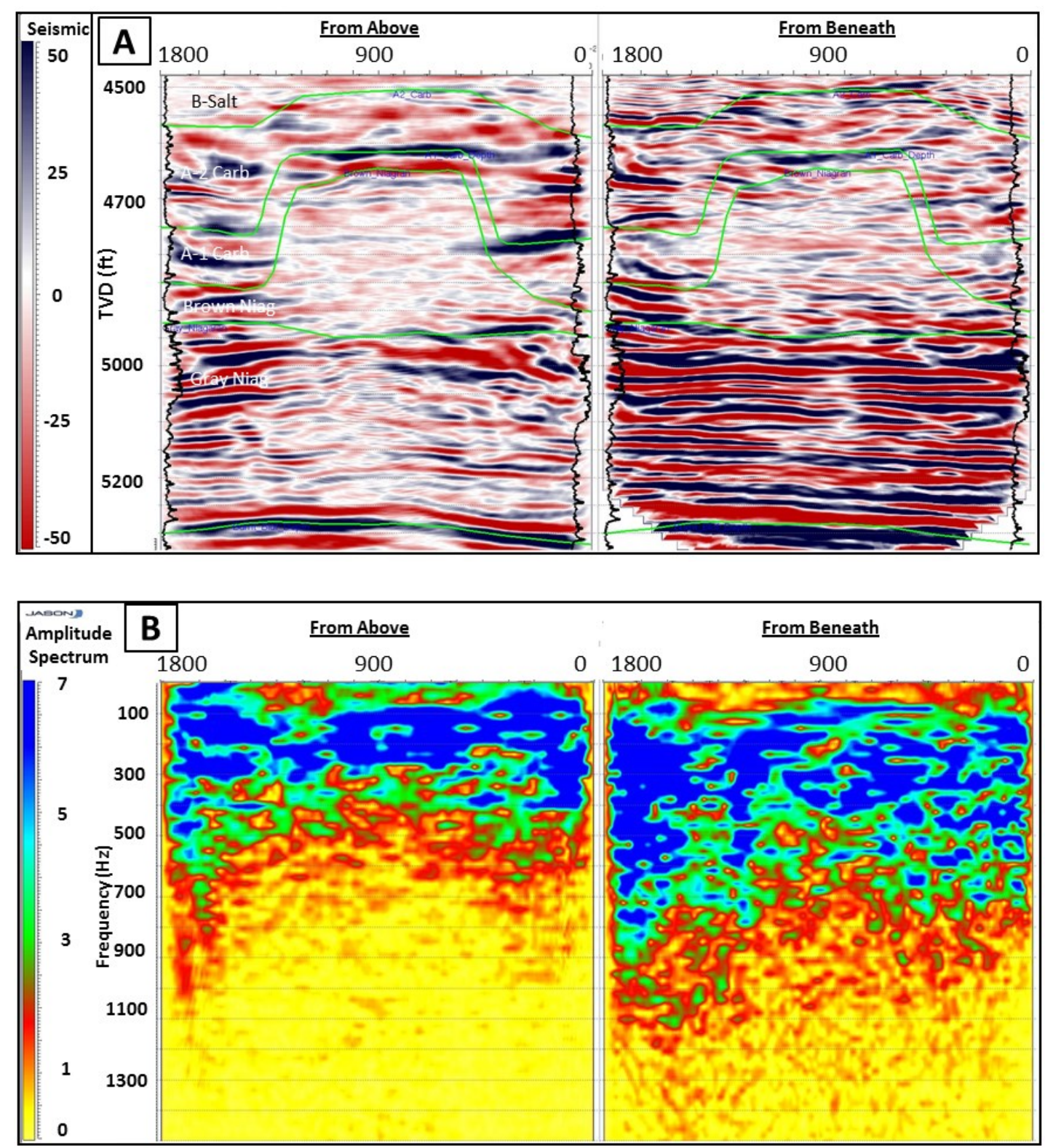

Figure 4.4: a) Stacked image of Springdale crosswell data, from above (left) and from beneath (right) while b) shows the spectra for the same data sets, for a time gate within the reef only. The reef is readily visible as the low-amplitude region at depths of about 4700 to 5000ft near the center of the image. Green lines are seismic horizons. Low-amplitude reflections can be seen within the reef. The image "from below" is more detailed than the one "from above" and is richer in high-frequencies. 


\section{3 "From above" versus "from beneath" CSSI inversion}

The deterministic inversion of partially stacked seismic amplitude data used in this paper is based on constrained sparse spike inversion (CSSI) algorithm used in software provided by Fugro-Jason (Debeye and van Riel, 1990; Pendrel and van Riel, 1997). This inversion algorithm can be used in two ways; full-stack (which is often assumed for surface-collected data to be zero-offset "acoustic"), or based on anglestacks representing limited ranges of angles in each stack (non-zero offset, or "elastic"). Particularly in our crosswell data, the full-stack inversion is, of course, not "acoustic" because of the very-wide angles involved, so the resulting image (Figure 4.5 ) is referred to here as showing "apparent" impedance, without reference to acoustic or elastic response. As long as the angles included in the full stack do not include many critical-angle reflections, the resulting apparent impedance can be used as an indicator of some formation property (or combination of properties); in our case, the formation property indicated is almost entirely porosity, because the seismic properties of the carbonate rocks in the reef are dependent strongly on porosity, but only weakly on fluid content. On the other hand, the elastic inversion from a series of partial stacks can be conducted over the full range of acquisition, or they could be restricted to limited ranges in an effort to avoid complexity from phase rotations beyond critical angles; in either case, we may be able to determine additional properties of the reservoir, or in this instance, make use of that approach which provides the image with best detail. 


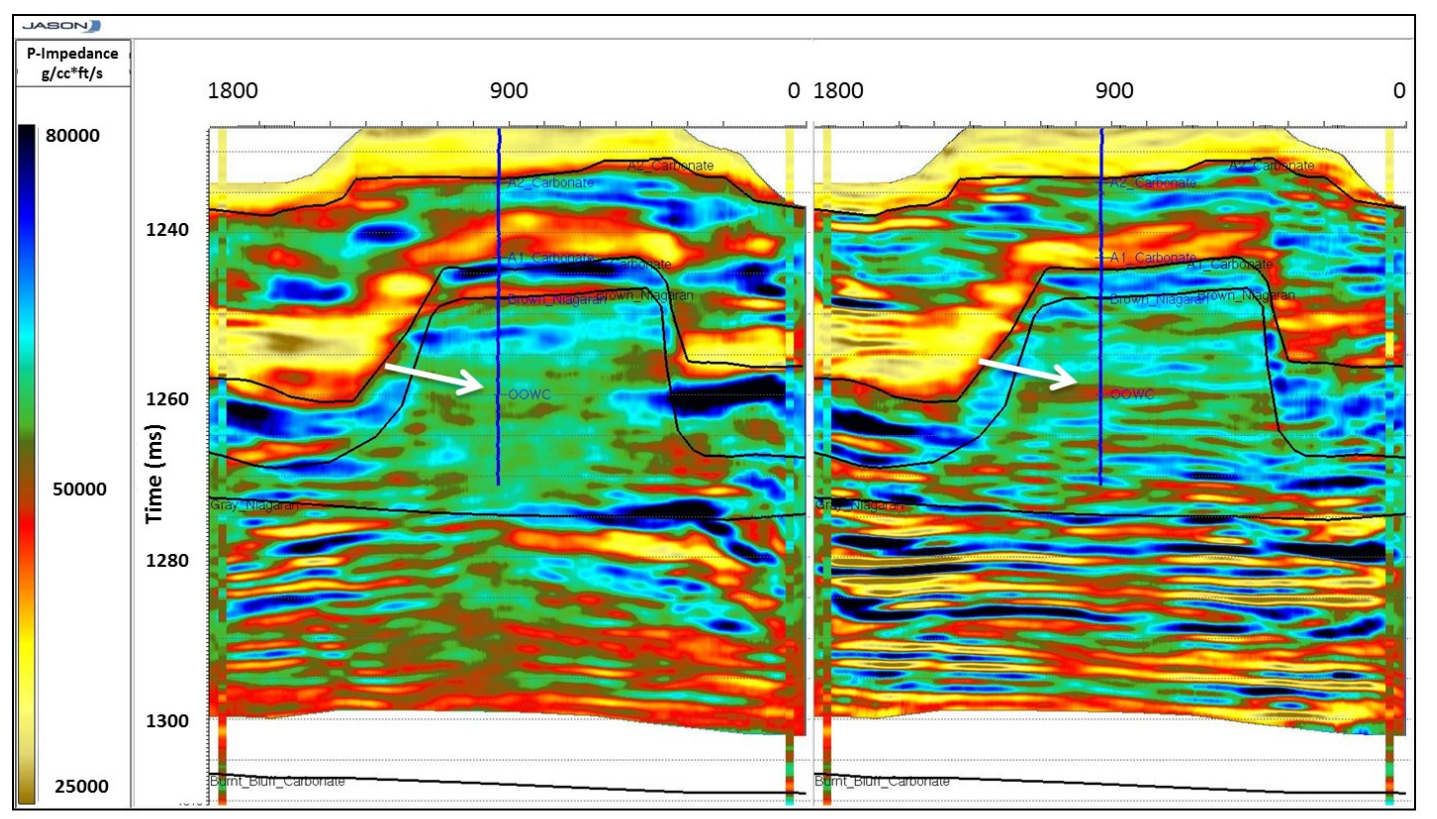

Figure 4.5: "Apparent" impedance from inverted seismic data from above (left) and from beneath (right). Only the area around the reef is shown here. The black lines indicate horizons used in guiding the inversion, and show the rough outline of the reef itself. Warmer colors (red, yellow) indicate apparent low impedance, while cooler colors (green, blue) indicate apparent high impedance. Because the data are not normal incidence, the correlation to impedance is not precise, and may have significant errors in places; however, the variations are real. The blue line in the middle shows the only producing well in this reef, labeled with the original oil-water contact (OOWC, white arrow).

However, when we discuss the "from above" and "from below" processing and images, we should notice a major issue in which the packaged inversion procedures assume that the reflection series is encountered from the top down, and therefore avoid a pitfall in assuming that straightforward use of packaged inversion suites can be used on the traces in either case without modification. It is important to recall that although, for zero-offsete data, the reflection observed from above is the same amplitude of the reflection observed from below, but with opposite sign. Yet, for all non-zero angles, the amplitude will vary strongly, depending on which layer is encountered before the interface, and which after (figure 4.6). So a simple sign change, at any angles other than normal incidence, is not sufficient. Our approach to solve this issue was by "flipping" the "from-beneath" seismic traces (top-down, or last-sample to first-sample), then perform the inversions using standard software, and then "flip" the resulting image back so that the shallow depths are again on top. Figures 4.7 and 4.8 show the elastic inversion results for P- and S-impedances from above and from beneath. 

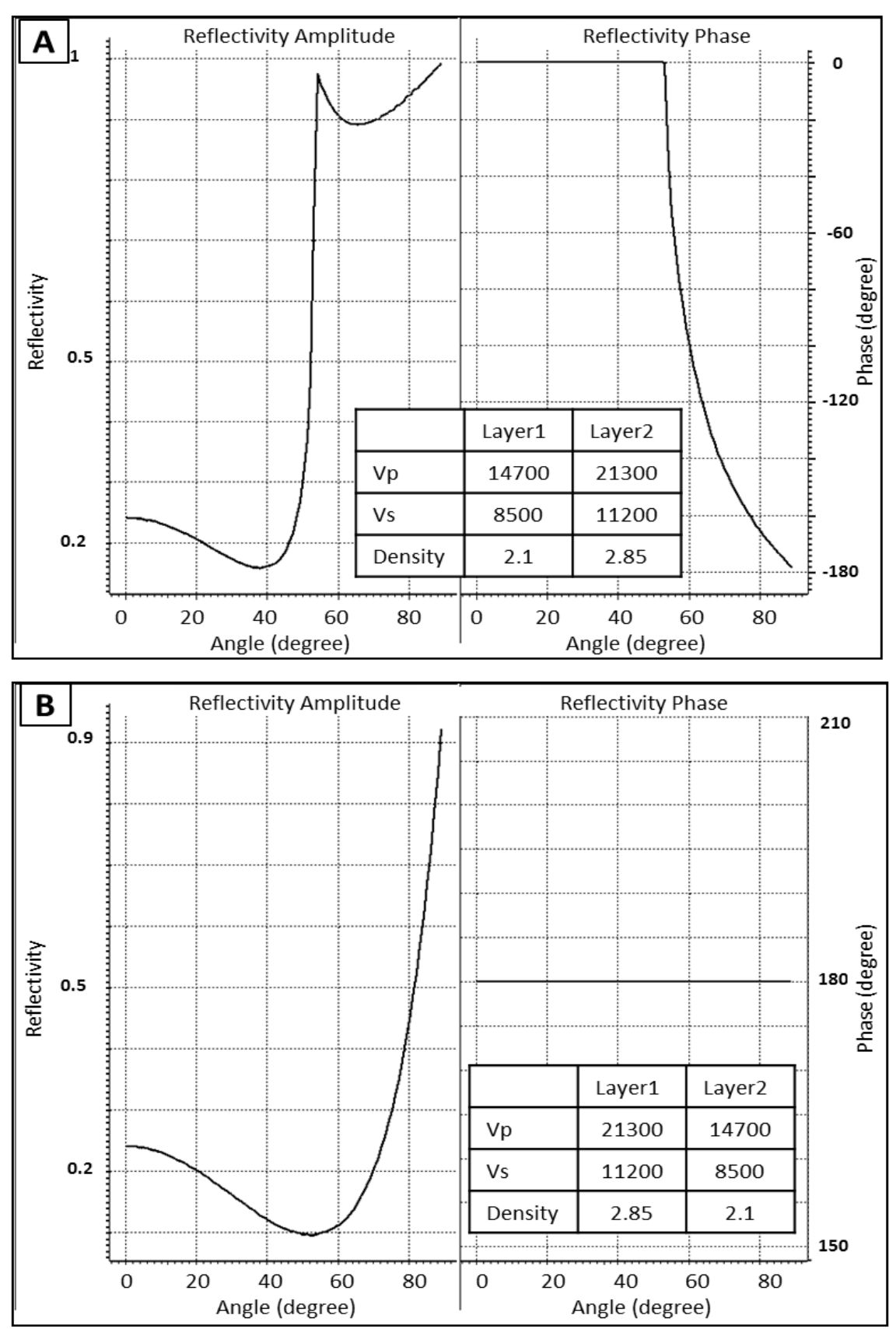

Figure 4.6: Zoeppritz solutions for a two layers model from above (a) and from beneath (b). P-and S-velocity values are in $\mathrm{ft} / \mathrm{s}$ while density is in $\mathrm{g} / \mathrm{cc}$. Notice the reflection observed from above is not just the inverse of the reflection observed from below in all angles except the near normal incidence. Also, notice the severe phase rotation beyond the critical angle in from above figure. 


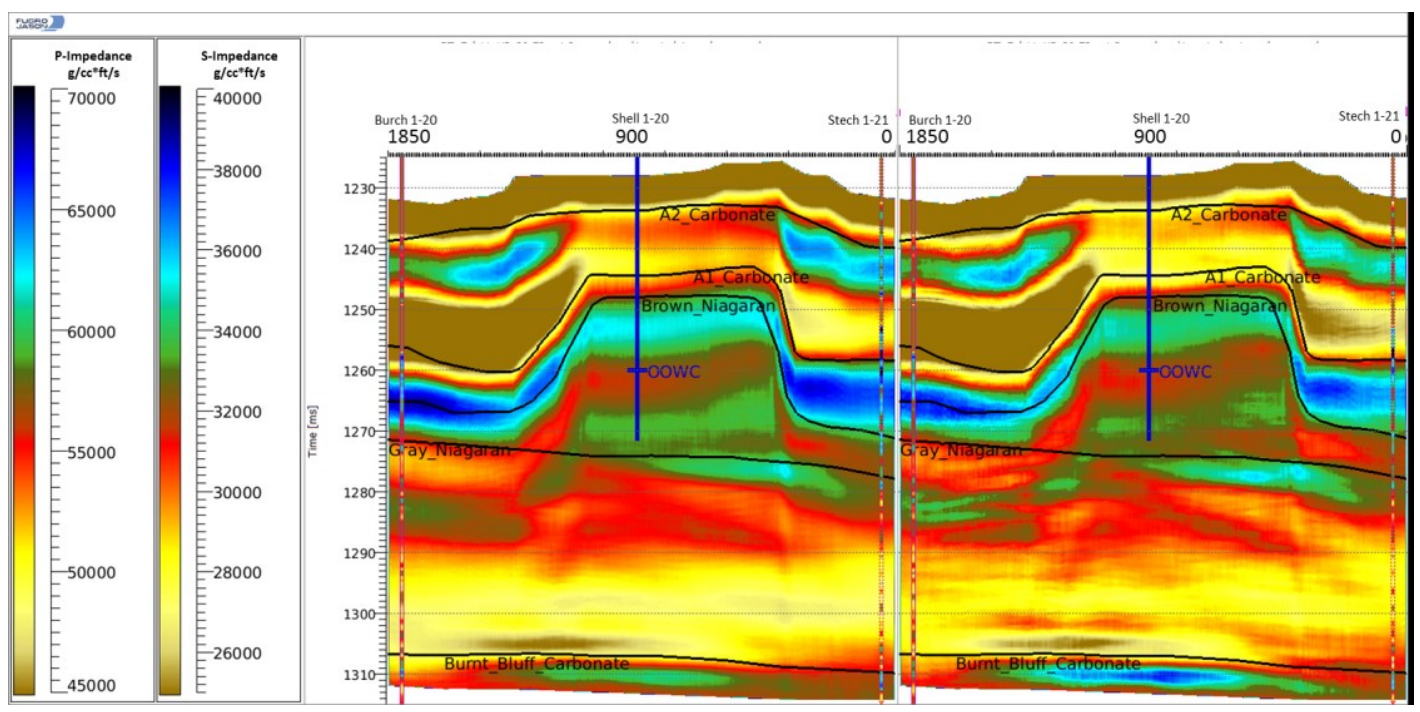

Figure 4.7: Results of elastic inversion from above, using three angle stacks (total range $30^{\circ}-65^{\circ}$ ). These results are expressed in terms of P-Impedance (left) and $S$ Impedance (right).

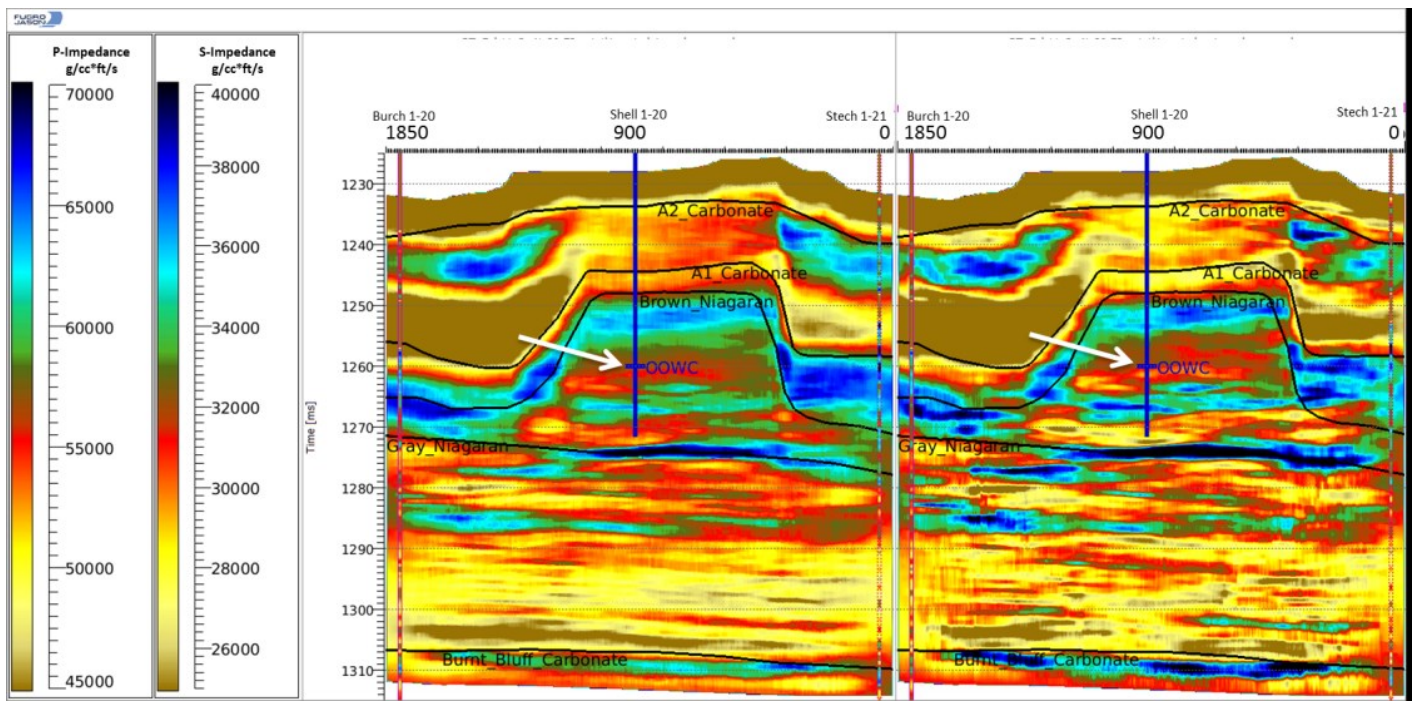

Figure 4.8: Results of elastic inversion from beneath, using three angle stacks (total range $30^{\circ}-72^{\circ}$ ). These results are expressed in terms of P-Impedance (left) and $S$ Impedance (right). The vertical blue line in the middle shows the location of the only producing well in this reef, labeled with the original oil-water contact (OOWC, white arrow). 
The elastic inversion results are products of a simultaneous inversion using the full Zoeppritz equations, and should, therefore, be valid, assuming that our input data contained sufficient breadth of angles to constrain the results; however, we are suspicious of post-critical handling of phase rotations, both in the inversion process and in the initial processing to angle traces. In any case, the results show a distribution of internal layers that is reasonable for this reef. Again, the inversion results "from beneath" (figure 4.8) provide an image of the internal structure of the carbonate reef that appears to be higher resolution than "from above" (figures 4.7). This is consistent with our observations based on the from above and from beneath seismic images. Also, it seems that the shear-impedance results are more sensitive for porosity in such a carbonate environment and indicates which events represent zones of higher porosity (low impedance) and which are lower porosity (higher impedance). This may be a result of a greater sensitivity of shear impedance than acoustic impedance to porosity, or it may indicate that greater complexity that results from (albeit weak) fluid sensitivity of the acoustic impedance, or both.

Imaging from beneath results in apparently higher resolution, showing layers that more continuous than in imaging from above. This is probably related to the fact that the ray paths connecting sources and receivers beneath the reef with their reflection points do not experience the attenuation and/or scattering than those above the reef, either because of the simpler geologic structure at the base of the reef, or because of the fluid and pressure distribution within the upper, produced, portions of the reef. The areas of lower apparent impedance (yellows to reds) suggest higher porosity, while those of higher apparent impedance (greens to blues) suggest lower porosity, with some layers probably having all porosity occluded by anhydrite plugging.

We note that the original oil-water contact in the reef coincides roughly with the lithologic boundary where the lower-porosity reef material (containing the hydrocarbon reservoir) overlies higher-porosity reef material, typical of Michigan reefs. After thirty years of production the fluid contact is no longer expected at this location, and we do not presume to image it. 


\subsection{Reef interpretation}

The upper part of the reef appears to be an attenuating zone in both imaging geometries (from above and from beneath); this supports the notion that it is a property of the reef at this location that causes the increased signal attenuation, probably varying gas saturation and/or fluid pressures that are causing intrinsic attenuation or that are resulting in high density of scatters rendering the signal less coherent. In any case, the image "from above" is not as high-resolution as the one "from beneath".

Amplitudes are easily contaminated by many factors such as scattering, geometric spreading, source and receiver coupling, radiation patterns, and transmission/reflection effects. For scattering and intrinsic attenuation, however, the high-frequency components of the seismic signal are attenuated more rapidly than the low-frequency components. Carrillo et al (2007) calculated the attenuation coefficient for the same dataset (from above) by using the analysis of traveltimes and average frequencies for transmitted rays. Figure 4.9a shows the "apparent" elastic inversion for the crosswell data from beneath. The low impedances (the higher porosity zone) shown at the base of the reef match the high attenuation zone estimated by Carrillo et al. More internal details are shown in the inversion where this attenuation zone has a variation in porosity.

Figure 4.10 shows the shear impedance produced by the elastic inversion of partial stacks imaged from beneath compared with one of the geological models by Gill (1973). His model was based on many core samples had been taken from Michigan reefs. We can use the inversion results to divide the reef into three zones as suggested by Gill. It is apparent that the low-impedance area on the inversion results shown inside the organic reef stage correspond to the reef detritus zone in Gill's model. A similar conclusion can be verified for details within the biohermal stage. Since the shear impedance is more dependent on the variation in porosity rather than the fluid distribution, it seems that shear-impedance is highlighting the internal lithologic features of the reef quite well. 


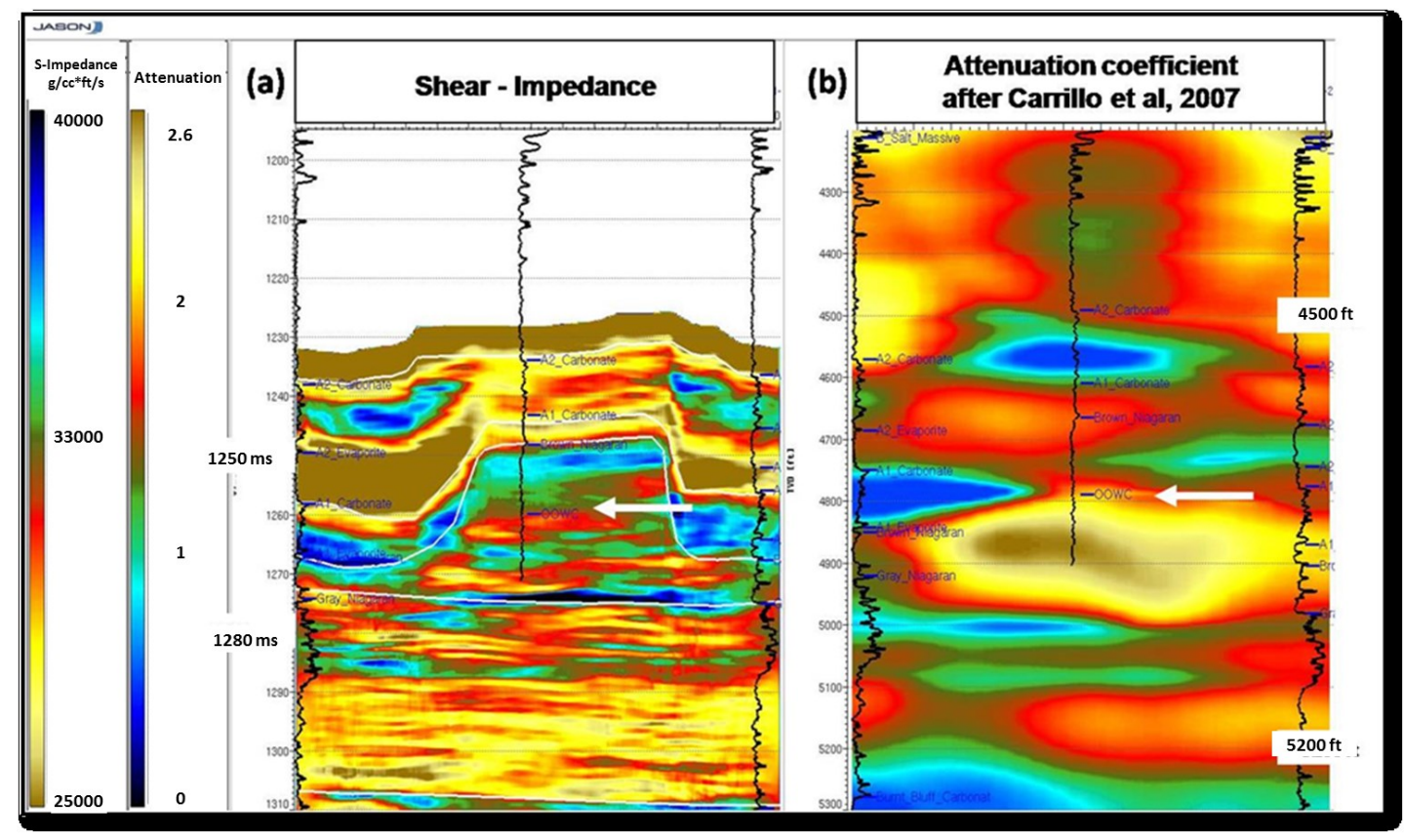

Figure 4.9: a) Elastic inversion (S-impedance, same as 4.8) imaged from beneath. The reef horizons are in white. Vertical scale is in time (ms); b) Attenuation coefficient calculated by Carrillo et al (2007, modified). Vertical scale is in depth (ft). Note that the two images do not exactly correspond because of the differences in vertical scale, between time (a) and depth (b). Black logs are for Gamma-ray and the picked formation tops are shown in blue. Notice the OOWC (white arrow) as interpreted by the operator, and the coincident location of the top of high porosity and high attenuation within the reef. High porosity, inferred from low impedance, is red/yellow in (a), and high attenuation is yellow/gold in (b) 


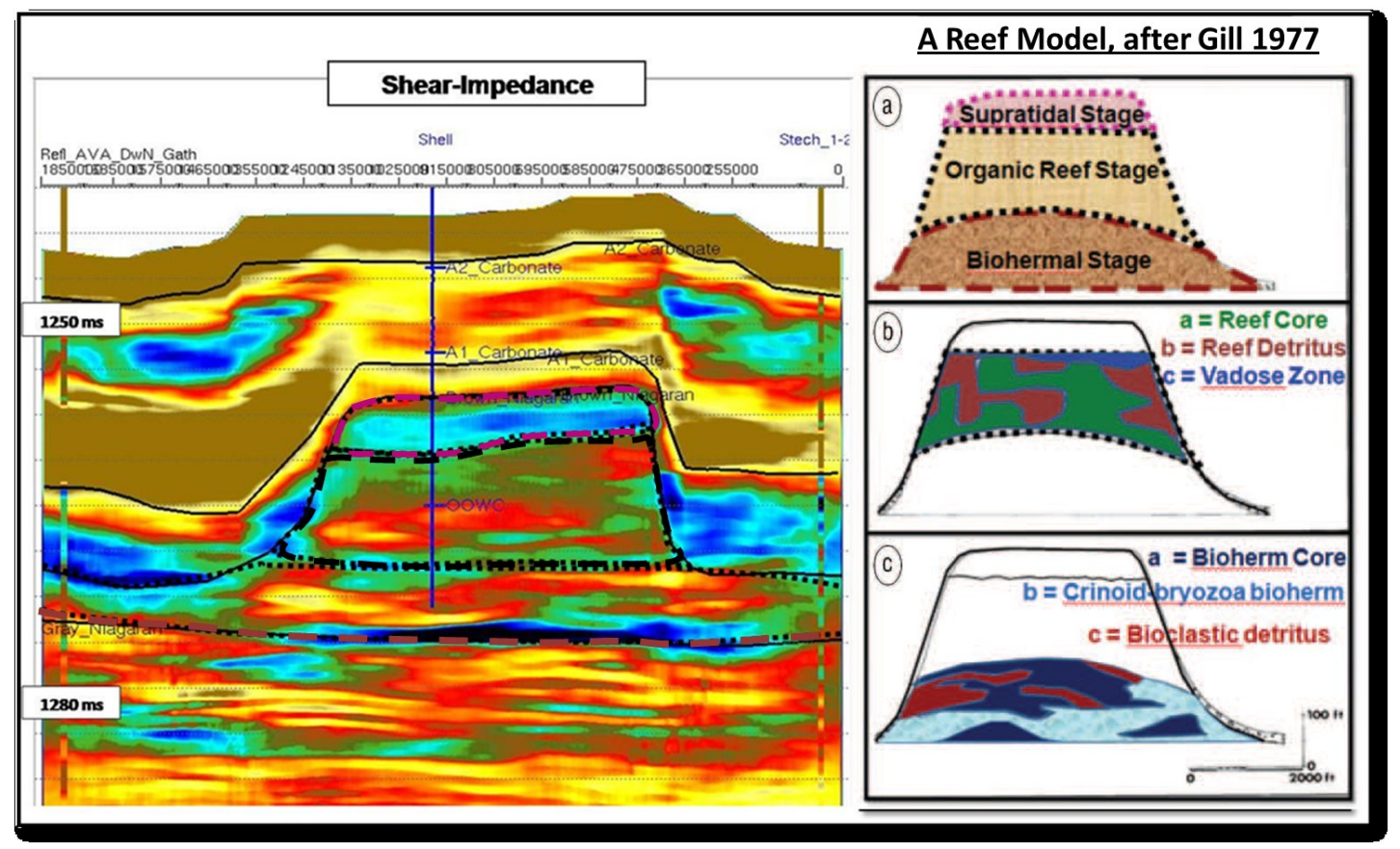

Figure 4.10: (left) Elastic inversion (S-impedance, same as 4.8) imaged from beneath. The reef horizons are in solid black. Color scale is the same as Figure 4.9a, with low impedances (high porosity) in yellow to red, and high impedances (low porosity) in green to blue. The geological reef assessment zones are indicated by dashed black lines. This assessment (interpretation) is based on the reef model after Gill, 1977 (right). Vertical scale is in time (ms). Notice the low impedances shown inside the organic reef stage on the shear inversion results match the reef detritus zone in Gill's model. Also, the same conclusion is verified for the biohermal stage. Even more internal details are shown in the inversion where these zones have a variation in porosity. 


\subsection{Conclusion}

Crosswell seismic imaging has demonstrated its ability to provide extremely highquality images of reservoirs such that internal features within the reef can be imaged and characterized. Resolution is about 40 times that of surface seismic in the same area $(50 \mathrm{~Hz}$ versus $2000 \mathrm{~Hz})$.

The amplitudes of reflectors from within the Springdale reef are low, however, and especially so for the image obtained from above. We attribute this to attenuation and scattering of signal as it passes through the complex upper part of the reef, where gas saturation likely varies locally, reservoir pressure is extremely low, and structural complexity of the reef is evident. The image from beneath does not pass through this complexity and only experiences the gas saturation and low pressures at the upper edge of the image, allowing most of the image to be higher quality.

Until techniques are developed that properly account for crosswell seismic inversion from beneath, we treat the two (above and beneath) data sets independently, rather than providing additional constraints on the simultaneous inversion. Recall that the reflection observed from above is not just the inverse of the reflection observed from below (with a simple sign change), at any angles other than normal incidence.

The most valuable aspect of the reservoir characterization in this study has been the inversion of data. But, because inversion techniques have not yet been developed that properly account for phase rotations beyond critical angle, it is probably most appropriate to restrict inversion to stacks that do not exceed that angle. Within this limited range, elastic inversion may be conducted with reasonable results, as demonstrated at the Springdale site. Although "acoustic" inversion also provides meaningful results, the interpreter must recognize that the resulting image is not truly "acoustic" impedance, but some sort of narrow-angle "elastic" impedance, referred to in this report as "apparent" impedance. Nonetheless, at these angle ranges and in this lithologic environment, a decrease in apparent impedance can be interpreted as an increase in porosity within the reef, while an increase in apparent impedance can be interpreted as a decrease in porosity, perhaps anhydrite-plugged at the extreme, within the reef.

Shear-impedances seem to highlight the internal features of the reef best and the resultant inverted images of pinnacle Niagaran reef are the best images available to date. 


\subsection{References}

Carrillo P., Milagrosa A., Bryans B., and Turpening R., 2007, Attenuation coefficient tomogram and Q distribution image from crosswell survey in the Northern Reef Trend of Michigan Basin; SEG Expanded Abstracts 26, 1252.

Debeye, H.W. J., and P. van Riel, 1990, Lp-norm deconvolution: Geophysical Prospecting, 38, 381-404.

Gill, D., 1973, Stratigraphy, facies, evolution and diagenesis of productive Niagaran Guelph reefs and Cayugan sabkha deposits, the Belle River Mills gas field, Michigan basin: Ph.D. Dissertation, University of Michigan, Ann Arbor, 275 p.

Gill, D., 1977, The Belle River Mills gas field: Productive Niagaran reefs encased by sabkha deposits, Michigan basin: Michigan Basin Geological Society Special Paper 2, $188 \mathrm{p}$.

Lazaratos. S. K.,1993, Cross-well reflection imaging: Ph.D. Thesis, Stanford University.

Lazaratos, S. K., Rector, J. W., Harris, J. M., 1991, High resolution cross-well reflection imaging: 61st Annual International Meeting, SEG, Expanded Abstracts, $350-353$.

Pendrel, J., and Van Riel P., 1997, Methodology for seismic inversion and modeling: A western Canadian reef example: CSEG Recorder, 22, 5-15.

Pennington W. D., Ibrahim, M., Turpening, R.M., Trisch, S.P., Richardson, J.P., Asiala, C., and Mabrouk, W. (2008), Crosswell Seismic Amplitude-Versus-Offset for Detailed Imaging of Facies and Fluid Distribution within Carbonate Oil Reservoirs, report to Department of Energy, available at www.osti.gov/servlets/purl/946424$\underline{\mathrm{Mm} 7 \mathrm{X} 2 \mathrm{f} /}$

Trisch, S.P. 2006, Crosswell Seismic Amplitude Variation with Angle Studies at a Niagaran Reef: MS Thesis, Michigan Technological University.

Rector J.W., Lazaratos S. K., Harris J. M. and Schaacks M.V., 1995, High-resolution crosswell imaging of a west Texas carbonate reservoir: Part 3-Wavefield separation of reflections: Geophysics, 60, P.692-701

Wyllie, A.S., and Wood, J.R., 2005, Well-log tomography and 3-D imaging of core and log-curve amplitudes in a Niagaran reef, Belle River Mills field, St. Clair County, Michigan, United States, AAPG Bulletin, v. 89, No. 4, 409-433. 


\section{AVA Analysis and Inversion of Crosswell Seismic Images taken from Above and from Beneath a Michigan Reef}

\subsection{Summary}

Images of elastic properties, obtained from deterministic simultaneous inversion of a high-resolution crosswell seismic survey, were used to obtain the internal structure of Niagaran Michigan reef. The images obtained are the best of any Niagaran pinnacle reef to date.

This crosswell seismic survey used source and receiver boreholes that extended to a greater depth than the target zone. This unique geometry provides images made using reflections from above and reflections from beneath the various reflecting horizons. In addition, the wide angles used in crosswell imaging result in amplitude-versusangle (AVA) features not usually observed in surface data. These features include reflections from angles that are near critical and beyond critical for many of the interfaces; some of these reflections are visible only for a small range of angles, presumably near their critical angle. Combining the results from images obtained from above with those from beneath provides additional information, first by exhibiting ranges of angles that are different for the two images, especially for reflectors at shallow depths, and second, by providing additional constraints on the solutions for Zoeppritz equations.

For most interfaces, the compressional-wave velocity increases with depth, and the data from above therefore encounter critical angles and associated post-critical phase rotations more often than the data from beneath. Geometric constraints imposed by the borehole locations also result in angle-range differences in the images from above and from beneath. On the other hand, the AVA character must result from identical contrasts in elastic properties in the two sets of images, albeit in reverse order.

While there are many ways to handle these data set obtained from above and from beneath, at pre-critical angles a simple solution is to time reverse the seismic traces (top-down, or last-sample to first-sample) then sign reverse the polarity (multiply the amplitudes by -1), and incorporate them with the time-reversed wider-angle seismograms into the inversion process. Because of signal-processing and imaging constraints, we chose to combine small-angle data from above (time and sign reversed) with the wide-angle data from beneath (time-reversed), taking advantage of the better signal in both ranges, and extending the overall angle range available for inversion. This approach seems to work well for layers exhibiting small contrasts in elastic properties 
The results from this combined approach yields a better image than individual inversions of data from above and from beneath. The final image shows detailed distribution of internal layers that is reasonable for Niagaran pinnacle reef and appears to provide the best image of elastic properties to date for any such object.

\subsection{Introduction}

Surface-seismic methods have led to a large set of technologies and observations that provide robust evaluation of reservoir properties, but this technology is limited in resolution by many factors, including resolution and limited angle ranges for elastic inversion. It is widely recognized that crosswell seismic data can significantly improve resolution of both the vertical and lateral heterogeneity of the targets, including the carbonate reservoirs of interest in this study (e.g., Lazaratos, et al., 1991; Lazaratos, 1993; Rector et al., 1995; Ibrahim, et al., 2009, 2010) due to the high signal frequencies and the closer location of sources and receivers to the reflectors. But the wide ranges of angles present in crosswell seismic data have not generally been exploited in quantitative analysis for elastic properties.

A potential advantage of crosswell imaging to be examined in this paper is the information contained in wide-angle reflections that should provide additional information beyond that available in typical surface seismic acquisition; in crosswell imaging, this range often includes the critical angle. In many cases, a small acousticimpedance contrast may mean that the only reflections observed are those from nearcritical angles, and inclusion of these angles in a final stacked image can be beneficial (Trisch, 2006; Pennington et al., 2008; Ibrahim et al., 2010). Beyond the critical angle, severe phase rotation of the wavelet occurs, and the stack can be degraded; therefore, for most detailed amplitude variation with angle (AVA) studies, a restriction to narrow angles may be prudent (Ibrahim et al., $2009 \& 2010$ ).

A high-resolution crosswell seismic survey was conducted using two monitor wells located external to a Silurian (Niagaran) reef, at Springdale field in northern Michigan. This acquisition survey allowed reflection imaging in the usual configuration "from above" (both source and receiver locations are at shallower depths than the reflector), but also allowed imaging "from beneath" (sources and receivers at depths below the reflector). A 100-3000 Hz sweep was used during the acquisition. The final images contained $1000 \mathrm{~Hz}$ data and angles range from about $30^{\circ}$ to near $90^{\circ}$.

Inversion involves converting the seismic data into formation properties by integrating the reflection coefficients that are in turn derived from the reflection amplitudes (Debeye and van Riel, 1990; Pendrel and Van Riel, 1997). Through inversion, one can generate an image of acoustic impedance for normal-incidence 
reflections and/or the technique can be extended, through the use of an elastic impedance concept, where partial stacks of non-normal incidence data can be inverted simultaneously to obtain three elastic properties (compressional and shear impedances plus density).

Because of the geometry of the crosswell data where no angles of normal incidence do exist in the crosswell data, Ibrahim et al., (2009) called the one-stack inversion an "apparent inversion" where the resulting impedance is, of course, not acoustic due to the wide angles involved.

For any interface has strong contrast in the elastic properties, Ibrahim et al., (2010) noticed that the reflection observed from above is not simply the inverse of the reflection observed from beneath at angles other than normal incidence. As a result, they used the constrained sparse spike inversion (CSSI) to invert individually crosswell data "from above" and "from beneath". However, in this article, the technique will be extended to integrate both data sets and invert them together to take the advantage of wider angle range while avoiding critical angles that often occur in the data "from above".

Ray-tracing, as a forward modeling technique, has been widely used in seismology for decades, to calculate seismic travel times, ray-paths, and synthetics. The raytracing algorithm is an iterative process that builds the ray path step-by-step using linear segment increments through a model in which the velocity changes. Thus the ray path is a set of discretized points with appropriate information of incidence angles. Ray-tracing within a reasonable velocity model can be used to determine the angle coverage (minimum and maximum) of any reflector between the source and receiver wells.

Approximations to Zoeppritz solution always assume small contrasts in elastic parameters and small angles of incidence and fail as the critical angle is approached. For crosswell data, using the approximations is not sufficient because of the wide angles involved $\left(30^{\circ}-\right.$ near $\left.90^{\circ}\right)$. 


\subsection{Geology of Michigan Reefs}

The Niagaran (Silurian) reefs are significant hydrocarbon reservoirs in the Michigan Basin. They are divided into two trends or fairways based on locations and reef geometries. Reefs from the Northern trend are aerially limited ranging from $300 \mathrm{ft}$ to over 600 feet in height and laterally covering up to 200 acres. In contrast, the reefs in the Southern trend are laterally larger, covering up to 700 acres, but have are typically thinner, with a maximum of 350 feet in height. The differences in reef geometries suggest that there may be differences in the relative timing of reef growth between the Northern and Southern trends.

Pinnacle reefs were formed during a period of cyclic carbonate-evaporite deposition in latest Niagaran-early Salina Time (figure 5.1). High-sea-level phases are equated to carbonate depositions (Niagara, A-1 carbonate, A-2 carbonate) and low-sea-level phases are equated to evaporate deposition (A-1 evaporite, A-2 evaporite and B evaporate) (Mesolella et al., 1974). Later, the reef and surrounding carbonates were dolomitized. The surrounding A-1 and A-2 carbonates are not uniformly dolomitized: the dolomitzation is greatest near the reef and decreases away from it. The water supplying magnesium for dolomitization was apparently forced to flow laterally to the reefs and then vertically within them. During the evaporatic cycles, the outflow water affected the evaporate deposition in the reef area, preventing the heavy brines from entering the porous reef and destroying the porosity (Jodry R., 1969).

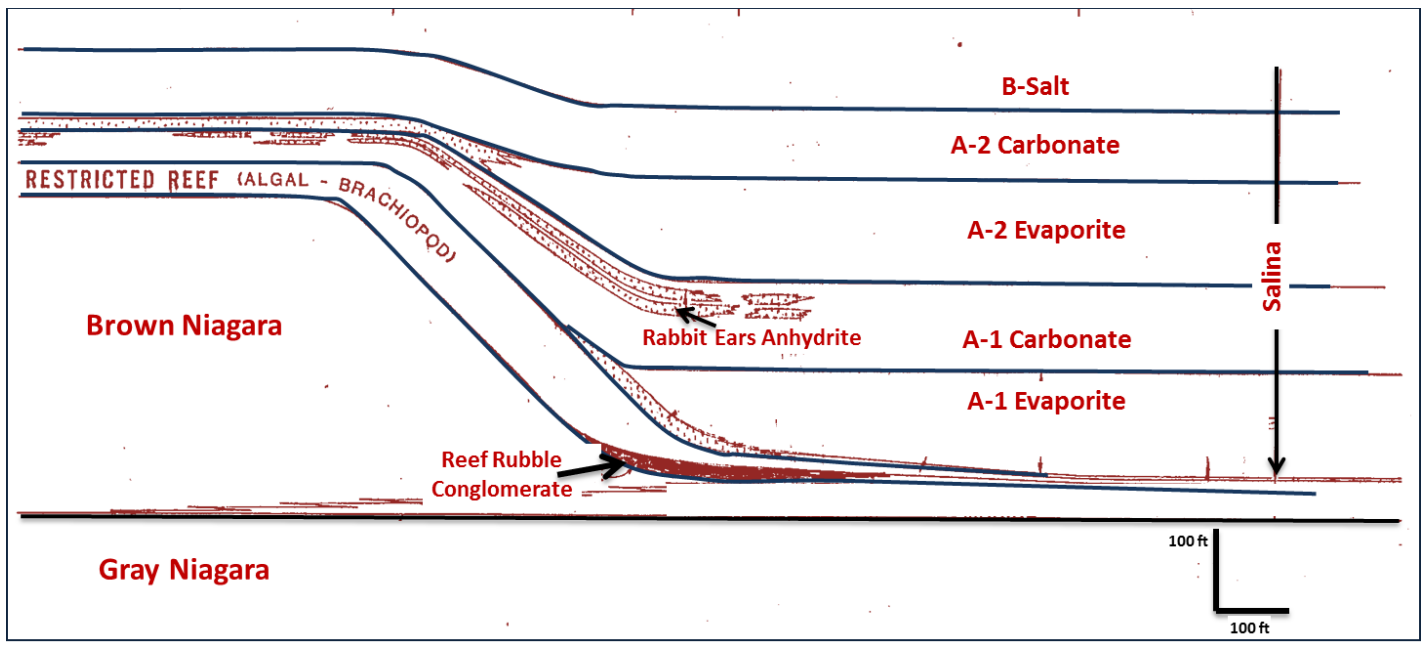

Figure 5.1: Northern Michigan Reef to Interreef Stratigraphic relationships (modified from McCollough, 1976). B-Salt, A-2 Carbonate, A-2 Evaporite and A-1 Carbonate overly the Brown Niagara (reef). Gray Niagara Top defines the base of the reef. 
Gill (1977) introduced a reef model that is divided into three stages or rock types: the top is supratidal (stromatolite), the middle is organic reef (boundstone), and the bottom is biohermal stage (wackestone). The organic reef stage is further divided into three zones: reef core, reef detritus, and vadose. The biohermal stage is in turn divided into three zones: bioherm core, crinoid-bryozoa bioherm, and bioclastic detritus. The voids, vuges, and fractures in Michigan reefs are plugged with salt and anhydrites. The anhydrite can be as a massive very finely crystalline mass with a bluish color or it can appear as large bladed crystals. The distribution of anhydrite inside the reef is primarily patchy but occasionally it may follow fractured layers, being more concentrated where there was greater fractured space available for the cement to fill (Dr. William Harrison, Western Michigan University, personal communication).

\subsection{Ray-tracing and angle coverage}

The Springdale data was recorded from dedicated monitor wells located outside the reef, at $2000 \mathrm{ft}(600 \mathrm{~m})$ interwell distance between the source well (Burch 1-20B) to the west and the receiver well (Stech 1-20A) to the east. The crosswell survey was designed to have sources and receivers with $10 \mathrm{ft}$ intervals and to cover a range of depths from $2960 \mathrm{ft}(900 \mathrm{~m})$ to $6000 \mathrm{ft}(1830 \mathrm{~m})$. The reef top is located at a depth of $4650 \mathrm{ft}(1417 \mathrm{~m})$, but is not present at either the source nor receiver well.

Because this survey extended to a deeper depth than the target zone, it allowed two types of imaging: "from above" where both source and receiver locations are at shallower depths than the reflector; and "from beneath" where sources and receivers at depths below the reflector (figure 5.2).

There are some advantages of imaging "from beneath" over the imaging "from above". In this instance, the amplitudes of reflectors from within the reef itself are generally low, but are less so for the image obtained from beneath than from above. We attribute this to attenuation and scattering of signal as it passes through the complex upper part of the reef, where gas saturation likely varies locally, reservoir pressure is extremely low, and structural complexity of the reef is evident. The image of the reef interior obtained from beneath does not pass through this until the upper edge of the image, allowing most of the reef image to be higher quality. Thus, in complex environments within hydrocarbon reservoirs, the ability to image from beneath may often be a great advantage. Of course, this involves having deep wells, deeper than the reservoir, in which to locate the sources and receivers, which is rarely the case. 


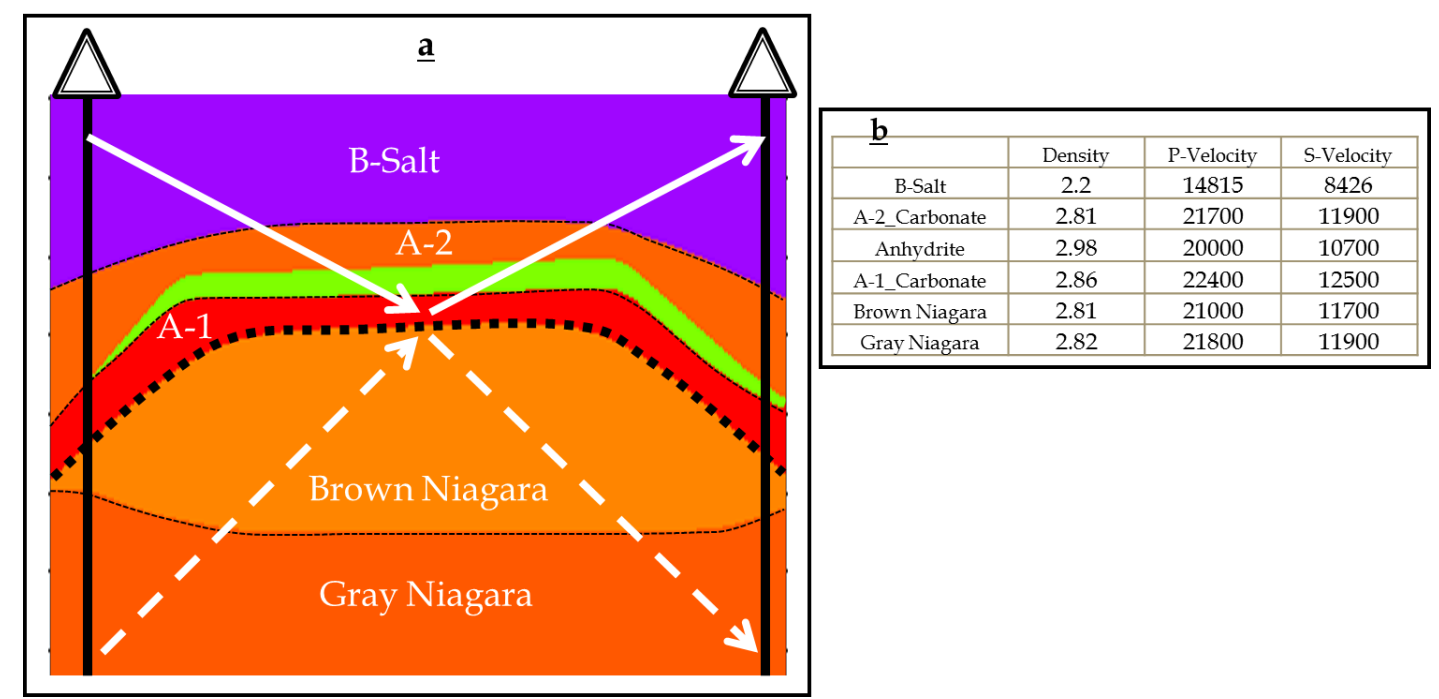

Figure 5.2: a) A schematic diagram shows two types of crosswell imaging; "from above" (solid white arrows) and "from beneath" (dashed white arrows). The background color shows the velocity model used for ray-tracing. b) The Density, Pvelocity, and $S$-velocity values for each layer used in this model and estimated from the two wells, in $\mathrm{g} / \mathrm{cc}$ and $\mathrm{ft} / \mathrm{s}$.

The reflections encountered from above are more likely to encounter critical angles than those from below because of the general trend of increasing velocity with depth. This will further tend to degrade the image from above compared with that from beneath, because the final stack may be restricted to very narrow pre-critical angle ranges in order to avoid the phase changes associated with post-critical reflections.

In addition, the dataset from beneath may provide extra information by having amplitudes from reflections over a range of angles that does not exist in the fromabove dataset (figure 5.3), especially for reflectors with shallow depths where angle ranges may be limited by acquisition constraints. 


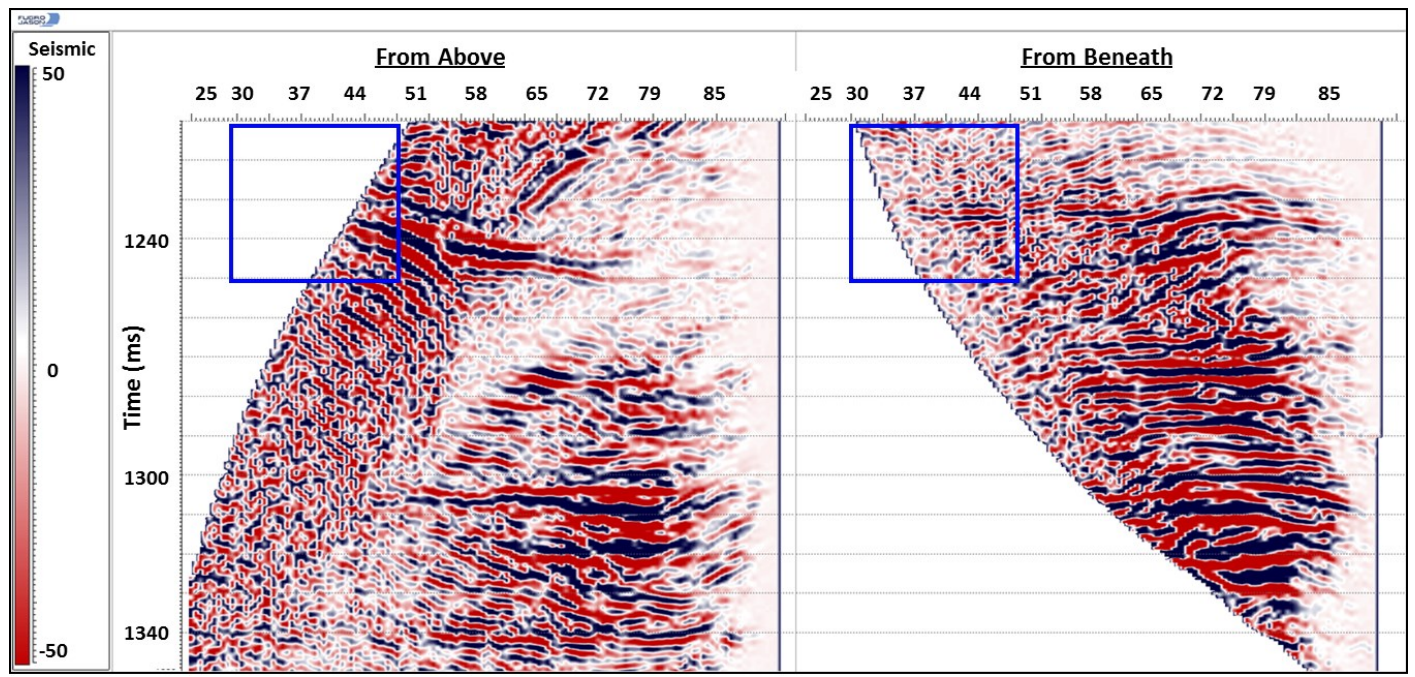

Figure 5.3: Two CDP gathers at $940 \mathrm{ft}$ (midway between the wells) for two datasets; "from above" (left) and "from beneath" (right). The blue polygon shows amplitudes recorded from beneath over angle of ranges $\left(30^{\circ}-47^{\circ}\right)$ in which recording the same from above dataset was not possible. In this example, at $1235 \mathrm{~ms}$ the from-above dataset is beyond the critical angle at $44^{\circ}$. Because the reflection from beneath does not encounter a critical angle, the image of this reflector is strong and clear.

The range of angles for any reflector is highly dependent on the velocity values in the earth (or the velocity model) as well as the geometric shape of the reflecting horizon. For instance, the minimum angle of incidence for four reflectors modeled using raytracing as shown in figure 5.4 (from top to bottom: A-2 carbonate, A-1 carbonate, Brown Niagara and Gray Niagara) shows an increase in minimum angle from A-2 to the deeper Brown Niagara reflectors while the minimum angle decreases over the Gray Niagara reflector because the velocity decreases at this interface (refer figure $5.2 \mathrm{~b}$ ). Figure 5.5 demonstrates the effect of the reef geometry on the angle coverage, especially on the reef flanks. The reef flank is seen between the source well to the left and CDP of $1600 \mathrm{ft}$ and to the right between CDP of $400 \mathrm{ft}$ and the receiver well to the right. 


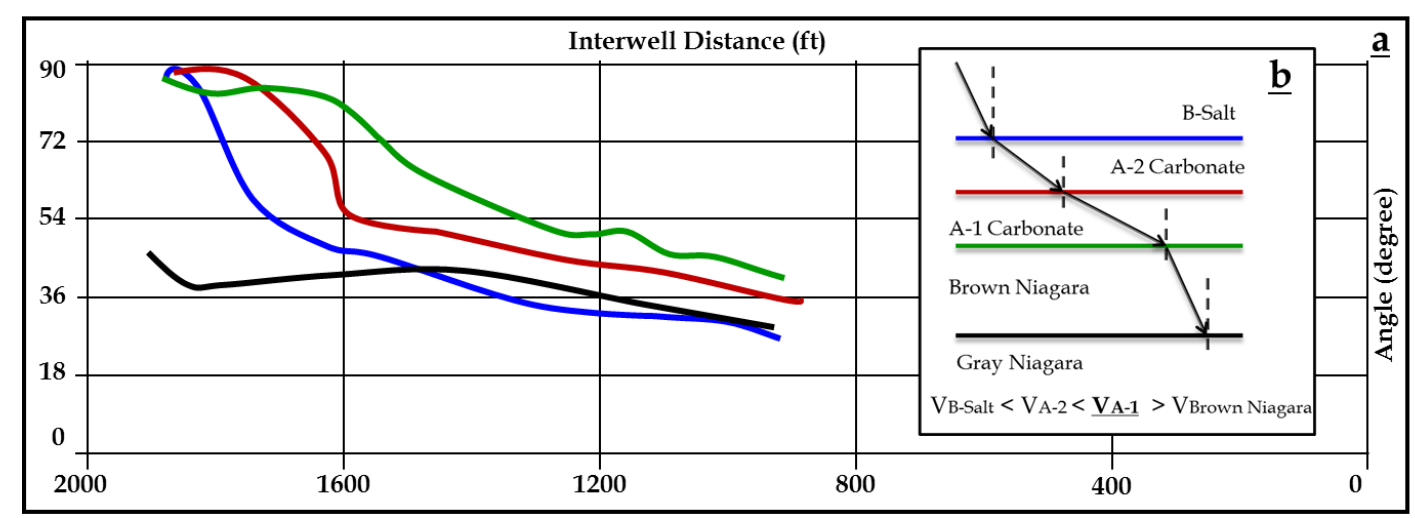

Figure 5.4: a) Minimum angles curves recorded on four reflectors, from top to bottom; A-2 Carbonate (blue), A-1 Carbonate (red), Brown Niagara (green) and Gray Niagara (black). The $x$-axis shows the interwell distance. The y-axis shows the angle recorded. The minimum angle increases from the A-2 carbonate (top) to A-1 Carbonate (lower) because the velocity increases (the ray bends away from the vertical) while the drop in the minimum angle curve from Brown Niagara to Gray Niagara is due to the drop in velocity (the ray bends toward the vertical). b) Schematic diagram for a ray path across the layers and the velocity relations of these layers. 

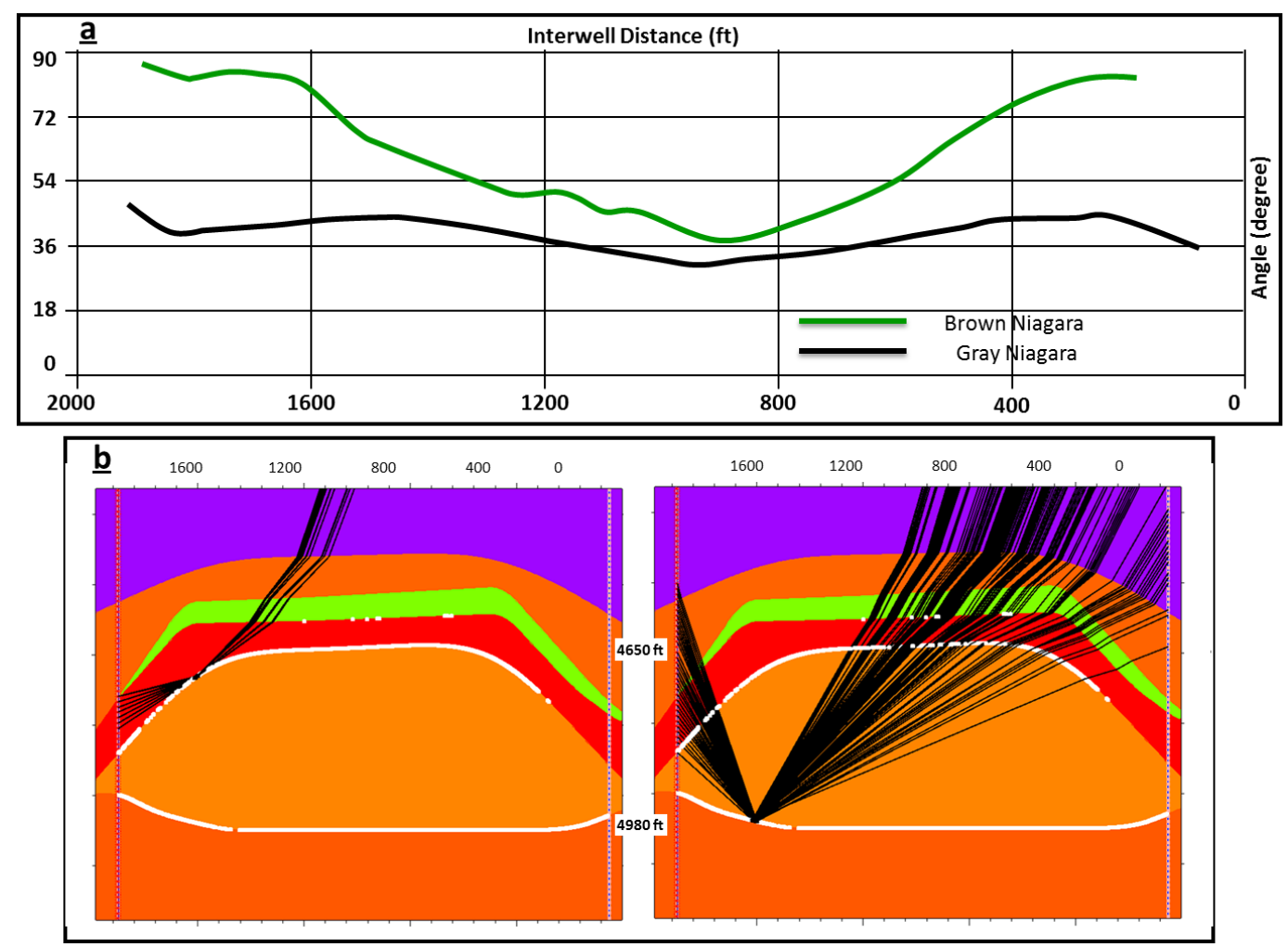

Figure 5.5: a) minimum angles for two reflectors; Brown Niagara (green line, top of reef) and Gray Niagara (black line, base of reef). b) ray paths at CDP 1640ft (reef flank) reflected from the two horizons at this location (Brown and Gray Niagara). One can notice the wide angle that the shape of the reef flanks cause to the ray paths over Brown Niagara while acute angle is occurred at the same CDP point over Gray Niagara. Also, notice the no angle coverage below $200 \mathrm{ft}$ (right hand side of the reef flank).

\subsection{AVA analysis and ideal Zoeppritz equations}

Approximations to Zoeppritz equations always assume small contrasts in elastic parameters and small angles of incidence and will fail as the critical angle is approached. Using approximations in crosswell seismic data is not sufficient because of the wide angles involved $\left(30^{\circ}\right.$ to near $\left.90^{\circ}\right)$. Despite the fact that crosswell seismic contains high-frequency data that can provide extremely high-quality images of reservoirs, often ten times the resolution exist in the surface seismic, the interpretation of crosswell data itself sometime is difficult due to some issues introduced during the crosswell imaging steps. Among these issues are: the VSP-CDP (Vertical Seismic Profile to Common Depth Point) mapping that introduces stretching-type distortions 
to the data; mapping in the presence of dips; migration artifacts introduced with the non-optimum aperture; and sensitivity to inaccurate velocities. For these reasons, comparing amplitude-versus-angle (AVA) gathers of actual crosswell data with amplitudes calculated from the solution of the complete Zoeppritz equations will provide a better understanding for the crosswell data itself and will help in most detailed AVA studies required for reservoir characterization and reservoir management.

Figure 5.6 compares the actual AVA gathers to Zoeppritz solution and helps to explain why the A-2 carbonate on the stacked sections is not as well-defined for the data from above as it is for the data from beneath. The critical angle for this reflection, from above, is $43^{\circ}$ and the data recorded is above this range. The data from below does not experience a critical and therefore avoids the associated phase change.

The gathers in figures 5.8 and 5.9 show the same behavior estimated from the exact solution of Zoeppritz equations (figure 5.7) where the amplitudes from above data start with low reflectivity at $47^{\circ}$ increasing significantly starting at about $53^{\circ}$ and continuing to $67^{\circ}$ degree before being critically refracted. From beneath, the amplitudes do not suffer from the critical angle and the amplitudes started increase at about 52 degree. No data were recorded below $52^{\circ}$ from beneath. The gather at CDP equal to $1500 \mathrm{ft}$, which is closer to the source well has a better signal to noise ratio.

In many cases, the near-critical angles may provide the only range in which a reflection can be observed due to its low amplitude at smaller angles. Of course, this will not be the case for mapping the same reflector from the other direction. In our data, because the velocity generally increases with depth, more critical angles (and narrow ranges of observable reflectivity) occur from above than from beneath. Figure 5.10 shows a case similar to that in figures 5.8 and 5.9 where the reflectivity from near-critical angle provides the only opportunity to map the reflection at the top of the A-1 carbonate. The critical angle estimated from Zoeppritz diagram $\left(63^{\circ}\right)$ matches the angle where we see distortion in phase on the actual data obtained from above. 

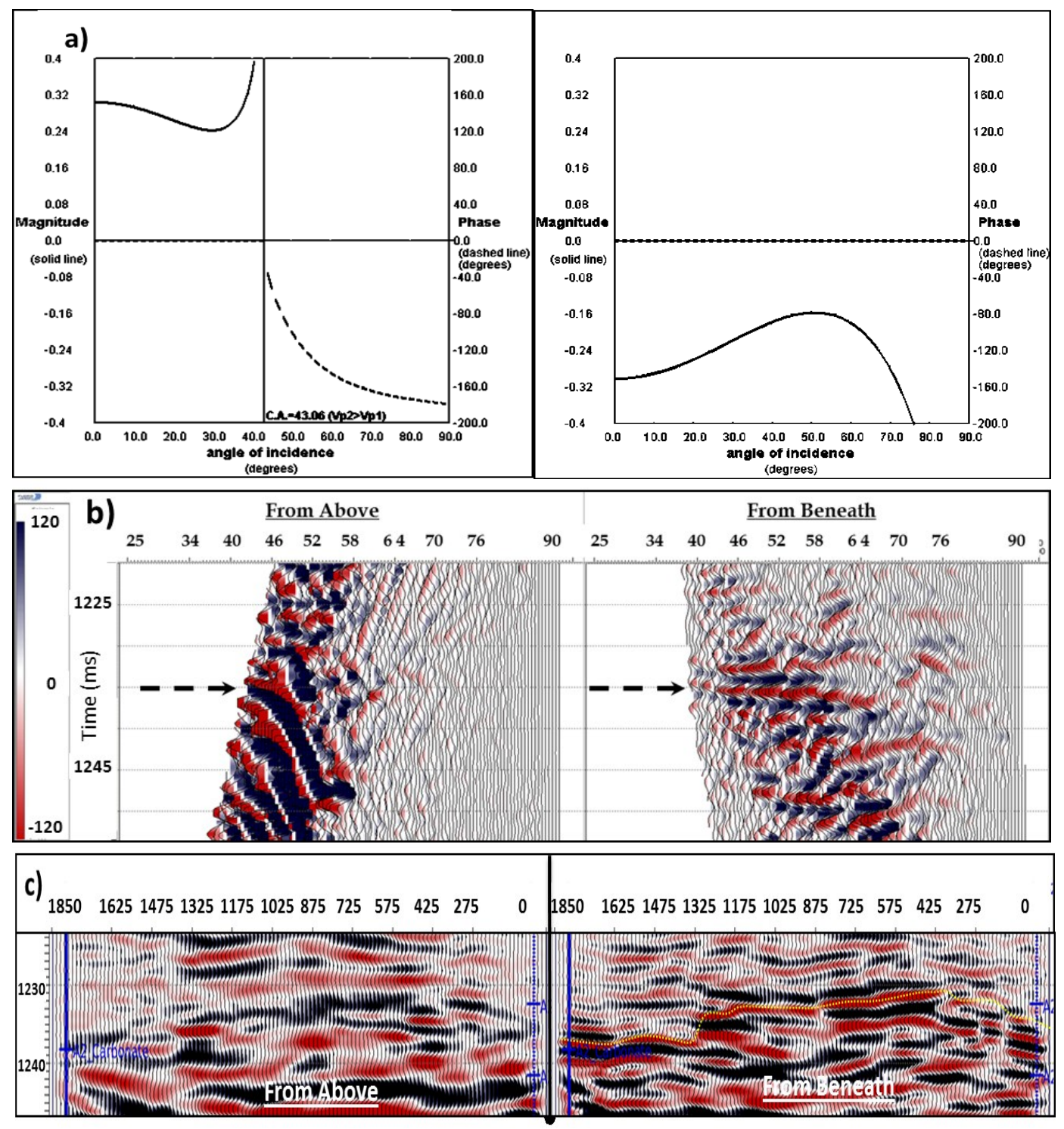

Figure 5.6: a) Zoeppritz solution for the reflector Top A-2 carbonate from above (left) and from beneath (right). Amplitudes (solid lines) and phases (dashed lines). (modified from www.crewes.org.) b) CDP gathers at $1225 \mathrm{ft}$ for above and beneath dataset. One can notice that the dataset "from above" has a critical angle at $43^{\circ}$ however by looking at the actual seismic gather there no data were recorded at that depth below $45^{\circ}$. Also, the data beyond $45^{\circ}$ suffers from phase distortion due to postcritical reflection while the data from beneath has angle range started at $38^{\circ}$ and does not experience a critical angle. The data from beneath has mis-alignment on the far angles (above 59 $9^{\circ}$ ) probably due to inaccurate velocity details used for imaging at these small wavelengths. c) Angle stacks from above and beneath for the same reflector shown in figure 5.6b. The top of A-2 carbonate is well defined from beneath compared to the same reflector from above. 


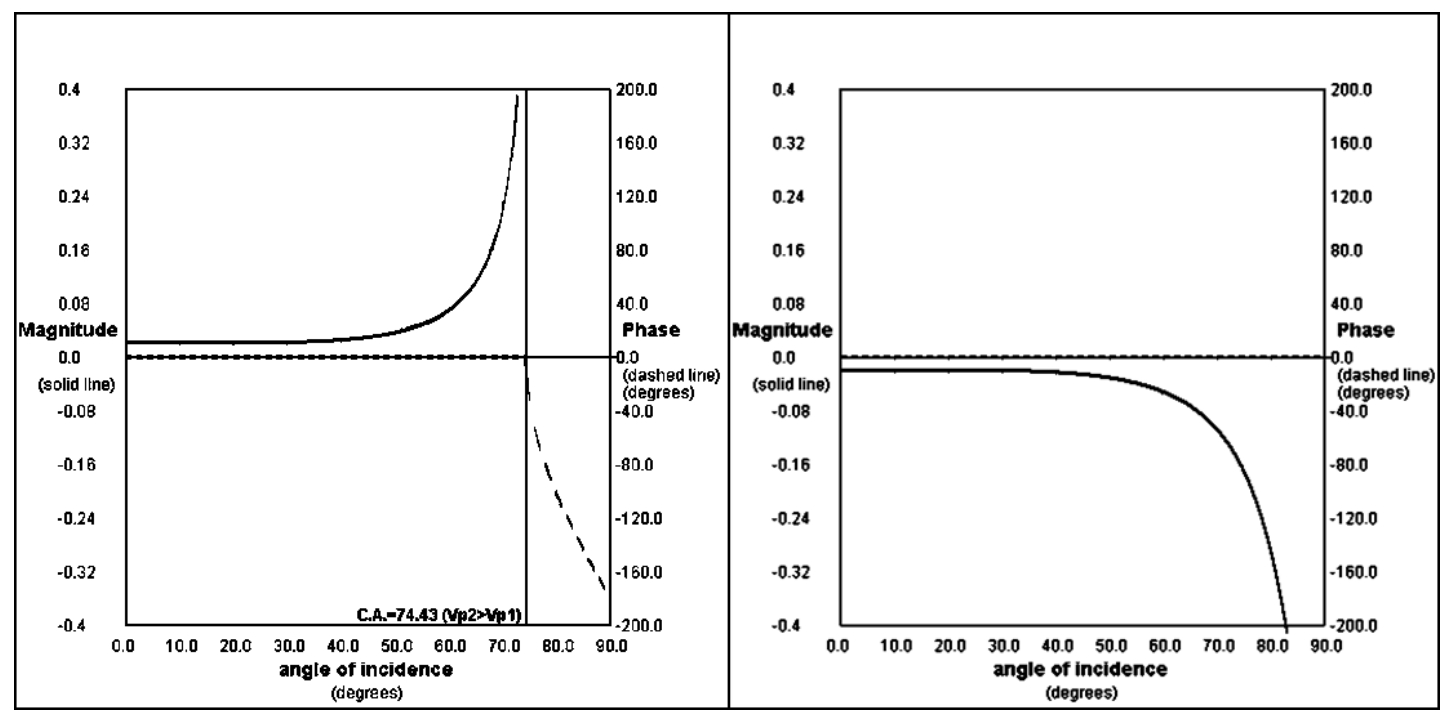

Figure 5.7: Zoeppritz solution for the base of Brown Niagara (interface with Gray Niagara); from above (left) and from beneath (right) (modified from www.crewes.org.). Amplitudes (solid lines) and phase (dashed line).

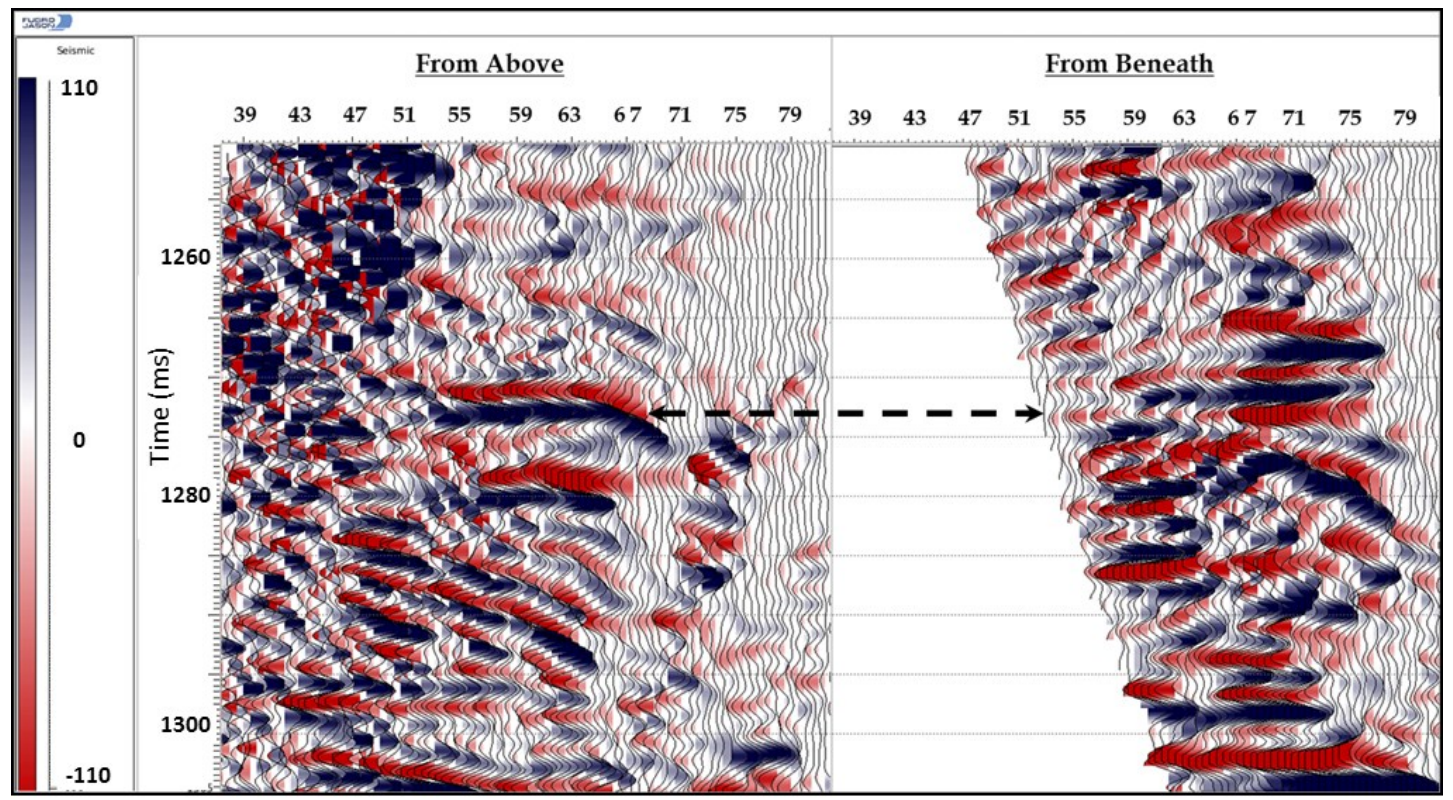

Figure 5.8: CDP gathers for the above and beneath datasets at $400 \mathrm{ft}$ (close to the receiver well). The black dashed arrow shows the location of the reflector (same interface modeled in figure 5.7) on the time axis. 


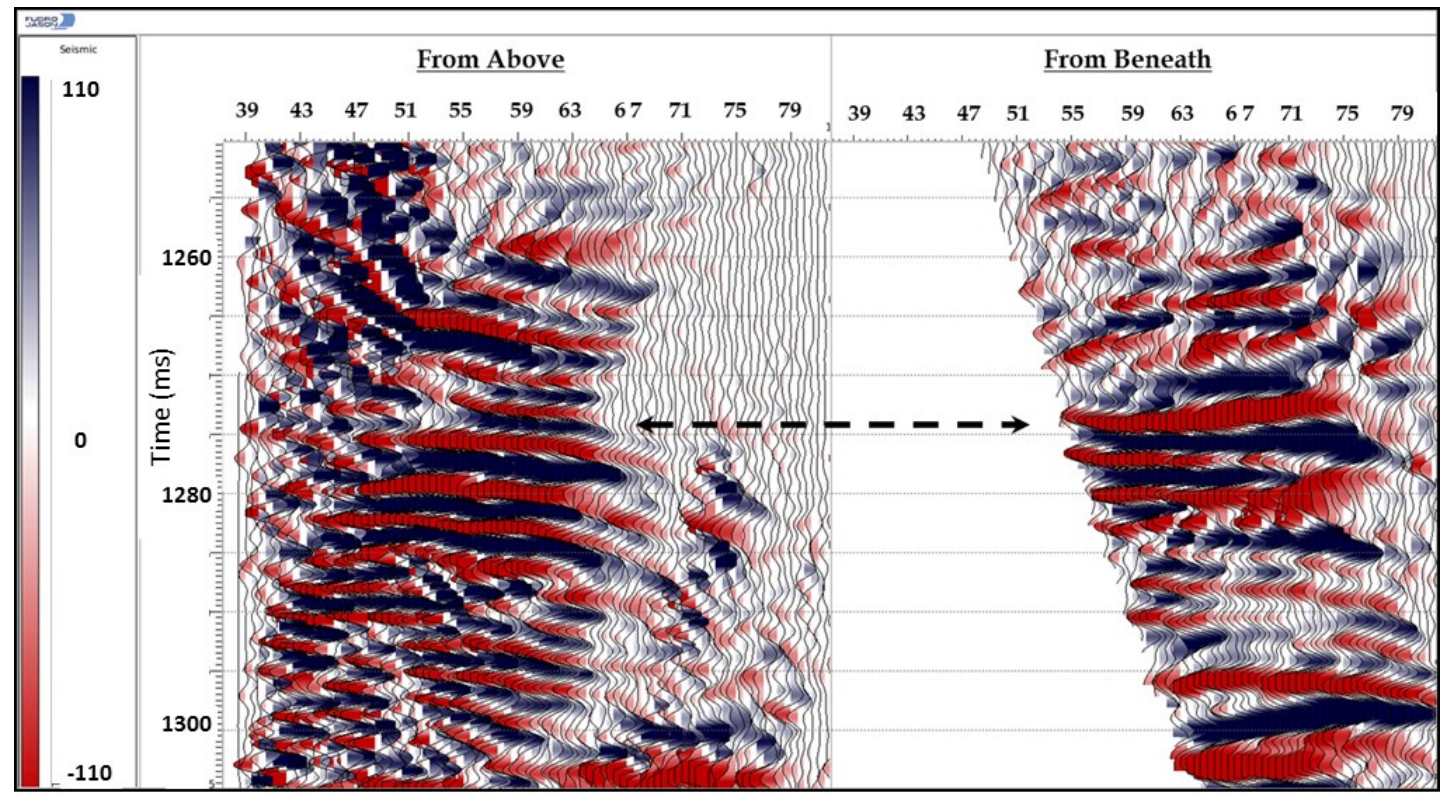

Figure 5.9: CDP gathers for the above and beneath datasets at $1500 \mathrm{ft}$ (close to the source well). The black dashed arrow shows the location of the reflector (same interface modeled in figure 5.7) on the time axis. In the Zoeppritz solution diagram (figure 5.7), the reflectivity from above is very small (0.03) at small angles, and starts to increase at about $50^{\circ}$ until reaching the critical angle at $74^{\circ}$. On the contrary, from beneath the (negative) reflectivity is also small (-0.03), but it increases starting at about $50^{\circ}$ and never experiences a critical angle. The gathers form above (figures 5.8 and 5.9), show the same behavior at small angles, with a small reflectivity at $47^{\circ}$, increasing significantly at about $53^{\circ}$ and continuing to increase to $67^{\circ}$ before apparently being critically refracted at this angle (slightly different from that predicted by our velocity model, but within reasonable errors). The gathers from beneath (figures 5.8 and 5.9), the amplitudes do not experience a critical angle and the amplitudes have already started to increase at the smallest angles recorded, about $52^{\circ}$. The gathers at $C D P=1500 \mathrm{ft}$ (closer to the source well) has a better signal to noise ratio than the gathers at $C D P=400 f$ (closer to the receiver well). 


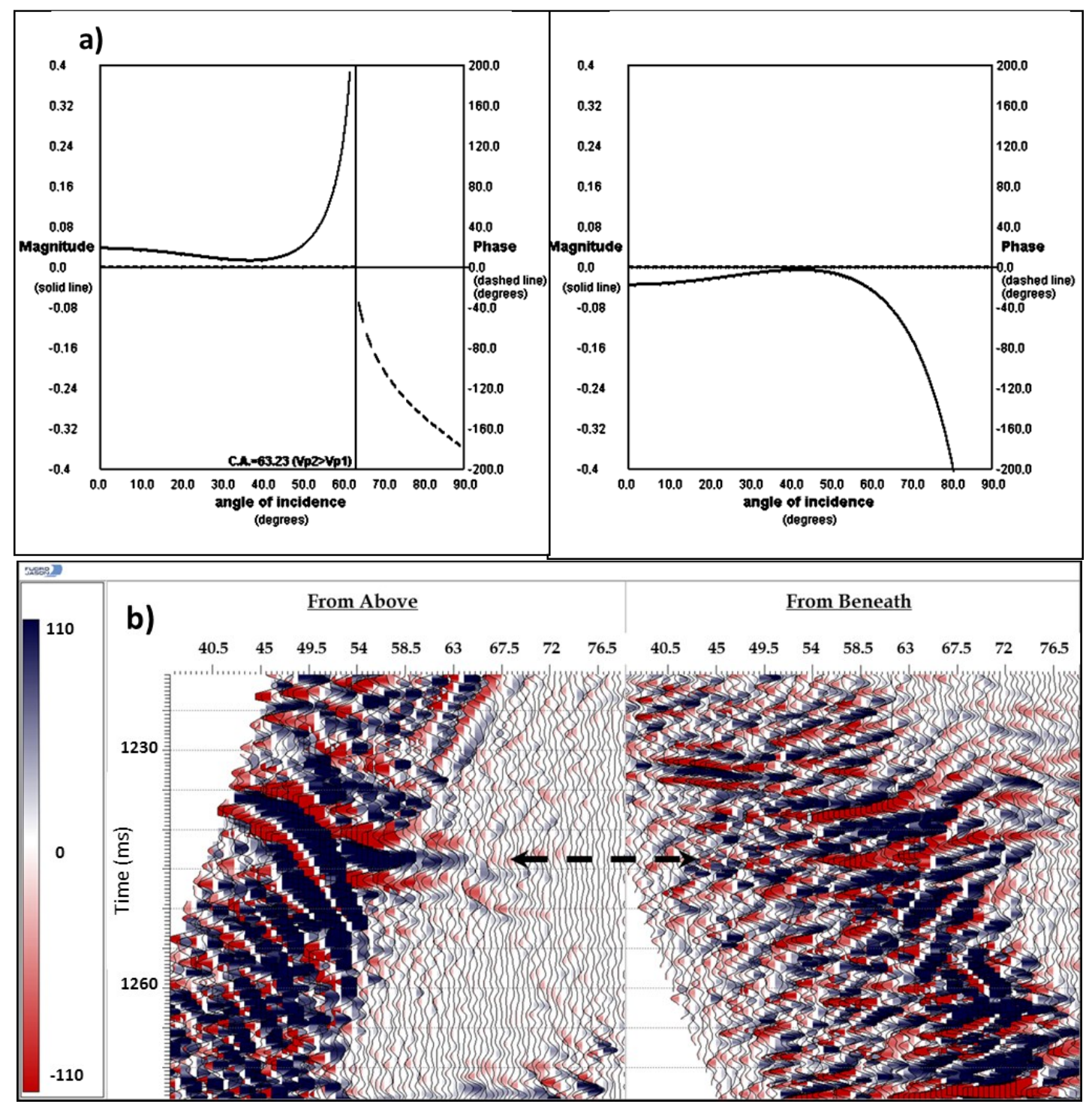

Figure 5.10: a) Zoeppritz solution for the top of A-1 carbonate: from above (left) and from beneath (right) (modified from www.crewes.org.). Amplitudes (solid lines) and phase (dashed lines). b) CDP gathers for above and beneath datasets at $1125 \mathrm{ft}$. The black dashed arrow shows the location of the reflector on the time axis. In the Zoeppritz solution diagram (figure 5.10a), the reflectivity from above is very small (0.04), approaches zero in the angle range between $30^{\circ}-45^{\circ}$ before increasing at about $50^{\circ}$ until it reaches the critical angle at $63^{\circ}$. From beneath, the (negative) reflectivity behaves similarly but with no critical angle. Due to the small reflectivity below $50^{\circ}$, it appears that the top of the A-1 carbonate is visible only within angle ranges that are near the critical angle, or at corresponding angles. From above, the reflections beyond critical angle undergo phase delays that render them essentially unusable for imaging. From beneath, the amplitudes do not suffer from the critical angle and the larger amplitudes at very large angles may be useful for imaging. 
We have noticed that, by visually looking into the reflections from above for the A-1 carbonate, there are some CDP locations between $860 \mathrm{ft}$ and $1000 \mathrm{ft}$ where the gathers indicate a change in phase due to the critical angle at wider angles $\left(\sim 75^{\circ}\right)$ than that which we estimated from Snell's law and the Zoeppritz equations $\left(\sim 63^{\circ}\right)$. There are at least two possible explanations, both involving inexact knowledge of the velocity field at sufficiently high resolution for this data set. First, the anhydrite above the A-1 carbonate is not continuous everywhere above the A-1 carbonate and the reflection we are mapping may, in these locations, be due to a direct contact of lower-velocity A-2 carbonate with the A-1 carbonate (figure 5.11). Second, the reef and surrounding carbonates are dolomitized to varying degrees, the A-1 carbonate at this location may differ from the model used. Figure 5.12 shows the Zoeppritz diagram for a reflector between anhydrite and A-1 carbonate that is less in velocity by $3 \%$ and less in density by $1 \%$, demonstrating that a very small change in properties may account for the differences observed. 

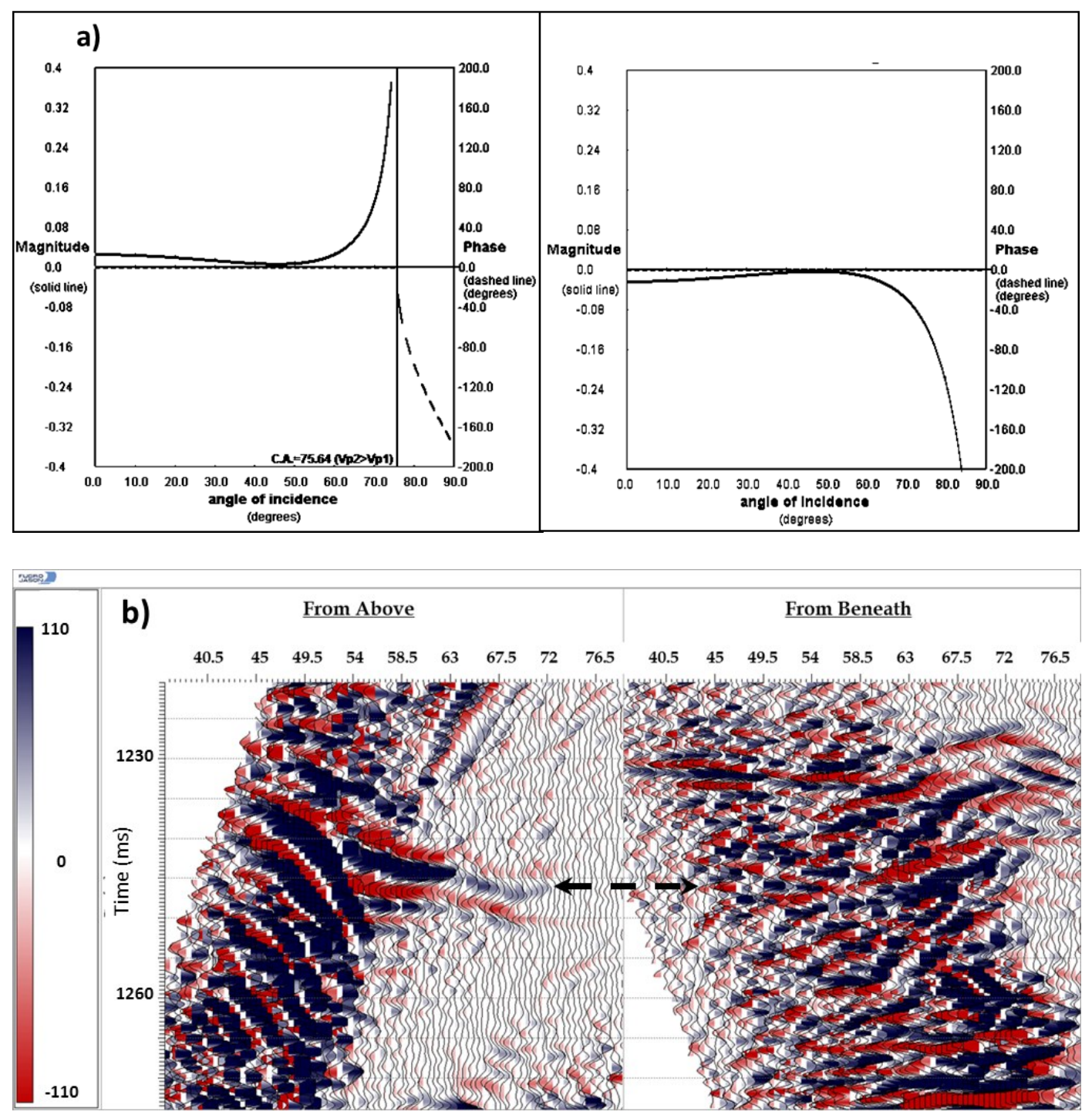

Figure 5.11: a) Zoeppritz solution for the top of A-1 carbonate, assuming that there is a direct contact between the A-1 and A-2 carbonates (anhydrite layer missing); From above is at left while the gather from beneath is at right. Amplitudes (solid lines) and phases (dashed lines). (Modified from www.crewes.org.) b) CDP gathers for above and beneath datasets at $920 \mathrm{ft}$ (close to well Shell 1-20). The black dashed arrow shows the location of the reflector on the time axis. By comparison to the previous figure (figure 5.10a), we can notice the critical angle is larger $\left(75^{\circ}\right.$, compared with $\left.63^{\circ}\right)$ and the reflectivity starts increasing later $\left(60^{\circ}\right.$, rather than $\left.50^{\circ}\right)$. 


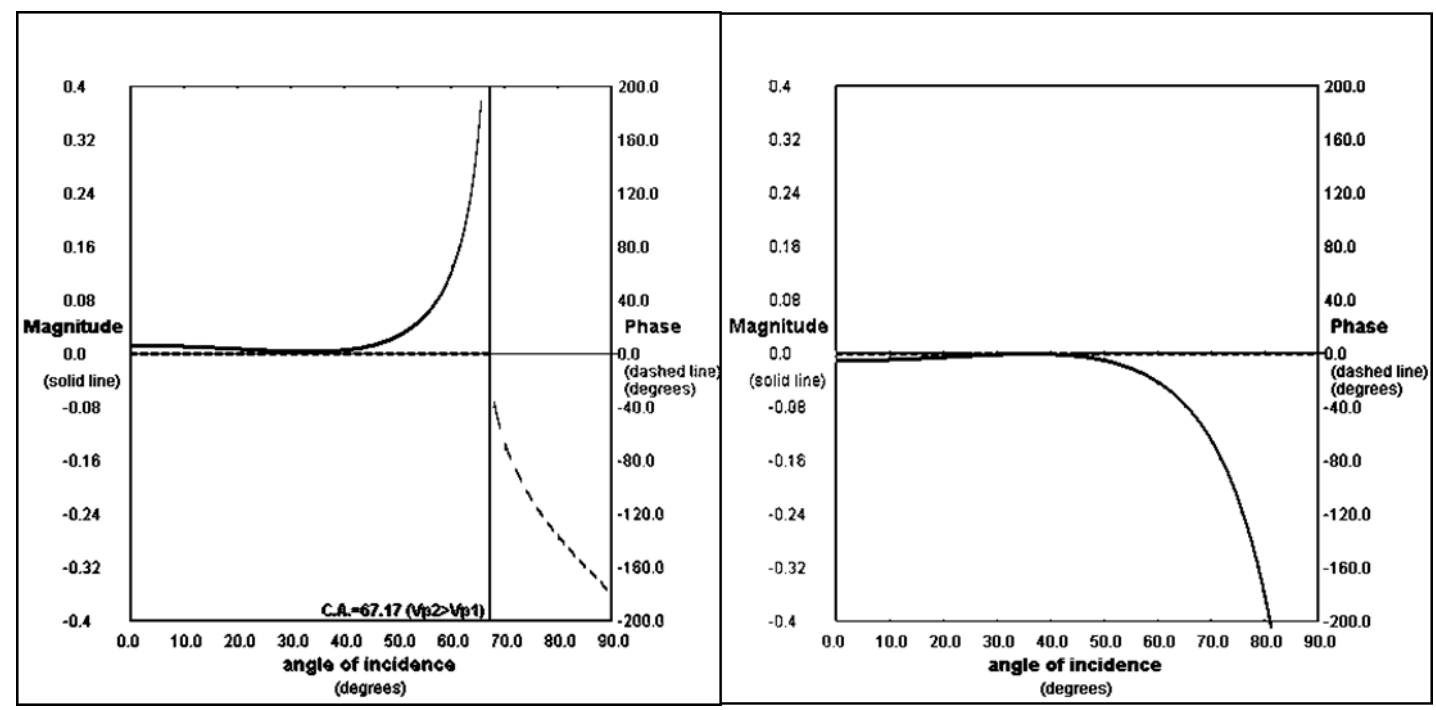

Figure 5.12: A Zoeppritz solution for a proposed reflector between Anhydrite overlying a dolomitized A-1 Carbonate (3\% slower velocity than the actual A-1); "From above" is left while "from beneath" is right (modified from www.crewes.org.). Amplitudes (solid lines) and phases (dashed lines).

\subsection{Combined model for inversion from above and from beneath}

The deterministic simultaneous AVO inversion for partial stacks (non-zero offsets) used in this work is based on the constrained sparse spike inversion (CSSI) algorithm, extended to the AVO domain. The constrained sparse spike inversion approach (CSSI), through steps of iterations, estimates the optimal elastic parameters by minimizing an objective function containing multiple terms of misfit functions. These user-control misfit functions are designed to provide a solution that honors the seismic data, keeps the sparsity criterion and constrains the results to known geology (Debeye and van Riel, 1990; Pendrel and Van Riel, 1997; Sam and Saussus, 2008).

The CSSI inversion creates a set of elastic property volumes from multiple seismic partial (angle or offset) stacks. At each CMP the seismic is modeled as the convolution of a set of reflection coefficients with the wavelet. The reflection coefficients are derived from elastic parameters using a full solution for KnottZoeppritz equation. 
The use of full Zoeppritz equations in inversion makes it more suitable for the crosswell data because the wide angles involved, and should, therefore, the inversion be valid, assuming that our input data contained sufficient breadth of angles to constrain the results. Ibrahim et al. (2010) showed that running the elastic inversion using a series of partial stacks restricted to angles less than the critical angle will avoid the complexity associated with phase rotations beyond critical angles.

The reflection AVA properties from above and from below for a specific interface must be the result of reflections from the same layer properties, albeit in different directions, and so we must be able to use this constraint to our advantage. Figure 5.11 shows the Zoeppritz diagram from above and from beneath for a generic case where the velocity increase with depth. Figures $5.11 \mathrm{a}$ and $5.11 \mathrm{~b}$ show the Zoeppritz solutions for the data that map this reflector from above and from beneath, respectively. Figure 5.11c shows the sign-reversed from beneath, where the beneath amplitudes have been multiplied by -1 (referred to hereafter as sign-reversed). Figure $5.11 \mathrm{~d}$ is showing combined curves shown in figures (a and c). For a small contrast in elastic parameters (which is the case in our study except for the B-Salt; refer to figure $5.2 \mathrm{~b}$ ), the data from above and beneath (sign-reversed) are similar within the angle ranges up to about $50^{\circ}$. Beyond this angle for the data from above, the amplitudes increase beginning several degrees before the critical angle, and experience severe phase shifts post critical angle. The data from beneath does not experience a critical angle and its amplitude continues to increase with increasing angle of incidence (figure 5.11).

If we separate the data from above into 5 partial stacks (figure 5.12), we notice that the wider-angle stacks (beyond $54^{\circ}$ ) lose details in the reef, and the overall images suffer, probably due to the difficulty of stacking phase-rotated post-critical reflections. On the other hand, the data from beneath (figure 5.13), the reef does not suffer this degradation at wide angles, but due to constraints of the acquisition geometry, the data from beneath does not have good coverage at the smaller angles. As a result, it is difficult to reliably invert either data set (from above or beneath) for full elastic properties, using a range of angles. But, together, these data sets do contain enough information for elastic inversion, when combined. 


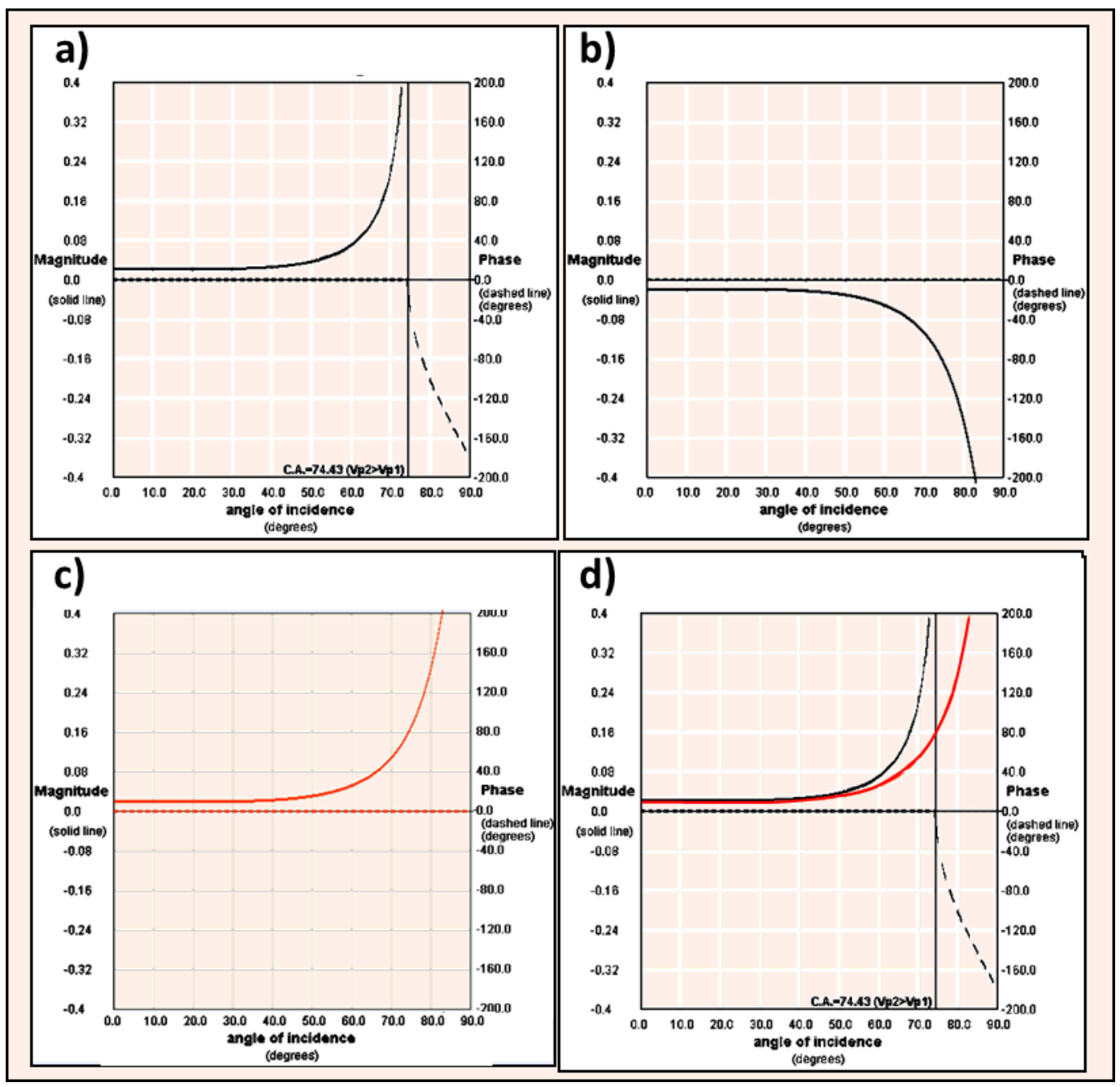

Figure 5.11: Zoeppritz diagram for a generic case in which velocity increases with depth; a) from above, b) from beneath and, c) sign-reversed from beneath. (modified from www.crewes.org.) d) A combined curves from both cases shown at a) and c). For this small contrast in elastic parameters, the data from both (from above and sign-reversed from beneath) are similar within the angle range up to $50^{\circ}$ (d). From above, the amplitudes increase to the critical angle; although not indicated on this diagram, the post-critical reflections would experience severe phase rotations. The data from beneath does not experience a critical angle and its amplitude steadily increases with increasing angle beyond about $50^{\circ}$. 


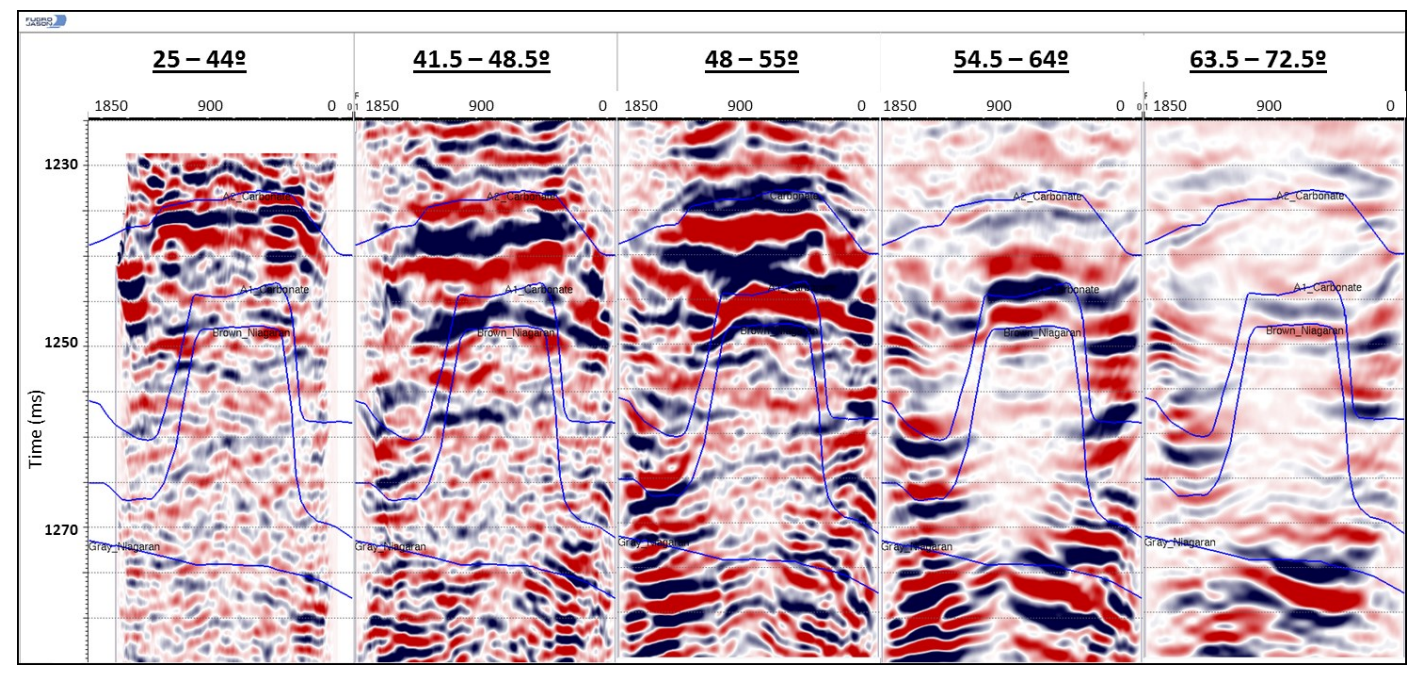

Figure 5.12: Five angle stacks for the dataset from above. At wider angles the data exhibits the increase in amplitude approaching critical angle, and phase rotation at post-critical angles resulting in poor imaging. Horizons in blue had been picked based on previous run for inversion.

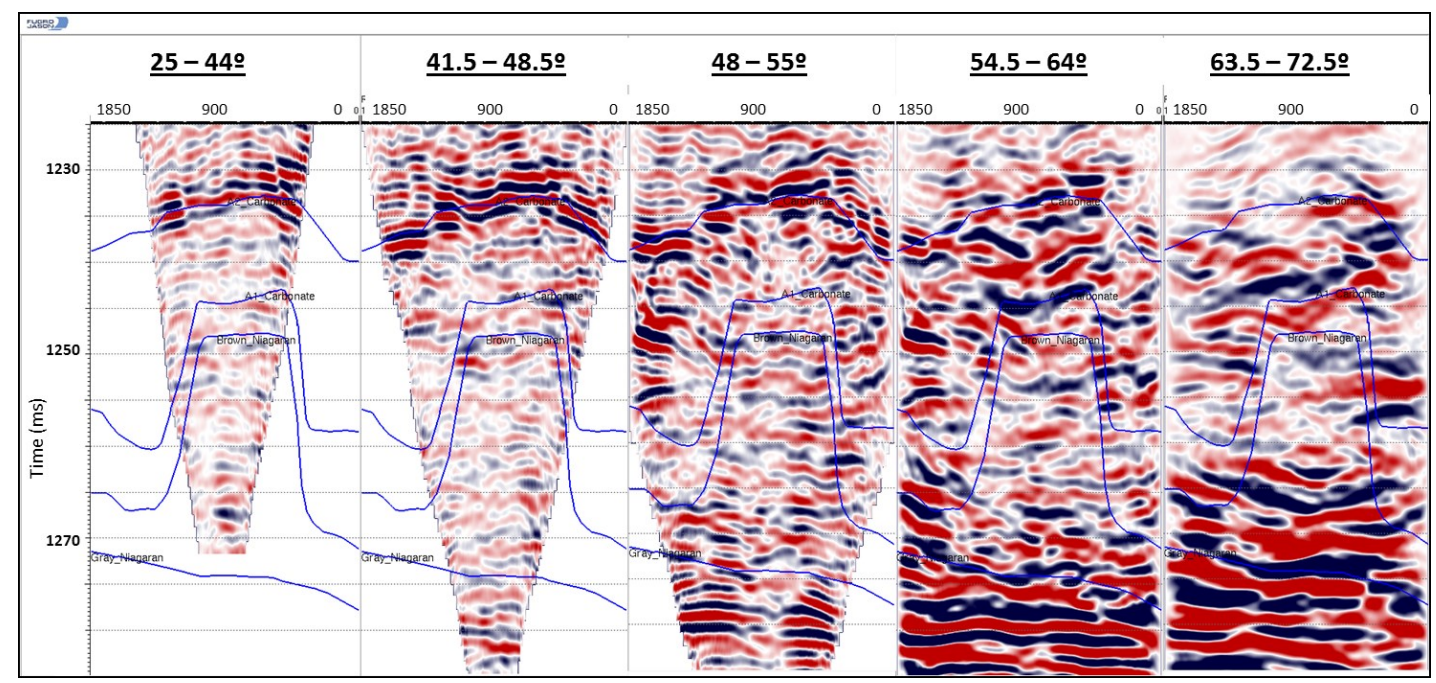

Figure 5.13: Five angle stacks for the dataset from beneath. The target reservoir (reef) shows poor coverage at small angles. Horizons in blue color picked based on previous run for inversion. 
One way to combine the data at different angles from the above and below data sets is suggested by figure 5.11: we can use the smaller angle ranges from the above dataset and add to it the data from beneath at the wider angles, treating the collected partial stacks as if they were all one set of data. Doing so, we take the advantage of the better signal in both ranges, and extending the overall angle range available for inversion. Another advantage of this approach is that the two datasets are combined in one inversion model.

However, when we discuss the "from above" and "from below" processing and images, we should notice a major issue in which the packaged inversion procedures assume that the reflection series is encountered from the top down, and therefore avoid a pitfall in assuming that straightforward use of packaged inversion suites can be used on the traces in either case without modification.

It is important to recall that, for angle range up to about $50^{\circ}$, the reflection observed from above is the same amplitude of the reflection observed from below, but with opposite sign. Yet, for all angles beyond about $50^{\circ}$, the amplitude will vary strongly, depending on which layer is encountered before the interface, and which after (figure 5.11d). So a simple sign change, at any angles beyond $50^{\circ}$, is not sufficient.

Our approach to use the small-angle gathers from above with the large-angle gathers from beneath is by "flipping" the seismic traces of both data sets; from above and from beneath (top-down, or last-sample to first-sample) so that the inversion can take place from beneath. The flipping process results in traces referred to hereafter as "time-reversed" traces. Then, following the time-reversal of the seismograms, we sign-reversed the data (gathers) from above, so that they are (nearly) equivalent to the appropriate gathers would have been from beneath. Then we performed the inversions using standard software, and finally time-reversed the resulting image back (flip) so that the shallow depths are again on top. This approach will honor the sign of the small-angle gathers after switching the time order (as if they were being reflected from beneath, which figure $5.11 \mathrm{~d}$ shows that it is valid for small angles - below $50^{\circ}$ ), but it will also honor the Zoeprittz solutions for the large-angle gathers, since it will be correct for from-beneath inversion.

Figure 5.14 shows six angle stacks: three from above (pre-critical angle range) and three from beneath (wider angles) with overlapping angle ranges. Figure 5.15 shows the same as figure 5.14 but with time-reverse (top-down, or last-sample to firstsample) all angle stacks (stacks 1 to 6). The pre-critical angle stacks (from left; stacks 1 to 3 ) also underwent sign-reversal by multiplying their amplitudes by (-1). These 6 stacks (figure 5.15) are used together in simultaneous inversion, as if the data were all collected from beneath. The P-Impedance and S-Impedance results before time-reversing them back are shown in figure 5.16, while the same images after timereversing the inversion results are shown in figure 5.17 . 


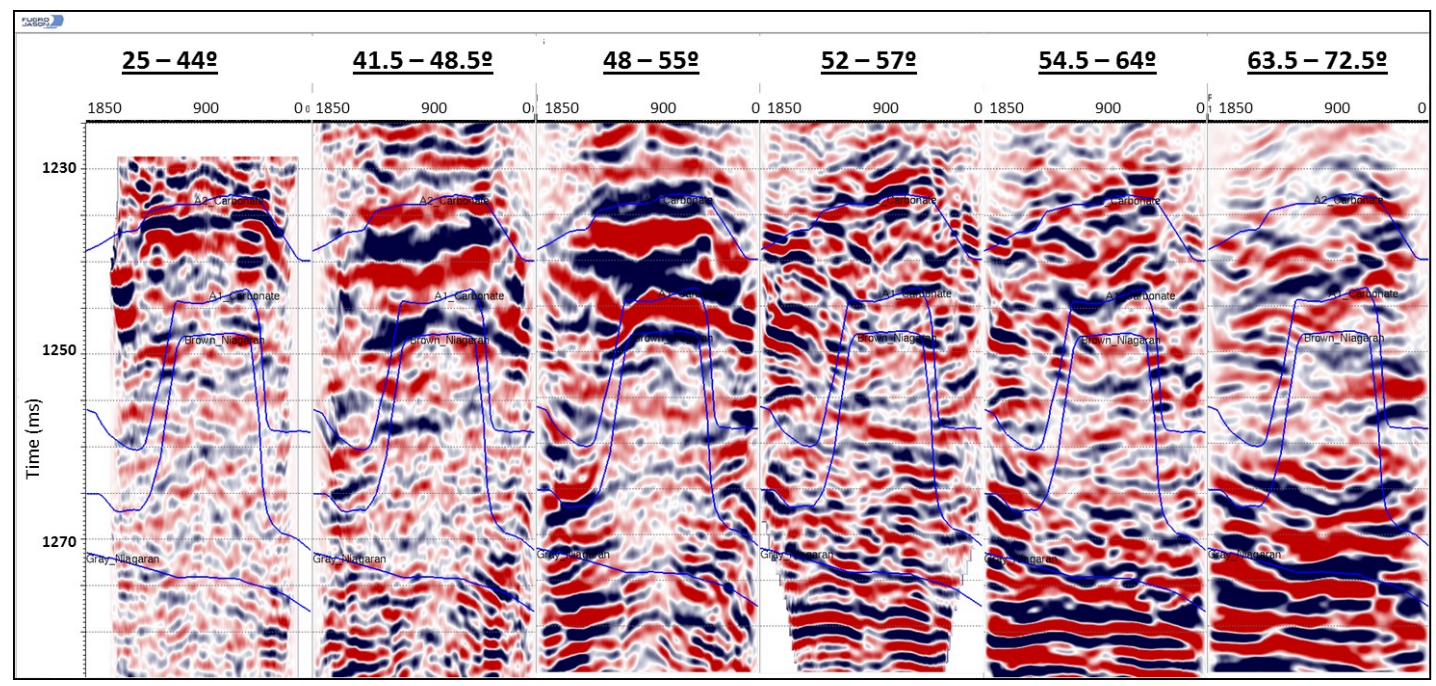

Figure 5.14: 6 angle stacks combined from above (from left; stacks 1 to 3) and from beneath (from left; stacks 4-6) with overlap angles. Horizons in blue color picked based on previous run for inversion.

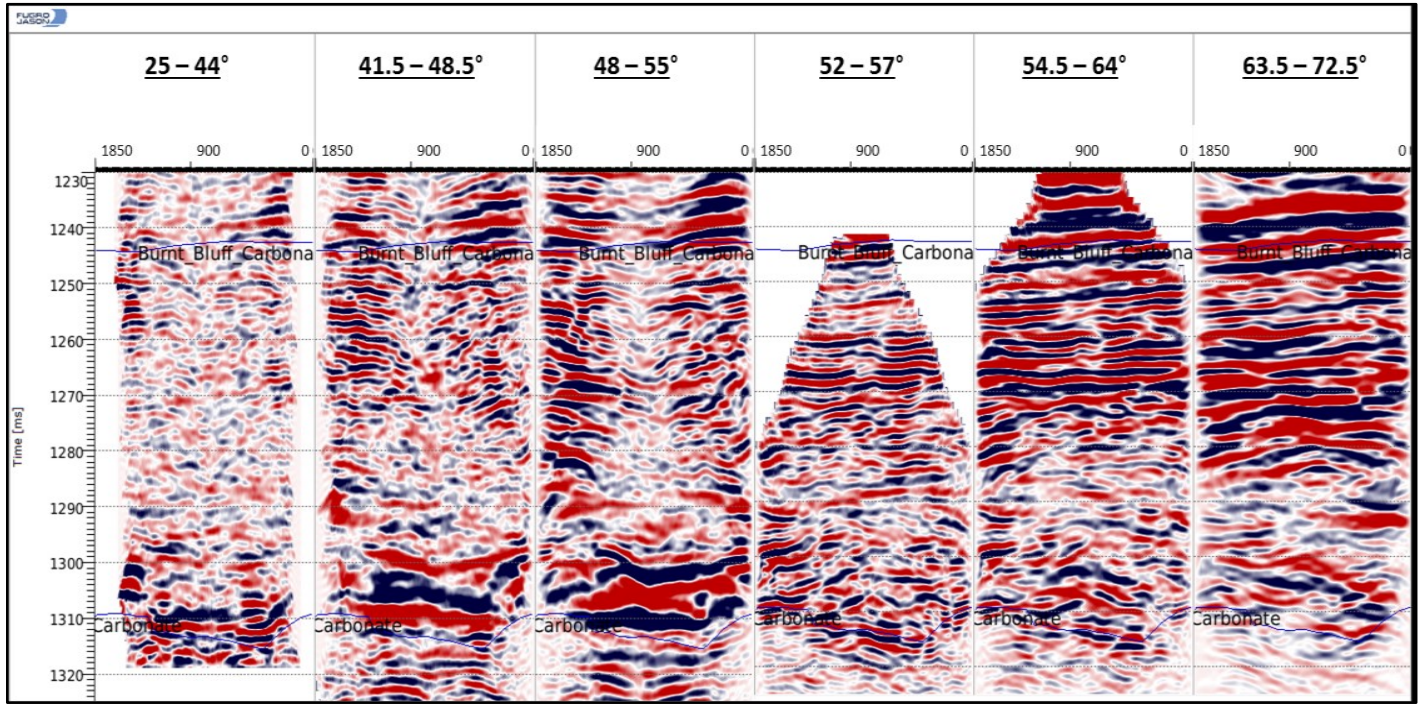

Figure 5.15: Same as previous figure but with Time-reversed samples for all the stacks from above and from beneath and sign-reversed samples only for the stacks from above (from left; stacks 1 to 3). Horizons in blue color picked based on previous run for inversion. 


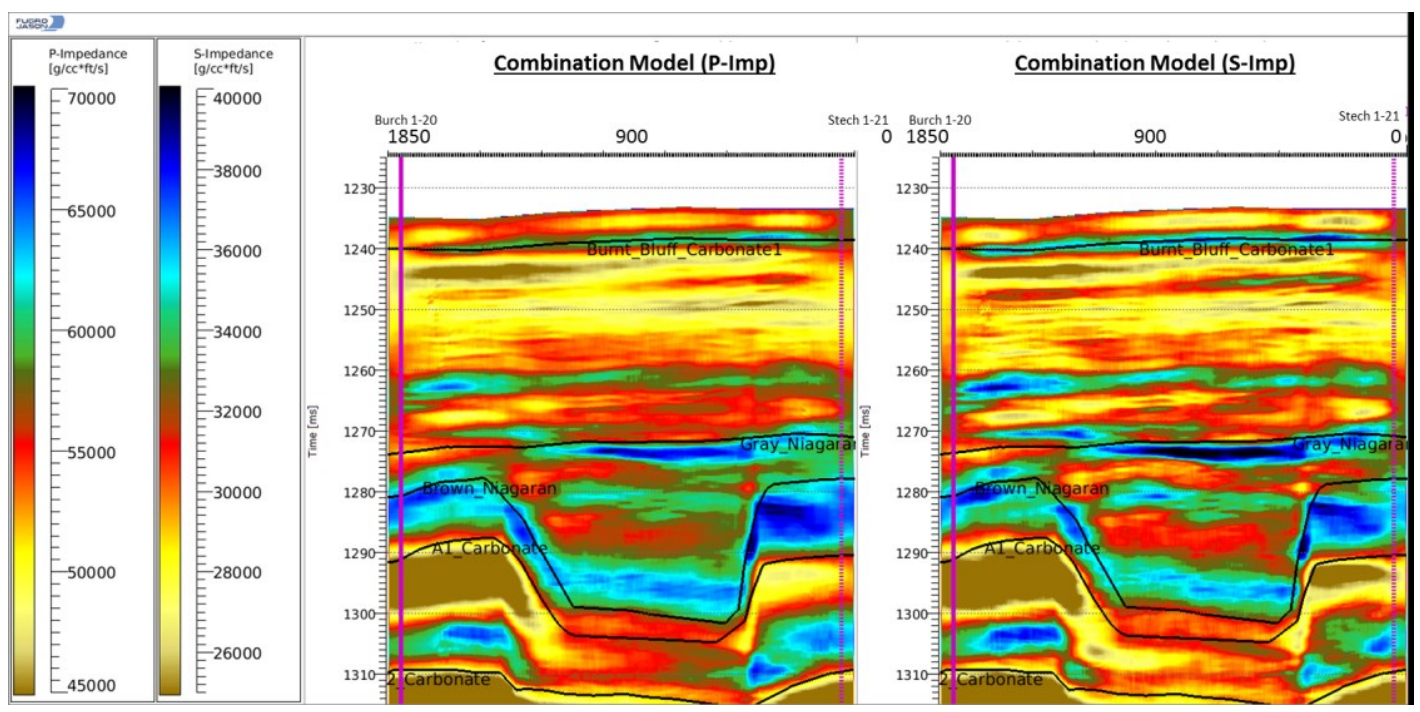

Figure 5.16: Time-revered inversion results using combined approach for PImpedance (left) and S-Impedance (right)

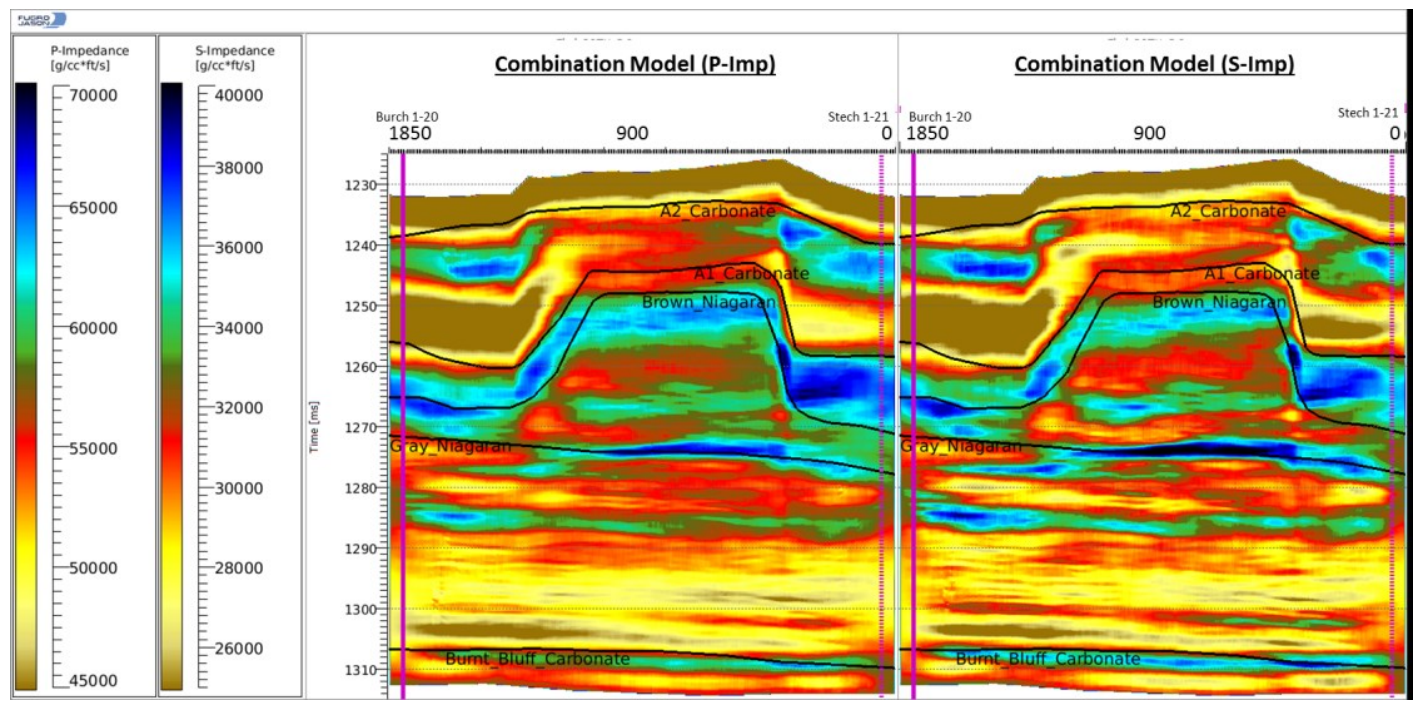

Figure 5.17: Inversion results using combined approach for P-Impedance (left) and S-Impedance (right). Notice the images flipped back as a final step.

Since the S-Impedance is more sensitive than the P-Impedance to the wider angles used in this crosswell experiment, a comparison between the combined approach SImpedance with the results from individual runs from above and from beneath is shown in figures 5.18 (for the inversion of data from above) and 5.19 (beneath). 
For figure 5.18, the combined inversion result exhibits more detail than that from above, and appears to show the distribution of internal layers that is reasonable for this reef, matching the accepted geological reef model better. The results from above offer lower apparent resolution, and may be driven by the strong amplitudes near the critical angles and/or post-critical reflections that had not been avoided by the limited angle ranges chosen. For figure 5.19, the combined results look smoother and less noisy than from beneath. We believe the increased noise in the inversion from beneath results come from a lack of data in the lower angle ranges (below $48^{0}$ ).

In general, it seems that the data from above is more driven by the frequency with which the critical angle is encountered while the data from beneath is not subject to that, but suffers, to a lesser extent, from limited small-angle coverage.

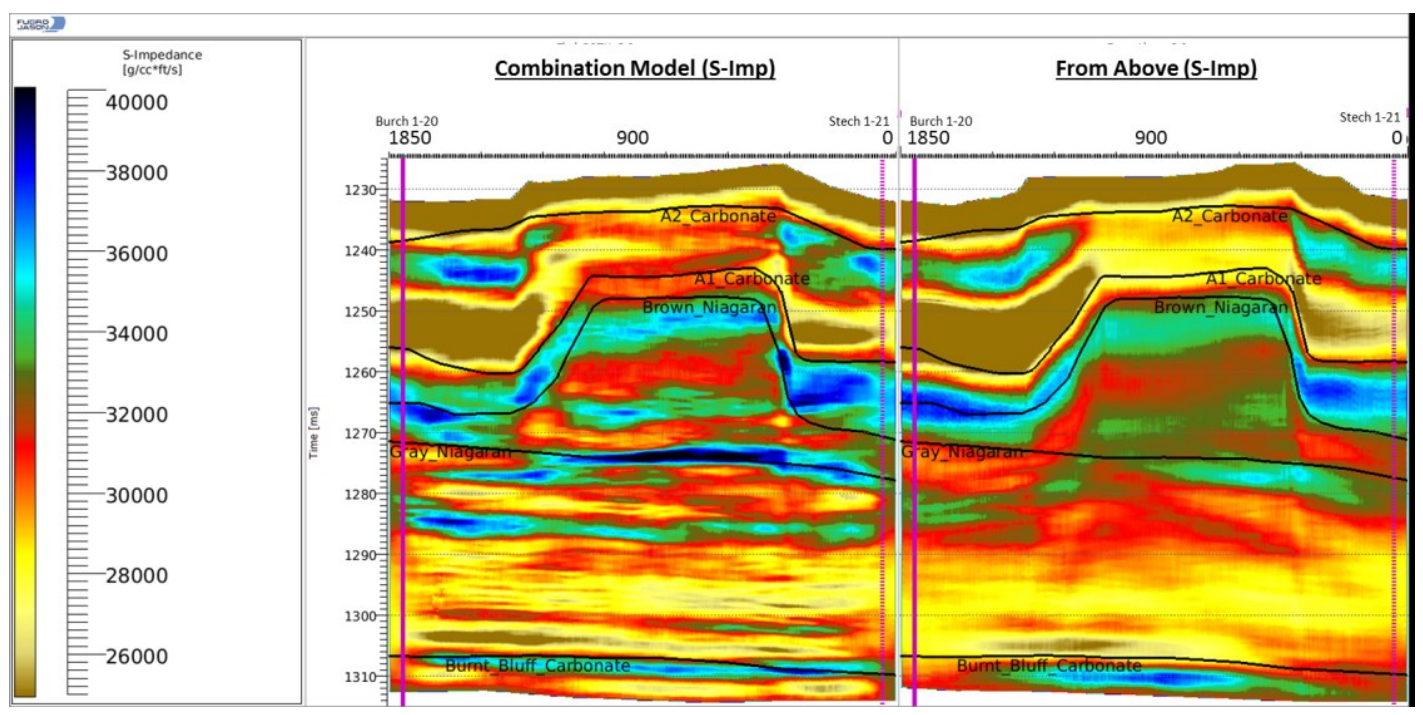

Figure 5.18: S-Impedance results using combined approach (left) versus from above only (right). The combined exhibits more detail and seems to show a distribution of internal layers that is reasonable for this reef and matches the known geological reef model. The results from above are likely driven by the strong amplitude near-critical angle reflections. 


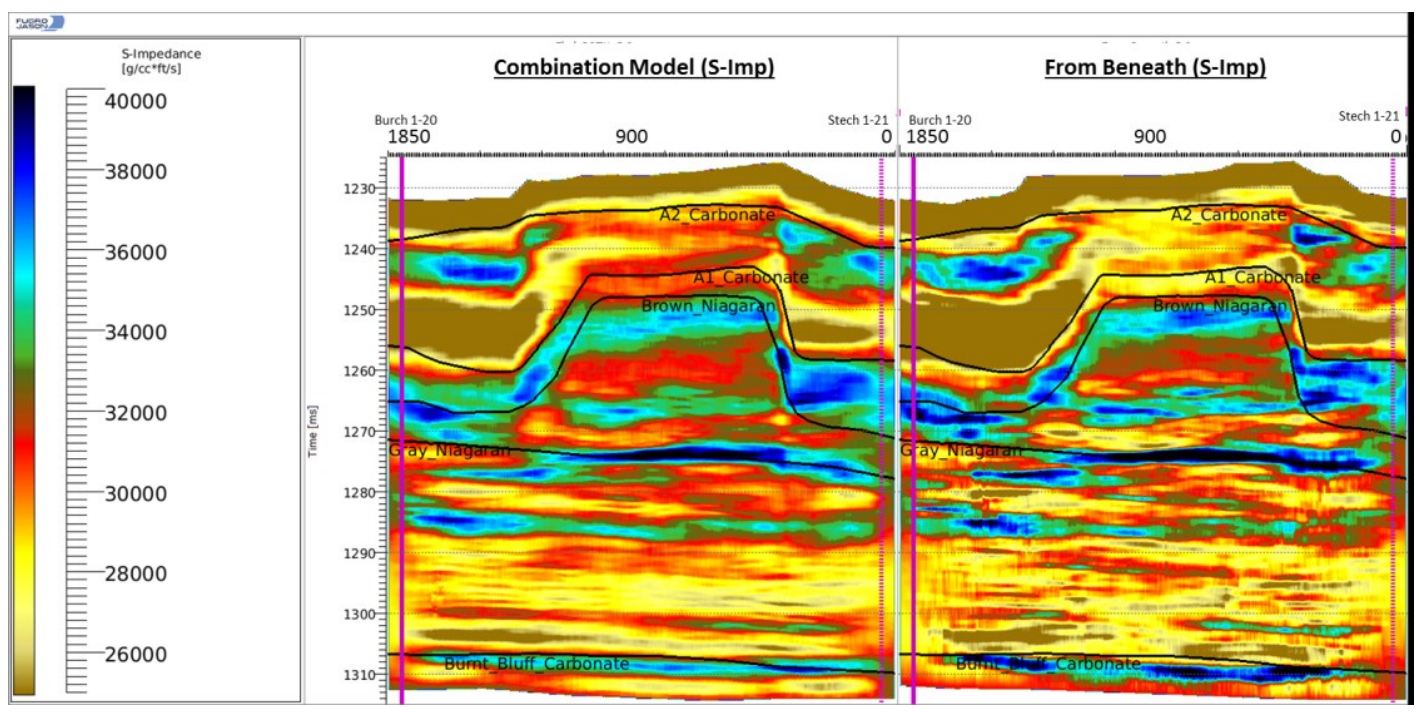

Figure 5.19: S-Impedance results using combined approach (left) versus from beneath only (right). The combined inversion looks smoother and less noisy. We believe the noise in the beneath inversion results from limited coverage at small angle ranges (below $48^{\circ}$ ).

\subsection{Conclusion}

Crosswell seismic imaging in combination with deterministic inversion has demonstrated its usefulness in reservoir characterization by providing a high quality image with detailed internal features within the reef that can be used for further reservoir management decisions.

The image from beneath does not pass through the complex structure at the top of the reef, allowing most of the reef image to be higher quality than the image from above. Thus, in complex environments within hydrocarbon reservoirs or in areas of high attenuation, the ability to image from beneath may often be a great advantage; of course, this involves having wells deeper than the target in which to locate the sources and receivers.

The frequency with which the velocity increases with depth across any reflecting interface results in the fact that the dataset from beneath will experience fewer postcritical reflections, with their phase rotations, compared to the dataset from above, and should provide useful data for analysis and imaging at wider angles. 
The interpretation of crosswell images is difficult, in some cases, due to the stretching distortion introduced by the VSP-CDP mapping algorithm, so comparing the amplitude-versus-angle (AVA) gathers of actual crosswell data with exact solution of Zoeppritz equations provides a better understanding for the crosswell data itself and will help in most detailed AVA studies.

A comparison of the AVA gathers to the Zoeppritz solution for the reflection at the top of the A-2 carbonate, which is better-defined for the data from beneath than from above suggests the possibility that the anhydrite above A-1 carbonate may not be continuous across the image.

For a small contrast in elastic parameters between layers (which is the case in this study except at the B-Salt), the data from above and beneath are similar within the angle range up to $50^{\circ}$. The data from above increases in amplitude near the critical angle and undergoes phase rotation at post-critical angles, while the data from beneath may not have enough coverage at small angles at the reservoir depth for elastic inversion. The reflection AVO properties from above and below for a specific interface must be the result of the same layer properties, and so we take advantage of this fact in a combined inversion approach in which smaller angles are obtained from the above dataset and wider angles (sign-reversed) from the beneath dataset.

The results of elastic properties of combined approach seem to give better results compared to the individual runs of the above and beneath data. The results from the combined inversion exhibit more apparent detail and provide a result that is reasonable for this reef and matching the Gill's geological reef model. 


\subsection{Reference}

Caers J., 2005, Petroleum Geostatistics, Society of Petroleum Engineers, 88 pages.

Carrillo P., Milagrosa A., Bryans B., and Turpening R., 2007, Attenuation coefficient tomogram and Q distribution image from crosswell survey in the Northern Reef Trend of Michigan Basin; SEG Expanded Abstracts 26, 1252. DOI:10.1190/1.2792731

Caughlin, W.G., F.J. Lucia, N.L. McIver, (1976), The Detection and Development of Silurian Reefs in Northern Michigan, Geophysics, Vol. 41, No. 4

Connolly P., 1999, Elastic impedance: The Leading Edge, VOL. 18, NO. 4, P. 438 452.

Connolly, P. and Kemper, M., 2007, Statistical uncertainty of seismic net pay estimations. The Leading Edge, October 2007, 1284-1289.

Contreras A., Verdin C. T. and Fasnacht T., 2006, AVA simultaneous inversion of partially stacked seismic amplitude data for the spatial delineation of lithology and fluid units of deepwater hydrocarbon reservoirs in the centeral Gulf of Mexico: Geophysics, VOL 71, NO. 4, P. E41-E48.

Cumings, E.R. (1932), Reefs or Bioherms? Geol. Soc. America Bull., Vol. 43, No. 1

Debeye, H.W. J., and P. van Riel, 1990, Lp-norm deconvolution: Geophysical Prospecting, 38, 381-404.

Deutsch C.V., 2002, Geostatistical Reservoir Modeling, Oxford University Press, 376 pages.

Droste, J.B., R.H. Shaver, (1985), Comparative Stratigraphic Framework for Silurian Reefs-Michigan Basin to Surrounding Platforms, in Special Paper \#4 Ordovician and Silurian Rocks of the Michigan Basin and its Margins, Mich. Basin Geo. Soc., Lansing, Mich.

Dubrule, O., 2003, Geostatistics for seismic data integration in earth model. Distinguished Instructor, Series, No. 6.

Gill, D., 1973, Stratigraphy, facies, evolution and diagenesis of productive Niagaran Guelph reefs and Cayugan sabkha deposits, the Belle River Mills gas field, Michigan basin: Ph.D. Dissertation, University of Michigan, Ann Arbor, 275 p. 
Gill, D., 1977, The Belle River Mills gas field: Productive Niagaran reefs encased by sabkha deposits, Michigan basin: Michigan Basin Geological Society Special Paper 2, $188 \mathrm{p}$.

Hill, S., 2005, Inversion-based thickness determination: The Leading Edge, 24, 477480.

Huh, J.M.(1973), Geology and Diagenesis of the Niagaran Pinnacle Reefs in the Northern Shelf of the Michigan Basin, Ph.D. thesis, Univ. of Michigan, Ann Arbor, Michigan

Ibrahim, M. S, Pennington, W. D. and Turpening, R. M, 2009, Crosswell seismic imaging and inversion of a Michigan reef from above and from beneath: SEG Expanded Abstracts 28, 3934-3938

Ibrahim, M. S, Pennington, W. D. and Turpening, R. M, 2010, Crosswell seismic imaging of acoustic and shear impedance in Michigan reef: The Leading Edge, June, vol. 29 , no. 6 , p. 706-711

Jarvis, K., Folkers, A. and Saussus, D., 2007: Reservoir compartment prediction of the Simpson field from geostatistical inversion of AVO seismic data: ASEG Extended Abstract 2007, Perth, Western Australia, p. 1-3

Jodry, R. L., 1969, Growth and dolomitization of Silurian reefs, St. Clair County, Michigan: Bulletin of the American Association of Petroleum Geologists, Vol 53, No., 4, April, 1969, PP. 957-981

Latimer, R. B., Davison, R. and P. van Riel, 2000, An interpreter's guide to understanding and working with seismic-derived acoustic impedance data: The Leading Edge, 19, 242-256.

Lazaratos. S. K., 1993, Cross-well reflection imaging: Ph.D. Thesis, Stanford University.

Lazaratos, S. K., Rector, J. W., Harris, J. M., 1991, High resolution cross-well reflection imaging: 61st Annual International Meeting, SEG, Expanded Abstracts, 350-353.

Lowenstam, H.A., (1950), Niagaran Reefs of the Great Lakes Area, Jour. of Geology, Vol. 58

Lu, S. and McMechan, G, 2004, Elastic impedance inversion of multichannel seismic data from unconsolidated sediments containing gas hydrate and free gas: Geophysics, Vol. 69, No. 1 (January-February 2004); P. 164-179, 
Mesolella, K., J., Robinson, J.D., Mccormick, L.M. and Ormiston, A.R., 1974, Cyclic deposition of Silurian carbonates and evaporates in Michigan basin: Bulletin of the American Association of Petroleum Geologists, Vol 58, No., 1, January, 1974, PP. $34-62$

Nelson, H.F., C.W. Brown, J.H. Brineman (1962), Skeletal Limestone Classification in Classification of Carbonate Rocks, Amer. Assoc. Petrol. Geol., Mem. 1

Pendrel John, 2006, Seismic inversion - A critical tool in reservoir characterization: Scandinavian Oil-Gas Magazine, NO. 5/6.

--------, 2007, Advanced techniques for simultaneous AVO inversion (RockTrace, RockMod): CSPG CSEG Convention, p. 115-116

Pendrel, J., and Van Riel P., 1997, Methodology for seismic inversion and modeling: A western Canadian reef example: CSEG Recorder, 22, 5-15.

Pendrel, J., Debeye, H., Pederson, T. R., Goodway, B., Dufour, J., Bogaards, M., Stewart, R. 2000, Estimation and interpretation of P and S impedance volumes from simultaneous inversion of P-wave offset data, CSEG Ann. Mtg. Abs. paper AVO 25.

Pennington W. D., Ibrahim, M., Turpening, R.M., Trisch, S.P., Richardson, J.P., Asiala, C., and Mabrouk, W. (2008), Crosswell Seismic Amplitude-Versus-Offset for Detailed Imaging of Facies and Fluid Distribution within Carbonate Oil Reservoirs, report to Dept of Energy, available at www.osti.gov/servlets/purl/946424-Mm7X2f/.

Pray, L.C. (1958), Fenestrate Bryozoan Core Facies, Mississippian Bioherms southwest United States, Jour. Sed. Petrology, Vol. 28

Rector J.W., Lazaratos S. K., Harris J. M. and Schaacks M.V., 1995, High-resolution crosswell imaging of a west Texas carbonate reservoir: Part 3-Wavefield separation of reflections: Geophysics, 60, P.692-701

Sams, M. and Saussus, D., 2007, Estimating uncertainty in reserves from deterministic inversion. Petroleum Geostatistics 2007 - Cascais, Portugal, Extended Abstracts, P17.

Sams, M. and Saussus, D. (2008) Comparison of uncertainty estimates from deterministic and geostatistical inversion. SEG Technical Program Expanded Abstracts 2008: pp. 1486-1490.

Sengupta, M. and Bachrach, R., 2007, Uncertainty in seismic-based pay volume estimation: Analysis using rock physics and Bayesian statistics. The Leading Edge, February 2007, 184-189. 
Shafer, R.H. (1974), Silurian Reefs of Northern Indiana, Reef and Interreef Macrofaunas, Bull. Am. Assoc. of Petrol. Geol. Vol. 58, No. 6

Sharma, G. D., (1966), Geology of Peters Reef, St. Clair County, Michigan, Bull. Am. Assoc. of Petrol. Geol. Vol. 50, No. 2

Sheriff, R. E., Geldart, L. P., (1995), 2nd Edition. Exploration Seismology. Cambridge University Press.

Smith, B.A., Gallagher, J.G.,Hoover, G.M., and Hufford, J.M.,1993, Characteristics of crosswell wave propagation at varying well offsets, 55th mtg.: Eur. Assn. of Expl. Geophys., Session: C007

Tarantola Albert, 1984, Inversion of seismic reflection data in acoustic approximation: Geophysics, VOL. 49, NO. 8, P. 1259-1266.

------, 1986, A strategy for nonlinear elastic inversion of seismic reflection data: Geophysics, VOL. 51, NO. 10, P. 1893-1903.

Trisch, S.P. 2006, Crosswell Seismic Amplitude Variation with Angle Studies at a Niagaran Reef, MS Thesis, Michigan Technological University.

Wyllie, A.S., and Wood, J.R., 2005, Well-log tomography and 3-D imaging of core and log-curve amplitudes in a Niagaran reef, Belle River Mills field, St. Clair County, Michigan, United States, Bull. Amer. Assoc. Petroleum Geologists, 89, 409-433. 


\section{Geostatistical Inversion of Crosswell Seismic data over Michigan Reefs}

\subsection{Summary}

Inversion of seismic data for impedance has become a standard part of the workflow for quantitative reservoir characterization. Images of elastic properties obtained from both deterministic and geostatistical inversion of a high-resolution crosswell seismic survey were used to obtain the internal structure, porosity distribution and heterogeneity of Niagaran Michigan reefs.

High-resolution crosswell seismic surveys were conducted over a Silurian (Niagaran) reef at two fields in northern Michigan. One of these, Springdale, included two monitor wells that are located external to the reef, and the other, Coldspring, employed two production wells within the reef.

While deterministic inversion is designed to ignore uncertainty and yields only a single model of elastic properties, geostatistical inversion produces multiple models (realizations) of elastic properties, lithology and reservoir properties. These realizations can be combined to yield volumes showing the probability of certain lithologies and also can be used directly to quantify the uncertainty in any of the resulting model properties. The geostatistical inversion outputs volumes with greater detail than those obtained through deterministic inversion using seismic data alone.

The deterministic inversion over Coldspring showed the internal structure inside the reef. Because of the high incompressibility known for the carbonate rocks typical of reefs such as this, we attributed the impedance variations to lithology (including porosity) changes, rather than fluid changes. The geostatistical inversion showed greater details of the distribution of porosity inside the reef than did the deterministic inversion.

The fact that, while in Coldspring we have access to complete log suites within the reef proper, in Springdale such logs exist only outside the reef; this leads to an opportunity to approach lithologic interpretations at Springdale in two different approaches. The first approach uses the geostatistical inputs from Coldspring and applies them into Springdale geostatistical inversion. The result for the top of the Springdale reef is found to be in agreement with the known geology and with previous deterministic inversion; however, the middle and the base of the reef displayed porosities higher than those known from the one well through Springdale reef. 
The second approach is a straightforward geostatistical workflow using the well penetrating the reef with incomplete log suite. The shear sonic and density were synthesized using standard petrophysical evaluation and rock physics modeling techniques. The second approach provides insight into the internal geometry of this reef with higher details for lithology and porosity estimation. The final results match the geologic interpretation of the reef model quite well.

The inversion images for both fields provide the best views of Niagaran pinnacle reefs obtained to date. The tops of the reservoirs can be clearly distinguished. The porosity architectures internal to the reef are imaged with high detail; and the images provide additional insight into the internal geometry of this reef while providing data that should be useful for reservoir management.

\subsection{Introduction}

The details of the interior of oil and gas reservoirs are always unknown. The internal heterogeneity controls their productivity, and if that heterogeneity is not wellcharacterized, the reservoir engineer is unable to produce the field optimally. Seismic imaging of crosswell data that can reach frequencies up to $4 \mathrm{kHz}$ have the ability to provide a finely detailed image and permits the engineer to understand the heterogeneity of the reservoir.

Crosswell seismic data can be used significantly to improve knowledge of the vertical and lateral heterogeneity of the reservoirs, as pointed out by several investigators (Lazaratos, et al., 1991; Lazaratos, 1993; Rector et al., 1995; Pennington et al., 2008; Ibrahim, et al., $2009 \& 2010$ ). The piezoelectric source used in the acquisition can reach frequencies up to $4 \mathrm{kHz}$, enabling events on the order of 10 feet thick to be imaged clearly, if the velocity model is sufficiently detailed and accurate. Crosswell seismic data are acquired by placing seismic sources and receivers in wellbores close to the target of interest. The data set should contain a range of angles limited only by the geometry of the source and receiver configuration, the separation of the boreholes used and the depth to the target. However, the wide angles present in crosswell imaging provide additional information that is not present in typical surface-seismic acquisition, and this often includes the critical angle for reflections of interest.

This work employs two simultaneous AVO inversion techniques: deterministic and geostatistical. Deterministic simultaneous inversion is based on the constrained sparse spike inversion (CSSI) algorithm, extended to AVO domain, and provided by Fugro-Jason Inc. This inversion approach, through steps of iterations, estimates the optimal elastic parameters by minimizing an objective function containing multiple terms of misfit functions. These user-control misfit functions are designed to make 
the solution honor of the seismic data, to keep the sparsity criterion, and to constrain the results to known or inferred geology (Debeye and van Riel, 1990; Pendrel and Van Riel, 1997; Sam and Saussus, 2008).

Geostatistics is the study of phenomena that vary in space and/or time (Deutsch, 2002). Caers 2005 defined the geostatistics as the branch of statistical sciences that studies spatial/temporal phenomena and capitalizes on spatial relationships to model possible values of variable(s) at unobserved, unsampled locations. The acceptance of geostatistics that originated from oil and mining industries came through many successful applications to cases where decisions were required to be based on interpretations from sparse spatial data sets.

Geostatistical simultaneous AVO inversion as employed here brings together aspects of Bayesian Inference, Geostatistics and classic AVO inversion within a Markov Chain Monte Carlo (MCMC) framework (Pendrel, 2007). The method can incorporate well information formally or run in a blind-to-the-wells mode. Another fundamental feature of this geostatistical method is the possibility of imbedding facies or lithotype estimation formally within the inversion procedure. The output attributes of each voxel are then, P Impedance, S Impedance (or Vp/Vs), Density and Facies. Indeed, any other reservoir properties (eg. Porosity, Permeability, Sw) can be estimated simultaneously within the inversion procedure or in a follow-up runs (through a process called cosimulation).

The geostatistical method recognizes no single answer but rather a set of solutions, all belonging to a joint probability density function (pdf) determined by Bayesian Inference. The process involves using the MCMC technique to sample this pdf to find a fair set of possible outcomes, each consistent with the input seismic. From the variability of these outcomes (also called simulations or realizations), uncertainties and probabilities can be inferred. Because the algorithm works on a (multi-channel) voxel-by-voxel basis rather than trace-by-trace, remarkably few realizations are required to estimate probabilities; usually, ten to twenty are sufficient (Pendrel, 2007).

The properties of engineering interest, such as porosity, can be cosimulated from the elastic properties and the lithology (or they can be inverted directly within the inversion processes). The output of this process is multiple models (realizations) of porosity (or other) volumes. These are in turn combined into maps of mean, minimum, maximum and standard deviation of that property.

Springdale and Coldspring are two oil fields each consisting of only one pinnacle reef, in northern Michigan (figure 6.1), where the crosswell seismic data were acquired and processed in 2005-2006 over pinnacle reefs for the purpose of this research. Both reservoirs have been produced for many years, and although they originally contained exclusively oil (and connate water), they are now also strongly gas-saturated, and produce gas and water in addition to oil. Both reservoirs are 
essentially volumetric drive, with minimal water encroachment, if any. Thus, they have been drawn down to very low pressures: 25-50psi (172-345kPa) at Springdale, and about 1000psi $(7000 \mathrm{kPa})$ at Coldspring.

The boreholes conditions at the two fields are different. The Springdale field is a dedicated research facility where the boreholes were drilled specifically for research purpose. They are external to the reef and were drilled much deeper than the reef. The boreholes at Coldspring oil field are environmental production holes drilled into the reef.

The two fields were surveyed under different conditions, which led to different observations of signal-to-noise ratios. Springdale was observed from dedicated monitor wells that had no fluid flow, and the conditions were very quiet. At Coldspring, fluid flow through perforations in the receiver well led to tube-wave noise that was high amplitude and poorly organized, strongly affecting the quality of the raw data. After processing, the decrease in signal-to-noise ratio as distance increased away from the source well is apparent (figure 6.2).

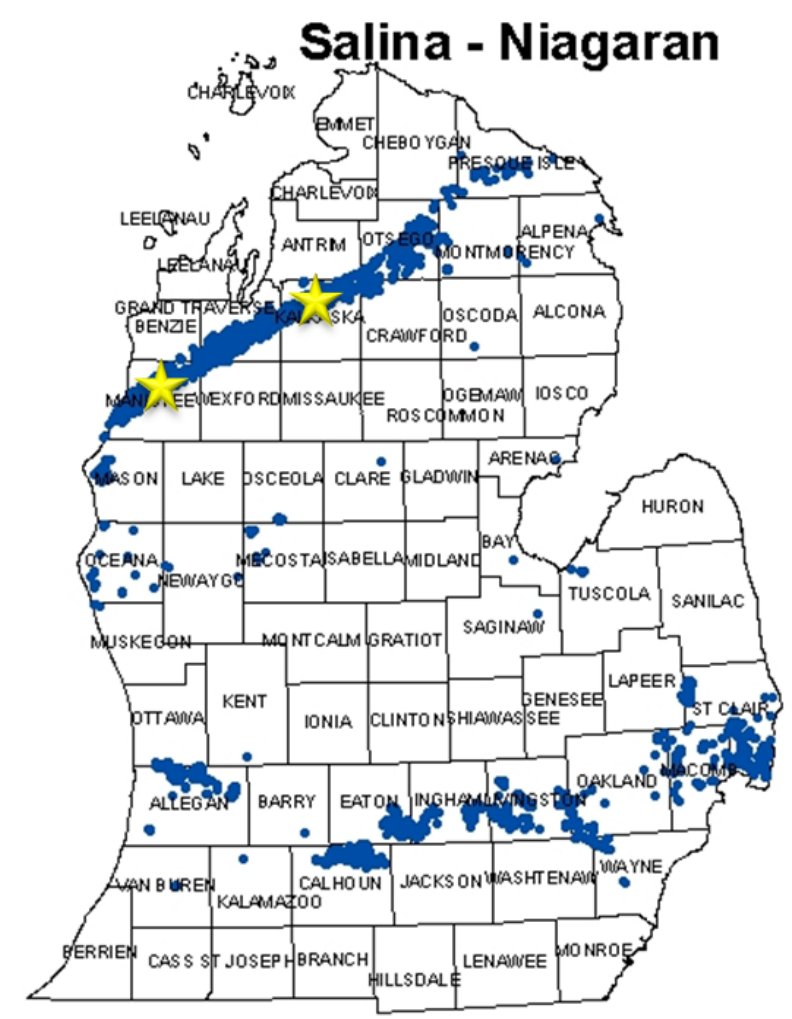

Figure 6.1: Niagaran reef locations in Michigan's lower peninsula. The two sites chosen for this study are indicated approximately by the star symbols. Base map is modified from the Michigan Dept. of Environmental Quality. (http://www.michigan.gov/images/FrmtnSalina-Niagaran_163193_7.jpg) 


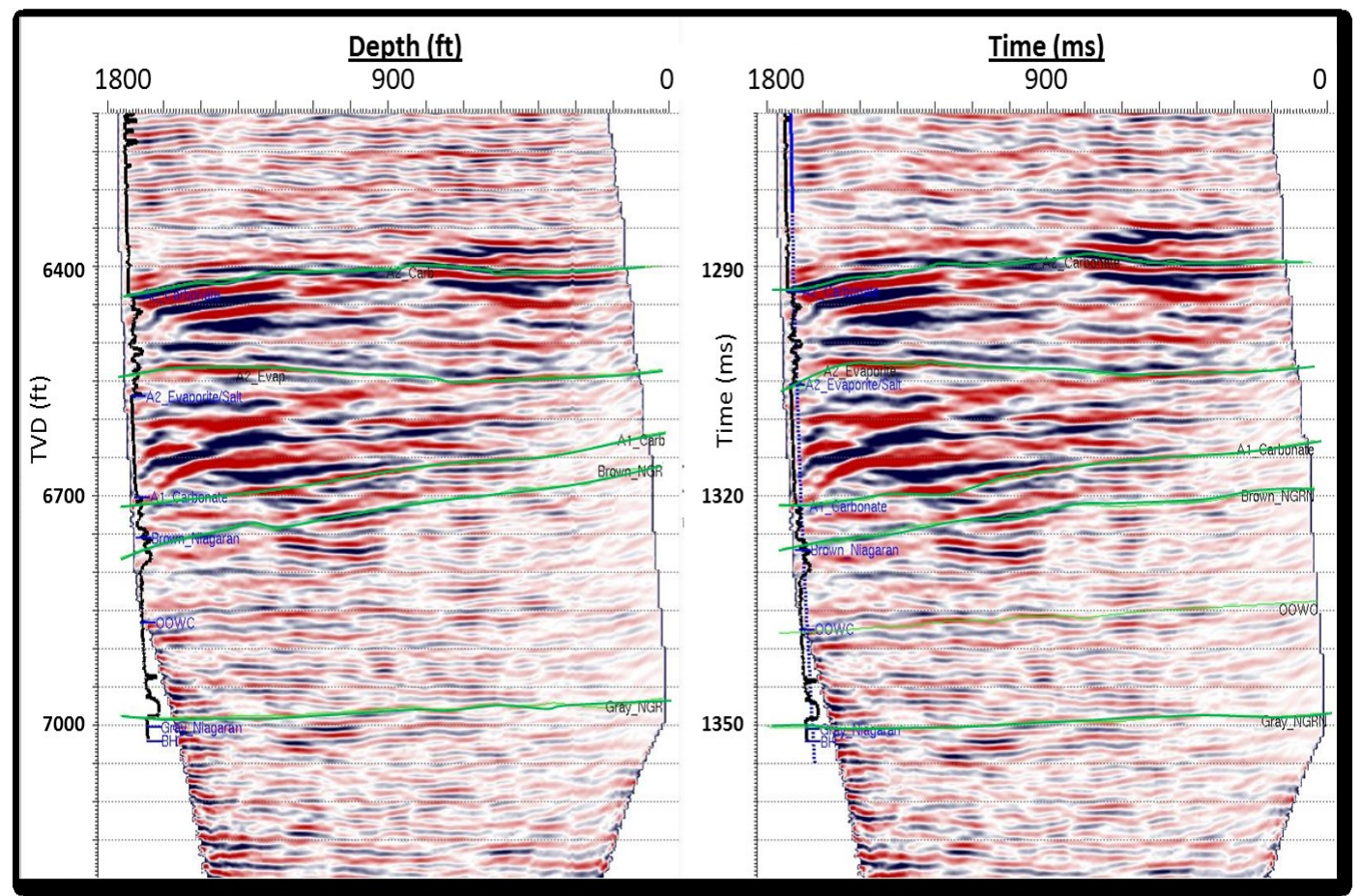

Figure 6.2: Seismic angle stacks over Coldspring area; data in Depth (left) and Time (right). Seismic horizons are in green; from top to bottom is A-2 carbonate, A-2 evaporite/salt, A-1 carbonate, Brown Niagara and Gray Niagara. Fluid flow through the perforations in the receiver well (on the right side) led to tube-wave noise that in turn led to a decrease in signal-to-noise ratio with increasing distance from the source well (on the left side). The source well (Coldspring 1-29) is shown to the left with Gamma Ray log in black.

The Niagaran pinnacle reefs were formed during a period of cyclic carbonateevaporite deposition in latest Niagaran-early Salina Time. High-sea-level phases are equated to carbonate depositions (Niagara, A-1 carbonate, A-2 carbonate) and lowsea-level phases are equated to evaporate deposition (A-1 evaporite, A-2 evaporite and B evaporate) (Mesolella et al., 1974). Later, the reef and surrounding carbonates were dolomitized. The surrounding A-1 and A-2 carbonates were not uniformly affected where the dolomitization increases toward the reef and decreases away from the reef (Jodry 1969). Gill 1977, introduced a reef model that is divided into three stages or rock types: the top is supratidal (stromatolite), the middle is organic reef (boundstone), and the bottom is biohermal stage (wackestone).

Elastic properties images obtained from such high-frequency seismic crosswell data improve the ability to understand the internal architecture of the reservoir in terms of facies, and consequently increases confidence in decisions, enabling a robust evaluation for maximizing the reservoir recovery. 


\subsection{Deterministic and Geostatisitical inversion at Coldspring}

In the last two decades, inversion of seismic data for impedance has become a standard part of the workflow for quantitative reservoir characterization. Inversion makes use of multiple volumes of seismic data, well logs, seismic velocities (optionally), and interpreted horizons to generate volumes of elastic properties (Pimpedance, S-impedance and density). Elastic properties can be related to petrophysical parameters from wells (porosity, water saturation, shaliness, permeability, and others) and calibrated through a petro-elastic model. Given the elastic volumes from inversion, the reservoir properties can be estimated at any point within the survey.

The first step in the simultaneous AVO inversion workflow is the creation of partial stacks of seismic data, based on angle of incidence. The number of partial stacks recommended for inversion is always a compromise among the stability of inversion parameters, signal-to-noise ratio and wavelets. For stabilizing the inversion parameters and hence the results, we would like to use as many partial stacks as possible; however, for stability of signal-to-noise ratio and wavelets we need to limit the number of stacks. In case of good quality seismic data (high signal-to-noise ratio), the creation of many partial stacks to stabilize the inversion parameters and keep a reasonable level of signal-to-noise ratio per stack is possible and easier than the seismic data that of poor quality (low signal-to-noise ratio).

For Coldspring data, the decrease in signal-to-noise ratio with increasing distance from the source well (figure 6.2) limited the process to only three angle stacks, and not the usual five required to obtain density, in spite of the wide angles present in the crosswell data set.

Deterministic inversions are designed to ignore uncertainty. At each step within a deterministic inversion workflow where uncertainty exists, the most likely solution is usually adopted. Deterministic inversions are typically constrained by a low frequency model and a sparsity criterion. The low frequency model is needed to provide information at wavelengths below the seismic bandwidth. In Coldspring, the low frequency model was derived from the logs of both wells (Coldspring 1-29 and 429). Figures 6.3 and 6.4 show the results of simultaneous deterministic inversion (Pimpedance and S-impedance). 


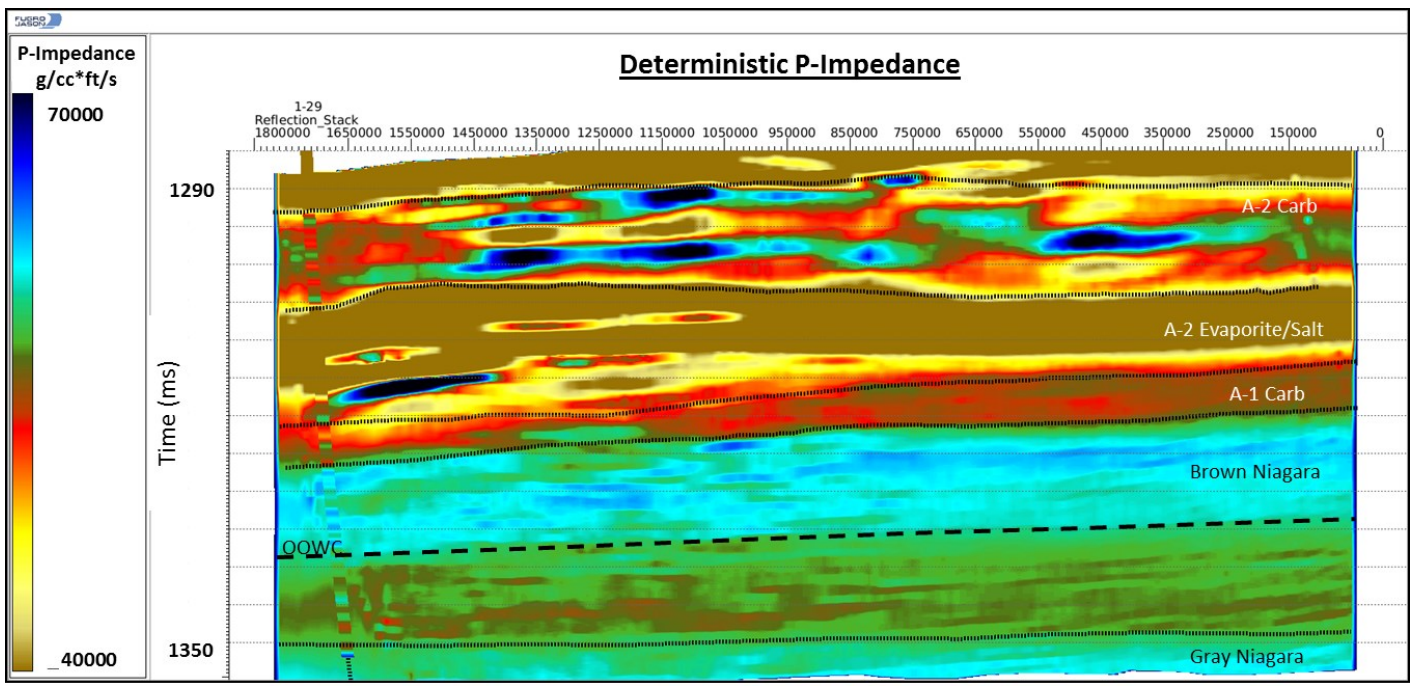

Figure 6.3: P-Impedance results from deterministic inversion. The horizons are shown in black (fine dashed lines). The thick dashed line represents the Original-OilWater-Contact (OOWC), which was picked after inversion and not used as an input to the earth model. The correlation between the high-cut well log P-impedance (700 $\mathrm{Hz}$ ) and the full band inversion results show a good match.

The primary objective of many AVO/AVA studies is to measure the change in $\mathrm{Vp} / \mathrm{Vs}$, the ratio of P-wave velocity (Vp) to S-wave velocity (Vs), which is usually dominated by changes in the $\mathrm{Vp}$ term, which in some clastics may indicate a change in fluid. $\mathrm{Vp}$ is only loosely dependent on fluid content in carbonates, where the rock frame comprises a larger proportion of the system's incompressibility than in poorly consolidated clastic rocks. In the carbonate environments, same of this study, the reefs are poorly suited for direct detection of hydrocarbons because of the lowporosity carbonate comprising the formation are highly incompressible with only a tiny volume fraction potentially occupied by hydrocarbons, and their velocities are therefore very weakly dependent on fluid content.

The change in impedances noticed in the inversion results (figures 6.3 and 6.4) coincides roughly with the original oil-water contact as interpreted by the well operators. We interpreted this change in impedance as a lithologic boundary where the lower-porosity reef material (containing the hydrocarbon reservoir) overlies higher-porosity reef material, typical of Michigan reefs. 


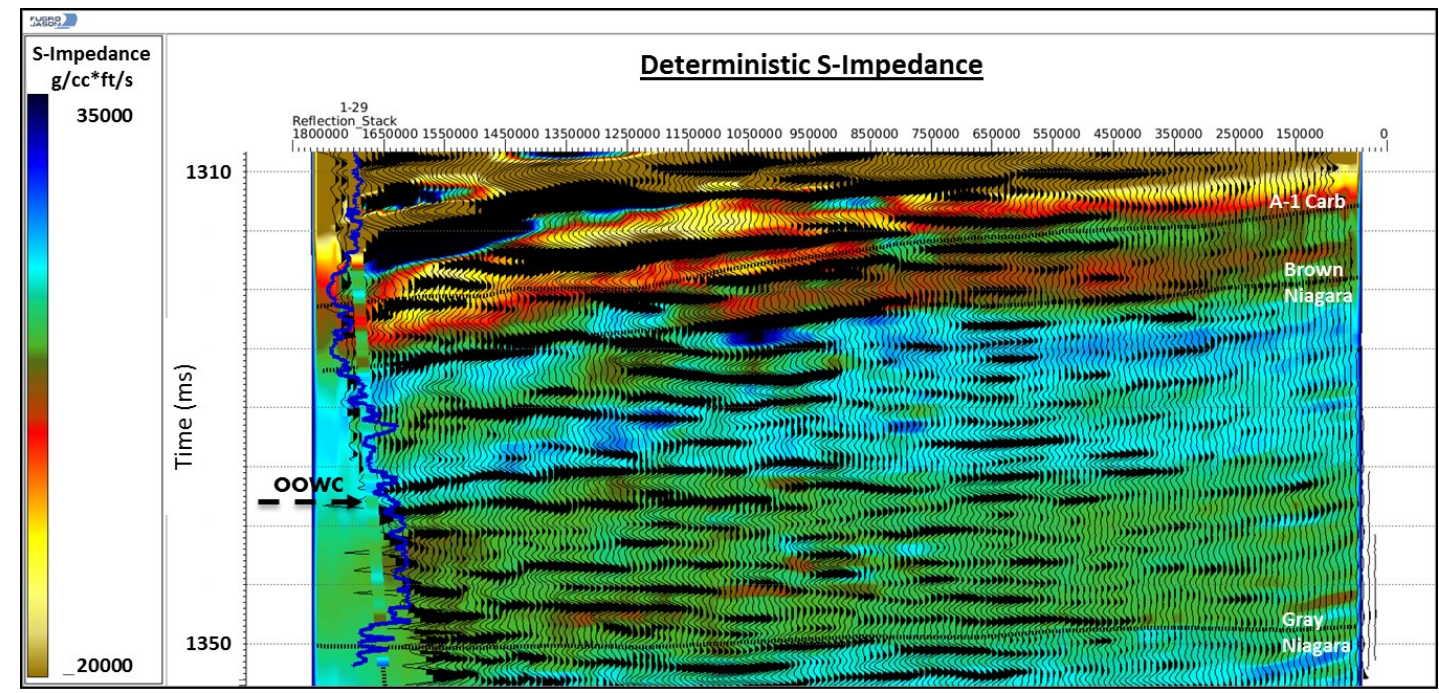

Figure 6.4: Deterministic inversion of S-Impedance results at Coldspring overlaid with seismic section (black wiggle traces). Neutron porosity well log shown in blue. Negative amplitude reflectivity can be seen on seismic where the black arrow points out the location of the OOWC. The change in impedance noticed on seismic labeled as $O O W C$ ) coincides with increasing porosity indicated by the neutron porosity log, suggesting that the inversion results provide lithology, not fluid indications.

Tuning as a function of the bed thickness is well known. Despite the high frequency content of crosswell seismic data, there is still a chance that the thin layers within reservoirs falls below the tuning thickness. In addition and because of the sparsity criterion (reflectivity sequences composed of fewer, higher-amplitude isolated spikes are more favored over those composed of many), the sparse spike inversion will always tend to select thicker solutions over detailed ones. Generally speaking, it will be always hard for deterministic inversion approaches to solve for reservoir properties as well as the thickness of a thin body at the same time. Geostatistical approaches can offer assistance in such cases.

The geostatistical approach produces inversion results with far more detail than deterministic inversion. It jointly models the lithology and the elastic properties, instead of consecutively as in deterministic inversion, thus leveraging synergies. The magnitude of difference in details between geostatistical and deterministic inversion becomes increasingly pronounced for thinner reservoirs, particularly those beyond the vertical resolution of typical seismic interpretation.

Bayesian Inference is a probabilistic inference method used for systematically incorporating various sources of uncertain information (beliefs) together in an unbiased and consistent manner. These beliefs can take a variety of forms including variogram ranges, lithotype probabilities, multivariate probability density functions (pdfs), wavelets and seismic angle stacks. Taken individually, these beliefs do not 
provide enough information to generate highly detailed and realistic model of the reservoir (Jarvis et al., 2008). A Markov Chain Monte Carlo then can be used for sampling the distributions to obtain multiple realization of reservoir.

Because geostatistical inversion is a model-based inversion, building the geological model is the first step in the process of minimizing uncertainty. Modeling the statistical characteristics of existing well data and then executing the inversion in an iterative manner to optimize all parameters should complete the workflow. Running deterministic inversion before the geostatistical inversion always offers a chance of minimizing some uncertainty by choosing a highly appropriate initial starting model for geostatistical inversion.

The deterministic results (figures 6.3 and 6.4) show that the Brown Niagara layer (reef) is divided into two major zones: an upper zone of lower porosity (above the OOWC horizon) and a lower zone (below the OOWC horizon) of higher porosity. For geostatistical inversion, this variation in porosity inside the reef should be taken into consideration by using a geological model of two layers. Another benefit of using this model is to avoid having a probability density function with bimodal distribution (continuous probability distribution with two different modes). Because the impedance is a layer property, picking the OOWC horizon to use it in the geological model was easier using the inversion results than using the seismic amplitude data.

Given that geostatistical inversion is more time consuming than deterministic inversion and that the lithology (facies) discrimination above the OOWC horizon is a major source of uncertainty in modeling this reservoir under study. Two lithology types (facies) are derived from the existing well control in the target interval, above the OOWC horizon: "tight" and "porous" carbonate (figure 6.5), with a value of 5\% used as the cutoff.

One of the key beliefs is comprised of the multivariate elastic-property pdfs where the crossplots of all properties observed for each lithology type are believed to arise from petrophysical considerations. In figure 6.6 the pdfs for tight and porous carbonates are shown. Good agreement can be seen between the sampled and the modeled pdfs. 


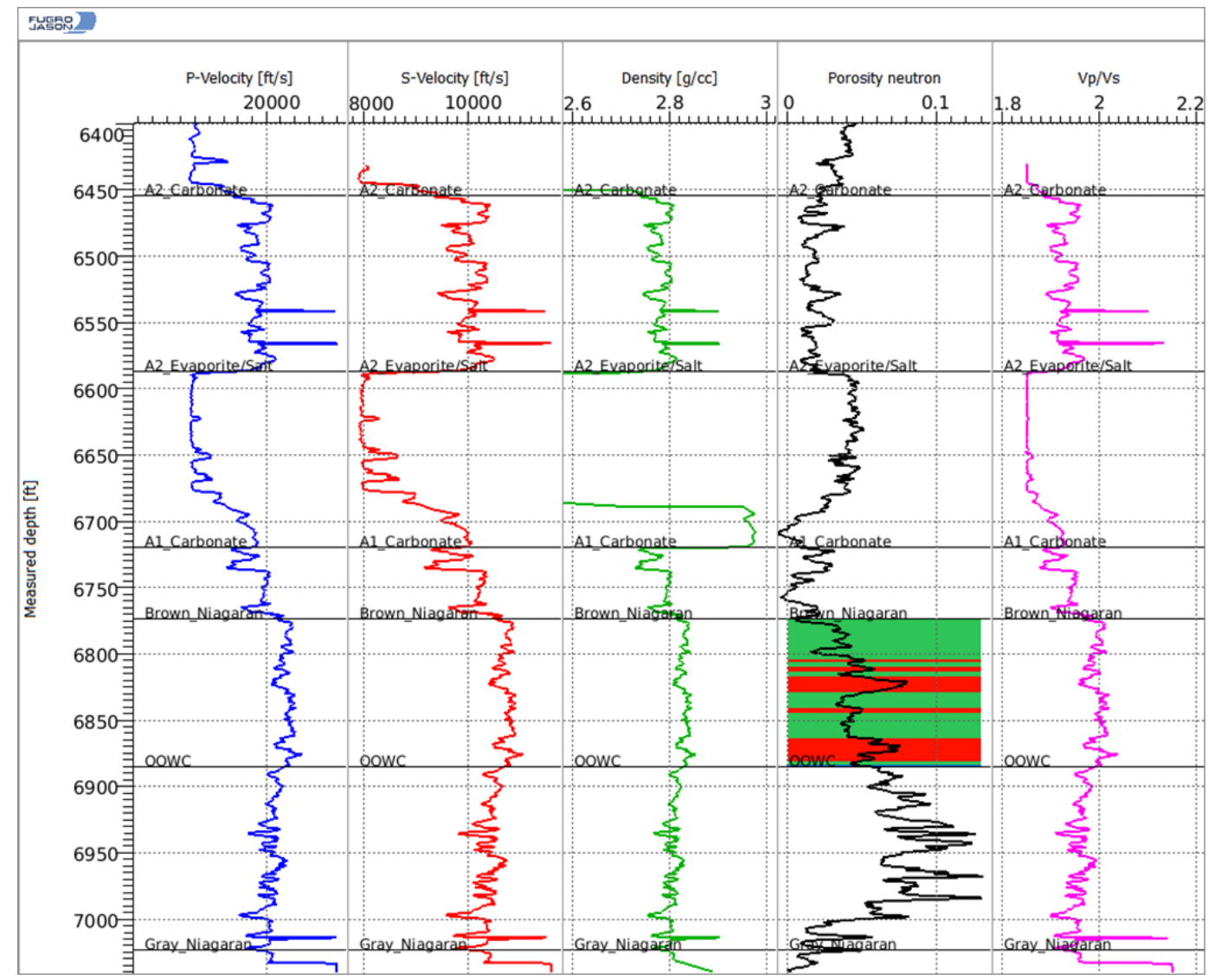

Figure 6.5: Log view for well Coldspring 1-29. Panels show well logs from left to right; p-velocity (blue), s-velocity (red), density (green), neutron porosity NPHI (black) and Lithology log (background color), and $V p / V s$ ratio (magenta). The lithology type log created base on cutoff; Tight Carbonate Type (green) $=$ NPHI < 0.05 and Porous Carbonate Type (red) $=N P H I>=0.05$ 

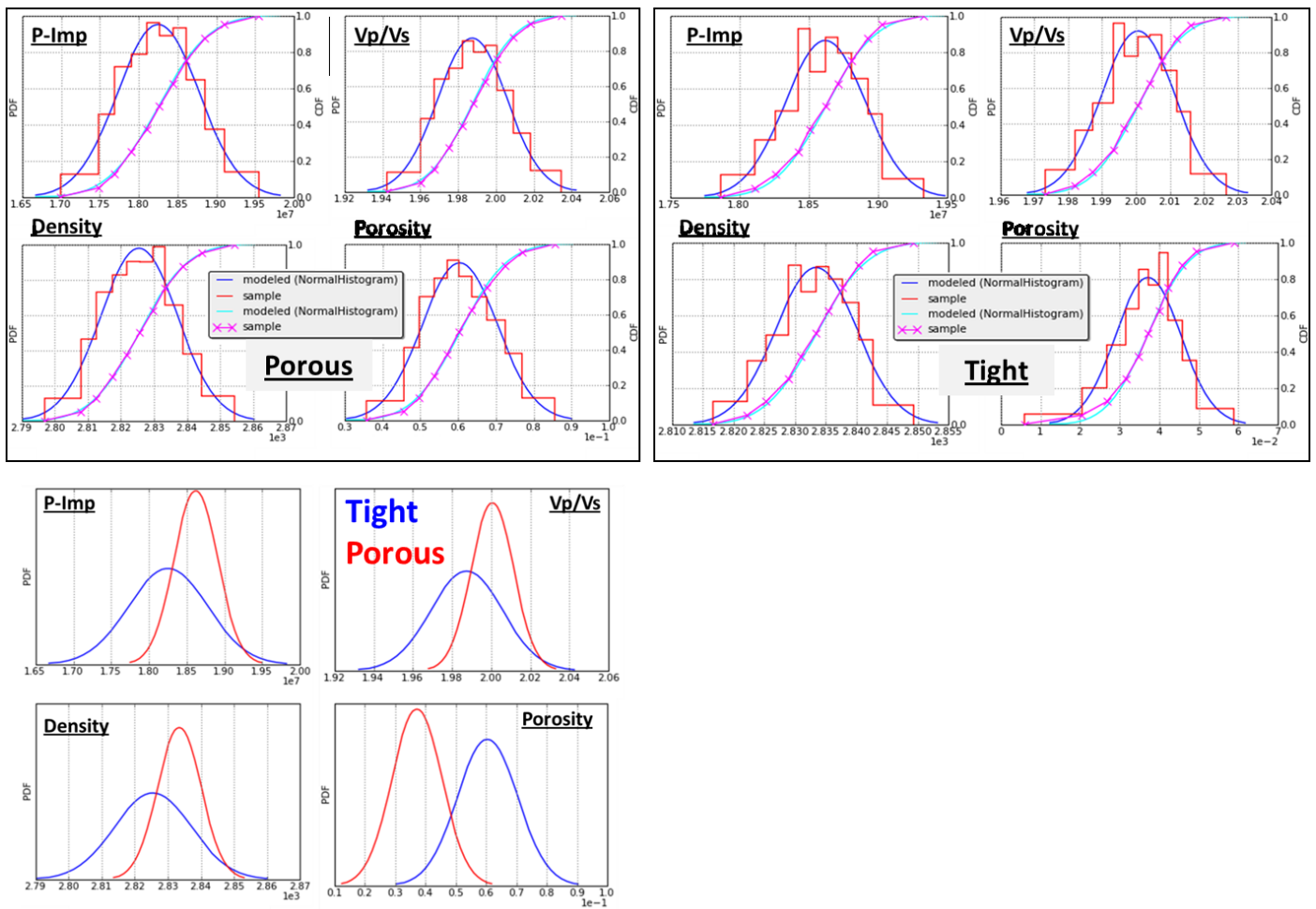

Figure 6.6: The probability density function (pdfs) for the lithology above the OOWC horizon. a) pdfs for porous carbonate for all elastic properties and porosity b) $p d f s$ for tight carbonate all elastic properties and porosity. Red and blue curves are the sample (from well log) and modeled (normal distribution) pdf while magenta and cyan are the sample and modeled cdf (cumulative density function). Good agreement can be seen between the sampled and the modeled pdfs and c) The relationship between porous and tight carbonate for the layer above OOWC.

Multiple sets of elastic properties and lithology (tight and porous carbonates) comprise the outputs of the inversion. The multiple model realizations provide a means of quantifying the non-uniqueness and uncertainty in the inversion result. Examples of two realizations for $\mathrm{p}$-impedance and $\mathrm{Vp} / \mathrm{Vs}$ are shown in figure 6.7. Each individual realization matches all available data and is an equally probable model; together, the set of individual realizations can be used to analyze the probabilistic distribution of model possibilities. The mean shown, in figure 6.8 , is the "average" estimate and is based on ten multiple realizations. 


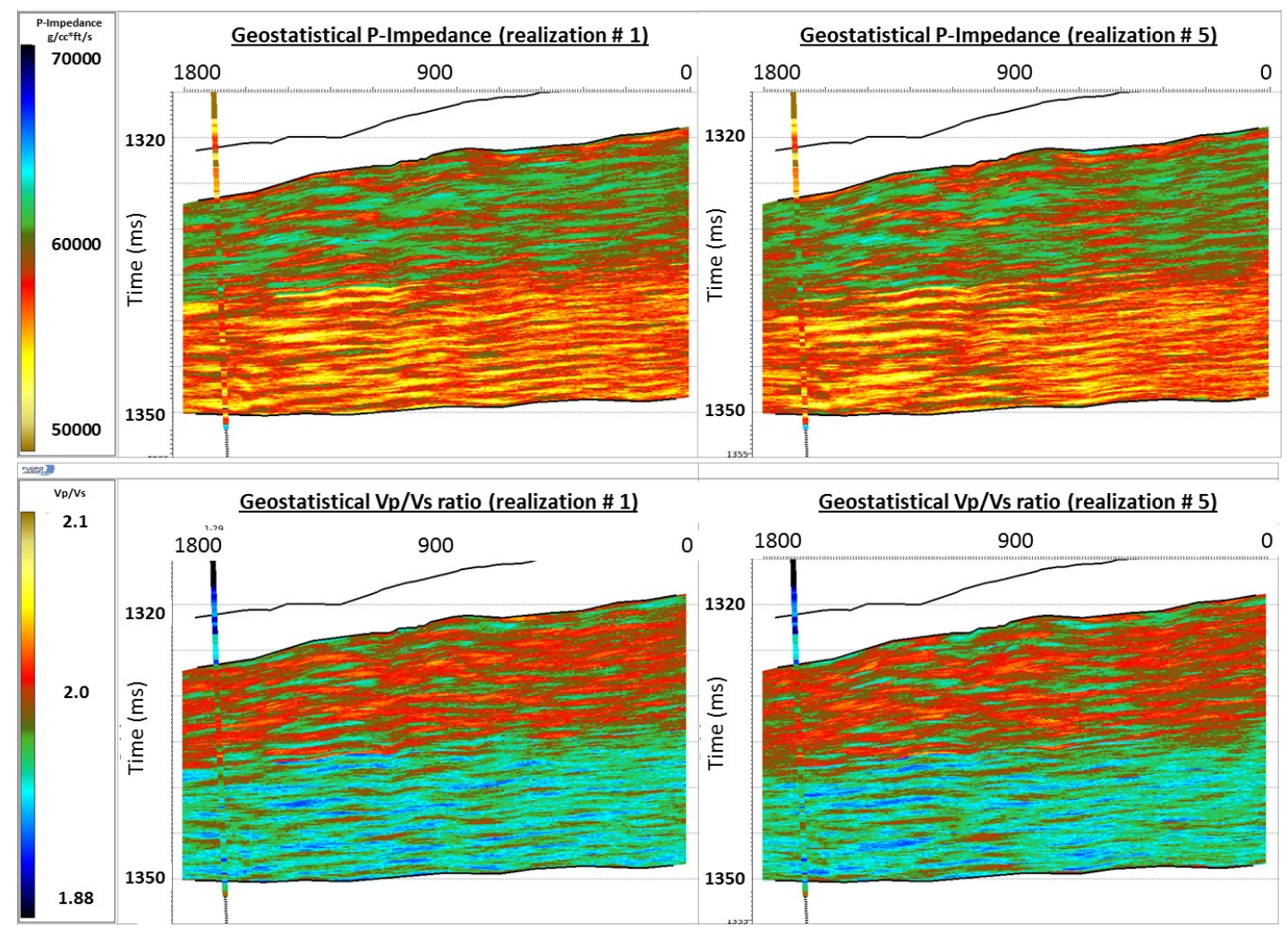

Figure 6.7: the results of two separate realizations for P-Impedance and Vp/Vs ratio from the geostatisitical AVO Inversion. The two realizations have slight differences which are mostly averaged out in the mean results.

Uncertainty assessment of the elastic properties was conducted by calculating the standard deviation of the ten realizations (figure 6.8). The standard deviation shows how much variation or dispersion exists from the average or the mean value. The uncertainty monotonically increases away from well locations. However, the small standard deviation of $\mathrm{P}$-Impedance and $\mathrm{Vp} / \mathrm{Vs}$ ratio (figure 6.8) confirms that the spatial range of uncertainty is low even away of the well location. Also, it confirms that the outputs are consistent with the estimated inputs of lateral distribution of carbonates. In figure 6.9 , the variability between ten realizations was used to determine probabilities for porous carbonate. A good correlation to the neutron porosity well log is evident. 


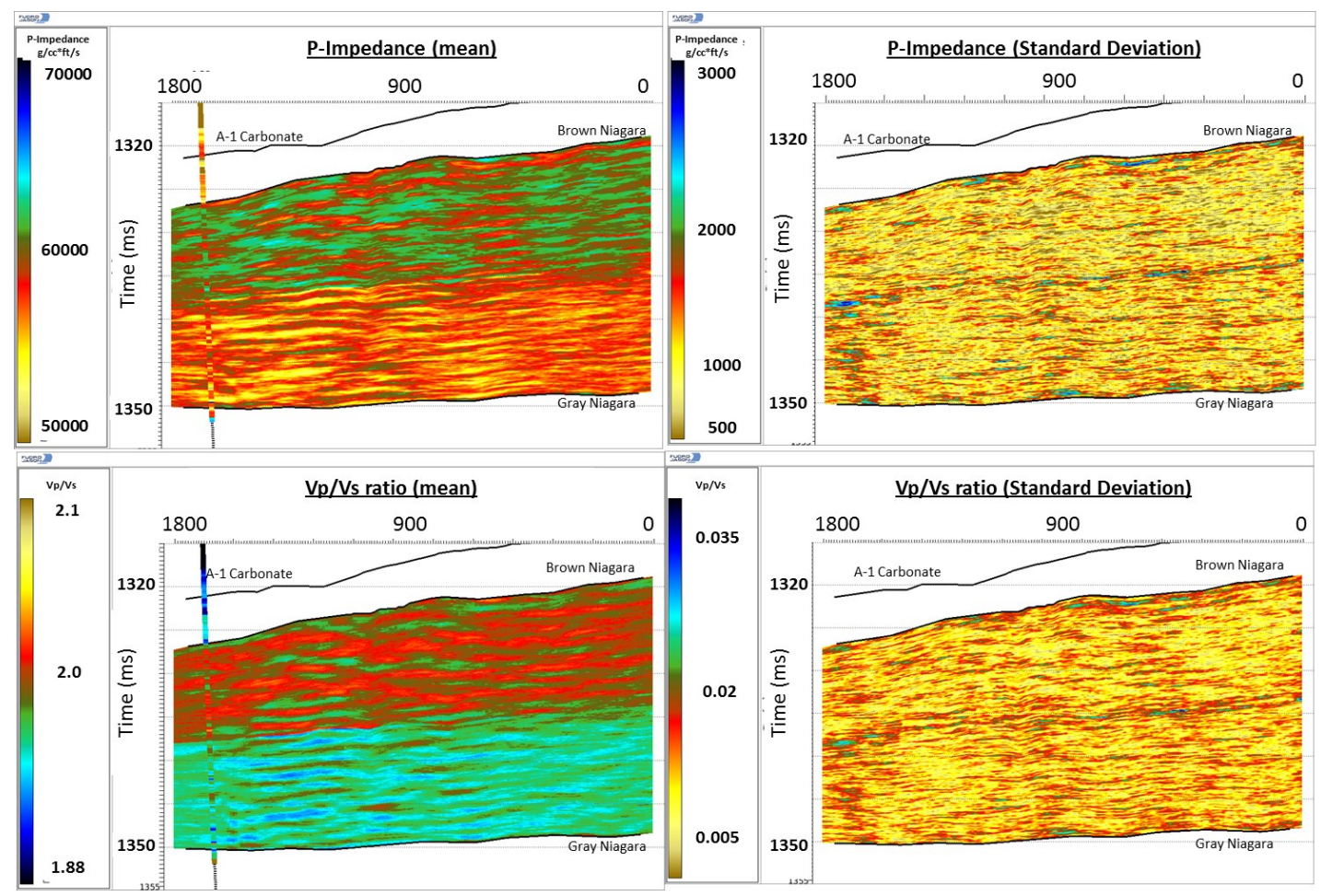

Figure 6.8: The results of mean P-Impedance combined from ten realizations and its standard deviation (up); mean $\mathrm{Vp} / \mathrm{Vs}$ ratio and its standard deviation (lower). Although the results were run on blind-to-well mode (unconstrained to the well log) the correlations of $P$-impedance and $V p / V s$ well logs with the inversion results are good. The yellow color in the standard deviation figures represents low uncertainty (low standard deviation values). 


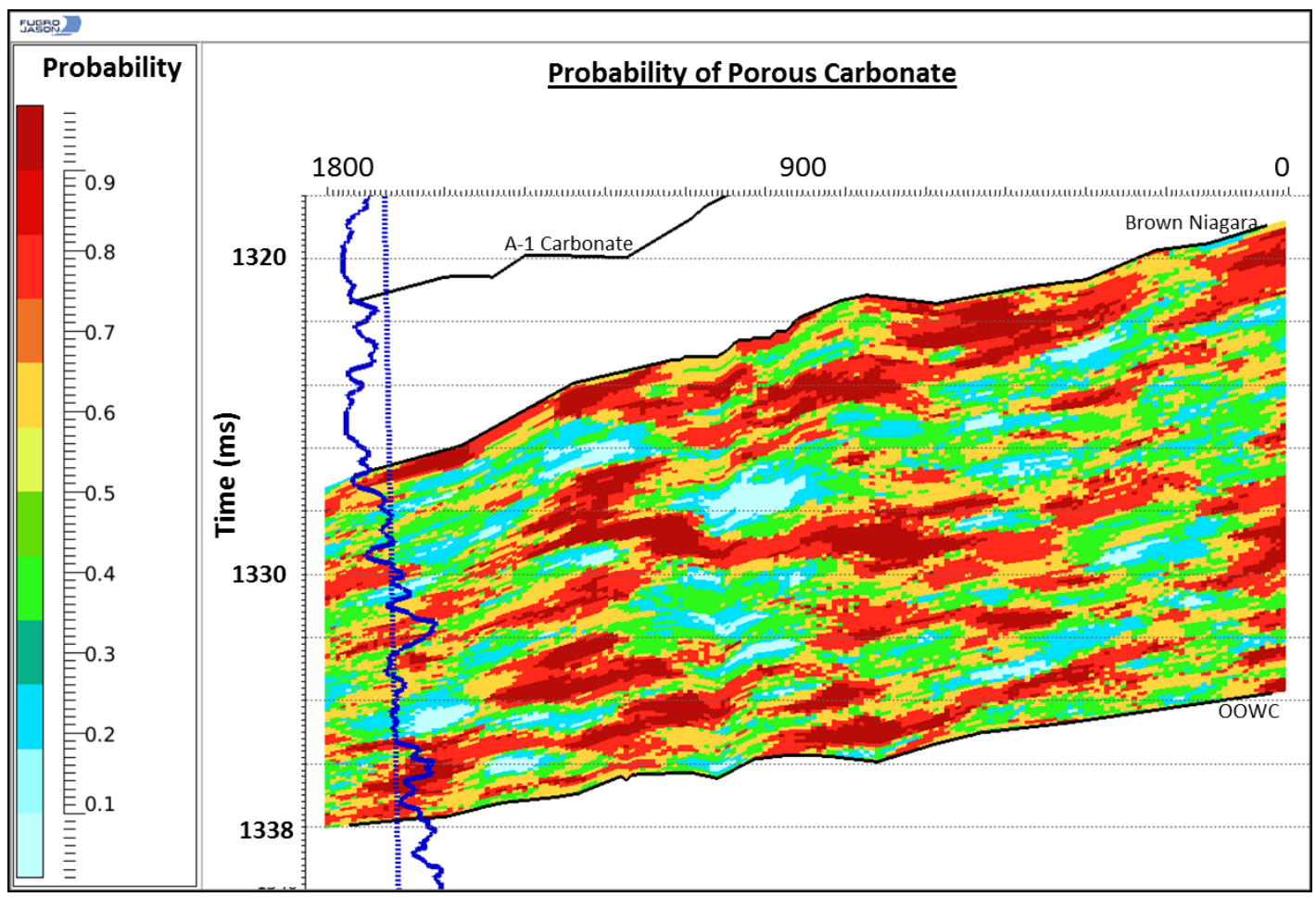

Figure 6.9: The variability between ten realizations was used to determine probabilities for porous carbonate. Good correlation between the neutron porosity well log, shown in blue, and the probability of porous carbonate is evident. The light to dark red colors represent the porous carbonate (porosity $>0.05$ ) with a probability of existence equal to or higher than $65 \%$.

Of all the attributes, p-impedance is most tightly coupled to porosity, and is often a key to reservoir characterization. The typical approach for generating porosity distribution from acoustic impedance involves establishing a correlation between the porosity and p-impedance well logs to create the porosity volumes from the results of deterministic inversion. The geostatistical inversion that solves simultaneously for the elastic properties and lithology allowing for inter-dependent correlation should give a better porosity results than those from deterministic inversion. In addition, each lithology effectively masks the other lithologies, enforcing a better correlation with porosity. The run for cosimulating the porosity can incorporate well information formally or run in a blind-to-the-wells mode. To get the most accurate porosity distribution and to prevent outliers, the cosimulated porosity was constrained to the well $\log$ and the mean porosity of ten realizations, shown in figure 6.10. 


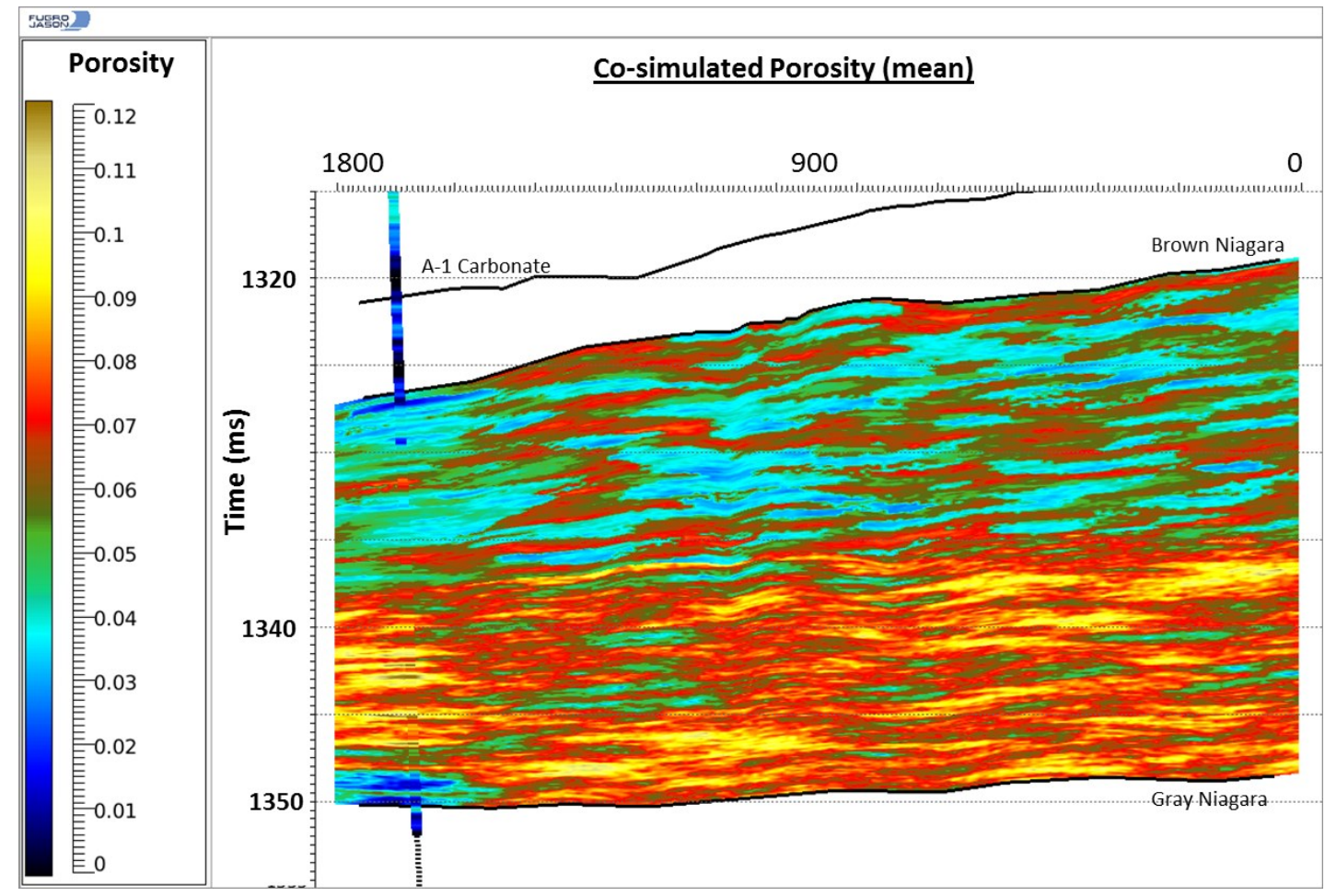

Figure 6.10: The neutron porosity well log is overlaid the cosimulated porosity results (well constrained).

\subsection{Geostatistical inversion at Springdale}

Inversion has proven extremely successful for reservoir delineation and characterization for two reasons. First, it reduces the tuning and interference effect of seismic wavelets and increases the bandwidth of the seismic data by adding the low frequency bands from well logs. Second, it transforms the seismic data from the interface properties to layer properties that are absolute values for the rocks themselves.

The main exploration problem for carbonate reef reservoirs often consists of determining the porosity distribution and evaluating the heterogeneity of the reservoir. Such an evaluation would improve the reservoir characterization and provide a number of reservoir development options. At Springdale reef, a comprehensive discussion using deterministic inversion over this field, to increase the internal details of the reef, was discussed in previous articles (Ibrahim et al., 2010 and 2012). 
However, one of the fundamental reasons to apply geostatistical inversion is to obtain greater resolution than can be obtained using seismic alone through deterministic inversion. So the main focus in the current paper is using geostatistical inversion to evaluate the vertical and lateral heterogeneity of the internal reef with finer detail and to assess the uncertainty associated.

Three well logs are essential for simultaneous AVO inversion (P-sonic, S-sonic and Density) as well as any lithology indicator log. The use of well logs in deterministic inversion is limited to the extraction of wavelets and the creation of the low frequency model; however, for geostatistical inversion the logs are used to estimate lithology probability, vertical variogram ranges, and the multivariate probability density functions (pdfs).

Both Springdale and Coldspring reefs belong the same trend of pinnacle reefs in Northern Michigan basin. This trend formed during a period of cyclic carbonateevaporite deposition in latest Niagaran to early Salina Time (Ordovician). The reefs and their surroundings are dolomitized. Springdale field has three wells; two located external the reef with full suites of logs and a third well which produces from the reef but has limited logs.

The uncertainty in the carbonate facies for reef reservoirs is usually higher than for carbonate platform reservoirs. This decreases dramatically if the reef is sampled by wells. Our motivation here was to study the use of geostatisitics established in a wellknown reef or nearby field and apply them to a reef with limited well information.

In this section we will present two approaches for geostatistical inversion at Springdale. The first method applies the inputs of geostatistics that had been derived and used in Coldspring; these inputs include two lithology types (tight/porous) using the $5 \%$ porosity cutoff, vertical and horizontal variogram ranges, and multivariate probability density functions (pdfs).

The second approach applies correlations from the single well that penetrates the Springdale reef. Because only the neutron-porosity log is available from among those required, the missing well logs are synthesized. At Springdale we make use of the data acquired from above the reflections and from beneath, and are able to use six partial angle stacks that together cover an angle range from $25^{\circ}-72^{0}$ (Ibrahim et al., 2012). This angle range and number of stacks should result in reasonable density results as well as $\mathrm{P}$ and $\mathrm{S}$ impedances.

Although the reefs at Coldspring and Springdale were formed during a similar deposition period, the two reefs are now found at different depths, with top of the reef at Coldspring at $6700 \mathrm{ft}$ and the top of the reef at Springdale at $4600 \mathrm{ft}$, In this case, the pdfs at Coldspring will be affected by the difference in depths and net confining pressures. Thus, to be able use theses pdfs in Springdale, we need to remove this trend from Coldspring's pdfs and estimate them for Springdale based on the residuals. 
Removing the depth trend can be done in various ways; applying a low-pass filter to the well logs to find the trend and then subtract form the original well logs to get the residuals. Since the velocity is always a good sensor for compaction, in this study, we removed the trend using the velocity field after scaling it into elastic properties (Pimpedance, S-impedance and Density), extract pseudo traces at the well location and then subtract these pseudo logs from the equivalent well logs. The outputs of this subtraction are the residuals logs of P-impedance, S-impedance and Density at the well location. We re-estimate the geostatistics based on the residuals at Coldspring, including the estimation of joint probability density functions (figure 6.11) and then we applied them into Springdale geostatistical workflow. The last step in this workflow, in order to get the full band inversion, is adding the trend back to the results. The trend at Springdale was estimated from the velocity field and added to the residuals inversion volume to get the full band inversion results (figures $6.12-$ 6.14).

The lithology probability volume output from inversion is non-unique and the results are mainly a function in the lithology types used in the multivariate probability density functions. Changing the cutoff values used to create the lithology type (or the number of types) will result in different lithology probabilities. In figures $6.12-6.14$, the inversion results using the geostatistics from Coldspring inversion yield two observations: first, the top of the reef is composed of tight carbonate rock; second, most of the rest of reef is porous (i.e., at least 5\% porosity). The first observation is in agreement with our geological knowledge about the internal structure of the reef, but the second observation is not as it should show less area of porous carbonate with zones of tight (or anhydrite plugged) carbonate as barriers. 

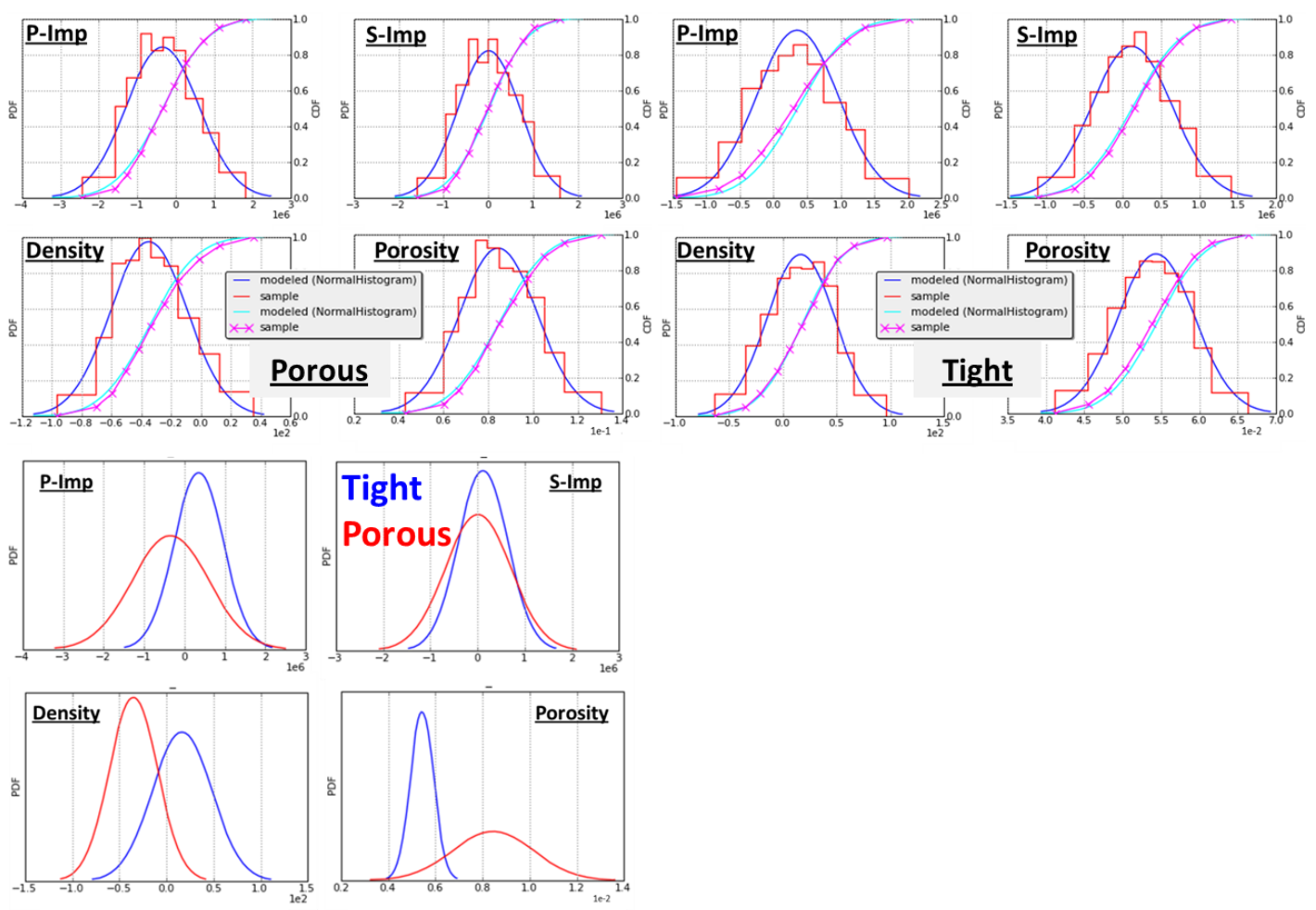

Figure 6.11: The Probability Density Function (PDFs) after removing the trend. Notice the $x$-axis value oscillate around zero (residuals). 


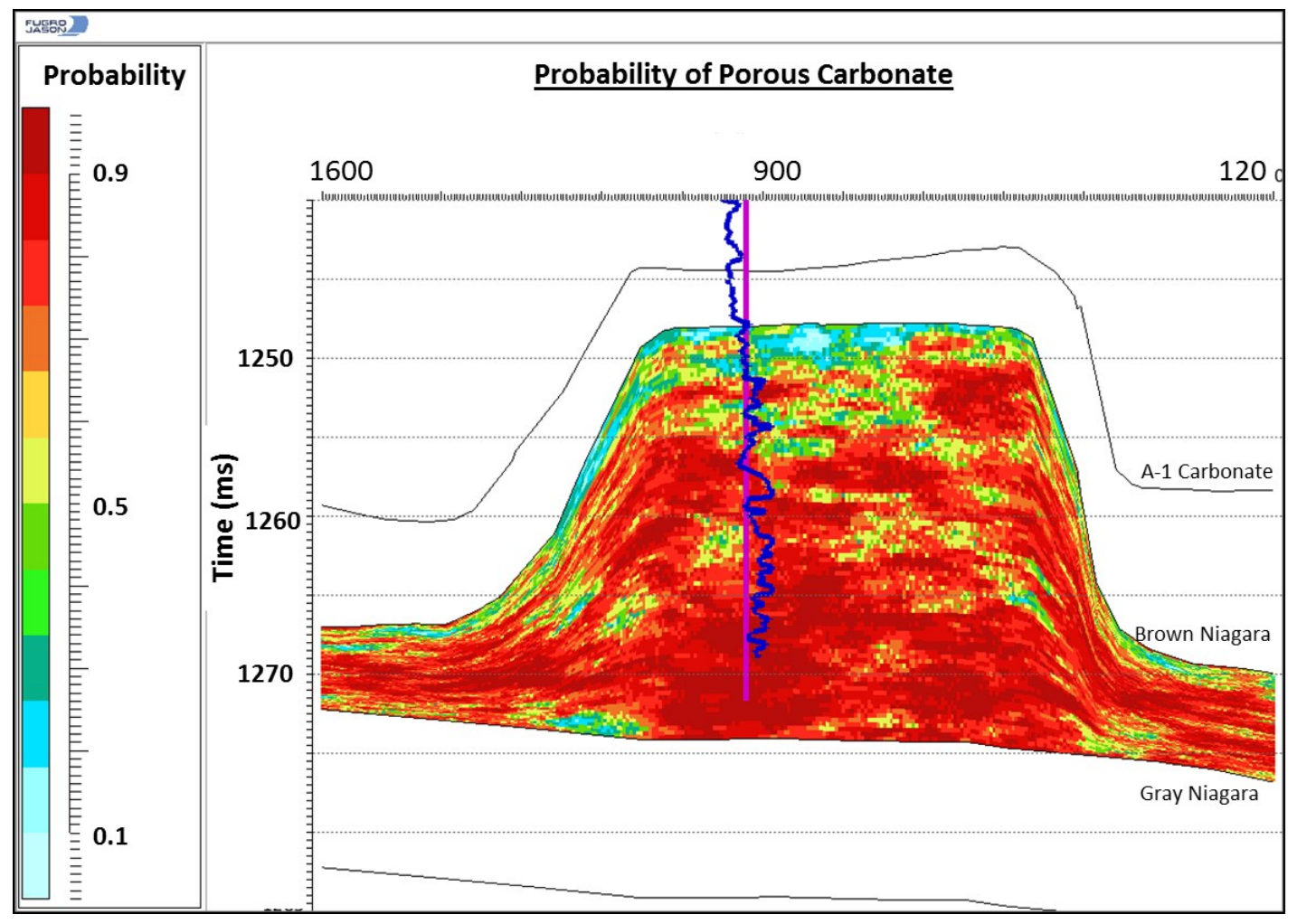

Figure 6.12: The variability between ten realizations was used to determine probabilities for porous carbonate. Neutron porosity Well log is shown in blue. The statistics for Coldspring was based on cutoff at 5\% porosity. The result shows almost the whole reef is porous, which is not particularly insightful.

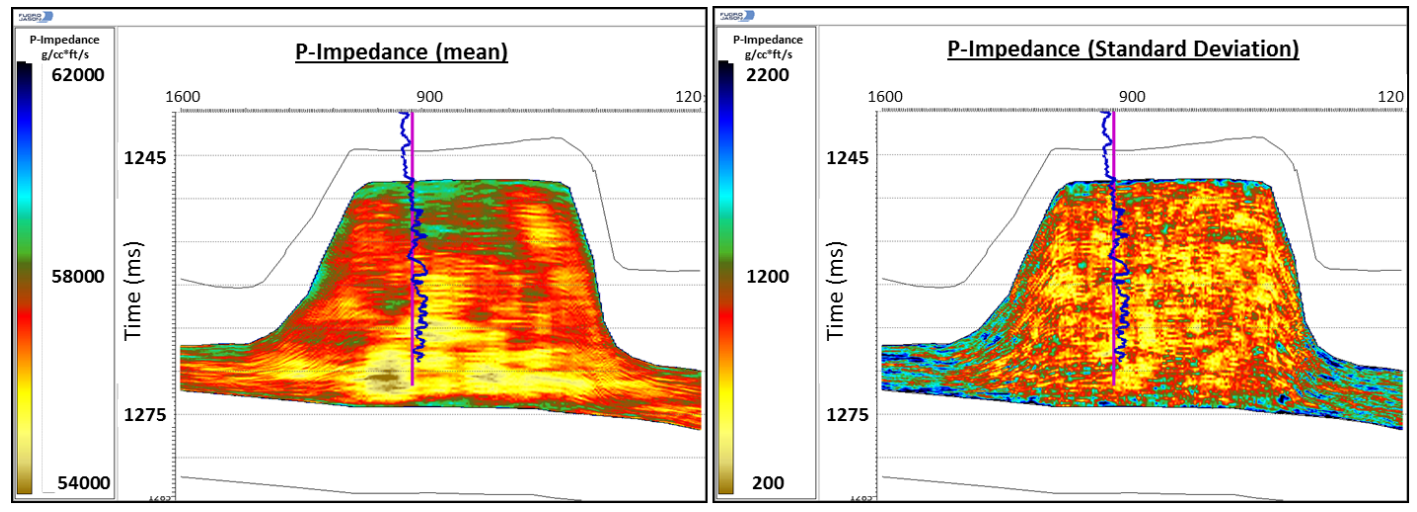

Figure 13: The results of mean P-Impedance combined from ten realizations and its standard deviation. 


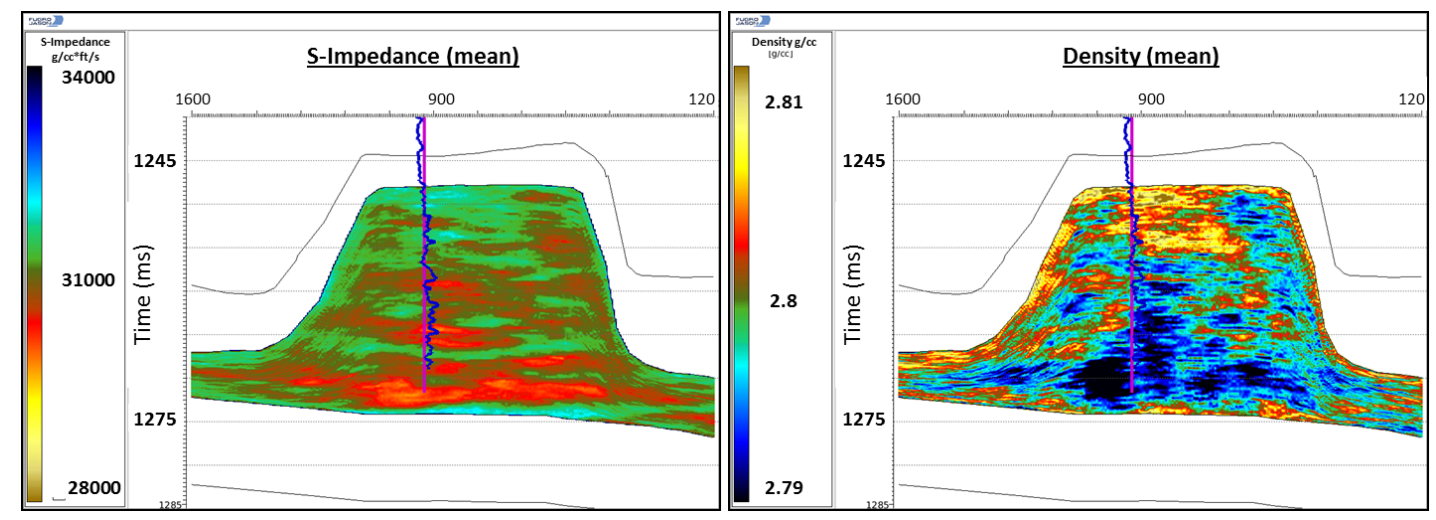

Figure 6.14: The results of mean S-Impedance and mean Density combined from ten realizations.

A comparison between the well logs at Springdale and Coldspring (figure 6.15) shows that the reef at Springdale has higher porosity values, especially above the OOWC horizon. Two points need to be mentioned here; first the geostatistics at Coldspring was derived from the upper part of the reef (above the OOWC horizon) but was used for the entire reef at Springdale. Secondly, the lithology type created for Coldspring was based on neutron porosity cutoff equal to $5 \%$ which fit the porosity distribution for this reef both above and below OOWC horizon. However, this 5\% cutoff does not seem to fit the interpretation of the data at Springdale. Figure 6.15 seems to suggest that the best cutoff that that fir the reef at Springdale is $7 \%$ not 5\% porosity. The joint probability distributions (pdfs) applied from Coldspring into Springdale considered the lithology probability between 5\% (Coldspring cutoffs) and $7 \%$ (the cutoffs proposed for Springdale) as porous carbonate and this resulted in showing the reef with more zones of porous carbonate.

The second approach is a straightforward workflow to run inversion using the well penetrating the reef. The shear sonic and density were synthesized using standard petrophysical evaluation and rock physics modeling techniques. Two lithology types created: porous and tight carbonate. 


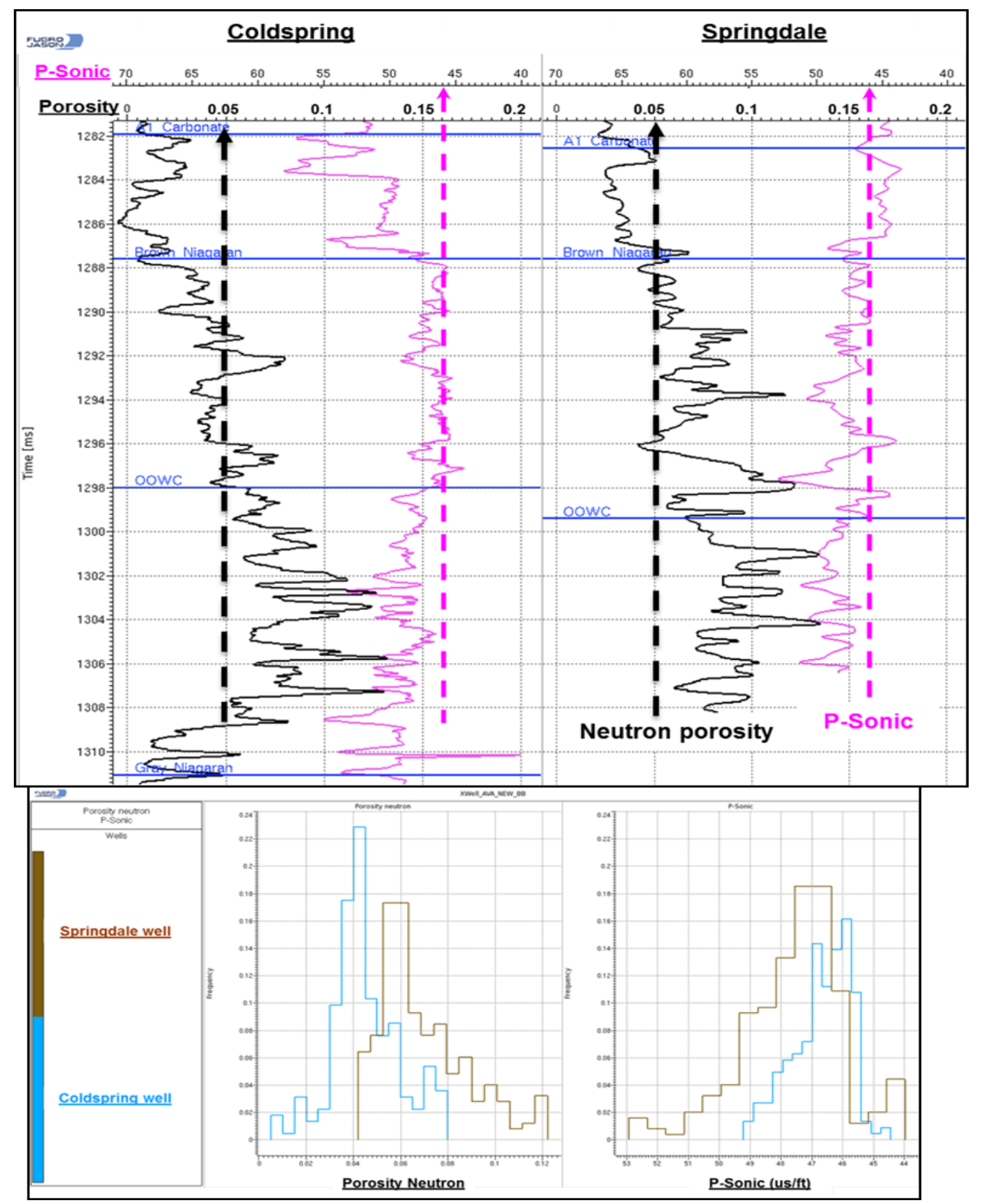

Figure 6.15: a) Neutron Porosity and P-Sonic log View: Coldspring (left) and Springdale (right). Well Logs flattened to the top of Brown Niagara. b) Histograms for P-Sonic and neutron porosity for Coldspring well (light blue) and Springdale (brown). A higher porosity in Springdale is evident 
Ten realizations are simulated, each consisting of volumes of lithology, P-impedance, S-impedance, density, and porosity at a sample interval of 100 us $(0.1 \mathrm{~ms})$. Each realization honors the rock physics distributions and relationships, the multiple seismic stacks and the geostatistical parameters to within a specified tolerance. The inversion was run on blind-to-well mode so the results are not constrained to well.

The lithology probability volume of ten realizations shown in figure 6.16 provides insight into the internal geometry of this reef with higher details compared to the deterministic inversion results, not discussed in this article. Barriers of tight carbonate are evident and the image matches the geologic interpretation of the reef model quite well.

Uncertainty assessment of the elastic properties was conducted by calculating the standard deviation of the ten realizations (figures $6.17-6.19$ ). The results of standard deviation confirm that the spatial range of uncertainty is consistent with the estimated lateral extent of carbonate units. Finally, the mean of ten realization of cosimulated porosity is shown in figure 6.20 .

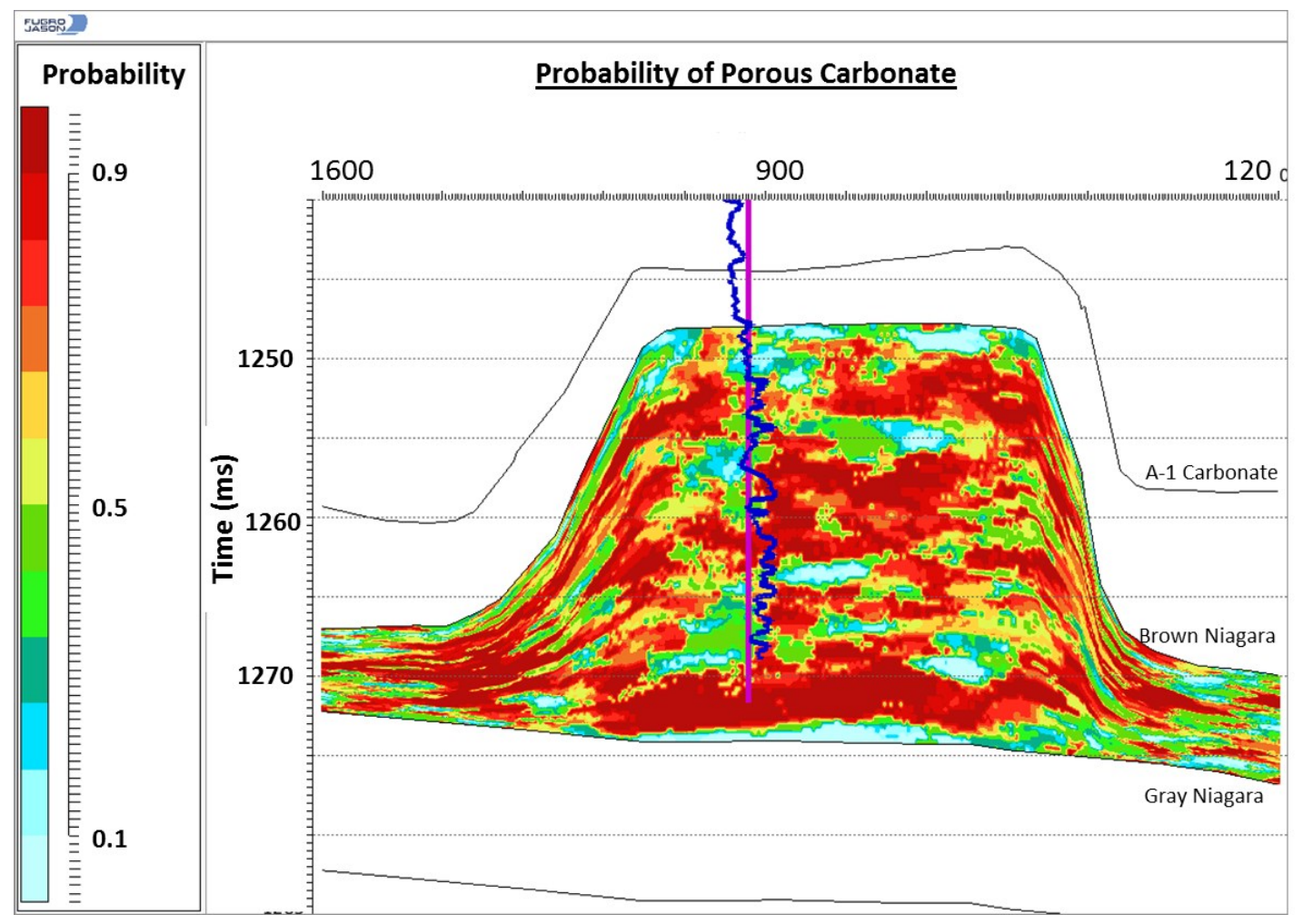

Figure 6.16: The variability between ten realizations was used to determine probabilities for porous carbonate. Neutron porosity Well log is shown in blue. The Springdale well used to drive the statistics for these results with a cutoff of porous $=$ $N P H I>0.07$. 

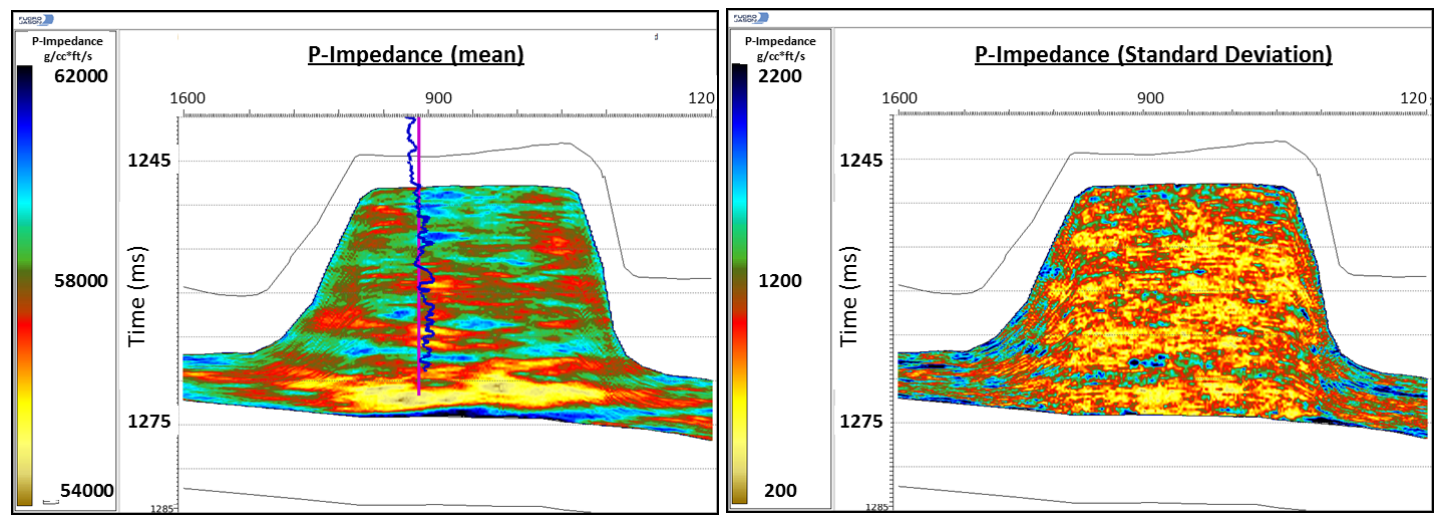

Figure 17: The results of mean P-Impedance combined from ten realizations and its standard deviation.
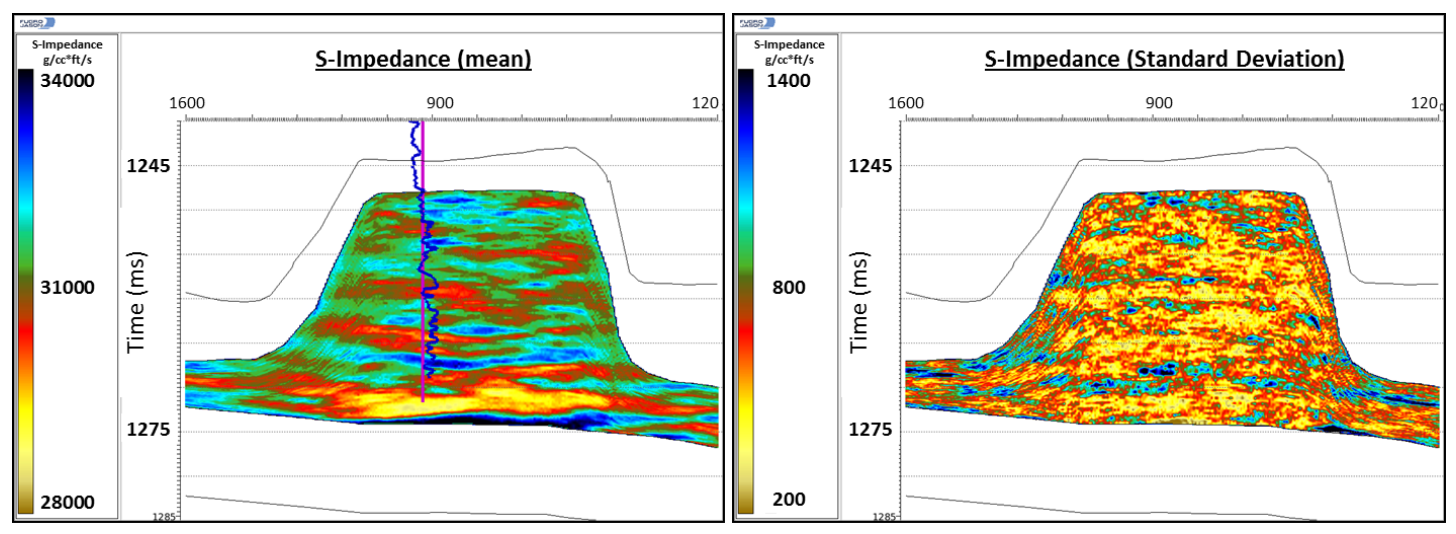

Figure 6.18: The results of mean S-Impedance combined from ten realizations and its standard deviation.
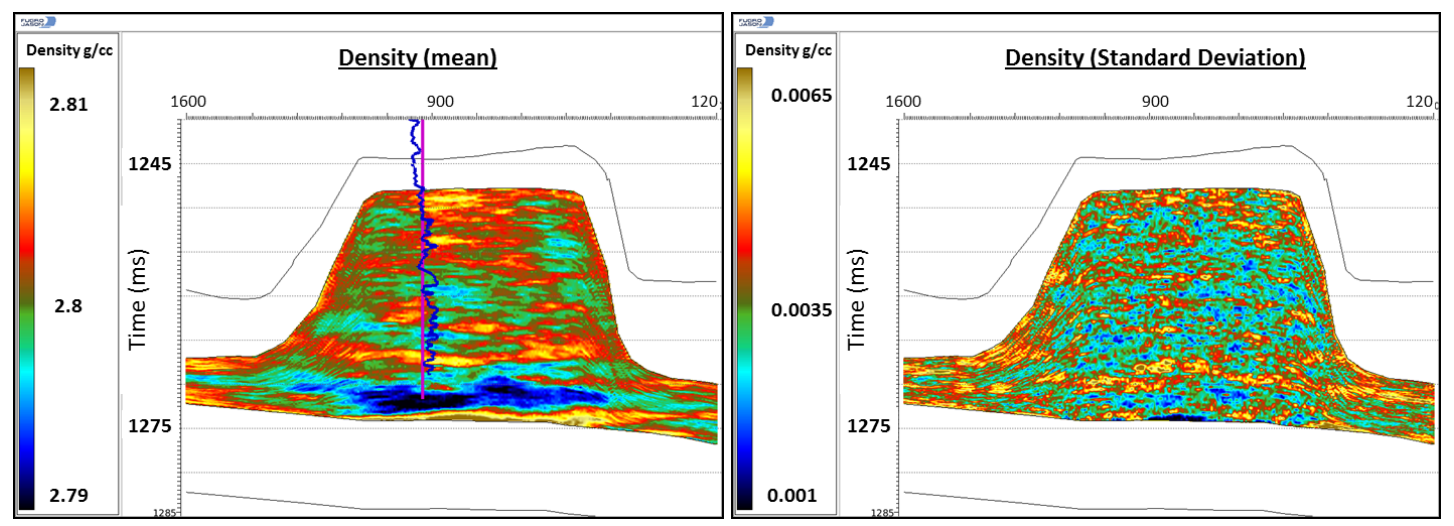

Figure 6.19: The results of mean Density combined from ten realizations and its standard deviation. 


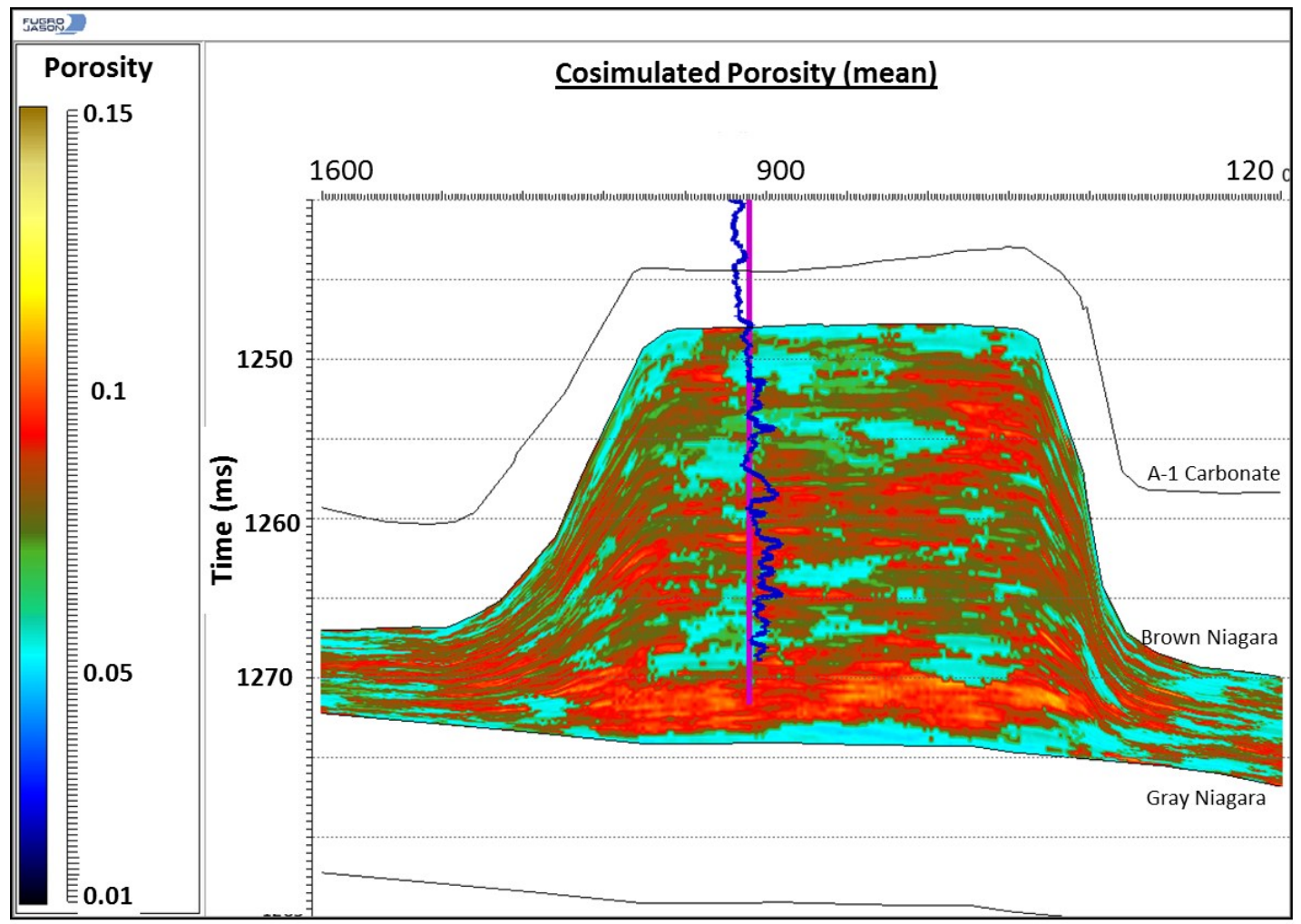

Figure 6.20: The neutron porosity well log is overlaid the cosimulated porosity results at Springdale reef.

\subsection{Conclusion}

Seismic inversion either; post-stack or pre-stack, deterministic or geostatistical become a standard part of the workflow for quantitative reservoir characterization. Elastic properties and petrophysical parameters from wells can be calibrated through the petro-elastic model; hence, the reservoir properties can be estimated at any point within the survey grid.

The deterministic simultaneous AVO inversion over Coldspring showed the porosity distribution inside the reef; areas of high porosity (low impedance zones) and the areas of low porosity (high impedance zones). Because the impedance is a layer property, the inversion results were used to pick the OOWC horizon that separates high and low porosity zones. The reefs are poorly suited for direct detection of hydrocarbons because the limestone and dolomite comprising the formation are highly incompressible and their velocities are very weakly dependent on fluid content. The change in impedances noticed in the inversion results over Coldspring is 
due to the lithology change not a fluid indicator, although it was indeed coincident with the original oil-water contact.

Given that deterministic inversion is much more quickly performed than geostatistical inversion, running the deterministic inversion prior to the geostatistical inversion will minimize some of uncertainty in building the geological model needed for geostatistical inversion. The deterministic results at Coldspring showed that the Brown Niagara layer (reef) is divided into two major zones of porosity and this suggested a two layer case model for geostatistical inversion. Such a model avoided a probability density function with bimodal distribution.

Deterministic inversion ignores uncertainty whereas geostatistical inversion is designed to explicitly account for uncertainty. The geostatistical method provides a set of solutions, of lithology and elastic properties that modeled jointly. The MCMC samples these pdfs to find a fair set of possible outcomes, each consistent with the input seismic. From the variability of these outcomes, uncertainties and probabilities can be inferred.

Geostatistical inversion result over Coldspring offered and a higher detailed image for the reef. The variability between ten realizations was used to determine probabilities for porous carbonate. The standard deviation around the mean of elastic properties was used for quality control the results and for uncertainty assessment. The results of standard deviation confirm that the spatial range of uncertainty is consistent with the estimated lateral extent of carbonate units.

Of reservoir engineering properties (porosity, permeability and SW), porosity is the most tightly coupled to P-impedance attribute. The porosity distribution is often the key to effective heterogeneity of carbonate reservoirs. Such an evaluation would improve the reservoir characterization and provide a number of reservoir development options. The lithology probability volume and the elastic properties output from geostatistical inversion are interdependent and provide a more accurate porosity through the cosimulation workflow.

Springdale and Coldspring reefs belong the same trend in Michigan basin and some similarity in facies is expected between them. The possibility of using geostatisitics from a well-known reef or a nearby field and apply them in a reef with a limited or no well information is addressed in this article. We applied the exact same inputs of geostatistics used in Coldspring inversion on the Springdale reef. The two reefs are situated at two different depths so the trend was removed and the rest of workflow operated with the residuals. An external trend created using a scaled velocity field was added back to obtain the absolute elastic properties.

For the top of the reef at Springdale, the geostatistical results showed an agreement with previous deterministic results and the geology known for this reef. Also, the results showed the rest of the reef has value of porosity higher than what is known 
from previous observation. We attribute this to two points; first the geostatistics at Coldspring derived from the upper part of the reef (above the OOWC horizon) but used for the entire reef at Springdale. Secondly, the lithology type created for Coldspring was based on neutron porosity cutoff equal to $5 \%$ or greater which happened to fit the porosity distribution for this reef above and below OOWC horizon. This 5\% cutoff is not particularly insightful at Springdale.

A regular geostatisical workflow was performed using a well penetrating the reef with synthesized shear sonic and density logs. The lithology probability volume of multiple realizations obtained. The results provide insight into the internal geometry of this reef with higher details compared to the deterministic inversion results, not discussed in this article. Barriers of tight carbonate are evident and the image matches the geologic interpretation of the reef model quite well. Because of the data covered angle range of $25^{\circ}-72^{\circ}$ using 6 partial stacks with above and beneath approach (Ibrahim et al., 2012), a reasonable density result was obtained. The cosimulated porosity of high details will be of great benefit to reservoir management over this field.

\subsection{References}

Caers J., 2005, Petroleum Geostatistics, Society of Petroleum Engineers, 88 pages.

Carrillo P., Milagrosa A., Bryans B., and Turpening R., 2007, Attenuation coefficient tomogram and $\mathrm{Q}$ distribution image from crosswell survey in the Northern Reef Trend of Michigan Basin; SEG Expanded Abstracts 26, 1252. DOI:10.1190/1.2792731

Caughlin, W.G., F.J. Lucia, N.L. McIver, (1976), The Detection and Development of Silurian Reefs in Northern Michigan, Geophysics, Vol. 41, No. 4

Connolly P., 1999, Elastic impedance: The Leading Edge, VOL. 18, NO. 4, P. 438 452.

Connolly, P. and Kemper, M., 2007, Statistical uncertainty of seismic net pay estimations. The Leading Edge, October 2007, 1284-1289.

Contreras A., Verdin C. T. and Fasnacht T., 2006, AVA simultaneous inversion of partially stacked seismic amplitude data for the spatial delineation of lithology and fluid units of deepwater hydrocarbon reservoirs in the centeral Gulf of Mexico: Geophysics, VOL 71, NO. 4, P. E41-E48.

Cumings, E.R. (1932), Reefs or Bioherms? Geol. Soc. America Bull., Vol. 43, No. 1 
Debeye, H.W. J., and P. van Riel, 1990, Lp-norm deconvolution: Geophysical Prospecting, 38, 381-404.

Deutsch C.V., 2002, Geostatistical Reservoir Modeling, Oxford University Press, 376 pages.

Droste, J.B., R.H. Shaver, (1985), Comparative Stratigraphic Framework for Silurian Reefs-Michigan Basin to Surrounding Platforms, in Special Paper \#4 Ordovician and Silurian Rocks of the Michigan Basin and its Margins, Mich. Basin Geo. Soc., Lansing, Mich.

Dubrule, O., 2003, Geostatistics for seismic data integration in earth model. Distinguished Instructor, Series, No. 6.

Gill, D., 1973, Stratigraphy, facies, evolution and diagenesis of productive Niagaran Guelph reefs and Cayugan sabkha deposits, the Belle River Mills gas field, Michigan basin: Ph.D. Dissertation, University of Michigan, Ann Arbor, 275 p.

Gill, D., 1977, The Belle River Mills gas field: Productive Niagaran reefs encased by sabkha deposits, Michigan basin: Michigan Basin Geological Society Special Paper 2, $188 \mathrm{p}$.

Hill, S., 2005, Inversion-based thickness determination: The Leading Edge, 24, 477480.

Huh, J.M.(1973), Geology and Diagenesis of the Niagaran Pinnacle Reefs in the Northern Shelf of the Michigan Basin, Ph.D. thesis, Univ. of Michigan, Ann Arbor, Michigan

Ibrahim, M. S, Pennington, W. D. and Turpening, R. M, 2009, Crosswell seismic imaging and inversion of a Michigan reef from above and from beneath: SEG Expanded Abstracts 28, 3934-3938

Ibrahim, M. S, Pennington, W. D. and Turpening, R. M, 2010, Crosswell seismic imaging of acoustic and shear impedance in Michigan reef: The Leading Edge, June, vol. 29 , no. 6 , p. $706-711$

Jarvis, K., Folkers, A. and Saussus, D., 2007: Reservoir compartment prediction of the Simpson field from geostatistical inversion of AVO seismic data: ASEG Extended Abstract 2007, Perth, Western Australia, p. 1-3

Jodry, R. L., 1969, Growth and dolomitization of Silurian reefs, St. Clair County, Michigan: Bulletin of the American Association of Petroleum Geologists, Vol 53, No., 4, April, 1969, PP. 957-981 
Latimer, R. B., Davison, R. and P. van Riel, 2000, An interpreter's guide to understanding and working with seismic-derived acoustic impedance data: The Leading Edge, 19, 242-256.

Lazaratos. S. K., 1993, Cross-well reflection imaging: Ph.D. Thesis, Stanford University.

Lazaratos, S. K., Rector, J. W., Harris, J. M., 1991, High resolution cross-well reflection imaging: 61st Annual International Meeting, SEG, Expanded Abstracts, 350-353.

Lowenstam, H.A., (1950), Niagaran Reefs of the Great Lakes Area, Jour. of Geology, Vol. 58

Lu, S. and McMechan, G, 2004, Elastic impedance inversion of multichannel seismic data from unconsolidated sediments containing gas hydrate and free gas: Geophysics, Vol. 69, No. 1 (January-February 2004); P. 164-179,

Mesolella, K., J., Robinson, J.D., Mccormick, L.M. and Ormiston, A.R., 1974, Cyclic deposition of Silurian carbonates and evaporates in Michigan basin: Bulletin of the American Association of Petroleum Geologists, Vol 58, No., 1, January, 1974, PP. $34-62$

Nelson, H.F., C.W. Brown, J.H. Brineman (1962), Skeletal Limestone Classification in Classification of Carbonate Rocks, Amer. Assoc. Petrol. Geol., Mem. 1

Pendrel John, 2006, Seismic inversion - A critical tool in reservoir characterization: Scandinavian Oil-Gas Magazine, NO. 5/6.

--------, 2007, Advanced techniques for simultaneous AVO inversion (RockTrace, RockMod): CSPG CSEG Convention, p. 115-116

Pendrel, J., and Van Riel P., 1997, Methodology for seismic inversion and modeling: A western Canadian reef example: CSEG Recorder, 22, 5-15.

Pendrel, J., Debeye, H., Pederson, T. R., Goodway, B., Dufour, J., Bogaards, M., Stewart, R. 2000, Estimation and interpretation of $\mathrm{P}$ and $\mathrm{S}$ impedance volumes from simultaneous inversion of P-wave offset data, CSEG Ann. Mtg. Abs. paper AVO 25.

Pennington W. D., Ibrahim, M., Turpening, R.M., Trisch, S.P., Richardson, J.P., Asiala, C., and Mabrouk, W. (2008), Crosswell Seismic Amplitude-Versus-Offset for Detailed Imaging of Facies and Fluid Distribution within Carbonate Oil Reservoirs, report to Dept of Energy, available at www.osti.gov/servlets/purl/946424-Mm7X2f/. 
Pray, L.C. (1958), Fenestrate Bryozoan Core Facies, Mississippian Bioherms southwest United States, Jour. Sed. Petrology, Vol. 28

Rector J.W., Lazaratos S. K., Harris J. M. and Schaacks M.V., 1995, High-resolution crosswell imaging of a west Texas carbonate reservoir: Part 3-Wavefield separation of reflections: Geophysics, 60, P.692-701

Sams, M. and Saussus, D., 2007, Estimating uncertainty in reserves from deterministic inversion. Petroleum Geostatistics 2007 - Cascais, Portugal, Extended Abstracts, P17.

Sams, M. and Saussus, D. (2008) Comparison of uncertainty estimates from deterministic and geostatistical inversion. SEG Technical Program Expanded Abstracts 2008: pp. 1486-1490.

Sengupta, M. and Bachrach, R., 2007, Uncertainty in seismic-based pay volume estimation: Analysis using rock physics and Bayesian statistics. The Leading Edge, February 2007, 184-189.

Shafer, R.H. (1974), Silurian Reefs of Northern Indiana, Reef and Interreef Macrofaunas, Bull. Am. Assoc. of Petrol. Geol. Vol. 58, No. 6

Sharma, G. D., (1966), Geology of Peters Reef, St. Clair County, Michigan, Bull. Am. Assoc. of Petrol. Geol. Vol. 50, No. 2

Sheriff, R. E., Geldart, L. P., (1995), 2nd Edition. Exploration Seismology. Cambridge University Press.

Smith, B.A., Gallagher, J.G.,Hoover, G.M., and Hufford, J.M.,1993, Characteristics of crosswell wave propagation at varying well offsets, 55th mtg.: Eur. Assn. of Expl. Geophys., Session: C007

Tarantola Albert, 1984, Inversion of seismic reflection data in acoustic approximation: Geophysics, VOL. 49, NO. 8, P. 1259-1266.

------, 1986, A strategy for nonlinear elastic inversion of seismic reflection data: Geophysics, VOL. 51, NO. 10, P. 1893-1903.

Trisch, S.P. 2006, Crosswell Seismic Amplitude Variation with Angle Studies at a Niagaran Reef, MS Thesis, Michigan Technological University.

Wyllie, A.S., and Wood, J.R., 2005, Well-log tomography and 3-D imaging of core and log-curve amplitudes in a Niagaran reef, Belle River Mills field, St. Clair County, Michigan, United States, Bull. Amer. Assoc. Petroleum Geologists, 89, 409-433. 


\section{Overall Conclusions}

Crosswell seismic imaging has demonstrated its ability to provide extremely highquality images of reservoirs such that internal features within the reef can be imaged and characterized. Resolution is about 40 times that of surface seismic in the same area $(50 \mathrm{~Hz}$ versus $2000 \mathrm{~Hz})$.

The crosswell seismic survey can be adjusted to have a unique geometry that provides images made reflections from beneath and using reflections from above the various reflecting horizons. The wide angles used in crosswell imaging result in amplitudeversus-angle (AVA) features not usually observed in surface data. These features include reflections from angles that are near critical and beyond critical for many of the interfaces; some of these reflections are visible only for a small range of angles, presumably near their critical angle. Combining the results from images obtained from above with those from beneath provides additional information, first by exhibiting ranges of angles that are different for the two images, especially for reflectors at shallow depths, and second, by providing additional constraints on the solutions for Zoeppritz equations.

The incident of angles recorded on any reflector is highly dependent on the accuracy of velocity model as well as the shape of the reflector. The complex shape, as reef flanks, would make the angle range recorded is very wide that would impossible for any processing workflow to image it. The dataset from beneath may provide the only way to map based the depth of the source and receivers pair below the target. Still the difficulty of mapping dipping reflectors and the migration issues may be a challenge in such case. Also, another benefit of having the dataset from beneath that it may provide extra information by having range of angles that does not exist in the above dataset, especially for reflectors at shallow depths where getting the same angle range may not be possible due to a shortage of distance to the surface.

The interpretation of Crosswell image is difficult due to the stretching distortion introduced by the current mapping algorithm, so comparing the amplitude-versusangle (AVA) gathers of actual crosswell data with exact solution of Zoeppritz equations will provide a better understanding for the crosswell data itself and will help in most detailed AVA studies.

One advantage of combining the AVA analysis with inversion is you better understand where the inversion result you trust most or where the increase in amplitudes is because the near critical angle not because strong contrast in elastic properties. 
The deterministic inversion of seismic amplitude data algorithm can be used in two ways; full-stack (which is often assumed for surface-collected data to be zero-offset "acoustic"), or based on angle-stacks representing limited ranges of angles in each stack (non-zero offset, or "elastic"). Particularly in our crosswell data, the full-stack inversion is, of course, not "acoustic" because of the very-wide angles involved and also because of geometry of the crosswell survey no zero-offset can be recorded. Although "acoustic" inversion also provides meaningful results, the interpreter must recognize that the resulting image is not truly "acoustic" impedance, but some sort of narrow-angle "elastic" impedance, referred to in this work as "apparent" impedance.

The packaged inversion procedures assume that the reflection series is encountered from the top down, so until techniques are developed that properly account for crosswell seismic inversion from beneath, we treat the two (above and beneath) data sets using two approaches based on the layers contrast of elastic properties.

Our approach in case of strong contrast (e.g Salt over Carbonates), the reflection observed from above is not just the inverse of the reflection observed from below (with a simple sign change), at any angles other than normal incidence is treating the two datasets independently, rather than providing additional constraints on the simultaneous inversion. By "flipping" the "from-beneath" seismic traces (top-down, or last-sample to first-sample), then perform the inversions using standard software, and then "flip" the resulting image back so that the shallow depths are again on top.

For any interface, the results AVA characters must result from identical contrasts in elastic properties in the two sets of images, albeit in reverse order. In case of small contrast, we chose to combine the small-angle gathers from above with the largeangle gathers from beneath by "flipping" the seismic traces of both data sets; from above and from beneath so that the inversion can take place from beneath. The flipping process or time-reversed traces is flipping the trace top-down, or last-sample to first-sample. Then, we sign-reversed the data (gathers) from above, so that they are (nearly) equivalent to the appropriate gathers would have been from beneath. Then perform the inversions using standard software, and then time-reverse the resulting image back (flip) so that the shallow depths are again on top. This approach will honor the sign of the small-angle gathers after switching the time order (as if they were being reflected from beneath, which is valid for small angles - below $50^{\circ}$ ), but it will also honor the Zoeprittz solutions for the large-angle gathers, since it will be correct for from beneath inversion. This combined approach taking advantage of the better signal in both ranges, and extending the overall angle range available for inversion.

While the deterministic inversion is designed to ignore uncertainty and yields only a single model of elastic properties, the geostatistical inversion produces multiple models (realizations) of elastic properties, lithology and reservoir properties. These realizations combined to yield lithology probability volumes and also can be used 
directly to quantify the uncertainty in any of the resulting model properties. The geostatistical inversion outputs of greater detail compared to what we can be obtained using seismic alone through deterministic inversion.

In case of analogues reservoirs, the possibility of using geostatistics from a wellknown reef or a nearby field and apply it in a reef with a limited or no well information is possible. A preconception and extra data resources should be used to control and constraint the geostatistical results.

Of reservoir engineering properties (porosity, permeability and SW), porosity is the most tightly coupled to P-impedance attribute. Determine the porosity distribution is key to evaluate the heterogeneity of the carbonate reservoirs. Such an evaluation would improve the reservoir characterization and perform a number of reservoir development options Both lithology probability volume as well as the elastic properties output from geostatistical inversion are dependent and give more freedom to obtain a better and accurate porosity through the cosimulation workflow. 


\section{References}

Caers J., 2005, Petroleum Geostatistics, Society of Petroleum Engineers, 88 pages.

Carrillo P., Milagrosa A., Bryans B., and Turpening R., 2007, Attenuation coefficient tomogram and Q distribution image from crosswell survey in the Northern Reef Trend of Michigan Basin; SEG Expanded Abstracts 26, 1252. DOI:10.1190/1.2792731

Caughlin, W.G., F.J. Lucia, N.L. McIver, (1976), The Detection and Development of Silurian Reefs in Northern Michigan, Geophysics, Vol. 41, No. 4

Connolly P., 1999, Elastic impedance: The Leading Edge, VOL. 18, NO. 4, P. 438 452.

Connolly, P. and Kemper, M., 2007, Statistical uncertainty of seismic net pay estimations. The Leading Edge, October 2007, 1284-1289.

Contreras A., Verdin C. T. and Fasnacht T., 2006, AVA simultaneous inversion of partially stacked seismic amplitude data for the spatial delineation of lithology and fluid units of deepwater hydrocarbon reservoirs in the centeral Gulf of Mexico: Geophysics, VOL 71, NO. 4, P. E41-E48.

Cumings, E.R. (1932), Reefs or Bioherms? Geol. Soc. America Bull., Vol. 43, No. 1

Debeye, H.W. J., and P. van Riel, 1990, Lp-norm deconvolution: Geophysical Prospecting, 38, 381-404.

Deutsch C.V., 2002, Geostatistical Reservoir Modeling, Oxford University Press, 376 pages.

Droste, J.B., R.H. Shaver, (1985), Comparative Stratigraphic Framework for Silurian ReefsMichigan Basin to Surrounding Platforms, in Special Paper \#4 Ordovician and Silurian Rocks of the Michigan Basin and its Margins, Mich. Basin Geo. Soc., Lansing, Mich.

Dubrule, O., 2003, Geostatistics for seismic data integration in earth model. Distinguished Instructor, Series, No. 6.

Gill, D., 1973, Stratigraphy, facies, evolution and diagenesis of productive Niagaran Guelph reefs and Cayugan sabkha deposits, the Belle River Mills gas field, Michigan basin: Ph.D. Dissertation, University of Michigan, Ann Arbor, 275 p. 
Gill, D., 1977, The Belle River Mills gas field: Productive Niagaran reefs encased by sabkha deposits, Michigan basin: Michigan Basin Geological Society Special Paper 2, $188 \mathrm{p}$.

Hill, S., 2005, Inversion-based thickness determination: The Leading Edge, 24, 477480.

Huh, J.M.(1973), Geology and Diagenesis of the Niagaran Pinnacle Reefs in the Northern Shelf of the Michigan Basin, Ph.D. thesis, Univ. of Michigan, Ann Arbor, Michigan

Ibrahim, M. S, Pennington, W. D. and Turpening, R. M, 2009, Crosswell seismic imaging and inversion of a Michigan reef from above and from beneath: SEG Expanded Abstracts 28, 3934-3938

Ibrahim, M. S, Pennington, W. D. and Turpening, R. M, 2010, Crosswell seismic imaging of acoustic and shear impedance in Michigan reef: The Leading Edge, June, vol. 29, no. 6, p. 706-711

Jarvis, K., Folkers, A. and Saussus, D., 2007: Reservoir compartment prediction of the Simpson field from geostatistical inversion of AVO seismic data: ASEG Extended Abstract 2007, Perth, Western Australia, p. 1-3

Jodry, R. L., 1969, Growth and dolomitization of Silurian reefs, St. Clair County, Michigan: Bulletin of the American Association of Petroleum Geologists, Vol 53, No., 4, April, 1969, PP. 957-981

Latimer, R. B., Davison, R. and P. van Riel, 2000, An interpreter's guide to understanding and working with seismic-derived acoustic impedance data: The Leading Edge, 19, 242-256.

Lazaratos. S. K., 1993, Cross-well reflection imaging: Ph.D. Thesis, Stanford University.

Lazaratos, S. K., Rector, J. W., Harris, J. M., 1991, High resolution cross-well reflection imaging: 61st Annual International Meeting, SEG, Expanded Abstracts, 350-353.

Lowenstam, H.A., (1950), Niagaran Reefs of the Great Lakes Area, Jour. of Geology, Vol. 58

Lu, S. and McMechan, G, 2004, Elastic impedance inversion of multichannel seismic data from unconsolidated sediments containing gas hydrate and free gas: Geophysics, Vol. 69, No. 1 (January-February 2004); P. 164-179, 
Mesolella, K., J., Robinson, J.D., Mccormick, L.M. and Ormiston, A.R., 1974, Cyclic deposition of Silurian carbonates and evaporates in Michigan basin: Bulletin of the American Association of Petroleum Geologists, Vol 58, No., 1, January, 1974, PP. $34-62$

Nelson, H.F., C.W. Brown, J.H. Brineman (1962), Skeletal Limestone Classification in Classification of Carbonate Rocks, Amer. Assoc. Petrol. Geol., Mem. 1

Pendrel John, 2006, Seismic inversion - A critical tool in reservoir characterization: Scandinavian Oil-Gas Magazine, NO. 5/6.

--------, 2007, Advanced techniques for simultaneous AVO inversion (RockTrace, RockMod): CSPG CSEG Convention, p. 115-116

Pendrel, J., and Van Riel P., 1997, Methodology for seismic inversion and modeling: A western Canadian reef example: CSEG Recorder, 22, 5-15.

Pendrel, J., Debeye, H., Pederson, T. R., Goodway, B., Dufour, J., Bogaards, M., Stewart, R. 2000, Estimation and interpretation of P and S impedance volumes from simultaneous inversion of P-wave offset data, CSEG Ann. Mtg. Abs. paper AVO 25.

Pennington W. D., Ibrahim, M., Turpening, R.M., Trisch, S.P., Richardson, J.P., Asiala, C., and Mabrouk, W. (2008), Crosswell Seismic Amplitude-Versus-Offset for Detailed Imaging of Facies and Fluid Distribution within Carbonate Oil Reservoirs, report to Dept of Energy, available at www.osti.gov/servlets/purl/946424-Mm7X2f/.

Pray, L.C. (1958), Fenestrate Bryozoan Core Facies, Mississippian Bioherms southwest United States, Jour. Sed. Petrology, Vol. 28

Rector J.W., Lazaratos S. K., Harris J. M. and Schaacks M.V., 1995, High-resolution crosswell imaging of a west Texas carbonate reservoir: Part 3-Wavefield separation of reflections: Geophysics, 60, P.692-701

Sams, M. and Saussus, D., 2007, Estimating uncertainty in reserves from deterministic inversion. Petroleum Geostatistics 2007 - Cascais, Portugal, Extended Abstracts, P17.

Sams, M. and Saussus, D. (2008) Comparison of uncertainty estimates from deterministic and geostatistical inversion. SEG Technical Program Expanded Abstracts 2008: pp. 1486-1490.

Sengupta, M. and Bachrach, R., 2007, Uncertainty in seismic-based pay volume estimation: Analysis using rock physics and Bayesian statistics. The Leading Edge, February 2007, 184-189. 
Shafer, R.H. (1974), Silurian Reefs of Northern Indiana, Reef and Interreef Macrofaunas, Bull. Am. Assoc. of Petrol. Geol. Vol. 58, No. 6

Sharma, G. D., (1966), Geology of Peters Reef, St. Clair County, Michigan, Bull. Am. Assoc. of Petrol. Geol. Vol. 50, No. 2

Sheriff, R. E., Geldart, L. P., (1995), 2nd Edition. Exploration Seismology. Cambridge University Press.

Smith, B.A., Gallagher, J.G.,Hoover, G.M., and Hufford, J.M.,1993, Characteristics of crosswell wave propagation at varying well offsets, $55^{\text {th }} \mathrm{mtg}$.: Eur. Assn. of Expl. Geophys., Session: C007

Tarantola Albert, 1984, Inversion of seismic reflection data in acoustic approximation: Geophysics, VOL. 49, NO. 8, P. 1259-1266.

------, 1986, A strategy for nonlinear elastic inversion of seismic reflection data: Geophysics, VOL. 51, NO. 10, P. 1893-1903.

Trisch, S.P. 2006, Crosswell Seismic Amplitude Variation with Angle Studies at a Niagaran Reef, MS Thesis, Michigan Technological University.

Wyllie, A.S., and Wood, J.R., 2005, Well-log tomography and 3-D imaging of core and log-curve amplitudes in a Niagaran reef, Belle River Mills field, St. Clair County, Michigan, United States, Bull. Amer. Assoc. Petroleum Geologists, 89, 409-433. 